Turbulent exchange of energy, momentum, and reactive gases between high vegetation and the atmospheric boundary layer

M. M. Shapkalijevski 


\section{Thesis committee}

\section{Promotors}

Prof. Dr M.C. Krol

Professor of Air Quality and Atmospheric Chemistry

Wageningen University

Prof. Dr J. Vilà-Guerau de Arellano

Personal chair, Meteorology and Air Quality Group

Wageningen University

\section{Co-promotors}

Dr A.F. Moene

Associate professor, Meteorology and Air Quality Group

Wageningen University

Dr H.G. Ouwersloot

Postdoctoral researcher in Atmospheric Dynamics and Chemistry

Max-Planck Institute for Chemistry, Mainz, Germany

\section{Other members}

Prof. Dr R. Uijlenhoet, Wageningen University

Prof. Dr T. Foken, University of Bayreuth, Germany

Prof. Dr J. Lelieveld, Max-Planck Institute for Chemistry, Mainz, Germany

Dr F. Bosfeld, The Royal Netherlands Meteorological Institute (KNMI), de Bilt

This research was conducted under the auspices of the Graduate School for Socio-Economic and Natural Sciences of the Environment (SENSE). 


\title{
Turbulent exchange of energy, momentum, and reactive gases between high vegetation and the atmospheric boundary layer
}

\author{
M. M. Shapkalijevski
}

Thesis

submitted in fulfilment of the requirements for the degree of doctor at Wageningen University

by the authority of the Rector Magnificus

Prof. Dr A.P.J. Mol

in the presence of the

Thesis Committee appointed by the Academic Board

to be defended in public

on Monday 1 May 2017

at 4 p.m. in the Aula. 
M.M. Shapkalijevski

Turbulent exchange of energy, momentum, and reactive gases between high vegetation and the atmospheric boundary layer, 170 pages.

$\mathrm{PhD}$ thesis, Wageningen University, Wageningen, NL (2017)

With references, with summary in English

ISBN: 978-94-6343-084-5

DOI: $10.18174 / 404603$ 


\section{Contents}

1 Introduction $\quad 9$

1.1 Tall plant canopies in the Earth's system . . . . . . . . . . . . . . . . . . 9

1.2 The roughness sublayer within the land-PBL system . . . . . . . . . . . . . . 13

1.3 Observed and modelled turbulent fluxes in and above canopies . . . . . . . . . 17

1.3.1 Measuring turbulent fluxes: CHATS a comprehensive experiment . . . . 17

1.3.2 Parameterizing turbulent fluxes . . . . . . . . . . . . . 18

1.4 Research questions, strategy and outline . . . . . . . . . . . . . . . . 20

2 Influence of canopy seasonal changes on turbulence parameterization within the roughness sublayer over an orchard canopy $\quad 23$

2.1 Introduction . . . . . . . . . . . . . . . . . . . 24

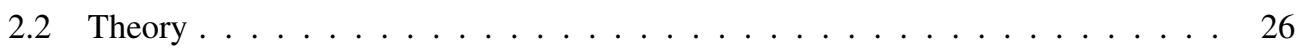

2.2.1 Parameterization of $K$ based on dimensionless gradients . . . . . . . . . 26

Modification within the RSL . . . . . . . . . . . . 27

Displacement height . . . . . . . . . . . . . . . 28

2.2.2 Parameterization of $K$ based on TKE and its dissipation . . . . . . . . . 29

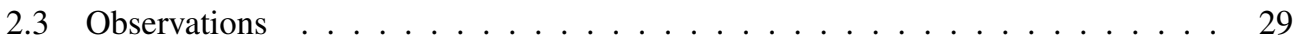

2.3.1 CHATS experiment: Site and instrumentation . . . . . . . . . . . . 29

2.3 .2 Data processing . . . . . . . . . . . . . . . 30

2.4 Results and discussion . . . . . . . . . . . . . . . . . . 32

2.4.1 Phenology effects on the observed turbulent exchange coefficients . . . . 32

2.4.2 Phenology effects on $K$-parameterization . . . . . . . . . . . . . . . . 34

The role of the displacement height . . . . . . . . . . . . 34

The role of foliage on $K$-parameterization using dimensionless gradients ........................ 36

Traditional MOST . . . . . . . . . . . . . . 36

RSL-corrected MOST . . . . . . . . . . . . . . . 38

The role of phenology on $K$-parameterization based on TKE and dissipation .................. . . 42 
2.5 Conclusions . . . . . . . . . . . . . . . . . . . . 46

2A Appendix: Analysis of the vertical flux divergence . . . . . . . . . . 48

2B Appendix: Steps to calculate TKE dissipation from the CHATS data . . . . . . 48

3 Integrating canopy and large-scale effects in the convective boundary-layer dynamics during CHATS experiment 51

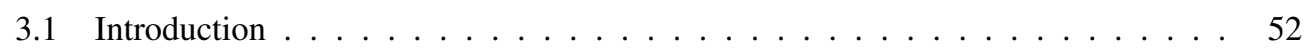

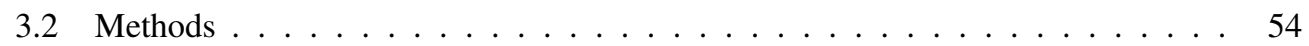

3.2 .1 CHATS data . . . . . . . . . . . . . . . . 54

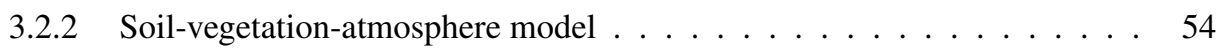

3.2 .3 Research strategy . . . . . . . . . . . . . . . . . . . . . 57

3.3 Model validation . . . . . . . . . . . . . . . . . . . . 59

3.3.1 Radiation and surface energy balance . . . . . . . . . . . . . . . 59

3.3 .2 CBL dynamics . . . . . . . . . . . . . . . . . . . 62

3.4 The wind in the RSL and effects on bulk momentum budget . . . . . . . . . 65

3.5 Heat and moisture . . . . . . . . . . . . . . . . . . . . 68

3.6 Discussion . . . . . . . . . . . . . . . . . . . . . . . . . . . 69

3.7 Conclusions . . . . . . . . . . . . . . . . . . . . 72

3A Appendix: Mixed-layer model initial and boundary conditions . . . . . . . . 74

3B Appendix: Momentum budget . . . . . . . . . . . . . . . 76

3C Appendix: Estimating the boundary-layer height from LIDAR data . . . . . . . 78

4 Combined effects of deposition, entrainment and chemistry on the ozone budget over an orchard canopy $\quad 83$

4.1 Introduction . . . . . . . . . . . . . . . . . . . . . . 84

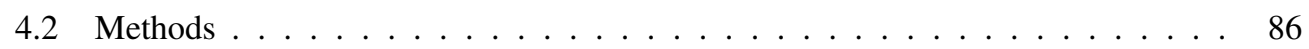

4.2 .1 Observations and data treatment $\ldots \ldots \ldots \ldots \ldots$

4.2 .2 Inferring gas fluxes $\ldots \ldots \ldots \ldots \ldots \ldots \ldots$

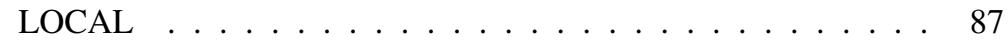

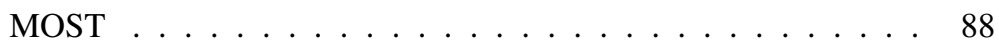

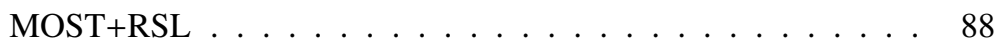

Inversed Localized Near-Field (ILNF) method . . . . . . . . . 89

4.3 Modelling the ozone budget $\ldots \ldots \ldots$. . . . . . . . . . . 90

4.3 .1 Model and case study . . . . . . . . . . . . . . . . . . . 90

4.3 .2 Entrainment. . . . . . . . . . . . . . . . . . . . . 91

4.3 .3 Dry deposition . . . . . . . . . . . . . . . . . . . 92

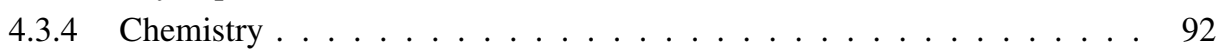

4.3 .5 Advection . . . . . . . . . . . . . . . . . . . . 94

4.4 Atmospheric chemistry during CHATS . . . . . . . . . . . . . . . . . 95 
4.4.1 General characterization . . . . . . . . . . . . . . . 95

4.4.2 Inferring fluxes of radicals from observed mean mixing ratios $\ldots \ldots 100$

4.4.3 Impact of irrigation on ozone exchange . . . . . . . . . . 102

4.5 Analysis of the diurnal ozone budget . . . . . . . . . . . . . . 105

4.5 .1 Initial and boundary conditions $\ldots \ldots \ldots \ldots \ldots$

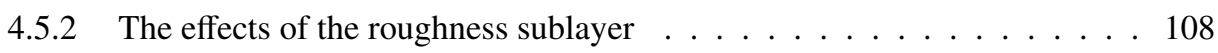

4.5.3 Sensitivity of simulated $\mathrm{O}_{3}$ and $\mathrm{NO}_{x} \ldots \ldots \ldots \ldots \ldots \ldots$

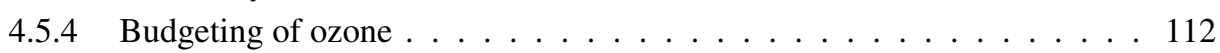

4.6 Conclusions . . . . . . . . . . . . . . . . . . . . . . . . . 114

4A Appendix: Heat flux and friction velocity above and within the canopy . . . . . 116

Above the canopy . . . . . . . . . . . . . . . 116

Within the canopy. . . . . . . . . . . . . . . . . . 117

4B Appendix: Parameterizing ozone fluxes above CHATS canopy . . . . . . . . 117

5 General discussion and future recommendations $\quad 121$

5.1 From dense to sparse canopies . . . . . . . . . . . . . . . . . 123

5.2 From near-neutral conditions to increased atmospheric (in)stability . . . . . . 128

5.3 From passive to active chemical transformation in and above canopies . . . . . 128

5.4 From isolated canopy to canopy coupled to larger-scale processes . . . . . . . 131

6 Summary 133

$\begin{array}{ll}\text { References } & 137\end{array}$

$\begin{array}{ll}\text { Acknowledgements } & 161\end{array}$

$\begin{array}{lr}\text { IMPRS certificate } & 165\end{array}$

$\begin{array}{lr}\text { SENSE certificate } & 168\end{array}$ 


\title{
1 \\ Introduction
}

\begin{abstract}
Atmospheric flow interacts with plant canopies. This thesis deals with the representation of the exchange of energy, momentum and chemical reactants between the land, covered by high vegetation, and the lowest part of the atmosphere, named as atmospheric boundary layer $(A B L)$, under daytime conditions. This chapter explains the relevance of studying the tall plant canopy-atmosphere interaction within the Earth system, and introduces the roughness sublayer (RSL), as the layer just above the canopy in which the atmospheric flow is directly affected by the presence of roughness elements. The chapter ends by summarizing the main methods, research questions and strategy used in this thesis.
\end{abstract}

\subsection{Tall plant canopies in the Earth's system}

The Earth's surface strongly influences and modulates the planetary boundary layer (PBL), a part of the atmosphere most directly affected by solar heating of the Earth's surface (Stull 1988). As such, it plays a crucial role in connecting surface properties with atmospheric conditions. This interaction is relevant in the vertical direction, governed by atmospheric turbulence (mixing), and in horizontal directions, mainly controlled by the surface heterogeneity. The earth-surface heterogeneity, defined by the mosaic of oceans, land, mountains, vegetation, are of importance for the large-scale variations in weather and climate (Betts et al. 1996; Pielke et al. 1998). Focusing on land, the variation of the surface types over the globe has an important impact on global 
(Claussen et al. 2004) and regional (e.g. Koster et al. 2004) climate. These surface properties largely contribute to the exchange of heat, water, momentum and chemical compounds between the land and atmosphere, strongly affecting the thermal, hydrological and aerodynamical characteristics of the PBL (Nicholson 1988) as well as its chemical composition (Karl and Trenberth 2003). For instance, during daytime water surfaces (e.g. oceans) are normally cooler than the land due to the high specific heat and thermal conductivity of water and high expenditure of energy for evaporation. For non-vegetated surface, the surface albedo, the fraction of solar energy reflected from the Earth back into space, and the surface temperature are directly dependent on the soil moisture content (Idso et al. 1975). In turn, vegetated surfaces influence the surface temperature and albedo due to the altered solar radiation interception within the canopies, which influence the reflection and emission of radiation (Dickinson 1983). These illustrations show that the land surface characteristics, quantified through thermal effects, surface (soil) evaporation and plant transpiration, play a crucial role in determining how solar energy is exchanged (transferred) back to the atmosphere. The exchange of incoming (to the Earth surface) and outgoing (from the Earth surface) radiation forms the radiative energy balance resulting in net radiation $(\mathrm{Rn})$. In terms of heat fluxes, this exchange is quantified by the surface energy balance (SEB) and is mainly composed by the following terms: surface temperature flux (sensible heat flux, $\mathrm{SH}$ ), surface moisture flux (latent heat flux, LE), the heat storage in the soil (ground heat flux, G) and the net radiation. Ideally, the sum of the first three terms results in net radiation $(\mathrm{Rn}=\mathrm{SH}+\mathrm{LE}+\mathrm{G})$. In reality however other terms, such as photosynthesis (an uptake of carbon dioxide, water and solar energy by the vegetation in the process of production nutrients), advection (horizontal transport of air quantities) or heat storage in plant biomass, can also be of specific relevance for the total SEB (Oliphant et al. 2004).

The exchange of energy, momentum and matter between the vegetated surface and the atmosphere depends on the surface roughness, which is characterized by the height, shape and density of the vegetation cover (Lettau 1969). The variation of these characteristics further influences the surface temperature and albedo, which additionally affect the SEB (Wilson et al. 1987; Dickinson; Henderson-Sellers 1988).

During this thesis, we define high vegetation as a canopy with average height larger than few meters. This mainly includes coniferous and deciduous forests, but also agricultural orchards and even corn canopies. Figure 1.1 shows the relevance of tall plant canopies, as they cover approximately $10 \%$ of the Earth's surface or around $31 \%$ of the total land. As such they influence climate locally and on large scales (Bonan 2008). Energy, water, carbon dioxide, and other compounds, including chemically reactive compounds, are continuously exchanged between the forests and the atmosphere (Gao et al. 1989). These exchange processes modify the physical properties of the PBL, as well as its composition, and occur at different scales locally, on the scale of an orchard (e.g. $\sim 1 \mathrm{~km} 2$ ), regionally, on the scale of the Amazon rainforest, and globally, as large biomes respond to environmental pressures associated with climate change. 


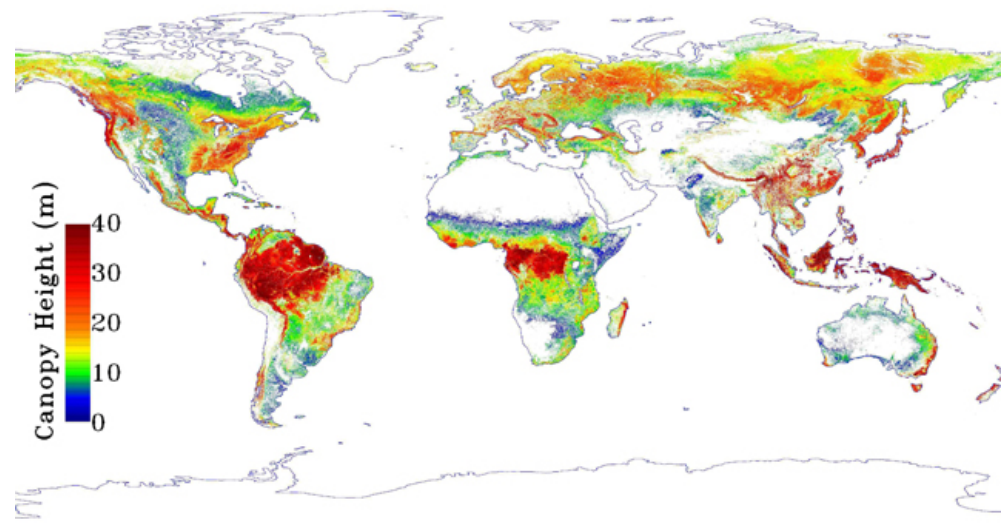

Figure 1.1: Global map of canopy height at 1-km spatial resolution, using 2005 data from the Geoscience Laser Altimeter System (GLAS) aboard ICESat (Ice, Cloud, and land Elevation Satellite) (source: Simard et al. 2011).

Due to its significance and the need of its representation, scientific methods were developed in order to investigate the interaction between high vegetation and the atmosphere and to quantify its effects on weather and climate. The commonly used scientific methods often combine direct measurements within and above canopies using eddy covariance (EC) flux towers (e.g. Baldocchi et al. 2001) and mathematical models of the soil-vegetation-atmosphere exchange processes with different complexity and spatial and temporal resolution (e.g. Bonan 2015). Recently, the use of remote satellite sensors in combination with local flux observations and atmospheric models is also an often used method.

$\mathrm{EC}$ is a technique to directly measure turbulent fluxes by correlating vertical wind velocity fluctuations with the deviation of the gas concentrations, temperature, humidity, or even horizontal components of the wind velocity. This method requires state-of-the-art fast response instruments with high precision, since the turbulent flow is characterize by a wide range of spatial and temporal scales that range from large-scale eddies (hundreds of meters and hour(s)) to Kolmogorov scales (millimeters and seconds) (Kolmogorov 1941). Therefore, the EC methods are the most explicit method to observe turbulent fluxes, and they are rapidly developing their scope, standards and applicability (Foken 2008b; Moene and Van Dam 2014; Monson and Baldocchi 2014).

With respect to modelling technique, we need to distinguish two techniques: the simulating techniques that treat turbulence explicitly (resolving) and the models that parameterize turbulence. The state-of-the-art scientific tools to simulate the complex turbulent exchange processes within the PBL is the Large Eddy Simulation (LES). LES is a numerical tool capable to simulate the atmospheric turbulent flow by resolving all the larger scales of turbulent motions as they evolve in 
space and time and parameterizing only the smallest ones in three dimensional domain (Wyngaard et al. 1984; Pope 2000; Wyngaard 2010). As computers are becoming stronger to perform longer simulations and larger domains with high resolution in present days, the LES are more often used to study the land-atmosphere (Patton et al. 2005) and canopy-atmosphere (Finnigan et al. 2009; Patton et al. 2016) turbulent exchange on a process base. In turn, LES results are becoming a key complement to direct EC measurements. The modelling techniques in which the turbulence is parameterized are mainly based on the so called ' $K$-theory', based on which the fluxes of investigated quantities are related to the mean gradients of those quantities by turbulent exchange coefficient $K$ (see section 1.3.2 for more details).

In applying these methods, we are constantly improving our understanding on high vegetation, atmosphere, and climate interaction (Bonan 2008). For instance, climate impacts ecosystem (forests) functioning through global warming (Bonan 2008). On the other hand, forests provide an important climate forcing by attenuating the global warming by carbon sequestration and evapotranspiration (Bonan 2008). Furthermore, high vegetation is also the main source of reactive carbon in the atmosphere in form of Volatile Organic Compounds (VOCs), responsible for shaping the global tropospheric chemistry, altering the global carbon cycle, and regional photochemical oxidant formation (Fehsenfeld et al. 1992). Therefore, improving the knowledge on tall canopy-atmosphere interaction can contribute in better understanding of the climate system.

Although our knowledge on the interaction between the high vegetation and the atmosphere is improving, there is still a room for advancements. For example, large-scale atmospheric models are often employed to extend our understanding on canopy-atmosphere interaction and to investigate the forest-climate feedbacks on different spatio-temporal scales (e.g. Bonan 2015). Within these models the surface fluxes of energy, momentum, atmospheric constituents are parameterized, and often there are some discrepancies in their interpretation and application or comparison with observations. This becomes even more problematic when direct flux observations are not available, due to highly expensive eddy covariance instrumentation. As a result, the surface fluxes are usually inferred from mean profiles of the corresponding quantities based on the wellestablished flux-gradient relationships (or flux-profile relationships) (e.g. Kaimal and Finnigan 1994). Consequently, it is necessary to examine the representativeness and accuracy of surface fluxes calculated from mean profiles using these expressions in a parameterized form. One of our aims in this thesis is to improve the understanding of the high vegetation-atmosphere exchange processes, their quantification, and test methods for their parameterization. The next section elaborates how tall canopies affect the atmospheric flow within and above canopies, and how high vegetation influences the canopy-atmosphere exchange processes. 


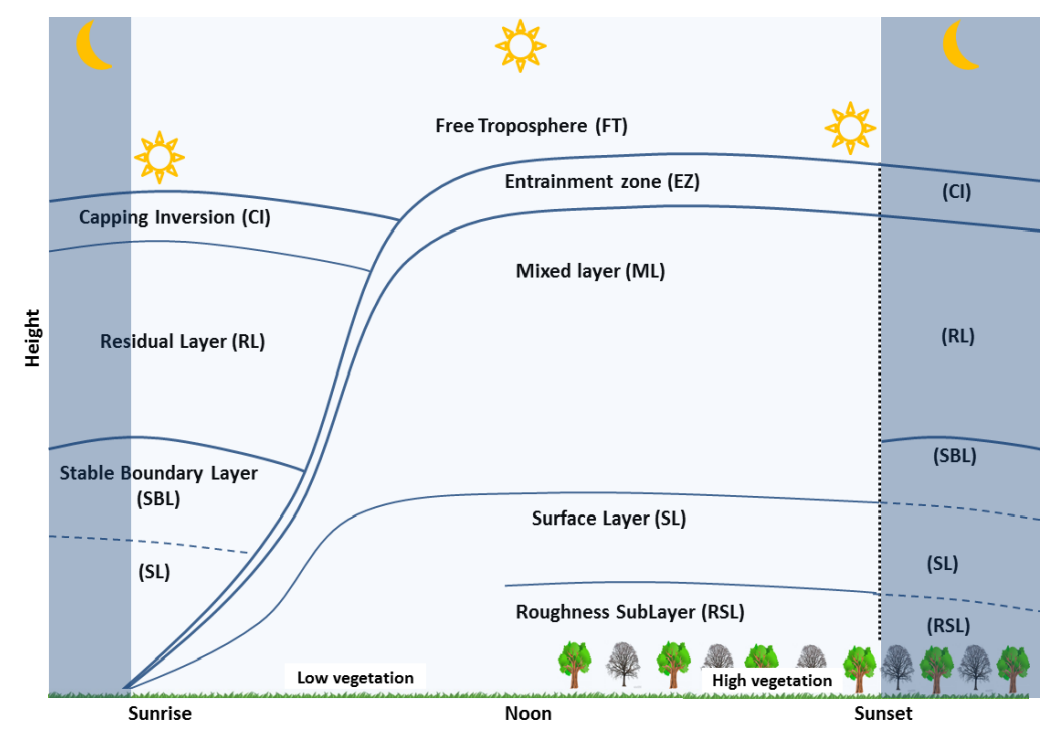

Figure 1.2: Diurnal structure of the planetary boundary layer over surface covered with low and high vegetation. This thesis mainly focusses on studying the high vegetation-convective boundarylayer interaction (from sunrise to sunset).

\subsection{The roughness sublayer within the land-PBL system}

Depending on the diurnal condition, the PBL is characterized by distinct regimes with different mixing (turbulent) properties. Relevant for our study, under unstable conditions, the strong exchange of heat and moisture between the surface and the atmosphere, driven by the buoyant thermal plumes as a results of solar heating on the earth's surface, leads to convective (thermal) turbulence. The PBL under these conditions is called the convective boundary layer (CBL) (Stull 1988).

The CBL is characterized by a mixed layer (Fig. 1.2) above the surface, in which the thermodynamic boundary-layer state variables (Lilly 1968; Tennekes and Lumley 1972) and the atmospheric reactants (Ouwersloot et al. 2012) are well mixed. Above the CBL there is the residual layer during the morning transition and the free troposphere (FT), a stably stratified layer (Fig. 1.2). Through the interface between the well-mixed layer and the free troposphere, the entrainment zone (EZ), both layers still exchange energy, momentum and atmospheric constituents (illustrated by the horizontal dashed red line in Fig. 1.3a). At the other relevant interface, and in direct contact to the surface, the well-mixed atmospheric quantities adjust to the surface conditions in the lower atmosphere. This layer is characterized by a relatively constant turbulent flux profile (e.g. Businger et al. 1971) in height and is called the atmospheric surface layer (ASL) 
(Fig. 1.2). Normally, the atmospheric SL extends to around 5-10\% of the CBL depth (e.g. Stull 1988).

In the ASL, the turbulent structure is determined by three quantities: height, friction velocity and buoyancy flux (primarily the sensible heat flux). Based on the surface similarity theory (Monin-Obukhov similrarity theory (MOST), Monin and Obukhov 1954; Foken 2006), from these three quantities one dimensionless stability parameter can be derived:

$$
\zeta=\frac{z}{L}
$$

where $z$ is the height above the surface and $L$ is the Obukhov length (see Chapter 2), a scale of the surface layer that indicates at which height the effect of buoyancy is bigger than to that of shear (Zilitinkevich 1970). The atmospheric quantities related to the surface turbulence, and made dimensionless with appropriate turbulent scales, are a function of only this atmospheric stability parameter. Similarity theory states that the stability effects become negligible when the buoyancy flux approaches zero $(L \rightarrow \pm \infty)$, but also when approaching the surface $(z \rightarrow 0)$.

Relevant to this study is that surface fluxes are related to the mean profiles of the corresponding quantities with 'universal' functions that depend only on the dimensionless atmospheric stability parameter. In differential form this reads:

$$
\frac{k z}{\varphi_{*}} \frac{d \varphi(z)}{d z}=\phi_{\varphi}(\zeta),
$$

where $\varphi$ represents the mean variable of interest (e.g. velocity, temperature, humidity, chemical reactants), $\varphi_{*}$ is a characteristic scale of a variable defined as the vertical turbulent flux divided by the friction velocity $u_{*}$ (with a minus sign), $\kappa$ is the von-Kármán-constant ( $\kappa=0.41$, based on empirical measurements) (Högström 1988); $\phi$ are 'universal' functions in the surface layer, which are dependent only on the atmospheric stability $\zeta$ (Högström 1988).

An example for a profile of the wind speed in the surface layer over relatively tall and dense canopy is presented in Fig. 1.3b with the dashed black line. This profile follows a semilogarithmic law and depends mainly on the height and the atmospheric stability. It is important to note here that the MOST is applied by assuming the surface as a geometrically rough wall, for which the surface is displaced at certain height in the canopy (relative to the ground surface) at which all momentum is expected to be absorbed by the roughness elements. Consequently, the mean wind speed at that (and below) displacement height is expected to be zero (Fig. 1.3b). The presence of high vegetation however influences the atmospheric flow at the lower part of the ASL near the surface and therefore modifies the turbulent structure of the flow. This layer is named the roughness sublayer (RSL) (Fig. 1.2). The flux-profile relationships that are normally assumed to be valid in the surface layer are modified due to the altered turbulence structure (e.g., Thom et al. 1975; Chen and Schwerdtfeger 1989). 


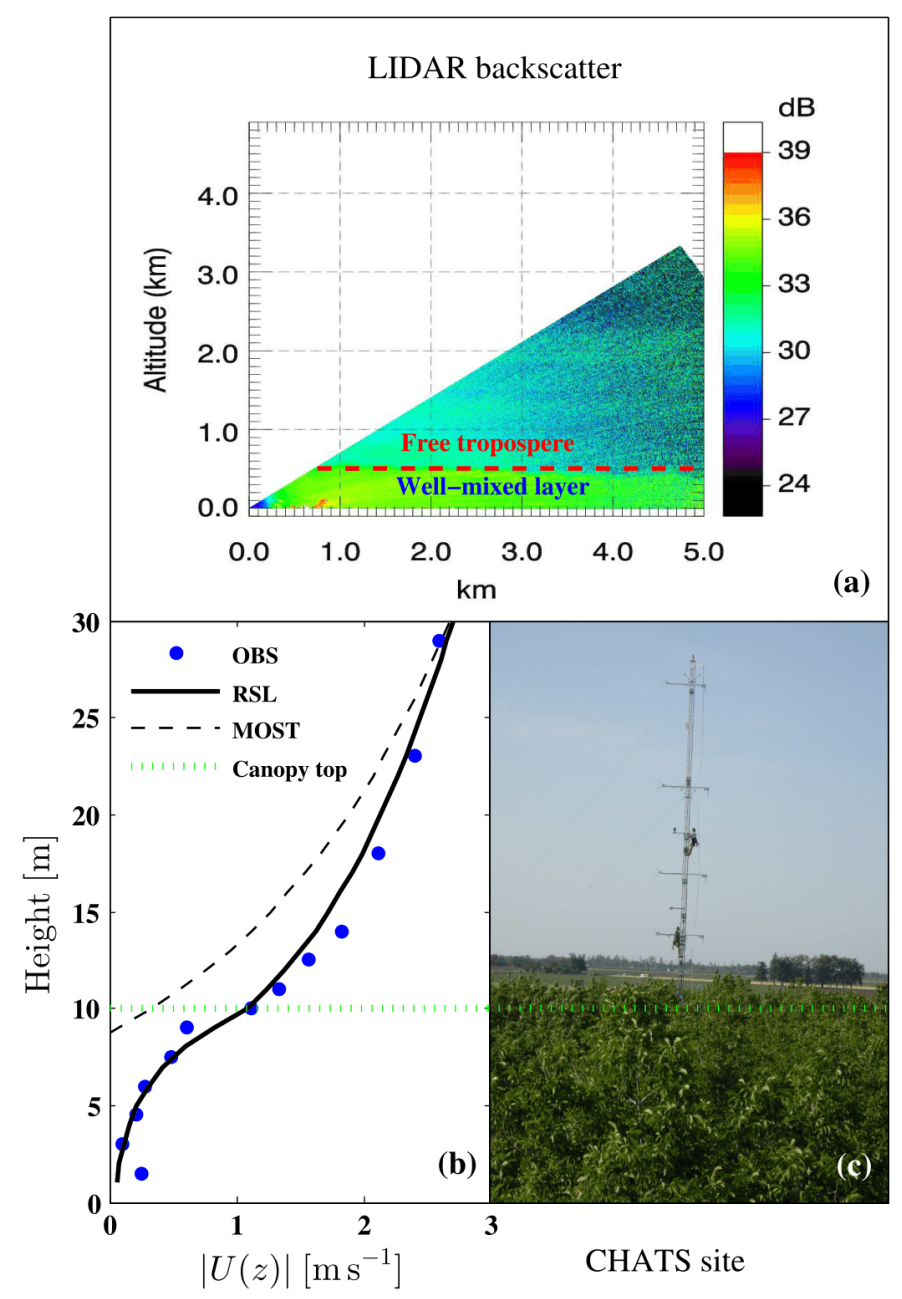

Figure 1.3: An overview of some of the methods and the concepts used in this thesis combining observational evidence and modelling; (a) data from the LIDAR backscatter signal operating at Chanopy Horizontal Array Turbulence Study (CHATS) site on the 27th of May 2007 at 12:00 LT (19:00 UTC); (b) profiles of observed and modelled wind velocity at CHATS for the same period and time; the modelled profiles are based on standard similarity theory (Monin and Obukhov 1954) (dashed line) and its modification by Harman and Finnigan (2007); (c) the CHATS canopy (site) and the $30 \mathrm{~m}$ tall vertical measurement tower. 
Based on observational evidence, the height of the RSL is usually 2-3 canopy heights (e.g., Thom et al. 1975; Raupach 1979; Garratt 1980; Högström 1988; Högström et al. 1989; Cellier and Brunet 1992; Högström 1996; Simpson et al. 1998; Mölder et al. 1999; De Ridder 2010; Shapkalijevski et al. 2016a).

To explain the altered mixing in and above the canopy, Raupach et al. (1996) postulated that the canopy's vertically distributed momentum sink induces an inflection point in the vertical profile of mean velocity at the canopy's vicinity. This is supported and corroborated by a number of observed profiles of the mean wind velocity within and above the canopy (see for example the blue circles in Fig. 1.3b). This differs from the prototypical surface layer wind profile in which the velocity typically varies logarithmically (under neutral conditions) with height. Thus, the flux-profile relationship in Eq. (1.2) requires a modification.

Finnigan et al. (2009) confirmed and extended the hypothesis developed by Raupach et al. (1996) and showed that the inflection-point instability generates turbulent organized structures, resulting in sweeps and ejection motions in and above the canopy, which are the drivers of the mixing near the canopy top.

Both Raupach et al. (1996) and Finnigan et al. (2009) suggested that the extent of the vertical velocity shear at the canopy top, expressed as the vorticity thickness $\left(\delta_{\omega}=u /[d u / d z]\right)$, controls the depth over which the organized motions impact the flow. This vorticity thickness defines a proper scale for the depth of the RSL height. Harman and Finnigan (2007) developed parameterizations for the exchange processes within the roughness sublayer taking into account the effects of the tall canopy in the MOST flux-profile relationships via the roughness sublayer function $\hat{\phi}\left(\zeta, \delta_{\omega}\right)$ :

$$
\frac{k z}{\varphi_{*}} \frac{d \varphi(z)}{d z}=\phi_{\varphi}(\zeta) \hat{\phi}\left(\zeta, \delta_{\omega}\right) .
$$

This resulted in improved representations of the profiles of the wind velocity within and above the tall canopy (Harman and Finnigan 2007) (also shown by the solid line in Fig. 3b). Later this theory has been extended to scalar transport within and above tall canopies (Harman and Finnigan 2008). Omitting these roughness sublayer effects on the flux, parameterization from observed mean profiles just above the canopy results in significant underestimation of the inferred vertical turbulent fluxes (e.g. Mölder et al. 1999; De Ridder 2010). Moreover, in order to study the high vegetation-atmosphere exchange processes on larger spatio-temporal scales, seminal studies have introduced the roughness sublayer effects in flux-gradient relationships of the thermodynamic variables in the surface schemes of numerical atmospheric models (Physick and Garratt 1995; Harman 2012).

This thesis extends the knowledge obtained in the previously mentioned studies. We therefore contribute to improve our understanding and representation of the roughness sublayer-planetary boundary-layer system. Our focus will not be on thermodynamic properties only, but we will also study exchange of chemical species. The next section gives a general overview on the main 
methods and concepts used in this thesis.

\subsection{Observed and modelled turbulent fluxes in and above canopies}

\subsubsection{Measuring turbulent fluxes: CHATS a comprehensive experiment}

In this thesis, we have extensively used the comprehensive dataset from the Canopy Horizontal Array Turbulence Study (CHATS) campaign (Patton et al. 2011). CHATS is an unique experiment aiming to contribute in the understanding of the high vegetation (orchard)-atmosphere exchange processes by connecting high quality multi-level horizontal and vertical observations with model development and testing (Patton et al. 2011).

There are several reasons why we chose the CHATS dataset as one of the main methods within this study. Combined high-quality measurements of the thermo(dynamics) and chemistry is the first reason. This gives us opportunity to study the thermo(dynamics) and the chemistry of the CBL in an integrated manner (e.g. Ouwersloot et al. 2012; van Stratum et al. 2012). Another reason to use the dataset from this experiment is related to the canopy homogeneity in combination with the observed, relatively constant- wind direction, which allows a well-developed roughness sublayer above the canopy. This is convenient for studying canopy-atmosphere interaction in an 'idealized' way, since an irregular shape and distribution of the canopy would bring additional uncertainty in the turbulence structure within and above the canopy (Raupach et al. 1996; Finnigan et al. 2009). In addition, the availability of observations collected on seasonal bases (before and after leafout) is a motivation to investigate the role of canopy phenology on turbulence parameterization within the roughness sublayer (Dupont and Patton 2012a; 2012b).

The CHATS experiment took place in walnut orchard fields in the Sacramento valley near Dixon, California (The USA) from 15 March until 12 June 2007. The orchards location was chosen to meet the previously set criteria of a homogeneously-distributed and relatively tall canopy, as well as consistent wind direction and velocity (Patton et al. 2011). The matured orchards trees ( 25 years old) were regularly distributed with similar distance between them of around $7 \mathrm{~m}$ (measured as distance between stems) and average height of $10 \mathrm{~m}$ (Fig. 1.2c), which makes this canopy relatively sparse.

During the CHATS campaign, observations were collected from several instrumentation arrangements. A single 30th-meters tall tower (see Fig. 1.2c) was used to measure mechanical and thermodynamic turbulent fluxes within and above canopy using the EC technique. Furthermore, the setup provided profiles of mean wind speed, temperature, water vapour, $\mathrm{CO}_{2}$ concentrations, and concentrations of reactants such as: ozone, $\mathrm{NO}, \mathrm{NO}_{x}$, and several VOCs from a biological origin (Acetone, Benzene, Toluene, Monoterpenes and Methyl Salicylate (MeSA)) (Karl et al. 2008) were also measured at the several heights below and above the canopy top (Patton et al. 2011). To complete these measurements, the remaining components of the radiation balance, incoming and outgoing shortwave and longwave radiation (see Chapter 3 for more details), were 
also measured at the tower.

Measurements of the soil properties (e.g. moisture, heat flux, diffusivity, conductivity and heat capacity) were collected at $0.05 \mathrm{~m}$ below the ground. Relevant for our study, and used to connect surface-canopy exchange with the development of the boundary layer, were the observations from the high-resolution aerosol backscatter lidar remote sensor (Raman-shifted Eye-safe Aerosol Lidar, REAL), since we are interested in integrating canopy scale with boundary-layer scale turbulence parameterization. The REAL was measuring the aerosol content (dispersion) on canopy scale, orchard scale and larger scales (up to $5 \times 5 \times 5 \mathrm{~km} 3$, in vertical, longitudinal and latitudinal directions). Based on these observations, we were able to retrieve useful information on the dynamics of the atmospheric boundary-layer depth (see Appendix 3C). A full description of the CHATS experiment, its motivation, site, observations and instrumentation can be found in Patton et al. (2011). More detailed information about the CHATS data used in this thesis is presented in Chapters 2-4.

The main limitations in our study, connected with the use of this dataset, are not having information on the boundary-layer state at the entrainment zone and limited observations on the chemistry. The CHATS boundary-layer structure and dynamics are affected by the atmospheric flows driven by regional topography and/or the atmospheric fronts from the see area, which often enter the California Central valley (Bianco et al. 2011; Mayor 2011). A discussion about these complications and their effects on the results in this study is presented in Chapters 3, 4 and 5 . A discussion about the sparsity of the orchard canopy and its impact on the turbulent exchange processes within the RSL is presented in Chapter 2 and 5.

\subsubsection{Parameterizing turbulent fluxes}

A suite of numerical techniques exists to predict flow fields and vertical turbulent fluxes within and above forest canopies. One must balance the computational costs of the predicted solutions with its correctness in representing the turbulent processes under investigation.

If turbulent motions are not resolved, the turbulent fluxes in the governing equations of the state variables and atmospheric compounds within and above the canopy need to be parameterized using turbulence closure. Several mathematical techniques exist to parameterize these fluxes (Wilcox 1998; Wyngaard 2010). The turbulence parameterizations can be categorized in two main groups depending on the nature of the turbulence: non-diffusive (or non-local) and diffusive (or local) closure methods (Stull 1988).

When non-local closure is applied, the unknown fluxes of a certain quantity are parameterized by gradients and other known quantities at many points in space (and thus non-local), analogous to dispersion (Lagrangian dispersion theory) or advection (Transilient turbulence theory) processes (Stull 1988). According to these theories, the turbulent fluxes of scalars are calculated from their source and sink vertical distributions, as integrated over height. The source and sink vertical distributions are predicted from the observed concentration and turbulent fields, charac- 
terized by concentration profiles and vertical velocity variation that changes in time (for more details see Stull 1988). This type of turbulence closure parameterization is usually convenient when calculating fluxes within high vegetation, where often the turbulent transport is affected by non-diffusive (non-local) turbulence causing counter-gradient transport (Denmead and Bradley $1985 ;$ 1987). One of the most commonly used methods to parameterize fluxes within canopies, considering the non-local turbulence effects, was developed by Raupach (1989a, 1989b). By using the Lagrangian fluid mechanics theory, Raupach (1989a, 1989b) developed the so called Inverse Near-Field method to calculate the turbulent fluxes within a tall canopy from known velocity and concentration fields. This method is presented in more detail in Chapter 4.

When local-closure methods are applied, the unknown turbulent fluxes are parameterized by gradients and other known quantities at the same point (locally), analogous to molecular diffusion. Based on these methods, vertical turbulent fluxes of the atmospheric state variables and constituents within the RSL and ASL are related to the mean gradients of those quantities and a turbulent transfer coefficient that accounts for the intensity of mixing. More complex closure approaches (second- or higher-order closure) are computationally costly and therefore have relatively limited applicability (e.g. Wilson and Shaw 1977; Meyers and Paw 1986; Wilson 1988). The turbulence closures based on turbulent kinetic energy, its dissipation, and a turbulent length scale (one-and a half-order closure) have been shown to have satisfactorily accuracy and relatively large applicability (e.g. Sogachev et al. 2002; Katul et al. 2004; Sogachev et al. 2012). However, the most commonly used turbulence closures in the large-scale atmospheric models (e.g. Weather Research \& Forecasting model (WRF), Integrated Forecasting System (IFS)) are the turbulence closures of the first-order type. Based on first-order closure methods, the turbulent transfer coefficient is characterized by a turbulent (mixing) length scale, velocity scale (friction), and a functions accounting for the atmospheric diabatic stability (Monin and Obukhov 1954) and canopy effects (Sect. 1.2) (Physick and Garratt 1995; Harman and Finnigan 2007; 2008).

Although the effects we are interested in act on small scales and require turbulence-resolving models to be investigated, they can also affect the larger-scale state of the atmosphere and therefore need to be parameterized in larger-scale models. Therefore, this thesis focusses on studying and evaluating the parameterization of these small-scale turbulent processes as used within the large-scale models (up to one-and-a-half-order turbulence closure complexity). By integrating the knowledge from the previous studies on flux parameterization in the roughness sublayer (e.g. Harman and Finnigan 2007; 2008) and flux tower observations, we study in detail how the turbulence parameterization should be modified due to the presence of a tall canopy, and how this impacts the CBL dynamics and chemistry. Moreover, to account for the non-diffusive character of the turbulence and its effect of the vertical fluxes within the canopy, we use the non-local closure method based on the Lagrangian dispersion theory (Raupach 1989a; 1989b), which will be further outlined in Chapter 4. The next section (Sect. 1.4) gives an overview of the research questions of this thesis and the strategy how to answer these questions. 


\subsection{Research questions, strategy and outline}

Based on the above-elaborated motivation to study the tall canopy-CBL interaction, within this thesis we have formulated three general research questions. These questions are investigated in Chapter 2 to 4 adopting different suitable research strategies:

- Question 1: What are the effects of canopy phenology and atmospheric stability on turbulent exchange of energy and momentum within the roughness sublayer?

In Chapter 2, we investigate whether and how the canopy phenological changes and the atmospheric stratification affect the turbulence parameterization closures. We study representative turbulence formulations of the turbulent exchange coefficients, as implemented in a number of atmospheric models. We focus on the exchange of momentum, heat and moisture, as well as on turbulent kinetic energy within the roughness sublayer. By applying an additional function in the MOST formulations for the turbulent exchange coefficient to account for the canopy effects on the atmospheric flow within the RSL (Harman and Finnigan 2007; 2008), we valuate these new formulations under leaf and non-leaf conditions and different atmospheric stability classes. We validate all turbulence formulations under the analysis by using the observations from the CHATS experiment at three heights above the canopy.

- Question 2: What is the impact of the roughness sublayer in representing the dynamics of the convective boundary layer?

Based on the CHATS observations, we select representative days to study the impact of the roughness sublayer in modeling the dynamics of the convective boundary layer. To this purpose, we modify the surface scheme of an atmosphere mixed-layer model (van Heerwaarden et al. 2009) by integrating a roughness-sublayer representation following the recommendations by Harman (2012) to account for the tall-canopy effects in the parameterization formulations of the turbulent surface fluxes. We use the mixed-layer model because of its simplicity (Lilly 1968; Tennekes and Driedonks 1981) and optimal robustness (Vilà-Guerau de Arellano et al. 2015), which allows us to perform a large number of systematic case studies and thorough sensitivity analysis on the integrated roughness sublayer-CBL system with low computational costs. Moreover, the mixed-layer model has been shown to be a powerful tool when studying land-atmosphere (thermos-)dynamics interaction (e.g. van Heerwaarden et al. 2009), as well as effects between boundary-layer dynamics and chemistry (Vilà-Guerau de Arellano et al. 2009; Ouwersloot et al. 2012; van Stratum et al. 2012; Janssen et al. 2013). In view of the complexity of the flow, influenced not only by canopy effects but also by valley-mountain and see-breeze flows, we design a systematic approach to determine the different contributions of those effects to the budgets of potential temperature, specific humidity and wind speed. 
- Question 3: What is the contribution of physical and chemical processes to the ozone budget above the CHATS canopy, specifically focusing on ozone surface deposition?

The knowledge on the CBL dynamics over the CHATS site obtained in Chapter 3 is used in Chapter 4 to study the mixed-layer ozone budget for the CHATS site. The same case study and model set up is used to investigate and quantify the role of different contributors to the total budget of ozone. Special attention is placed on the role of the ozone deposition onto the canopy and its effect on the ozone budget. Since no direct fluxes of ozone were observed during CHATS, and these are needed as lower boundary conditions in the model, it is necessary to calculate the deposition velocity of ozone to parameterize its fluxes. Therefore, crucial attention is given to the calculation of the ozone fluxes and deposition velocities from the available observed ozone concentration profiles during CHATS.

A general discussion about the assumptions, limitations and the results presented in this research is given in Chapter 5, accompanied with perspective and recommendations. Finally, in Chapter 6 the main results of this thesis are summarized. 


\section{2}

\section{Influence of canopy seasonal changes on turbulence parameterization within the roughness sublayer over an orchard canopy}

In this observational study, the role of tree phenology on the atmospheric turbulence paramet-
erization over $10 \mathrm{~m}$ tall and relatively sparse deciduous vegetation is quantified. Observations
from the Canopy Horizontal Array Turbulence Study (CHATS) field experiment are analysed to
establish the dependence of the turbulent exchange of momentum, heat and moisture, as well as
kinetic energy on canopy phenological evolution through widely used parameterization models
based on: a) dimensionless gradients, or b) turbulent kinetic energy (TKE) in the roughness
sublayer. Observed vertical turbulent fluxes and gradients of mean wind, temperature and
humidity, as well as velocity variances, are used in combination with empirical dimensionless
functions to calculate the turbulent exchange coefficient. The analysis shows that changes in
canopy phenology influence the turbulent exchange of all quantities analysed in this study.
The turbulent exchange coefficients of those quantities are twice as large near the canopy top
for leafless canopy then for full-leaf canopy under unstable and near-neutral conditions. This
turbulent exchange coefficient difference is related to different penetration depth of the turbu-
lent eddies organized at the canopy top, which increases for a canopy without leaves. The

This chapter is published as Shapkalijevski et al. (2016) 
TKE and dissipation analysis under near-neutral atmospheric conditions additionally shows that TKE exchange increases for a leafless canopy due to reduced TKE dissipation efficiency compared to that when the canopy is in full-leaf. The study closes with discussion surrounding the implications of these findings for parameterizations used in large-scale models.

\subsection{Introduction}

Turbulence above tall vegetation depends strongly on tree phenology, and more specifically on the presence of leaves and branches that influence the atmospheric flow in and above the canopy (Dupont and Patton 2012a; 2012b). Since turbulence performs the majority of the vertical exchange of momentum, mass, energy, and trace gases (Gao et al. 1989), the dependence of turbulence parameterization on canopy seasonal changes should be included in weather and climate models (Fitzjarrald et al. 2001). To capture the vertical exchange of momentum, energy and trace gases, it is crucial to determine how the flux-gradient relationships and turbulent kinetic energy are influenced by leaf state and atmospheric stability (Thom et al. 1975; Raupach 1979; Denmead and Bradley 1985; Högström et al. 1989).

Regarding the flux-gradient relationships, it has been recognized that the standard MoninObukhov Similarity Theory formulations (MOST, Monin and Obukhov 1954) are only valid at heights well above a rough vegetated surface (Högström 1996). The atmospheric layer in which the flux-gradient relationships are directly impacted by the canopy is called the roughness sublayer (RSL). It is in this layer that numerous studies over evergreen forests confirm that the heat exchange is more efficient than in the inertial surface layer above (Thom et al. 1975; Raupach 1979; Denmead and Bradley 1985; Högström et al. 1989). However, those studies failed to converge on a single relationship to describe momentum exchange above tall canopies, suggesting that additional physical processes must affect the exchange. The pioneering study by Thom et al. (1975) and later investigations by Raupach (1979) and Denmead and Bradley (1985) indicated that dimensionless gradients for momentum over tall canopies are nearly identical to those over low vegetation. Contrary to this, independent studies over a savannah (Garratt 1980), in a wind-tunnel (Raupach et al. 1986), and over a pine forest (Högström et al. 1989), indicate that the turbulent momentum exchange is more efficient over rough surface, implying a reduction in momentum dimensionless gradients close to the canopy top (Högström et al. 1989).

To explain the increased mixing efficiency in the canopy's vicinity, Raupach et al. (1996) put forward what has become known as the mixing-layer analogy, where they postulated that the canopy's vertically distributed momentum sink induces an inflection point in the vertical profile of mean streamwise velocity $(\bar{u})$ - a feature that is unique compared to traditional surface layer flow in which the streamwise velocity typically varies logarithmically with height. Finnigan et al. (2009) used large-eddy simulation (LES) to extend Raupach et al. (1996) mixing-layer analogy by showing that the inflection-point instability generates turbulent organized structures, combination 


\subsection{INTRODUCTION}

of head-up and head-down hairpin shaped rolls, that are responsible for the enhanced mixing near canopy top. In combination, Raupach et al. (1996) and Finnigan et al. (2009) theory suggests that the extent of the vertical streamwise-velocity shear at canopy top, expressed as the vorticity thickness, $\delta_{\omega}=\bar{u} /[d \bar{u} / d z]$, controls the depth over which the organized motions impact the flow and, subsequently, the depth of the RSL. In developing their RSL parameterization, Harman and Finnigan (2007) were able to improve predicted flux-gradient relationships over a range of canopy types compared to traditional MOST.

Over the years, relatively few studies have focused on the modification of turbulence resulting from the seasonal variation of deciduous forests. Bohrer et al. (2009) and Maurer et al. (2013; 2015), using observations and large eddy simulations, showed that accounting for the seasonal and interannual variation of roughness length and displacement height, both functions of the canopy structure, yield more precise and less biased estimates for friction velocity than models with temporally invariable parameters. Both, Bohrer et al. (2009) and Maurer et al. (2013; 2015) noted the need for further investigation of 'canopy structure-roughness dependence' and its effect on the flux-gradient relationships. The Dupont and Patton (2012a; 2012b) study used the Canopy Horizontal Array Turbulence Study (CHATS) dataset to stress the importance of canopy leaf state on the transport of momentum, heat and moisture within and above a deciduous walnut orchard. Concentrating on turbulent coherent structures at the canopy-atmosphere interface, Dupont and Patton (2012a; 2012b) analysis suggested that "the canopy's seasonal state plays a vital role in determining the turbulent transport processes coupling the canopy layers with the overlying atmosphere" (Dupont and Patton 2012a). Here we extend on their analysis by investigating the impact of seasonal variation of a deciduous canopy on turbulence quantified through the flux-gradient relationships and the TKE. More specifically, we systematically investigate phenology's effect on the turbulent exchange coefficient, $K$, within the RSL. Subsequently, we investigate whether commonly used $K$-parameterizations can mimic the observed behavior of $K$. $K$-parameterizations usually hinge on dimensionless gradients of momentum, heat and moisture (1st - order closure), or on TKE $(e)$, and its dissipation $(\varepsilon)$ (1.5-order closure). 1st - order closure models are commonly used in large-scale atmospheric models (e.g. WRF, ECMWF-IFS), while the 1.5-order closure models have become useful tool to model turbulent flow over tall canopies for a variety of special and temporal scales (e.g. Sogachev et al. 2002; Katul et al. 2004; Sogachev et al. 2012).

Our investigation aims to answer three research questions:

1. What is the effect of canopy phenology on the exchange of momentum, heat and moisture within the RSL over deciduous vegetation?

2. How well do $K$ - parameterizations based on different physically-based turbulence closure assumptions represent the observed behaviour of $K$ and its dependence (if any) on the

Note that WRF also uses one-and-a-half closure formulation in addition to 1st - order closure (Skamarock et al. 2005.) 
evolution of canopy phenology and atmospheric stability?

3. How sensitive are the dimensionless gradients and $K$-values to displacement height variations resulting from canopy phenology and atmospheric stability?

Our methodology combines a comprehensive observational data set and conceptual modelling. We analyze the observations obtained on a $30 \mathrm{~m}$ tower during the CHATS experiment (Patton et al. 2011), which took place in Spring 2007 in and above a $10 \mathrm{~m}$ tall deciduous walnut orchard in Dixon, California, USA, before and after leaf-out. Analyzing the dependence of the turbulence parameterization on canopy phenological changes above a canopy over a season is a key novelty of this study.

Interpretation and discussion of the CHATS observations is supported by using: (i) empirically MOST-based dimensionless flux-gradient functions from literature (Dyer 1974; Högström 1988) and (ii) RSL-adapted dimensionless gradients for momentum (Harman and Finnigan 2007; hereafter HF07) and scalars (Harman and Finnigan 2008; hereafter HF08).

\subsection{Theory}

Gradient transport theory ( $K$-theory) relates the vertical turbulent fluxes of vector and scalar quantities in the atmospheric surface layer to the corresponding mean gradients through the turbulent exchange coefficients $K$ (Stull 1988):

$$
\overline{w^{\prime} \psi^{\prime}}=-K_{\psi} \frac{\partial \bar{\psi}}{\partial z}
$$

where $\overline{w^{\prime} \psi^{\prime}}$ refers to momentum $\left(\overline{w^{\prime} u^{\prime}}\right)$, heat $\left(\overline{w^{\prime} \theta^{\prime}}\right)$ or moisture $\left(\overline{w^{\prime} q^{\prime}}\right)$ fluxes, the mean $\bar{\psi}$ represents the mean wind speed $(\bar{u})$, potential temperature $(\bar{\theta})$, or specific humidity $(\bar{u})$ profiles and quantities with a' represent deviations from that mean; $z$ is the height from the ground. The $K$ defined in Eq. 2.1 is usually parameterized using dimensionless gradients $(\Phi)$ or from $e$ and $\varepsilon$. Below we give a brief theoretical overview of both approaches.

\subsubsection{Parameterization of $K$ based on dimensionless gradients}

In a coordinate system with $z=0$ at the ground surface the turbulent exchange of momentum $(\psi=u)$, heat $(\psi=\theta)$ or moisture $(\psi=q)$ is parameterized as:

$$
K_{\psi}=\frac{\kappa\left(z-d_{\psi}\right) u_{*}}{\Phi_{\psi}},
$$

and

$$
\Phi_{\psi}=\frac{\kappa\left(z-d_{\psi}\right)}{\psi_{*}}\left(\frac{\partial \bar{\psi}}{\partial z}\right)
$$


where $u_{*}$ is the friction velocity, $d_{\psi}$ is the displacement height, which represents the mean source and sink height of $\psi$ ( is treated more fully in Sect. 2.1.2); and $\kappa$ is the von-Kármán-constant of 0.41 . Here $\psi_{*}$ represents the vector and scalar scales defined by $-\overline{w^{\prime} \psi^{\prime}} / u_{*}$. In MOST, the dimensionless gradients $\Phi_{\psi}$ (for momentum $\Phi_{m}$, heat $\Phi_{h}$ or moisture $\Phi_{q}$ ) are only a function of atmospheric stability, represented by the stability parameter $\zeta$, which is defined as:

$$
\zeta_{\psi}=\frac{\left(z-d_{\psi}\right)}{L}=\frac{g \kappa\left(z-d_{\psi}\right) \overline{w^{\prime} \theta_{v}^{\prime}}}{T_{v} u_{*}^{3}},
$$

where $T_{v}$ is a mean (reference) virtual temperature at the canopy top, $g$ acceleration due to gravity, $\overline{w^{\prime} \theta_{v}^{\prime}}$ represents the vertical buoyancy flux and $L$ is the Obukhov length. Note that for some parts of the analysis we define $\zeta_{c}$ at the canopy top $\left(\zeta_{c}=h_{c} / L\right)$, where $h_{c}$ is the average height of the trees observed in CHATS $\left(h_{c}=10 \mathrm{~m}\right)$ and $L$ is evaluated at canopy top.

We support our observational analysis on dimensionless gradients with empirically derived universal functions based on MOST (Dyer 1974; Högström 1988). The universal 'MOST' functions for momentum $\left(\Phi_{m}=\phi_{m_{M O S T}}\right)$ and heat and moisture $\left(\Phi_{h, q}=\phi_{h, q_{M O S T}}\right)$ are as follows:

$$
\begin{gathered}
\phi_{m_{M O S T}}= \begin{cases}(1-15.2 \zeta)^{-1 / 4}, & \zeta<0 \\
1+4.8 \zeta, & \zeta \geq 0\end{cases} \\
\phi_{h, q_{M O S T}}= \begin{cases}0.95(1-15.2 \zeta)^{-1 / 2}, & \zeta<0 \\
0.95+4.8 \zeta, & \zeta \geq 0 .\end{cases}
\end{gathered}
$$

\section{Modification within the RSL}

To account for the enhanced vertical mixing in the vicinity of a tall canopy HF07 and HF08 proposed a modification to standard MOST dimensionless gradients with the following multiplicative form:

$$
\Phi_{\psi}=\phi_{\psi_{M O S T}} \hat{\phi}_{\psi}
$$

In Eq. (2.7), $\hat{\phi}_{\psi}$ represents the effects of the canopy within the RSL. HF07 and HF08, derived expressions to quantify these effects, which read:

$$
\hat{\phi}_{\psi}=1-c_{1 \psi} \exp \left[-\frac{\beta c_{2 \psi}\left(z-d_{H F}\right)}{l}\right]
$$

where

$$
c_{1 \psi}=\left(1-\frac{\kappa S_{c}}{2 \beta}\right) \exp \left(\frac{c_{2 \psi}}{2}\right)
$$


In Eq. 2.9, $c_{1 \psi}$ represents a scaled amplitude of the RSL function, while $c_{2 \psi}$ is a scaled depth over which the RSL function operates. An elaborate expression for $c_{2 \psi}$ is described by Eq. (2.7) in Harman (2012), but for simplicity, we adopt HF07's 0.5 value. The displacement height in Eq. 2.8 is represented as follows:

$$
d_{H F}=h_{c}-d_{t},
$$

where $d_{t}=\beta^{2} L_{c}, L_{c}$ is canopy adjustment length scale (penetration depth), defined as:

$$
L_{c}=\left(c_{d} a\right)^{-1}
$$

where $c_{d}$ is a drag coefficient whose value is assumed to be 0.25 (HF07) and $a$ is the canopy's leaf area density which is assumed as constant with height ( $L A I / h_{c}$, HF07) to mimic application in a large-scale model. The Schmidt number, $S_{c}$, is stability dependent (Harman 2012): $S_{c}=$ $0.5-\tanh L_{c} / L$, while the mixing length, $l$, is parameterized as $l=2 \beta^{3} L_{c}$ (see HF07; Weligepolage et al. 2012). Finally, $\beta$ is a stability dependent variable that represents the ratio between the friction velocity and the mean wind speed at canopy top $\left(\beta=u_{*} / \bar{u}\right)$. Based on our analysis, we find that under weakly unstable, near-neutral and weakly stable atmospheric conditions $\beta$ has constant value of 0.3 , consistent with HF07. Under strong unstable conditions this variable increases up to 0.45 on average, while under strong stable conditions it decreases to nearly 0.2. Similar patterns of $\beta$ were observed in the data presented in the Harman's (2012) study. Since the majority of our data (around $80 \%$ ) belong to the stability classes where $\beta$ is independent of stability conditions, we adopt the value of $\beta=0.3$ in this study. Finally, the canopy adjustment length scale (Eq. 2.11) is defined as a measure of the distance over which a boundary layer with no prior knowledge of a tall canopy would need to equilibrate (adjust) to the presence of a canopy (Belcher et al. 2003; Harman and Finnigan 2007). $L_{c}$ (Eq. 2.11) is calculated from the CHATS dataset and compared to the parameterization $\left(L_{c}=4 h_{c} / L A I\right)$ of Harman and Finnigan (2007). The parameterization showed a satisfactory agreement with the observed $L_{c}$ under unstable and near neutral conditions. It is to be noted that the parameterization is questionable under strongly unstable and strongly stable conditions (Harman 2012), where the scatter in the observations is larger.

\section{Displacement height}

As mentioned, the displacement height (in Eqs. 2.2 - 2.3) can be interpreted as the mean source or sink height within the canopy. Based on the source distribution, $d_{\psi}$ can be defined through the following expression (Thom 1971; Jackson 1981):

$$
d_{\psi}=\frac{\int_{0}^{h_{c}} z S_{\psi} d z}{\int_{0}^{h_{c}} S_{\psi} d z},
$$


where the vertical source/sink distribution of the quantities within the canopy, $S_{\psi}$, is calculated as $S_{\psi}=\partial \overline{w^{\prime} \psi^{\prime}} / \partial z$. In our analysis we a priori adopt the assumption made in Raupach (1979) that the displacement height for heat and moisture equal the displacement height for momentum $\left(d_{m}=d_{h}=d_{q}\right)$. The reason for this assumption is further discussed in Sect. 2.5.2. Consequently, throughout this study we use the displacement height for momentum, $d_{m}$, as a unique variable, but we retain the dependence of $d_{m}$ on canopy phenology and atmospheric stability. The displacement height is used to calculate the turbulent length scale, $\kappa\left(z-d_{m}\right)$, and consequently the dimensionless gradients for momentum, heat and moisture (Eqs. 2.2 - 2.3). For completeness, we additionally compare predictions using a displacement height estimate that neglects any influence of canopy phenology or diabatic stability and is a specified fraction of canopy height (Thom 1971):

$$
d_{f i x}=0.76 h_{c}
$$

More information about the definition of the displacement height and its dependence on the canopy height, plant cover and wind velocity can be found in Foken (2008b).

\subsubsection{Parameterization of $K$ based on TKE and its dissipation}

Additionally, we analyze two commonly used turbulent closure formulations for $K$ (Sogachev et al. 2002; Katul et al. 2004; Sogachev et al. 2012) that are: (i) related to $e$ and the turbulent mixing length scale $\Lambda$ :

$$
K^{(i)}=c_{\mu} \Lambda e^{1 / 2}
$$

or (ii) to $e$ and $\varepsilon$ :

$$
K^{(i i)}=c_{\mu} \frac{e^{2}}{\varepsilon} .
$$

For the turbulent mixing length above the canopy we adopt the formulation used by Katul et al. (2004), which is similar to the one used in the dimensionless gradient analysis in this study in Sect. 2.2.1: $\Lambda=\kappa\left(z-d_{m}\right)$. We also adopt a value of 0.03 for the coefficient $c_{\mu}$ following Katul et al. (2004), since it has been tested for a variety of canopy types.

\subsection{Observations}

\subsubsection{CHATS experiment: Site and instrumentation}

The CHATS experiment took place in one of Cilker Orchards's walnut blocks in Dixon, California, USA. The site and instrumentation are described in detail in Patton et al. (2011) and Dupont and Patton (2012b) and summarized here. The observations were carried out in the spring of 2007. There were two intensive measurement periods defined by the canopy leaf state: one from 13 March to 15 April concentrating on the influence of the walnut trees before leaf-out (no leaf), 
and another from 13 May to 12 June focusing on the walnut trees after leaf-out (full leaf).

The measurements analysed in this study were obtained along a $30 \mathrm{~m}$ tower, which was located near the northern-most border of the section to ensure a fetch of about $1.5 \mathrm{~km}$ when focusing on southerly winds (see Fig. 1a and Fig. 3 in Dupont and Patton 2012b). Turbulent velocity components and air temperature fluctuations were measured at thirteen levels within and above the canopy using Campbell Scientific CSAT3 sonic anemometers sampling at $60 \mathrm{~Hz}$. The horizontal distribution of the vegetation was nearly homogeneous, where the average height of the trees $\left(h_{c}\right)$ was estimated to be $10 \mathrm{~m}$. Since our study focuses on turbulence above the canopy, we only analyse data from the seven above-canopy levels which were located at, $1.0 h_{c}, 1.1 h_{c}, 1.25 h_{c}$, $1.4 h_{c}, 1.8 h_{c}, 2.3 h_{c}$ and $2.9 h_{c}$ (see Fig. 7a in Dupont and Patton 2012b). Six NCAR-Vaisala hygrothermometers (TRH) measured profiles of temperature and relative humidity at $2 \mathrm{~Hz}$ at the same levels (with the exception of the level at $1.25 h_{c}$ ). Three Campbell Scientific KH20 krypton hygrometers sampled water vapour density fluctuations at $60 \mathrm{~Hz}$ at $1.0 h_{c}, 1.4 h_{c}$ and $2.3 h_{c}$. Finally, the vertical leaf area index (LAI) profile was measured using a Li-Cor LAI-2000. Varying with the phenology, the cumulative LAI increased from 0.7 (before leaf-out) to 2.5 (after leaf-out).

\subsubsection{Data processing}

The data from the sonic anemometers and hygrometers have been processed as follows. The sonic data, as available from the CHATS dataset, has been rotated using the planar fit method (Wilczak et al. 2001) based on 5-min average velocities. This rotation was applied for each period in which the mounting of the instrument under consideration remained unchanged. The rotation angles were based on nighttime data only, because these contain less noise than daytime data. Since wind at the sonic anemometer can be distorted by the nearby observational tower, data was excluded if wind originated from within 45 degrees of the tower. Before processing the raw turbulence data, short gaps (i.e. less than 4 seconds) in the raw data of the sonic anemometers and hygrometers were filled by linear interpolation. Next, the data from the slow TRH sensors was used to correct the mean level of the sonic temperature and the water vapour density obtained from the Krypton hygrometers. The actual temperature and specific humidity were derived from the sonic temperature and water vapour density at levels where both a sonic and a Krypton hygrometer were available. Since the specific humidity is used in our analysis (rather than water vapour pressure), a density correction (WPL, after the authors Webb, Pearman and Leuning (see Webb et al. 1980)) correction is not applied since its effect would be negligible (Lee and Massman 2011). Finally, incomplete vertical profiles of means, variances and covariances were filled by vertical interpolation, where the maximum size of a vertical gap was one instrument for a Krypton and two instruments for a sonic or a TRH sensor.

We analyze the behavior of the dimensionless gradients and TKE under a range of atmospheric stabilities. Following Dupont and Patton (2012b), we define the atmospheric stability classes based upon stability parameter, $\zeta_{m}$ (Table 2.1 ). We use an averaging time of $30 \mathrm{~min}$. for all 


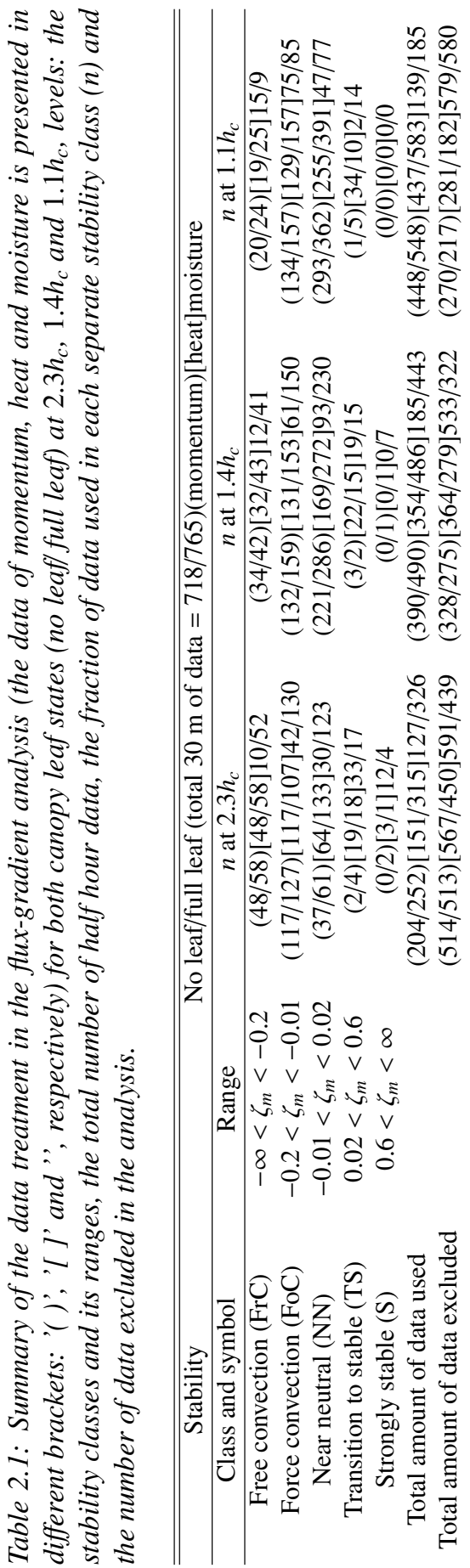


statistics and for both unstable and stable conditions. Dupont and Patton (2012a; 2012b) point out that for stable conditions it might be better to use shorter averaging times to reduce effects of non-stationarity. Since our analysis primarily focuses on unstable and near-neutral conditions, we consider $30 \mathrm{~min}$. satisfactory time average for the statistics. The recorded velocity components were rotated horizontally, such that $u$ represents the horizontal component along the mean wind $x$ direction, $v$ is the component along the $y$ direction, and $w$ represents the vertical component along the $z$ direction. To reduce the effect of statistical errors in derived quantities, the data were excluded when $u_{*}=0.2 \mathrm{~m} \mathrm{~s}^{-1}$ and $\left|\theta_{*}\right|<0.05 \mathrm{~K}$ (Garratt 1980). In addition, to provide a more precise calculation of the vertical derivatives, the vertical profiles of mean quantities $(u$, $\theta, q$ ) were smoothed using a three-point stencil in which the weight of the central point was twice the weight of the point below and above. The vertical gradients of wind, temperature and specific humidity were calculated at 3 levels above the canopy $\left(1.1 h_{c}, 1.4 h_{c}\right.$ and $\left.2.3 h_{c}\right)$, using finite differences based on the adjacent measurement levels. The canopy influence is anticipated to be largest at the level closest to canopy top, i.e. $1.1 h_{c}$, and the canopy influence is expected to have diminished significantly by $2.3 h_{c}$ level. The middle level $\left(1.4 h_{c}\right)$ is included to assess whether trends are monotonic with height. A summary about the information for the stability classes, the total number of half hour data, the fraction of data used in the analysis and the excluded data is presented in Table 2.1.

\subsection{Results and discussion}

\subsubsection{Phenology effects on the observed turbulent exchange coefficients}

Using CHATS observations, we are able to quantify the role of the phenology on the turbulent exchange coefficients above the deciduous canopy. Figure 1 shows the turbulent exchange coefficients for momentum $\left(K_{m}^{o b s}\right)$, heat $\left(K_{h}^{o b s}\right)$ and moisture $\left(K_{q}^{o b s}\right)$ calculated directly from the CHATS observations (Eq. 2.1) at three different heights above the canopy $\left(2.3 h_{c}, 1.4 h_{c}, 1.1 h_{c}\right)$ and as a function of atmospheric stability. Mean, and values have been grouped by stability classes (Table 2.1).

The uncertainties of $K_{m}^{o b s}, K_{h}^{o b s}$ and $K_{q}^{o b s}$, denoted by error bars, are calculated as two times the standard deviation within a stability class divided by the square root of the number of data samples. We find that $K_{m}^{o b s}, K_{h}^{o b s}$ and $K_{q}^{o b s}$ are strongly dependent on the canopy phenology under unstable (FrC and FoC) and near-neutral (NN) conditions and less dependent under stable conditions. The reason for the insignificant phenology influence on exchange coefficients under stable stratification could be that under stable conditions the decoupling between the canopy and the atmosphere is independent from canopy density/sparsity (Thomas and Foken 2007). Therefore, we focus our analysis on the stability range from $\operatorname{FrC}$ to NN. Figure 1 also shows that $K_{m}^{o b s}, K_{h}^{o b s}$ and $K_{q}^{o b s}$ for the leafless canopy are significantly larger (by a factor of two) than $K_{m}^{o b s}, K_{h}^{o b s}$ and $K_{q}^{o b s}$ for fully vegetated canopy, implying that vertical mixing is more efficient before leaf-out 


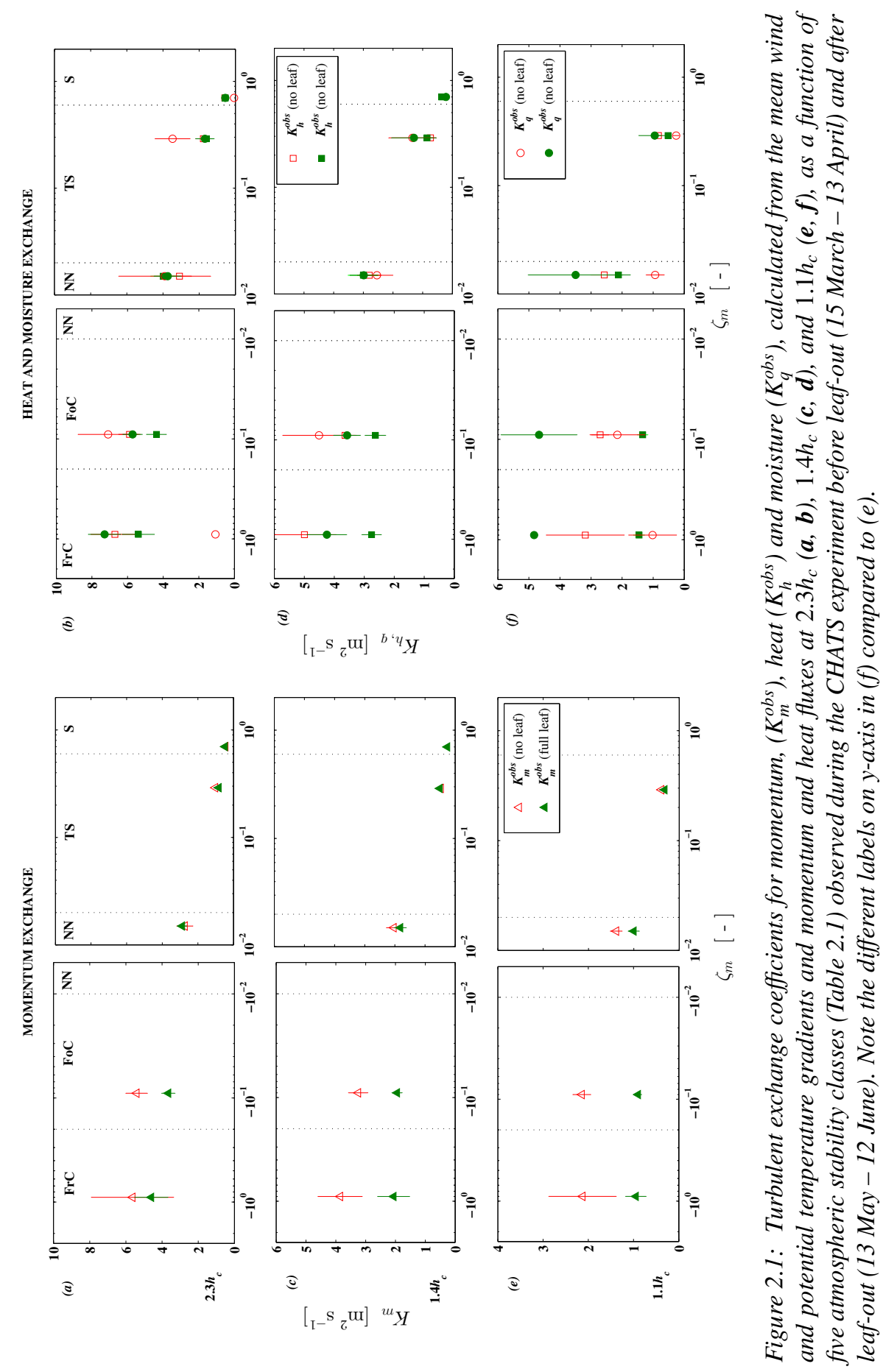


than after leaf-out above this deciduous canopy. These seasonal variations likely result from a number of reasons, such as: 1) deeper penetration of any turbulent coherent structures generated by the canopy-top shear instability process (Finnigan et al. 2009) when the canopy's more open, and 2) evolution of the vertical distribution of the available energy and its partitioning with the onset of leaf transpiration (Dupont and Patton 2012a; 2012b). As a consequence, the effect of the more open canopy on $K_{m}^{o b s}$ suggests that the scale of turbulence is larger than under conditions of full leaf. The heat and moisture transport both benefits from the larger scales in turbulence and from the fact that those larger scales are able to pick up heat and moisture that is further away (down) from the level of the gradient. As a result, we see that the enhancement of $K^{o b s}$ in the RSL is larger for heat and moisture than for momentum. In the following sections we systematically investigate how well different $K$-parameterizations (Eq. 2.2 and Eqs. 2.14 - 2.15) can capture observed $K_{m}^{o b s}, K_{h}^{o b s}$ and $K_{q}^{o b s}$ variations resulting from the evolution of canopy phenology and atmospheric stability.

\subsubsection{Phenology effects on $K$-parameterization}

\section{The role of the displacement height}

When calculating $K_{m}$ and $K_{h}$ based on the dimensionless gradients $\Phi_{m}$ and $\Phi_{h}$, the displacement height is an important length scale. To determine the sensitivity of $\Phi_{m}$ to the displacement height, we calculate $\Phi_{m}$ using the different displacement height calculations described in section 2.2.1 (see Table 2.2). We begin our analysis by showing under unstable and near-neutral conditions for both canopy leaf states (Fig. 2.2), where $\Phi_{m}$ is calculated using Eq. (2.3) at specific heights above the canopy $\left(2.3 h_{c}, 2.3 h_{c}, 2.3 h_{c}\right)$.

Remarkably, for each stability class the $\Phi_{m}$ calculated using the observed $d_{m}$ (Fig. 2.2a,c,e) is similar for both canopy configurations. Similar to this finding, Maurer et al. (2015) found insignificant sensitivity of the friction velocity to phenological changes when phenology dependent displacement height and roughness length were applied. However, using $d_{f i x}$, which does not account for the phenology changes, results in a smaller $\Phi_{m}$ for leafless canopy compared to $\Phi_{m}$ for the canopy with leaves (Fig. 2.2d,f). At heights closer to canopy top, $\Phi_{m}$ is very sensitive to the displacement height (Fig. 2.2e,f), while only small $\Phi_{m}$ differences are found at the height where the RSL blends with the ASL (Fig. 2.2a,b).

This analysis indicates that efforts utilizing dimensionless gradients to predict flux-gradient relationships above deciduous canopies should account for the seasonal evolution of the displacement height. As shown in Table 2.2 and Fig. 2.2, $d_{m}$ is up to $25 \%$ larger for a canopy in full-leaf conditions, compared to that without leaves (Table 2.2). Such variations have a large impact on the calculated dimensionless gradients at heights closer to canopy-top. For this reason, we recommend a displacement height formulation based on both $h_{c}$ and LAI (e.g., Raupach 1994; HF07), rather than a formulation that uses a constant fraction of $h_{c}$ (e.g. Simpson et al. 1998). Further- 


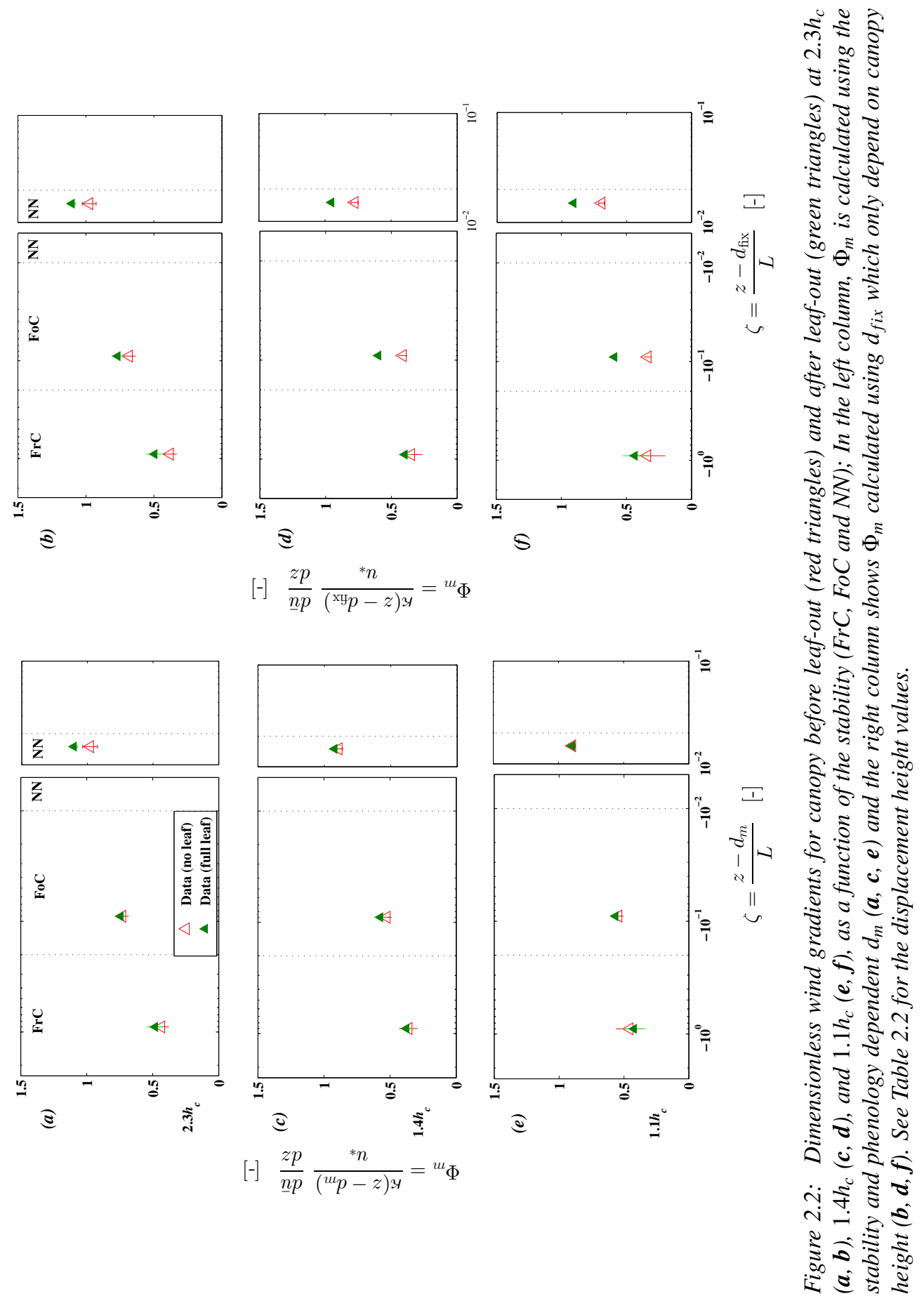


more, the weak dependence of the displacement height on stability (Table 2.2) does not result in significant changes in the dimensionless gradients at levels well above the canopy $\left(1.4 h_{c}\right.$ and $2.3 h_{c}$ ). However, the $0.04 \mathrm{~m}$ difference in $d_{m}$ between FoC and NN for full-leaf canopy (Table 2.2) produces approximately a $13 \%$ difference in $\Phi_{m}$ at $1.1 h_{c}$ : a variation substantial enough to corroborate HF07 and HF08's suggestion that the displacement height should vary with diabatic stability, which is introduced through $\beta$ in $d_{t}$ (see HF07 and HF08).

Table 2.2: Observed displacement height for momentum, $d_{m}$, and its standard deviation, std $\left(d_{m}\right)$, normalized by $h_{c}$ (Eq. 2.12); $d_{\text {fix }}$ only as a constant fraction of the canopy height (Eq. 2.13).

\begin{tabular}{l|c|c|c|c}
\hline \hline Leaf state & $\frac{d_{m}}{h_{c}} \pm \frac{\operatorname{std}\left(d_{m}\right)}{h_{c}}(\mathrm{FrC})$ & $\frac{d_{m}}{h_{c}} \pm \frac{\operatorname{std}\left(d_{m}\right)}{h_{c}}(\mathrm{FoC})$ & $\frac{d_{m}}{h_{c}} \pm \frac{s t d\left(d_{m}\right)}{h_{c}}(\mathrm{NN})$ & $d_{m} / h_{c}$ \\
\hline No leaf & $0.57 \pm 0.02$ & $0.58 \pm 0.01$ & $0.6 \pm 0.02$ & 0.76 \\
Full leaf & $0.76 \pm 0.03$ & $0.79 \pm 0.02$ & $0.75 \pm 0.02$ & 0.76 \\
\hline
\end{tabular}

\section{The role of foliage on $K$-parameterization using dimensionless gradients}

Traditional MOST To study the dimensionless gradients' departures from their MOST values, first we analyze the validity of the constant-flux hypothesis. For that reason, we analyze the divergence of the vertical momentum, heat and moisture fluxes at the three heights above the canopy for each stability class and for canopy without- and with leaves. Detailed results are shown in appendix 2A (see Table 2A.1). General conclusion is that under atmospheric conditions, under which the corresponding flux is dominant (e.g. heat dominates under unstable, momentum in near neutral), the vertical flux divergence is reasonably small (around $10 \%$, as assumed in the ASL). For instance, we find that over the fully-leafed canopy the average variation of $\overline{w^{\prime} u^{\prime}}$ with height is smaller than $3 \%$ compared to its value at $2.3 h_{c}$ under near-neutral conditions. For the same stability the variation of $\overline{w^{\prime} u^{\prime}}$ with height for the leafless canopy increases to about 15 $\%$ with respect to the value at $2.3 h_{c}$. Furthermore, the average variation of $\overline{w^{\prime} \theta^{\prime}}$ with height is between 10 and $20 \%$ for both canopy leaf states, compared to their values at $2.3 h_{c}$ under unstable conditions. Here we note that the sonic anemometers average over a finite volume (which means that we lose the small scale fluctuations) and that we average over 30 minutes (eliminating any larger-scale fluctuations) resulting in an observed flux which underestimates the true flux (Foken 2008b). These small- and large-scale flux losses in the observations are both height dependent, where (i) the fine-scale loss increases with increasing proximity to the surface; and (ii) the largescale loss increases with increasing distance from the surface. However, any attempt to correct for the loss of small-scale covariance always relies on an assumed shape of the co-spectrum. Inside and just above the canopy there is little we can assume. The same holds for the correction of large-scale effects. Nevertheless for the heights we have used above the canopy, a 30 minute average should suffice (see e.g. Moncrieff et al. 2005). Another possible reason for the failure 

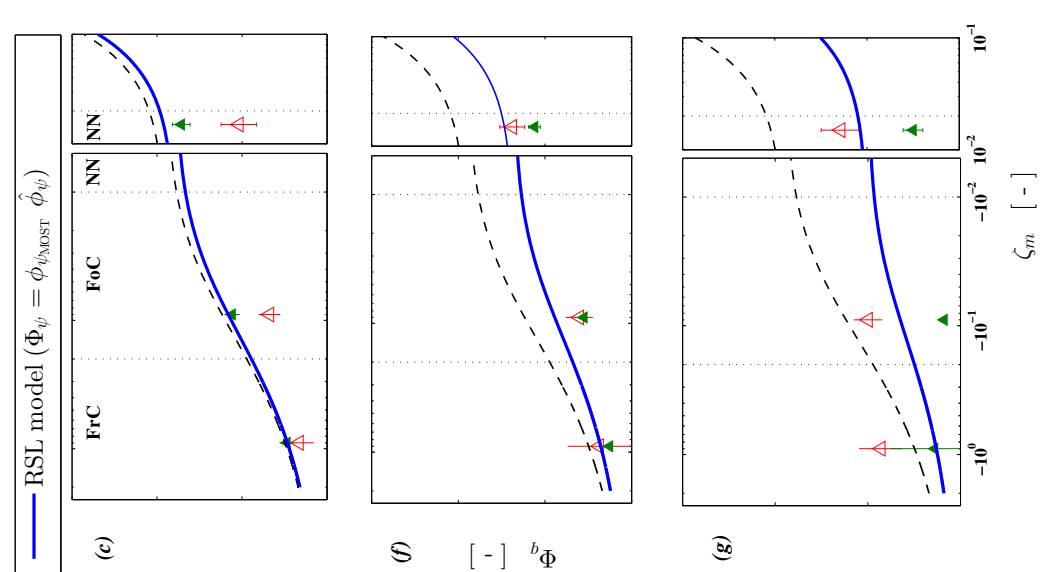

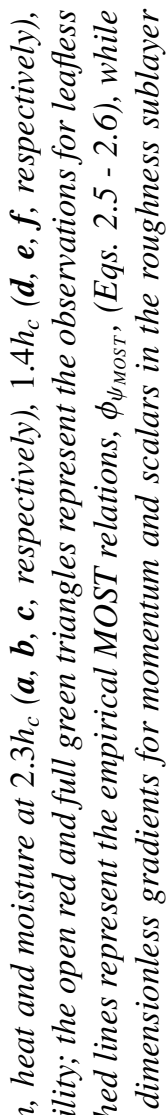
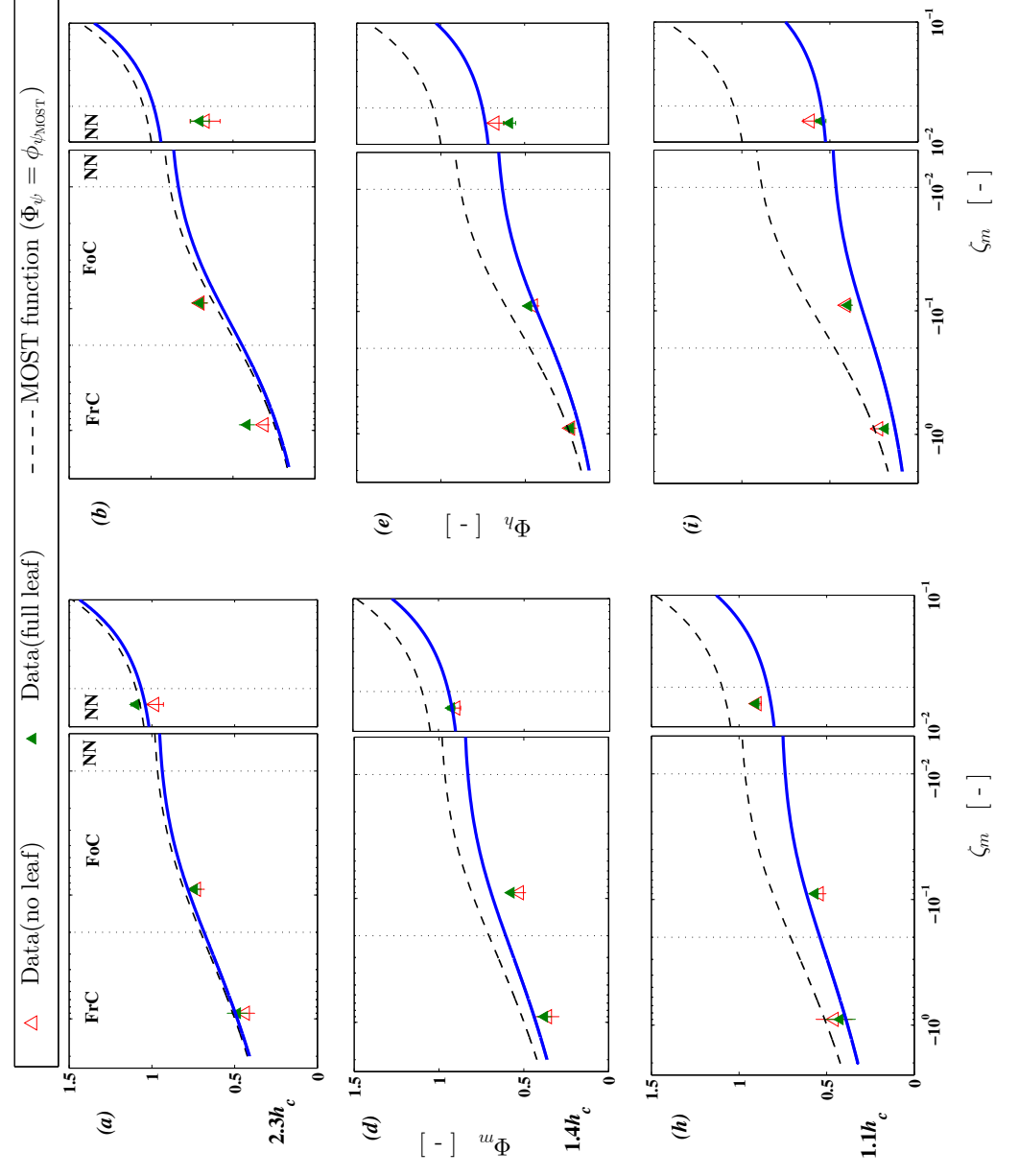

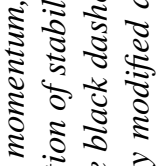

i.

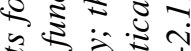

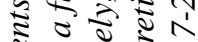

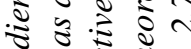

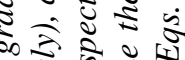

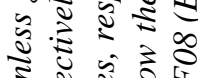

ธัँ

ปี

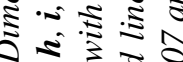

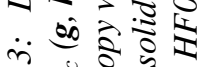

ง

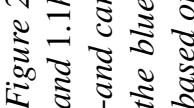


of the constant flux hypothesis is the counter-gradient phenomenon (Denmead and Bradley 1985; Denmead and Bradley 1987), which is believed to be a consequence of the non-local turbulence within and just above the canopy. We do not explicitly treat this hypothesis in our analysis, but we would like to note its possible influence on the flux divergence.

Since we observe relatively stronger vertical flux divergence for different atmospheric stabilities within the RSL (compared to that which MOST presumes), it is more appropriate to link local gradients to local fluxes. Using this approach, we quantify the deviation of the observed and modelled $\Phi_{m}, \Phi_{h}$ and $\Phi_{q}$ values from that which standard MOST would predict in the RSL (Fig. 2.3). Figure 2.3 shows that the deviation of $\Phi_{m}, \Phi_{h}$ and $\Phi_{q}$ from the standard functions (Eqs. 2.5 - 2.6) generally increases with closer proximity to the canopy top. Compared to MOST, the RSL-induced reductions of the dimensionless gradients are within the range of canopy-induced dimensionless-gradient reductions reported in the previous observational studies (Thom et al. 1975; Raupach 1979; Garratt 1980; Denmead and Bradley 1985; Högström et al. 1989; Simpson et al. 1998). At the lowest measured level $\left(1.1 h_{c}\right)$, we find a decrease in $\Phi_{m}$ of around $25 \%$ (Fig. 2.3g) and of around $55 \%$ in $\Phi_{m}$ (Fig. 2.3h). The decreases in the magnitude of and do not significantly differ across variations in canopy leaf state or stability (which results from our having used a leaf-state-dependent displacement height, see Sect. 2.4.2). The largest deviation (reduction) from MOST is recorded for $\Phi_{q}$ (around $80 \%$ ) for the fully-vegetated canopy (Fig. 2.3i). The strong reduction of $\Phi_{m}$ for the fully vegetated canopy likely results from our assumption that the displacement height for momentum and moisture are equal. We will elaborate more on this issue in the next paragraph. Similar $\Phi_{m}$ and $\Phi_{h}$ reductions are shown in Garratt (1980) at the lowest measured level above savannah under unstable conditions. Maximum reductions for $\Phi_{m}$ and $\Phi_{h}$ of up to 60 and $70 \%$, respectively, are discussed by Högström et al. (1989) at their lowest measured level $\left(1.15 h_{c}\right)$ above a pine forest under unstable conditions.

Standard MOST functions (Eqs. 2.5 -2.6) predict the observed dimensionless gradients at $2.3 h_{c}$ reasonably well (Fig. 2.3a,b,c), albeit with i) slightly higher values than the observed gradients of $\Phi_{h}$ in near-neutral conditions (Fig. 2.3b), resulting from the discontinuous character of $\Phi_{h}$ at the near-neutral limit (due to very small heat flux and temperature gradient), and ii) significantly lower observed gradients of $\Phi_{q}$ for leafless canopy (Fig. 2.3c), due to the low evapotranspiration under leafless conditions. Taking into account the standard errors of the dimensionless gradient means, dimensionless gradients predicted by MOST at the $2.3 h_{c}$ level agree well with the observations, suggesting that direct canopy influences have sufficiently diminished by this height to suggest that this level is above the RSL.

RSL-corrected MOST To complete the analysis, we evaluate HF07 and HF08's dimensionless gradient formulations that include a parameterization of the RSL's influence on MOST (Eqs. 2.7 - 2.11) above CHATS' tall deciduous canopy. The expressions presented in Eqs. $(2.7-2.11)$ capture the observed dimensionless gradients $\Phi_{m}$ and $\Phi_{h}$ very well, while they deviate for $\Phi_{q}$ 
(Fig. 2.3). Recall that the displacement height for momentum was used to calculate $\Phi_{q}$, which might lead to an underestimation of the dimensionless gradient of specific moisture at the lowest measured level. Under FoC and NN conditions for the fully leafed canopy, the displacement height evaluated as the mean height of the within-canopy moisture sources/sinks (Eq. 2.12) is around $0.53 h_{c}$, which differs significantly from the displacement height for momentum $\left(0.75 h_{c}\right)$. This discrepancy would therefore increase the calculated $\Phi_{q}$ by about $15 \%$ at the $1.1 h_{c}$ level and improve the agreement between the observations and HF08's modified $\Phi_{q}$. However, Raupach (1979) suggested that there are no acceptable physical interpretations for very low heat and moisture displacement heights and recommended using the momentum displacement height as the relevant length scale for all quantities when representing dimensionless gradients and then to "incorporate all property differences into the influence functions $\Phi_{m, h, q}$, which then depend not only upon the stability parameter but also upon dimensionless surface parameters". Based on the results presented here (especially for $\Phi_{m}$ and $\Phi_{h}$ ), we confirm that HF07 and HF08's formulation captures the primary canopy-induced effects on the turbulence and therefore recommend its use in large-scale models when parameterizing flux-gradient relationships in the vicinity of high vegetation.

There are few studies considering canopy phenology influences on flux-gradient relationships over a deciduous canopy and their variation with seasonality or diabatic stability (e.g., Simpson et al. 1998; Sakai et al. 2000). The work conducted by Simpson et al. (1998) is based on an observational analysis of fluxes and gradients of $\mathrm{CO}_{2}$ over a heterogeneous deciduous forest in Canada with average tree height of $20 \mathrm{~m}$ and average LAI of 3.6. They found approximately a 10-20\% increase in the dimensionless $\mathrm{CO}_{2}$ gradient under near-neutral conditions for pre- vs. post-senescence at the measurement level closest to canopy top $\left(1.3 h_{c}\right)$. This implies that the fully vegetated canopy is less efficient in reducing the dimensionless $\mathrm{CO}_{2}$ gradient than the canopy with less leaves, suggesting more efficient $\mathrm{CO}_{2}$ exchange for their canopy without leaves. Hence, the direction of the variation of dimensionless gradient with phenology is consistent with that found in our data set, but the magnitude differs (10-20\% in Simpson et al. (1998) versus around $2 \%$ in our data), although it should be noted that the observation heights are not exactly comparable $\left(1.3 h_{c}\right.$ vs. $\left.1.1 h_{c}\right)$. The magnitude difference of leaf-state on the dimensionless gradients most likely results from the fact that Simpson et al. (1998) neglected the seasonal variation in the displacement height when calculating the flux-gradient relationships. On the other hand, Sakai (2000) investigated the effects of canopy seasonal variation on turbulent exchange within and just above different canopy types (LAI from 1 to 5), taking phenology dependent displacement height and roughness length into account. Similar to our results, Sakai (2000, Fig. 3.4) found decreased dimensionless gradients of wind and temperature close to the canopy top $\left(1.3 h_{c}\right)$, compared to the standard MOST. However, Sakai (2000) found that due to the phenological influence on $z_{0}$ and $d$ (Fig. 3.15 in their study), the resulting drag coefficients were insensitive to the variation of the canopy leaf state under convective conditions. The latter has previously been shown for the 


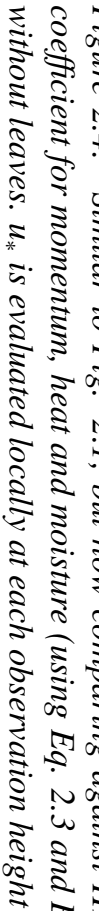
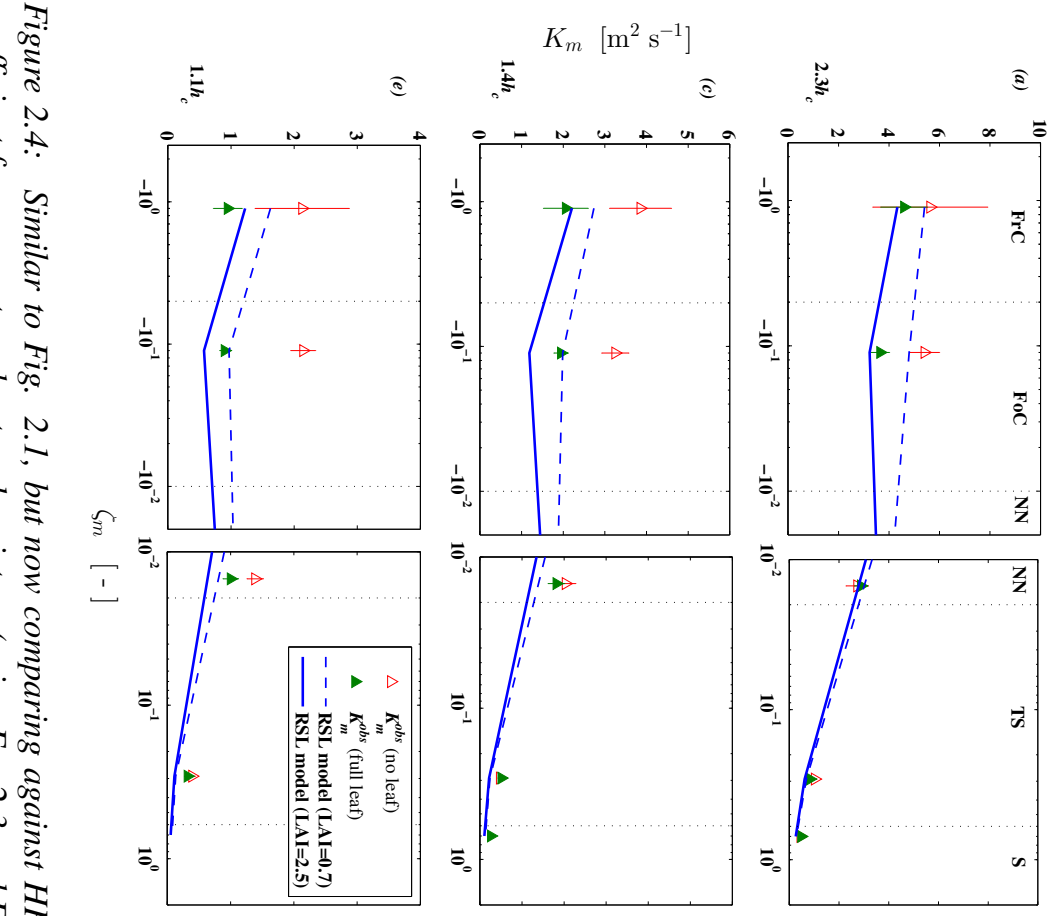

3ิ

ミ)

ร)

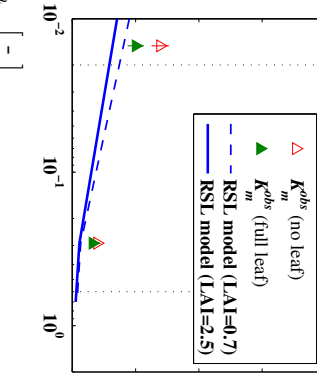

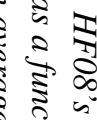

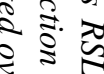

$\stackrel{2}{0}$

กิ

त

₹

ร क

है N
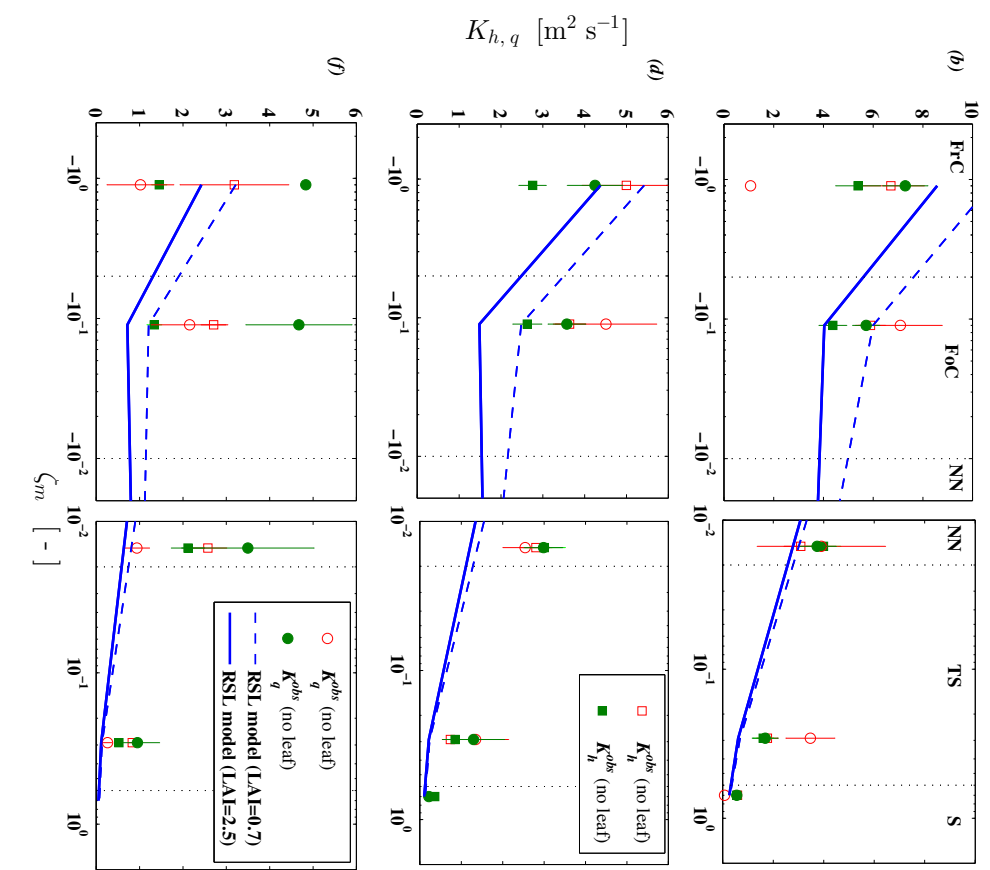

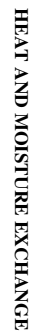


Harvard deciduous Forest (Fig. 8, Moore et al. 1996).

Our analysis in section 2.4.2 showed that the dimensionless gradients are very sensitive to the displacement height formulation, especially when evaluating dimensionless gradients near canopy top. Figure 2.4 shows a comparison between observed $K_{m}^{o b s}, K_{h}^{o b s}$ and $K_{q}^{o b s}$ (Fig. 2.1) and the $K_{m}, K_{h}$ and $K_{q}$ parameterized using the observed local friction velocity and HF07 and HF08's RSL modification of MOST (Eqs. $2.7-2.11$ ), which we have applied as it would be if it were implemented within a large-scale atmospheric models where canopy characteristics are only available via satellite (see section 2.2.1). Figure 2.4 further indicates that phenology has a large influence on $K_{m}, K_{h}$ and $K_{q}$. Moreover, the HF07 and HF08 RSL parameterization shows satisfactory agreement with the CHATS observed $K_{m}, K_{h}$ and $K_{q}$, which confirms their applicability for parameterizing canopy-atmosphere turbulent exchange over tall vegetation in weather and climate models for this range of atmospheric stability. However, we should note that the agreement of the RSL model with the observations becomes weaker under stronger instability (shear-free conditions). Under these conditions the effect of the larger-scale convective eddies on the turbulent transport in the RSL can be a relevant factor (Fitzjarrald et al. 1988; Zilitinkevich et al. 2006). We note that the RSL model does not physically accounts for these large convective eddies.

Furthermore, the $K_{q}$ is similar to $K_{h}$ only at the level $2.3 h_{c}$ and $1.4 h_{c}$. Therefore, at this height, the assumption for the equality of the universal dimensionless functions between heat and moisture (Eq. 2.6) still holds. However, at the level $1.1 h_{c}$ the differences were larger, where $K_{q}$ is larger than $K_{h}$ for the full-leaf canopy. We note that these differences can be a consequence of different partitioning of the specific humidity. Namely, the $K_{q}$ and $K_{h}$ are influenced not only by the plant transpiration, but also by soil evaporation (Dupont and Patton 2012). Since during the CHATS experiment there were days with irrigation (Patton et al. 2011), it can potentially influence the specific moisture partitioning and its exchange close to canopy top.

Given that the vertical turbulent fluxes are reasonably constant with height, our findings confirm that the observed reductions in $\Phi_{m}, \Phi_{h}$ and $\Phi_{q}$ in the RSL largely result from smaller mean wind speed and scalar gradients with increasing proximity to canopy top (see Dupont andPatton 2012b) compared to those predicted by MOST. This decrease in mean gradients near the canopyatmosphere interface is related to the turbulent coherent structures formed in this layer (Raupach et al. 1996; Finnigan et al. 2009). Parameterization schemes generally characterize the coherent structures using a mixing length, which for surface-layer scaling is $k\left(z-d_{\psi}\right)$ according to Prandtl's mixed layer theory. To bring canopy influences into their RSL parameterization, HF07 and FH08 incorporate an additional length scale associated with the coherent structures generated via the shear-instability at canopy top: the vorticity thickness, $\delta_{\omega}$, which is defined as $\bar{u} /[d \bar{u} / d z]$ at canopy top and therefore equal to $l / \beta$ (HF07). Given the importance of $\delta_{\omega}$ in HF07's and HF08's RSL parameterization, we will elaborate on the impact of phenology and stability on the turbulent exchange coefficients discussed in section 4.1. For that, we extend the analysis conducted 


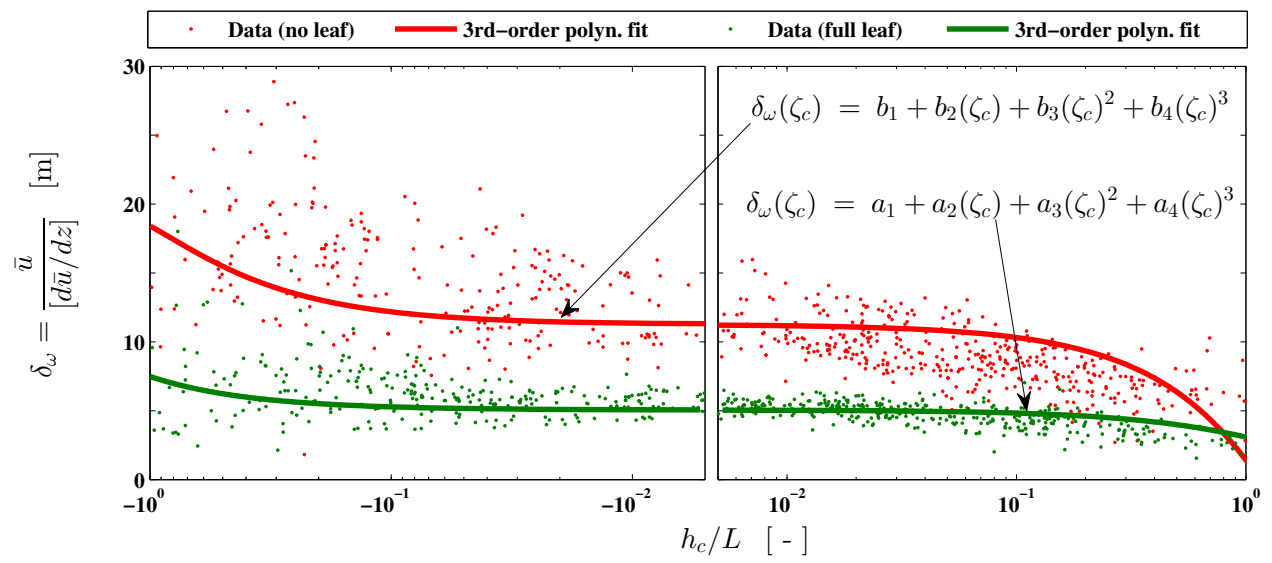

Figure 2.5: Observed vorticity thickness, $\delta_{\omega}$, calculated from the measured mean wind speed and gradients at the canopy top, as a function of the stability at the same level before and after the leaf-out. The red and the green solid lines represent the best cubic polynomial fit for each canopy leaf state, respectively, with the following constants: $a_{1}=10.10, a_{2}=-4.25, a_{3}=0.46$, $a_{4}=0.12$ and $b_{1}=22.52, b_{2}=-18.66, b_{3}=-2.70, b_{4}=1.68$.

by Dupont and Patton (2012a; 2012b) by calculating the vorticity thickness as a function of the stability at CHATS.

Figure 2.5 confirms that $\delta_{\omega}$ at the canopy top strongly depends on both canopy phenology and diabatic stability. The larger $\delta_{\omega}$ for the leafless canopy indicates the presence of larger scales in the turbulence, which explains the larger exchange coefficients for the leafless canopy discussed in Sect. 2.4.1.

\section{The role of phenology on $K$ - parameterization based on TKE and dissipation}

In order to determine parameterized turbulent diffusivities according to Eq. 2.14 and Eq. 2.15, both TKE and dissipation need to be determined from the CHATS observations. TKE is derived from the variances for the three wind speed components, where the latter have been extensively discussed by Dupont and Patton (2012a; 2012b). Dissipation is derived using the Fourier spectrum of the horizontal streamwise wind speed component, $S_{u}(k)$, where the energy density is assumed to follow:

$$
S_{u}(k)=\alpha \varepsilon^{2 / 3} k^{-5 / 3} .
$$

Details of the method used here to calculate dissipation are presented in appendix 2B. Figure 2.6 shows that the $K$-parameterizations based on Eq. 2.14 and Eq. 2.15 depend on the phenological evolution under unstable and near-neutral conditions. While for near-neutral conditions both methods give very similar results compared to the observed $K_{m}^{o b s}$ (both showing an increase of $K$ 

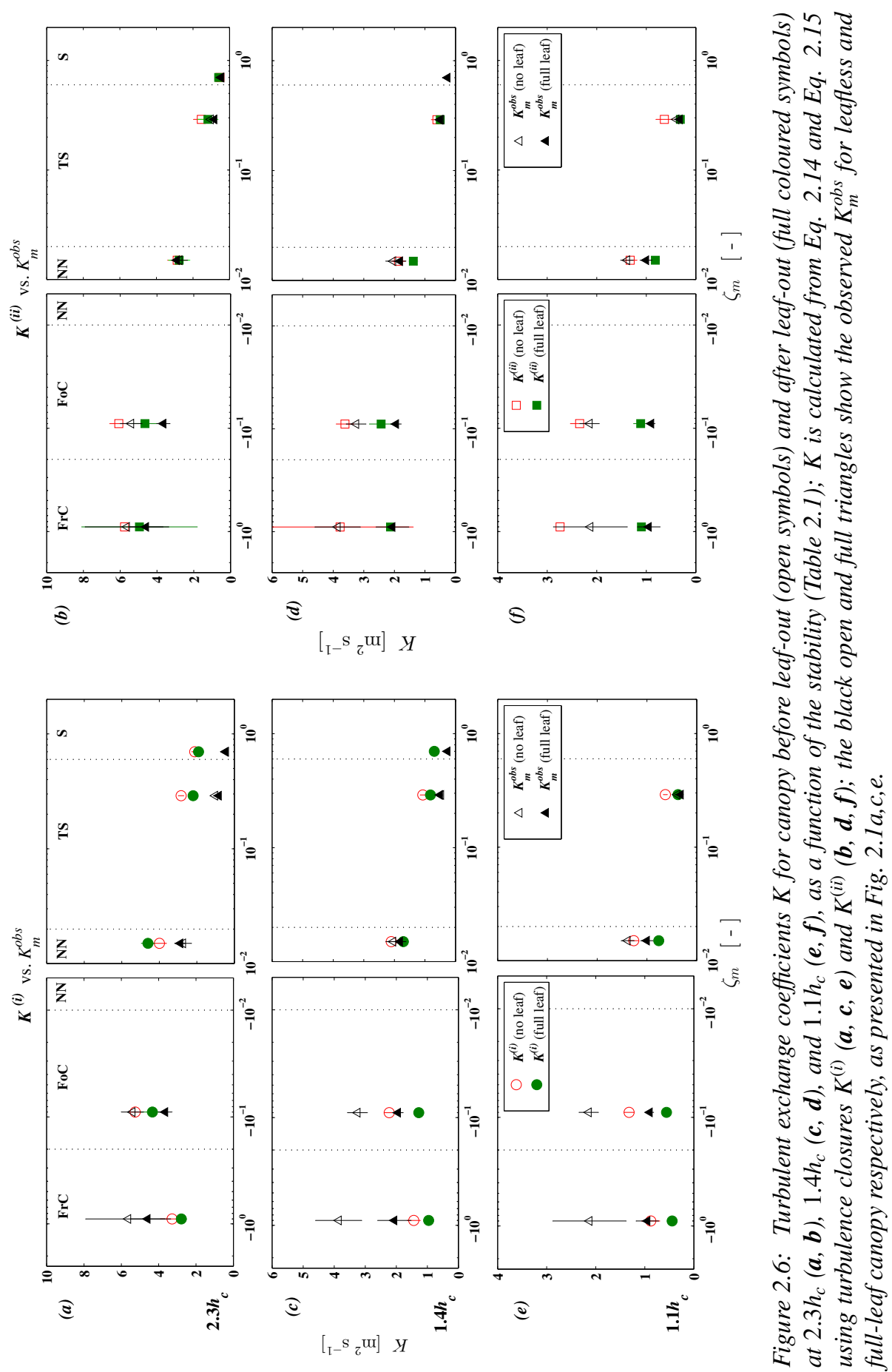
from about $1 \mathrm{~ms}^{-1}$ at $z=1.1 h_{c}$ to $3.5 \mathrm{~ms}^{-1}$ at $z=2.3 h_{c}$ ), they differ in magnitude for unstable conditions. For instance, at $1.1 h_{c}$ for FrC and FoC stability classes $K^{i i}$ is larger than the $K^{i}$, but $K^{i}$ is smaller than $K_{m}^{o b s}$ (Fig. 2.6e). Furthermore, both methods show the correct variation with height, but $K^{i}$ fails to approach the observed $K_{m}^{o b s}$ values under unstable conditions a result which suggests that the constant $c_{\mu}=0.03$ used in $K^{i}$ is not applicable in this dataset under unstable conditions (although, consistent with Katul et al (2004) analysis, this $c_{\mu}$ value works well for near neutral data).

The turbulence closures $K^{i}$ and $K^{i i}$ are similar under near-neutral conditions (Katul et al. 2004). Katul et al. (2004) presented a detailed analysis of the similarities between both of these turbulence closures applied to neutrally stratified, homogeneous, steady-state flow, within and just above canopies with different morphology (i.e. rice, corn, pine trees, etc). Distinct in our $K^{i}$ analysis is the application of a phenology and diabatic-stability varying displacement height in defining the turbulent length scale. Using this evolving $d_{m}$ in $K^{i}$ (Fig. 2.6) yields a $15 \%$ increase in $K^{i}$ compared to when $d_{f i x}$ is used (not shown), which implies that canopy phenology effects on $K$-parameterization were largely captured within $K_{f i x}$, since the turbulent kinetic energy does not show significant phenology dependence (Fig. 2.7a). We can therefore conclude that closures based on dimensionless gradients (Eq. 2.2) and TKE (Eq. 2.14) need to include a turbulent length scale which accounts for canopy phenology and atmospheric stability changes.

The $K^{i i}$ formulation (Eq. 2.15) offers a distinct advantage by not requiring an explicit length scale. The information for the turbulence variation is captured in the ratio between the $e^{2}$ and the TKE dissipation $\varepsilon$ (Eq. 2.15). Figure 2.6 indicates that the method better reproduces the observed exchange coefficient than does the $K^{(i)}$ method. To further discuss the reasons for this differences in the parameterization methods, we extend our analysis to investigate the influence of canopy phenology changes on $\varepsilon$ and additionally on turbulent transport of TKE.

Assuming stationary, near neutral and horizontal homogeneous conditions, the TKE budget in the RSL above the canopy reads (Brunet et al. 1994):

$$
\frac{\partial e}{\partial t}=0=\underbrace{-\overline{w^{\prime} u^{\prime}} \frac{\partial \bar{u}}{\partial z}}_{P_{s}}-\underbrace{\frac{\partial \overline{w^{\prime} e}}{\partial z}}_{T_{t}}-\underbrace{\frac{1}{\rho} \frac{\partial \overline{p^{\prime} w^{\prime}}}{\partial z}}_{T_{p}}-\varepsilon,
$$

which is a balance between shear production $\left(P_{s}\right)$, turbulent transport $\left(T_{t}\right)$, and pressure transport $\left(T_{p}\right)$, and dissipation $(\varepsilon)$. The CHATS data enables direct calculation of $P_{s}, T_{t}$ and $\varepsilon$. Due to the difficulty in measuring the pressure transport term, we represent this term as a residual TKE (R).

Figure 2.7c shows $K^{(i i)}$ (similar to Fig. 2.6b,d,f), but now focuses on near-neutral conditions as a function of height (Fig. 2.7c). To support the analysis, we also show the total $e$ (Fig. 2.7a), and the TKE shear production $\left(P_{s}\right)$ and the dissipation $(\varepsilon)$ (Fig. 2.7b). All quantities in Fig. 2.7 are presented in dimensional form to enable direct comparison between dimensional exchange coefficient and its determining factors. We observe that $K^{(i i)}$-values (Fig 2.7c) are larger under 

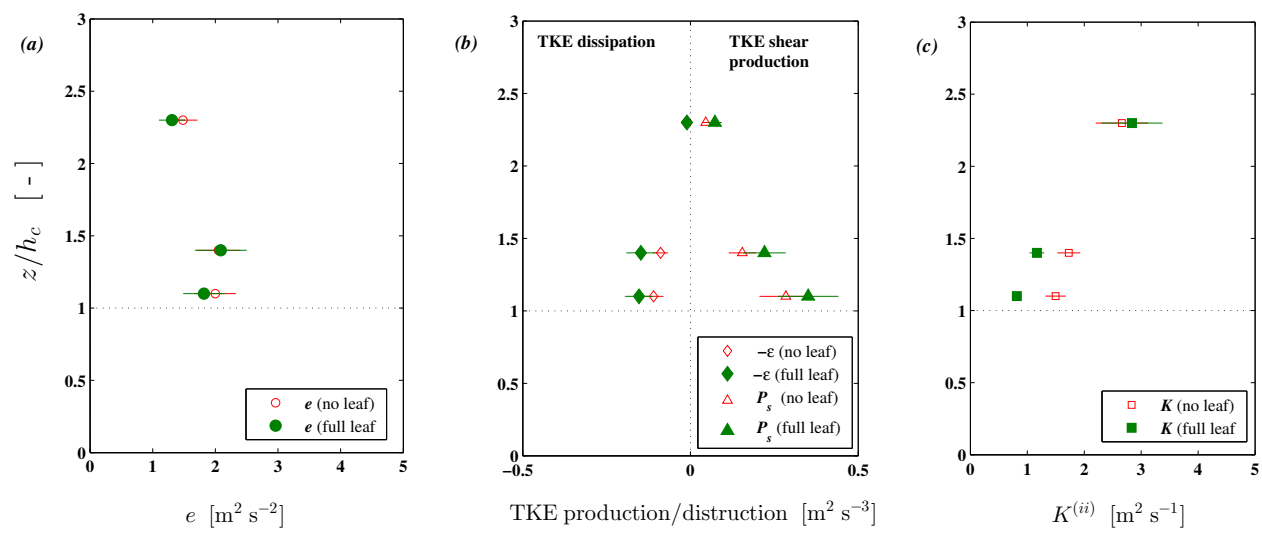

Figure 2.7: (a) Turbulent kinetic energy $(e),(b)$ TKE shear production $\left(P_{s}\right)$ and TKE dissipation $(\varepsilon)$, and $(c) K^{i i}$ (Eq. 2.15), calculated from CHATS observations at different heights above the canopy under near-neutral conditions (Table 2.1).

leafless conditions in spite of the fact that $e$ has similar values under leaf and non-leaf conditions, which likely arises because the fine-scale boundary layers produced on the leaves increase dissipation's dependence on leaf state.

Compared to upper levels, shear production of TKE is largest near canopy top (Fig. 2.7b). At all levels the shear-production of TKE is larger for the fully-leafed canopy than for leafless canopy, due to the larger mean velocity gradient for full-leaf conditions (Dupont and Patton 2012b). Under near-neutral conditions, the vertical variation of TKE dissipation and its evolution with leaf-state generally follows TKE shear production (Fig. 2.7b), while turbulent transport of TKE does not significantly change with leaf state (Fig. 2.8a). Apparently larger production and dissipation lead to only slightly more TKE (Fig. 2.7a). Therefore, the evolution of $K^{(i i)}$ with leaf state must result from the leaf-induced increased dissipation.

To conclude, we find that canopy phenology influences the production and dissipation of TKE. There is no significant phenological influence on the above- canopy turbulent transport of TKE or residual TKE (Fig. 2.8a and 2.8b, respectively). The larger TKE shear production for full-leaf canopy is related to the canopy-induced turbulence (Raupach et al. 1996; Finnigan et al. 2009; Patton and Finnigan 2013). However, our observational analysis indicates that under leafless canopy conditions, enhanced coupling between the atmosphere and the ground surface results in more effective mixing (quantified by the larger values, Fig. 2.7c). A plausible explanation for this result is related to the combined effects of large-scale surface-layer eddies and coherent eddies formed via the inflection-point instability induced at canopy top (Dupont and Patton 2012a; 2012b). While denser canopies induce more shear at canopy top, sparser canopies allow the canopy-top shear-induced eddies to penetrate deeper into the canopy which more efficiently mixes 

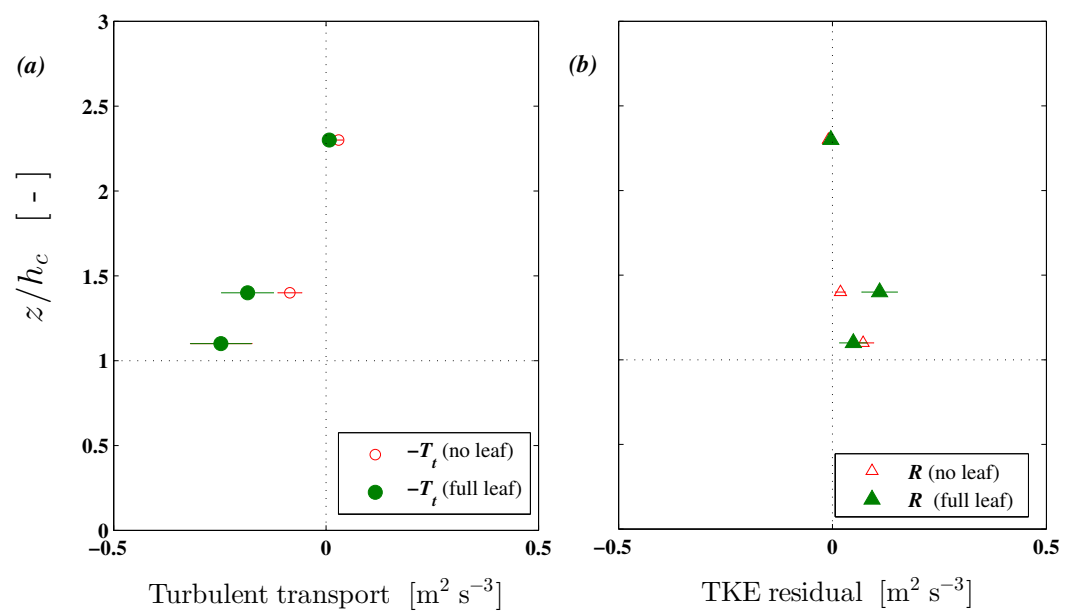

Figure 2.8: (a) Turbulent transport of TKE and (b) residual TKE, calculated from CHATS observations at different heights above the canopy under near-neutral conditions (Eq. 2.15 and Table 2.1).

the layers within and above the canopies (as characterized by the increased vorticity thickness, Fig. 2.5).

\subsection{Conclusions}

The effect of canopy phenology on turbulence within the roughness sublayer above a tall deciduous walnut orchard is studied as a function of atmospheric stratification. The validity of different turbulence closure formulations for the turbulent exchange coefficient is investigated and compared with the turbulent exchange coefficient calculated from observed vertical fluxes and mean gradients. To this end, we analyzed a complete data set of observations gathered during a whole season at the Canopy Horizontal Array Turbulence Study (CHATS) experiment. Based on our findings, we reached the following conclusions:

- Turbulent structure above tall deciduous vegetation depends strongly on canopy phenology changes, as quantified by the turbulent exchange coefficient. Leafless canopy conditions increases the turbulent exchange coefficient of momentum, heat and moisture under both unstable and near-neutral conditions due to better atmosphere-ground surface coupling.

- Except for the turbulent closure $K^{(i)}$, under unstable conditions, the turbulent closure formulations for the turbulent exchange coefficient are able to reproduce the observations relatively well when using a canopy phenology and diabatic stability dependent displacement 
height in the turbulent length scale. Parameterizations based on Eq. 2.14 and Eq. 2.15 show the correct height dependence above the canopy. They also satisfactorily reproduce the leaf-state dependence. Parameterizations based on Eq. 2.2 using modified dimensionless gradient to account for the RSL effects satisfactorily represent the observations in terms of both their height dependence and their phenology dependence.

- A length scale (displacement height or vorticity thickness) formulation which accounts for the canopy leaf state and diabatic stability is crucial to accurately representing flux-gradient relationships of state variables within the roughness sublayer under the seasonal evolution of canopy foliage; a relevant finding for roughness sublayer turbulence parameterization.

- Harman and Finnigan $(2007 ; 2008)$ roughness sublayer parameterization of canopy induced modification to flux-gradient relationships are evaluated and are in satisfactory agreement with the CHATS observations. We therefore recommend applying their parameterization when attempting to represent turbulent fluxes via turbulent exchange coefficients.

- Under near-neutral conditions, TKE dissipation generally follows TKE shear production for both canopy leaf states, while turbulent transport of TKE shows no significant dependence on the leaf state. 
Table 2A.1: Ratio between the vertical momentum, heat and moisture fluxes at different heights above the canopy relative to the highest measured level $(23 \mathrm{~m})$ for both leaf states (no leaf, full leaf) and different stability class (Table 2.1).

\begin{tabular}{|c|c|c|c|c|}
\hline Stability & \multicolumn{2}{|c|}{ No leaf } & \multicolumn{2}{|c|}{ Ful leaf } \\
\hline & $\overline{w^{\prime} u^{\prime}}{ }_{23} / \overline{w^{\prime} u^{\prime}}{ }_{14}$ & $\overline{w^{\prime} u^{\prime}}{ }_{23} /{\overline{w^{\prime} u^{\prime}}}_{11}$ & $\overline{w^{\prime} u^{\prime}}{ }_{23} / \overline{w^{\prime} u^{\prime}}{ }_{14}$ & $\overline{w^{\prime} u^{\prime}}{ }_{23} /{\overline{w^{\prime} u^{\prime}}}_{11}$ \\
\hline FrC & 1.16 & 1.30 & 1.16 & 1.58 \\
\hline FoC & 1.13 & 1.21 & 1.21 & 1.43 \\
\hline NN & 0.83 & 0.85 & 0.97 & 1.00 \\
\hline TS & 1.14 & 1.43 & 1.11 & 1.61 \\
\hline$S$ & 1.00 & $\mathrm{n} / \mathrm{a}$ & 1.17 & 1.63 \\
\hline & $\overline{w^{\prime} \theta^{\prime}}{ }_{23} / \overline{w^{\prime} \theta^{\prime}}{ }_{14}$ & $\overline{w^{\prime} \theta^{\prime}}{ }_{23} / \overline{w^{\prime} \theta^{\prime}}{ }_{11}$ & $\overline{w^{\prime} \theta^{\prime}}{ }_{23} / \overline{w^{\prime} \theta^{\prime}}{ }_{14}$ & $\overline{w^{\prime} \theta^{\prime}}{ }_{23} / \overline{w^{\prime} \theta^{\prime}}{ }_{11}$ \\
\hline FrC & 0.85 & 0.80 & 0.82 & 0.89 \\
\hline FoC & 0.86 & 0.80 & 0.96 & 0.92 \\
\hline $\mathrm{NN}$ & 1.73 & 3.07 & 1.12 & 1.35 \\
\hline $\mathrm{TS}$ & 0.88 & 0.99 & 0.82 & 0.91 \\
\hline S & 0.73 & $\mathrm{n} / \mathrm{a}$ & 1.95 & 1.13 \\
\hline & & & ${\overline{w^{\prime} q^{\prime}}}_{23} /{\overline{w^{\prime} q^{\prime}}}_{14}$ & $\overline{w^{\prime} q^{\prime}}{ }_{23} /{\overline{w^{\prime} q^{\prime}}}_{11}$ \\
\hline FrC & & & 0.97 & 1.13 \\
\hline FoC & & & 1.01 & 1.06 \\
\hline $\mathrm{NN}$ & & & 0.11 & 1.13 \\
\hline TS & & & 1.09 & 1.35 \\
\hline S & & & 2.79 & 2.78 \\
\hline
\end{tabular}

\section{A Appendix: Analysis of the vertical flux divergence}

\section{B Appendix: Steps to calculate TKE dissipation from the CHATS data}

The dissipation is derived from the Fourier spectrum of the horizontal streamwise wind speed component, $S_{u}(k)$, where the energy density follows the expression: $S_{u}(k)=\alpha \varepsilon^{2 / 3} k^{-5 / 3}$. To evaluate $\varepsilon$, the following steps are made for each 30 minute interval of $60 \mathrm{~Hz}$ raw data:

i) the spectrum $S_{u}(k)$ is smoothed over 100 spectral points;

ii) $\varepsilon$ is determined from the equation of $S_{u}(k)$ for each wavenumber in the spectrum;

iii) using a moving window covering $20 \%$ of the spectrum (moving by one wave number step at a time) the coefficient of variation $\left(\sigma_{\varepsilon} / \bar{\varepsilon}\right)$ as well as the slope of the spectrum is determined for each window position;

iv) if, for a given window position, the coefficient of variation of epsilon is below $30 \%$ and 
the slope of the spectrum is within $30 \%$ of $-5 / 3$ the mean value of epsilon for that window position is considered to be valid;

v) the $\varepsilon$ value for the entire 30 minute interval is determined as the mean of all valid values of $\varepsilon$ found in the above procedure. 


\title{
Integrating canopy and large-scale effects in the convective boundary-layer dynamics during CHATS experiment
}

\begin{abstract}
By characterizing the dynamics of a convective boundary layer above a relatively sparse and uniform orchard canopy, we investigated the impact of the roughness sublayer (RSL) representation on the predicted diurnal variability of surface fluxes and state variables. Our approach combined numerical experiments, using an atmospheric mixed-layer model including a land surface-vegetation representation, and measurements from the Canopy Horizontal Array Turbulence Study (CHATS) field experiment near Dixon, California. The RSL is parameterized using an additional factor in the standard Monin-Obukhov Similarity Theory fux-profile relationships that takes into account the canopy influence on the atmospheric flow. We selected a representative case characterised by southerly wind conditions to ensure well-developed RSL over the orchard canopy. We then investigated the sensitivity of the diurnal variability of the boundary-layer dynamics to the changes in the RSL key scales, the canopy adjustment length scale, $L_{c}$, and the $\beta=u_{*} /|U|$ ratio at the top of the canopy, due to their stability and dependence on canopy structure. We found that the inclusion of the RSL parameterisation resulted in improved prediction of the diurnal evolution of the near-surface mean quantities (e.g. up to
\end{abstract}

This chapter is published as Shapkalijevski et al. (2017), with Appendix 3C as a supplementary material. 
$50 \%$ for the wind velocity) and transfer (drag) coefficients. We found relatively insignificant effects on the modelled surface fluxes (e.g. up to $5 \%$ for the friction velocity, while $3 \%$ for the sensible and latent heat), which is due to the compensating effect between the mean gradients and the drag coefficients, which are both largely affected by the RSL parameterisation. When varying $L_{c}$ (from 10 to $20 \mathrm{~m}$ ) and $\beta$ (from 0.25 to 0.4 ), based on observational evidence, the predicted friction velocity is found to vary by up to $25 \%$ and the modelled surface energy fluxes (SH and LE) vary up to $2 \%$ and $9 \%$, respectively. Consequently, the boundary-layer height varies up to $6 \%$. Furthermore, our analysis indicated that to interpret the CHATS measurements above the canopy, the contributions of non-local effects such as entrainment, subsidence and the advection of heat and moisture over the CHATS site need to be taken into account.

\subsection{Introduction}

The atmospheric boundary layer (ABL), as a component of the global climate system, is characterized by the turbulent exchange of energy, momentum and matter between the Earth's surface and the lower atmosphere, as well as by the influence of larger-scale atmospheric processes (Stull 1988). Tall plant canopies modify turbulence at the canopy-atmosphere interface, leading to specific turbulent organised structures (Raupach 1998). These coherent turbulent structures in the canopy vicinity are similar in nature to eddies developed in a plane mixing layer (Raupach1996; Finnigan 2000; Finnigan et al. 2009). The layer in which these turbulent structures appear and affect the atmospheric flow is called the roughness sublayer (RSL). These structures are responsible for majority of the momentum and turbulent kinetic energy exchange between canopy and atmosphere Finnigan 2000; Finnigan et al. 2009). Dependent on canopy density and height, as well as atmospheric diabatic stability, the vertical extent of the RSL is estimated to reach up to 2-3 canopy heights (Dupont and Patton 2012a; Shapkalijevski et al. 2016). Representing the ABL dynamics, considering the RSL turbulence within the system, may be of importance in numerical weather prediction models (NWP) (Physick and Garratt 1995; Harman 2012).

A number of observational studies have demonstrated the importance of canopy effects on the turbulent exchange of energy, mass and momentum within the RSL for different canopy types (e.g. Thom et al. 1975; Raupach 1979; Denmead and Bradley 1985, Högström1989). They all pointed out the failure of the traditional Monin-Obukhov similarity theory (MOST, Monin and Obukhov 1954) to link turbulent fluxes to the mean profiles within the RSL. To account for the canopy effects, a number of different formulations parameterizing the effect of RSL have been proposed to modify the standard MOST flux-profile relationships (Garratt 1980 Cellier and Brunet 1992; Raupach 1992,Mölder1999; Harman and Finnigan 2007; 2008; De Ridder 2010). The latter resulted in improved flux calculations just above the canopy when inferred from profile measurements (Mölder1999; De Ridder 2010).

The flux-profile relationships are commonly used within the surface scheme of the atmo- 
spheric models. There have been efforts to incorporate the effect of RSL turbulence, by using the above-mentioned RSL-adapted flux-profile relationship, in the surface schemes of numerical atmospheric models (Physick and Garratt 1995; Harman 2012). Physick and Garratt (1995), who incorporated a relatively simple RSL parameterization within the surface scheme of a mesoscale model, studied the impact of the RSL on the deposition velocity and mean variables above the canopy. Physick and Garratt (1995) found significant variation in mean wind speed within the RSL, while only small (less than $3 \%$ ) on surface fluxes. Harman (2012) later implemented a more physically sound RSL formulation (based on Harman and Finnigan 2007; 2008) in the surface-energy balance (SEB) of a one-dimensional single column atmospheric model, in order to study the effect of the RSL on the coupling between a canopy and the boundary layer. Based on their (Harman and Finnigan 2007; 2008) RSL formulation, the roughness parameters (e.g. the roughness length of momentum and scalars, displacement plane) are stability dependent variables. Harman (2012) found an altered surface fluxes about $25 \%$ (e.g. sensible heat flux and the friction velocity), and also effects on mean boundary state variables (e.g. wind speed, potential temperature) just above the canopy when RSL is applied.

Extending these previous works, our study aimed to elucidate the ABL system for real conditions, taking the representation of the RSL into account. In order to consider all the relevant physical processes needed to represent the diurnal variability of the state variables above the canopy, we implemented the RSL formulation proposed by Harman and Finnigan $(2007 ; 2008)$ and embedded it in a coupled soil-vegetation-atmosphere mixed-layer model (van Heerwaarden 2009). The model has been successfully employed in a number of studies based on field observations gathered above low vegetation (e.g. van Heerwaarden 2009) or influenced by complex surface heterogeneity and topography (e.g. Pietersen et al. 2015). Here, we extend its applicability, by employing the RSL model (Harman and Finnigan 2007; 2008) to study a surface with relatively tall and sparse uniform plant canopy. In order to constrain and evaluate our numerical experiments, we took advantage of the comprehensive data set-gathered during the Canopy Horizontal Array Turbulence Study (CHATS) experiment (Patton et al. 2011), paying special attention to sensitivity analysis of the CBL dynamics to the scaling variables that govern the RSL parameterization. We focused on the sensitivity of the model results to changes in the canopy adjustment length scale, $L_{c}$, and the $\beta=u_{*} /|U|$ ratio at the canopy top, which are dependent on respectively the canopy structure and atmospheric stability.

Our research is thus an exploratory study of the potential alterations to the boundary-layer dynamics as calculated by large-scale models (Chen and Dudhia 2001), when the RSL is taken into account. 


\subsection{Methods}

\subsubsection{CHATS data}

The CHATS experiment took place in the spring of 2007 in one of Cilker Orchard's walnut blocks in Dixon, California, USA. A detailed description of the site, instrumentation and data treatment has been provided by Patton et al. (2011) and Dupont and Patton (2012a). Here we focus on the specific observations used in this study and on the criteria used to select the representative cases.

The observations analysed in this study were made on a $30 \mathrm{~m}$ mast located near the northernmost border of the orchard site in order to ensure a fetch of about $1.5 \mathrm{~km}$ for the predominant southerly winds (see Fig. 1a and Fig. 3 in Dupont and Patton 2012a). The average height of the trees $\left(h_{c}\right)$ was estimated to be $10 \mathrm{~m}$. Wind, temperature and specific humidity were measured at 13 levels on the mast (see Patton 2011). The shortwave and longwave radiation above the canopy were measured at $6 \mathrm{~m}$ above the canopy top. The soil properties were measured at a depth of 0.05 m. The NCAR Raman-shifted Eye-safe Aerosol Lidar (REAL) monitored reflectivity in order to evaluate the evolution of the boundary-layer height, $h$ (Patton 2011). The Lidar measurements enabled us to retrieve the evolution of $h$ from the aerosol backscatter signal (see supplementary material for the method and the data treatment procedures). The leaf area index (LAI) was also measured before and after the growing (leaf-out) season (Patton 2011). Although the LAI varied from 0.7 to $2.5 \mathrm{~m}^{2}$ (leaf area) $\mathrm{m}^{-2}$ (surface area) depending on the seasonality (before and after leaf-out, respectively), we took the value of 2.5 for the LAI to represent a fully vegetated canopy. It is important to note that due to the sparseness of the orchard canopy the insolation at the ground was relatively high, leading to high available energy at the soil. In consequence, the soil-related fluxes of sensible and latent heat were relatively important for the turbulent exchange processes within and above the canopy (Dupont and Patton 2012b; Shapkalijevski et al. 2016).

The CHATS dataset is used in our study to initialise and constrain our soil-vegetation and atmosphere modelling system. The model evaluation of the diurnal variability of the state variables in and above the roughness sublayer makes use of diurnal observations of the mean and turbulent variables at the same heights (at the canopy top $(10 \mathrm{~m})$ and at $19 \mathrm{~m}$ above the canopy) as for the selected study cases (section 3.2.3).

\subsubsection{Soil-vegetation-atmosphere model}

An atmospheric boundary-layer model with a zero-order jump approach, based on mixed-layer theory (Lilly 1968; Tennekes and Driedonks 1981; Vilà-Guerau de Arellano et al. 2015), was used to calculate the evolution of the well-mixed (slab) state variables and the evolution of boundary layer height. It is based on the vertical integration of the slab-averaged governing equations of thermodynamic variables and atmospheric constituents well above the canopy. At the upper boundary of the atmospheric model, the thermal inversion layer separates the well-mixed layer 


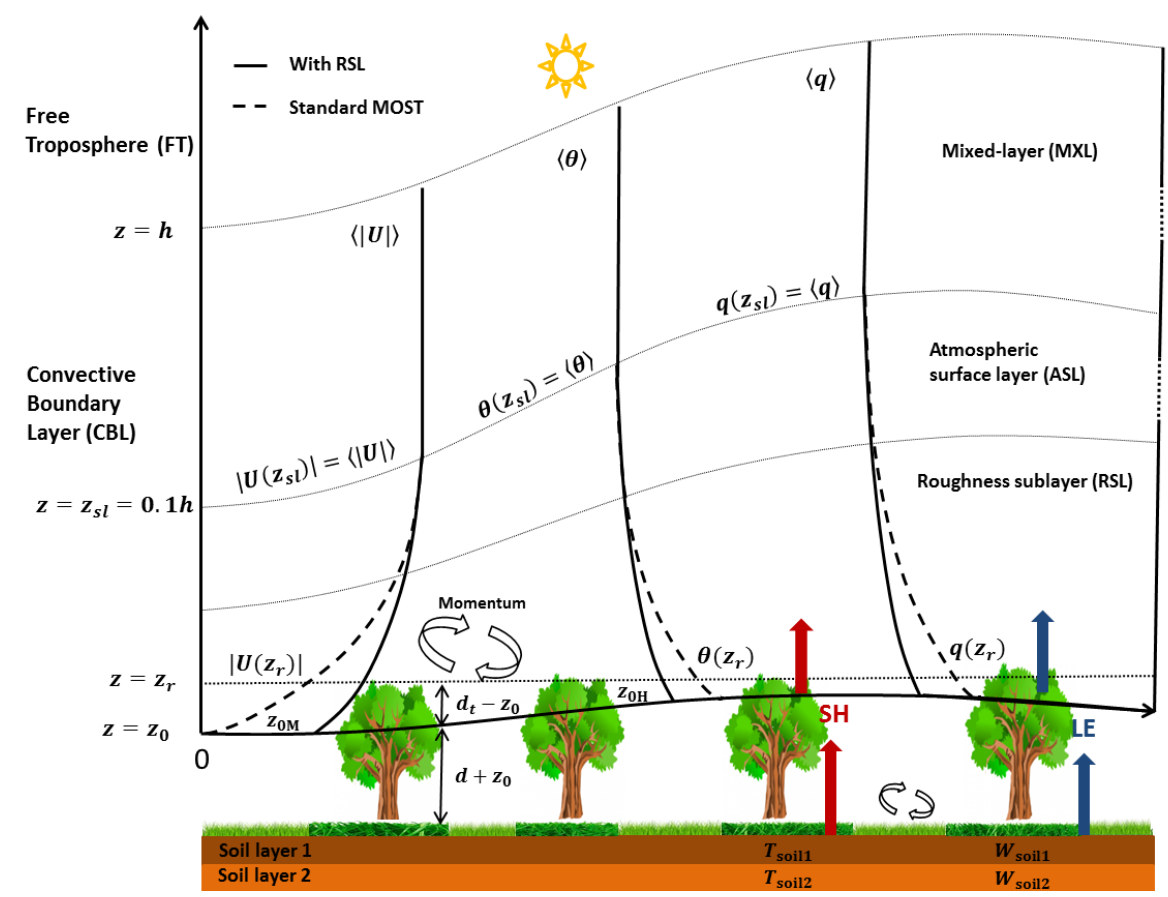

Figure 3.1: Schematic overview of the coupled land-vegetation-atmospheric system and its representation in the mixed-layer model. The vertical origin of the co-ordinate system is placed at the displacement height d. The height of the surface layer is estimated as $10 \%$ of the boundary-layer height (Stull 1988). The scheme illustrates the diurnal (convective) evolution of the boundarylayer height $(h)$ and stability dependent roughness lengths for momentum and scalars $\left(z_{0 M}\right.$ and $z_{0 H}$ ). Profiles of boundary-layer state variables (wind speed, $|U|$, potential temperature, $<\theta>$, and specific humidity, $\langle q>$ ), are also presented, both including and omitting the RSL effects in the flux-gradient relationships.

(MXL) from the free troposphere (FT). This separation is represented by a finite jump in the constituent under consideration (FT values minus MXL value) over an infinitesimal depth. At the bottom, we included a representation of the surface roughness sublayer (RSL), which is characterized by steep mean gradients, connecting the surface to the lower part of the surface layer (ASL). The ASL then connects the RSL to the MXL (Fig. 3.1). The predicted boundary-layer state variables (wind speed, potential temperature and specific humidity) and the boundary-layer height $(h)$ by the model are presented later in this section.

Based on the mixed-layer model, the diurnal variability of the mean thermodynamic variables 
and atmospheric constituents reads as follows:

$$
\frac{d<\varphi>}{d t}=\frac{\left(\overline{w^{\prime} \varphi^{\prime}}\right)_{s}-\left(\overline{w^{\prime} \varphi^{\prime}}\right)_{e}}{h}+A d v_{\varphi}
$$

where $\left(\overline{w^{\prime} \varphi^{\prime}}\right)_{s}$ and $\left(\overline{w^{\prime} \varphi^{\prime}}\right)_{e}$ are the vertical turbulent kinematic fluxes of a certain variable $\varphi$ $(\varphi \equiv u, v, \theta, q)$ at the lower (surface) and upper (entrainment) boundaries, respectively; $h$ is the boundary-layer height, while $A d v_{\varphi}$ is the advection of the corresponding quantity of interest. The chevrons " $\langle\varphi\rangle$ " represents the variables within the mixed layer. For a more complete description of the mixed-layer governing equations, see van Heerwaarden et al. (2009) and Ouwersloot et al. (2012). In what follows, we incorporate the most physically sound roughness-sublayer model (Harman and Finnigan 2007; 2008) in the surface scheme of our modelling system (following the concept of Harman (2012)). We calculated the surface fluxes in Eq. 3.1 as follows:

$$
\left(\overline{w^{\prime} \varphi^{\prime}}\right)_{s}=\frac{\left(\varphi_{s}-\varphi\left(z_{r}\right)\right)}{r_{a \varphi}+r_{s \varphi}}
$$

where $\varphi_{s}$ and $\varphi\left(z_{r}\right)$ are the mean vector (wind velocity) and scalar (potential temperature, specific humidity) quantities at roughness length $\left(z_{0 \varphi}\right)$ and at a given reference height within the RSL $\left(z_{r}\right)$. For momentum $z_{0 \varphi} \equiv z_{0 M}$, while for scalars $z_{0 \varphi} \equiv z_{0 H}$. The aerodynamic resistance in Eq. 3.2 is calculated at $z_{r}$ and is related to the drag coefficient $\left(C_{\varphi}\right)$ and the mean wind speed $(|U|)$ at the same height:

$$
r_{a \varphi}=\left(C_{\varphi}\left(z_{r}\right)\left|U\left(z_{r}\right)\right|\right)^{-1} .
$$

The stomatal resistance, $r_{s \varphi}$, in Eq. (3.2) is equal to zero for momentum and heat. Its definition and computation for moisture is presented and explained in van Heerwaarden et al. (2009).

The influenced $C_{\varphi}\left(z_{r}\right)$ and $\varphi\left(z_{r}\right)$ due to the canopy presence are calculated using the following expressions:

$$
\begin{array}{r}
C_{\varphi}\left(z_{r}\right)=\kappa^{2} \frac{1}{\left[\ln \left(\frac{z_{r}}{z_{0 M}}\right)-\Psi_{M}\left(\frac{z_{r}}{L}\right)+\Psi_{M}\left(\frac{z_{0 M}}{L}\right)+\hat{\Psi}_{M}\left(z, d_{t}, L\right)\right]} \\
\frac{1}{\left[\ln \left(\frac{z_{r}}{z_{0 \varphi}}\right)-\Psi_{\varphi}\left(\frac{z_{r}}{L}\right)+\Psi_{\varphi}\left(\frac{z_{0 \varphi}}{L}\right)+\hat{\Psi}_{\varphi}\left(z, d_{t}, L\right)\right]},
\end{array}
$$

and

$$
\varphi\left(z_{r}\right)=\varphi_{s}-\frac{\left(\overline{w^{\prime} \varphi^{\prime}}\right)_{s}}{\kappa u_{*}}\left[\ln \left(\frac{z_{r}}{z_{0 \varphi}}\right)-\Psi_{\varphi}\left(\frac{z_{r}}{L}\right)+\Psi_{\varphi}\left(\frac{z_{0 \varphi}}{L}\right)+\hat{\Psi}_{\varphi}\left(z, d_{t}, L\right)\right],
$$

where $\kappa$ is the von-Kármán constant of 0.41 (Högström 1989). The friction velocity is computed as:

$$
u_{*}=\sqrt{C_{M}\left(z_{r}\right)}\left|U\left(z_{r}\right)\right|
$$


The functions: $\Psi_{M}\left(\frac{z_{r}}{L}\right), \Psi_{M}\left(\frac{z_{0}}{L}\right), \Psi_{\varphi}\left(\frac{z_{r}}{L}\right), \Psi_{\varphi}\left(\frac{z_{0 \varphi}}{L}\right)$ are the integrated diabatic stability functions for momentum and scalars, while $\hat{\Psi}_{M}\left(z_{r}, d_{t}, L\right)$ and $\hat{\Psi}_{\varphi}\left(z_{r}, d_{t}, L\right)$ represent the roughness sublayer functions for momentum and scalars (Harman and Finnigan 2007; 2008). Stability-dependent roughness lengths for momentum and other scalars $\left(z_{0 M}\right.$ and $z_{0 \varphi}$, respectively) included in Eqs. 3.4 and 3.5 are described in detail in Harman (2012).

The displacement height, $d_{t}$, in Eqs. 3.4 and 3.5 is defined as the distance from the conventional displacement plane, at actual height, $d$, to the canopy top, at actual height $h_{c}: d_{t}=h_{c}-d$ (see Fig. 3.1). Based on Harman and Finnigan (2017), $d_{t}$ is calculated as follows:

$$
d_{t}=\beta^{2} L_{c}
$$

where, $L_{c}$, is canopy adjustment length scale, defined as:

$$
L_{c}=\left(c_{d} a\right)^{-1}
$$

where $a$ is the canopy's leaf area density which is assumed to be constant with height (Harman and Finnigan 2007), while $c_{d}$ is the leaf drag coefficient, calculated from the observations at the canopy top $\left(c_{d}=u_{*}^{2} /(|U|)^{2}\right)$. The canopy adjustment length scale (Eq. 3.8) is defined as a measure of the distance over which an internal boundary layer with no prior knowledge of a tall canopy would need to equilibrate (adjust) to the presence of a canopy (Belcher et al. 2003; Harman and Finnigan 2007) For the given CHATS experiment, Shapkalijevski et al. (2016) have shown that $L_{c}=16 \mathrm{~m}$ under near-neutral and weakly-unstable conditions. Under strongly-unstable conditions $L_{c} \approx 10 \mathrm{~m}$, while under strongly-stable conditions $L_{c}>20 \mathrm{~m}$. Another critical stability-dependent variable in Eq. 3.7 is $\beta$, which indicates the ratio between the friction velocity and the mean wind speed at canopy top $\left(\beta=\frac{u_{*}}{|U|}\right)$. Based on our CHATS analysis (Shapkalijevski et al. 2016), we find that under weakly-unstable, near-neutral and weakly-stable atmospheric conditions $\beta$ has constant value of 0.3, consistent with Harman and Finnigan (2007; 2008). Under stronglyunstable conditions, this variable increases up to 0.4 , while under strongly-stable conditions it decreases to nearly 0.25 . Based on estimates at the CHATS site, we assume the values of 0.3 and $16 \mathrm{~m}$ for $\beta$ and $L_{c}$, respectively. The sensitivity of the calculated surface fluxes and boundary state variables to the values of $\beta$ and $L_{c}$ is presented and discussed in sections 3.4 and 3.5.

Finally, the RSL functions $\hat{\Psi}_{M}\left(z_{r}, d_{t}, L\right)$ and $\hat{\Psi}_{\varphi}\left(z_{r}, d_{t}, L\right)$, are non-linear integrals, which are solved numerically. For a detailed theoretical description and derivation of these RSL functions, see Harman and Finnigan (2007; 2008).

\subsubsection{Research strategy}

To initialise and validate our modelling system, we selected observations of a representative day from the second phase of the CHATS campaign (from 13 May to 12 June) focusing on the walnut 
trees after leaf-out (fully vegetated canopy). The representative case is based on two requirements that the data satisfied: $i$ ) well-mixed conditions and $i$ ) well-developed RSL. Our assumption of a well-mixed boundary layer is justified for sunny (cloudless) days characterised by convective conditions. Moreover, the LIDAR data (see figures in supplementary material) showed a quite homogeneous signal, which in the absence of radiosoundings implies well-mixed conditions up to $500 \mathrm{~m}$ height at noon (12:00 LT). In order to ensure the maximum influence (fetch) of the canopy on the atmospheric flow, leading to a potentially well-developed RSL, we selected data with southerly predominant winds, since the measurement tower was placed at the northernmost part of the orchard field (Patton et al. 2011). Based on these requirements, we selected observations from 27 May 2007 at CHATS. To test the robustness of the model results, we also analysed an additional day (31 May 2007) with different wind forcing (northerly varying to southerly winds in the course of the day).

Several systematic experiments were performed, in which the representation of the drag coefficient and the impact of the RSL on mean gradients (Eqs. 3.4-3.5), as well as the inclusion of various large-scale forcing were varied. The standard MOST runs (abbreviated as 'M') were performed by omitting the roughness sublayer functions in Eqs. 3.4-3.5. The large-scale forcing consists of mean vertical velocity subsidence, advection of cold and moist air, and increased boundary layer drying due to a drier free troposphere (see next paragraph). Table 3.1 summarises the processes included in the numerical experiments.

Table 3.1: Numerical model runs; description and abbreviations.

\begin{tabular}{l|c|c|c|c}
\hline Experiment abbreviation & RSL (R) or MOST (M) & Subsidence (S) & Advection (A) & FT drying (D) \\
\hline MXL+R & R & - & - & - \\
MXL+RS & R & S & - & - \\
MXL+RSA & R & S & A & - \\
MXL+RSAD & R & S & A & D \\
MXL+MSAD & M & S & A \\
\hline
\end{tabular}

The numerical experiment which does not take subsidence into account has prescribed zero subsidence (no divergence of the mean horizontal wind), while the numerical experiments with subsidence have imposed constant divergence of the mean horizontal wind (Appendix 3A). Based on the observed temporal evolution of the potential temperature and specific humidity at $29 \mathrm{~m}$, we set constant advective cooling and moistening at specific moment in time in our numerical experiments (Appendix 3A). No advection of momentum has been imposed in the momentum budget. Furthermore, to represent the increased BL drying from the free troposphere we modified the specific humidity lapse rate in the free troposphere $\left(\gamma_{q}\right)$ depending on the BL-height (Appendix $3 \mathrm{~A}$ ). For instance, to represent the observed temporal evolution of the specific humidity at $29 \mathrm{~m}$ during the day on 27 May 2007, we prescribed a modification of the $\gamma_{q}=10^{-4} \mathrm{~kg} \mathrm{~kg}^{-1} \mathrm{~m}^{-1}$ when the BL-height reaches $450 \mathrm{~m}$ (based on observations), while the initial $\gamma_{q}$ was set equal to 0 units 
(see Table 3A.1).

The numerical experiments started at 08:00 local time (LT), which is equivalent to 15:00 coordinated universal time (UTC), and lasted for nine hours. In the absence of initial measurements at the residual layer (roughly $350 \mathrm{~m}$ ); we imposed the upper boundary conditions of the model to optimise the representation of the temporal evolution of the potential temperature, specific humidity, wind direction and boundary-layer height (Table 3A.1 and 3A.2 in Appendix 3A). We used the observations at the highest measurement level at the tower (29 $\mathrm{m}$ above ground surface) to evaluate the model results away from the canopy, where the RSL effects are minimal.

Furthermore, we put special emphasis on validating the modelled quantities at the canopy top $\left(z=z_{r}=d_{t}\right.$ ) and compared them with the corresponding observations at the same height. We selected the canopy top (10 $\mathrm{m}$ above the ground surface) as a reference level due to the largest expected RSL effects on the flow (Harman and Finnigan 2007; 2008). We note that the area of the orchard is rather small $(\sim 1 \mathrm{~km} 2)$ to be capable of influencing the development of the boundarylayer dynamics (Schmid 2002). However, in the model, we extrapolated the characteristic surface fluxes and mean gradients, assuming that the area of this orchard is sufficient to drive the main processes at the CBL dynamics.

Finally, the initial value of $z_{0 M}=0.7 \mathrm{~m}$ used in all the numerical runs (Appendix $3 \mathrm{~A}$ ) was estimated based on the approach developed by Raupach (1994) for a LAI of 2.5 and $\beta=0.3$. Thus, the initial value of the roughness length for scalars, $z_{0 M}=0.095 \mathrm{~m}$ (see Table 3A.1), is calculated as $\ln \left(\frac{z_{0 M}}{z_{0 H}}\right)=2$ (see Physick and Garratt 1995). For the standard MOST runs (MXL+MSAD), we used invariant (fixed) $z_{0 M}$ and $z_{0 \varphi}$ with values equal to their corresponding initial values, while when including the RSL, we used stability dependent formulation for $z_{0 M}$ and $z_{0 \varphi}$ (Harman and Finnigan 2007; 2008).

\subsection{Model validation}

\subsubsection{Radiation and surface energy balance}

We start our analysis by evaluating the modelling system to represent the observations of the selected study cases. Figure 3.2a,b shows the observed and modelled components of the net radiation: downwelling $(\downarrow)$ and upwelling $(\uparrow)$ shortwave (SW) and longwave (LW) radiation fluxes above the canopy (measured at $6 \mathrm{~m}$ above the canopy top). The various radiation components are well reproduced by the model.

Figure 3.2c,d shows the four terms of the surface energy balance $(\mathrm{Rn}=\mathrm{SH}+\mathrm{LE}+\mathrm{G})$ for both cases, respectively. The surface fluxes in the model are calculated from the differences between the surface and the roughness sublayer (reference height) values of the mean quantities and the transfer coefficients for momentum and scalars (see section 3.2.2, Eq. 3.2). While the net radiation fluxes compare satisfactorily with the observations, the modelled daily averaged values of SH and LE are overestimated: around $60 \%$ and $20 \%$ larger than the observed LE and SH, 


\begin{tabular}{|c|c|c|c|}
\hline 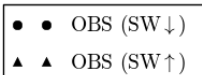 & $\begin{array}{l}\text { - } \text { OBS }(\mathrm{LW} \downarrow) \\
\text { - } \mathrm{OBS}(\mathrm{LW} \uparrow)\end{array}$ & $\begin{array}{ll}-\mathrm{MXL}+\mathrm{RSAD}(\mathrm{SW} \downarrow) \\
- \text { MXL+RSAD }(\mathrm{SW} \uparrow)\end{array}$ & $\begin{array}{ll}\cdots \cdot & \mathrm{MXL}+\operatorname{RSAD}(\mathrm{LW} \downarrow) \\
\cdots & \mathrm{MXL}+\operatorname{RSAD}(\mathrm{LW} \uparrow)\end{array}$ \\
\hline
\end{tabular}
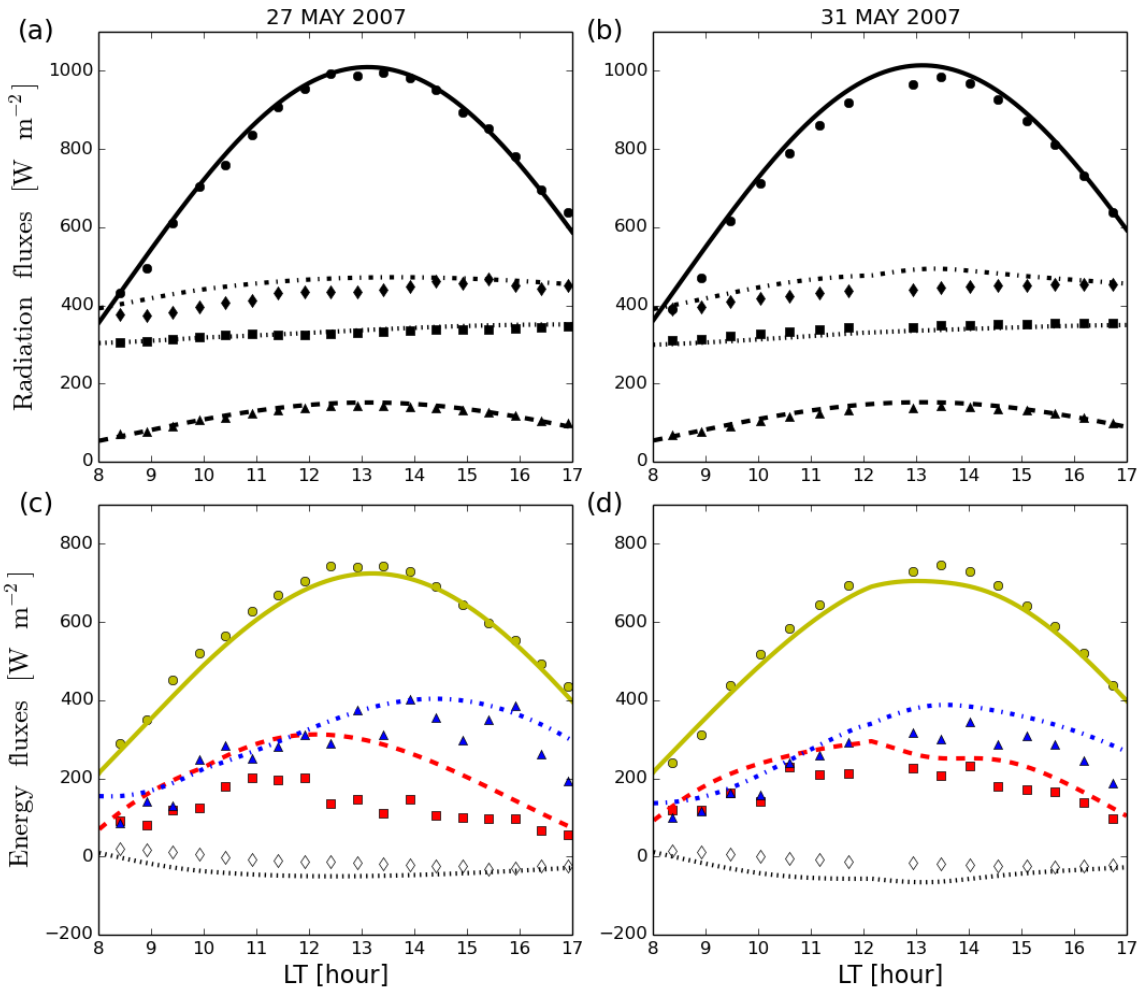

\begin{tabular}{|c|c|c|c|c|c|c|}
\hline - $\mathrm{OBS}(\mathrm{Rn})$ & $\Delta$ & $\triangle$ OBS $(\mathrm{SH})$ & - & $\mathrm{MXL}+\mathrm{RSAD}(\mathrm{Rn})$ & $\cdots$ & $\mathrm{MXL}+\mathrm{RSAD}(\mathrm{LE})$ \\
\hline - OBS $(\mathrm{G})$ & $\diamond$ & $\diamond \quad$ OBS $(\mathrm{LE})$ & $=-$ & $\mathrm{MXL}+\mathrm{RSAD}(\mathrm{SH})$ & $\cdots \cdots \cdot$ & $\mathrm{MXL}+\operatorname{RSAD}(\mathrm{G})$ \\
\hline
\end{tabular}

Figure 3.2: Observed and modelled radiation and surface energy balance components: (a) and (b) diurnal evolution of downwelling shortwave radiative flux ( $S W \downarrow)$, upwelling shortwave radiat-

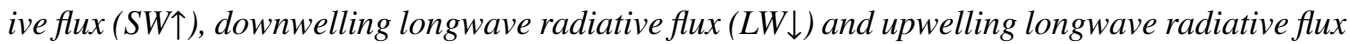
$(L W \uparrow) ;(c)$ and $(\boldsymbol{d})$ diurnal evolution of sensible heat flux (SH), latent heat flux (LE), the ground flux $(G)$ and net radiation $(R n)$ (all in $W^{-2}$ ). Observed quantities are measured at $6 m$ above the canopy top. LT is local time (UTC-7). Sunrise was at 06:30 LT and sunset was at 19:30 LT. 


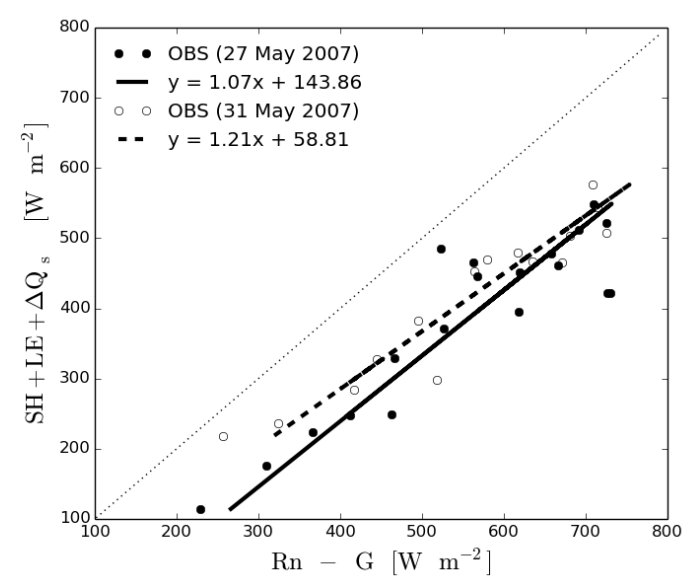

Figure 3.3: Observed non-closure of the surface energy balance on 27 and 31 May 2007 during the CHATS experiment.

respectively for both case studies (27 and 31 May 2007). The average daily difference in the modelled and observed ground flux is up to $5 \mathrm{~W} \mathrm{~m}^{-2}$. The diurnal variations in the observed LE and SH are well captured by the model, for instance the rapid decay of SH towards the end of the day relative to LE.

Our explanation of this overestimation is the frequently observed imbalance of the observed surface energy system (Foken 2008b). This hypothesis is corroborated by an observed daily average difference of up to $-30 \%$ of $\mathrm{SH}+\mathrm{LE}+\Delta Q_{s}$ compared to $\mathrm{Rn}-\mathrm{G}$ for the case of 27 May and $-20 \%$ on 31 May (Fig. 3.3), even when the heat storage contribution $\left(\Delta Q_{s}\right)$ is included in the observed SEB (up to $5 \%$ energy input in the total balance). The $\Delta Q_{s}$ is the sum of the sensible $\left(\Delta Q_{a}\right)$ and latent $\left(\Delta Q_{w}\right)$ heat storage in the air column (including the canopy space) below the flux measurements by eddy-covariance (EC). The method used to calculate $\Delta Q_{s}$ from the observed potential temperature and specific humidity at the levels within and above the canopy, but below the height of EC observations, is based on that described by McCaughey and Saxton (1988) and later used in Oliphant et al. (2004). Note that presented G accounts for the heat storage in the soil, as calculated following Oliphant et al. (2004). The heat stored in the biomass and the energy used in the photosynthesis are neglected in our case, since according to Thom et al. (1975), Ohta et al. (Ohta1999) and Jacobs et al. (2007) these two terms are negligibly small (less than $2 \%$ of total $\mathrm{Rn}$ ). The values of the surface energy imbalance at CHATS are similar to those found by a number of other observational studies, showing an average of up to $20 \%$ surface energy imbalance, as listed in section 3.7 of Foken (2008b). With regard to our own research, it is important to note that related to this non-closure of the observed SEB, the observed SH and LE are too low, so the modelled SH and LE are more likely to be the correct values. 
The comparison presented here confirms that our modelling system is capable of reproducing the diurnal variations in radiation with sufficient accuracy. As in many other studies (see Foken 2008b), the observed surface energy balance remains not closed, but with the deviations of similar magnitude as observed in other studies above high canopy.

\subsubsection{CBL dynamics}

Figure 3.4 shows the observed and modelled diurnal evolution of the boundary-layer height, mixed-layer potential temperature and specific humidity for the case of 27 May 2007. The boundary-layer height (Fig. 3.4a), $h$, increases during the morning hours from $350 \mathrm{~m}$ to up to $500 \mathrm{~m}$ at around 11:00 LT, after which $h$ remains almost constant before it starts to decay at around 14:00 LT. In the absence of data on the vertical profiles of potential temperature and specific humidity in the mixed layer and the entrainment zone, we are unable to judge whether this more rapid growth until 11:00 LT is due to a progressive growth of the CBL into a residual layer above the canopy (Ouwersloot et al. 2012). Since our aim is to study the RSL effects on CBL dynamics, here we focus our analysis to the numerical experiments described above.

It is important to mention that $h$, as observed by the LIDAR backscatter data, is very sensitive to the morning-noon transition (08:00 - 10:00 LT) and late afternoon-evening (after 16:00 LT) transition conditions. This is due to possible non-uniform backscatter profiles, which can contain multiple maximum gradients, impairing the ability of the automated method to retrieve $h$ (see Appendix 3C). Therefore, the accuracy of the observations of $h$ is better under well-mixed conditions (from 10:00 to 16:00 LT in our case). During this period, only the model runs that take into account the subsidence and advective cooling (MXL+RSAD and MXL+RSA) capture the evolution (relatively steady) of the $h$ sufficiently well after the morning transition (Fig. 3.4a, in connection with Table 1). This result implies a significant influence of the subsidence, and to a lesser extent the effects of advective cooling, on boundary-layer growth for the given case. Figure 3.4a also shows that the effect of the RSL on the evolution of $h$ is insignificant (MXL+RSAD vs MXL+MSAD).

The role of the large-scale advective cooling on the CBL dynamics was also recorded through the diurnal evolution of the potential temperature (Fig. 3.4b) at $29 \mathrm{~m}$ above the ground. The level of $29 \mathrm{~m}$ is considered to be representative of the mixed-layer values, since it is either located within the mixed layer or in the upper part of the surface layer, where deviations compared to mixed-layer values are small. Therefore, we employ it as the most representative of the mixedlayer characteristics. Between 10:00 LT and 12:00 LT, a non-local advective cooling process resulted in a slowdown in the increase of the potential temperature. We hypothesize that the rapid temperature drop before noon is related to the advection of cold air, due to a sea-breeze front, which is frequently observed around noon at the CHATS site (Mayor et al. 2011). We took this process into account in our numerical experiment (MXL+RSA) by imposing a constant advection of cold air between 10:00 LT and 17:00 LT (Table 3.1). The strength of the advective 

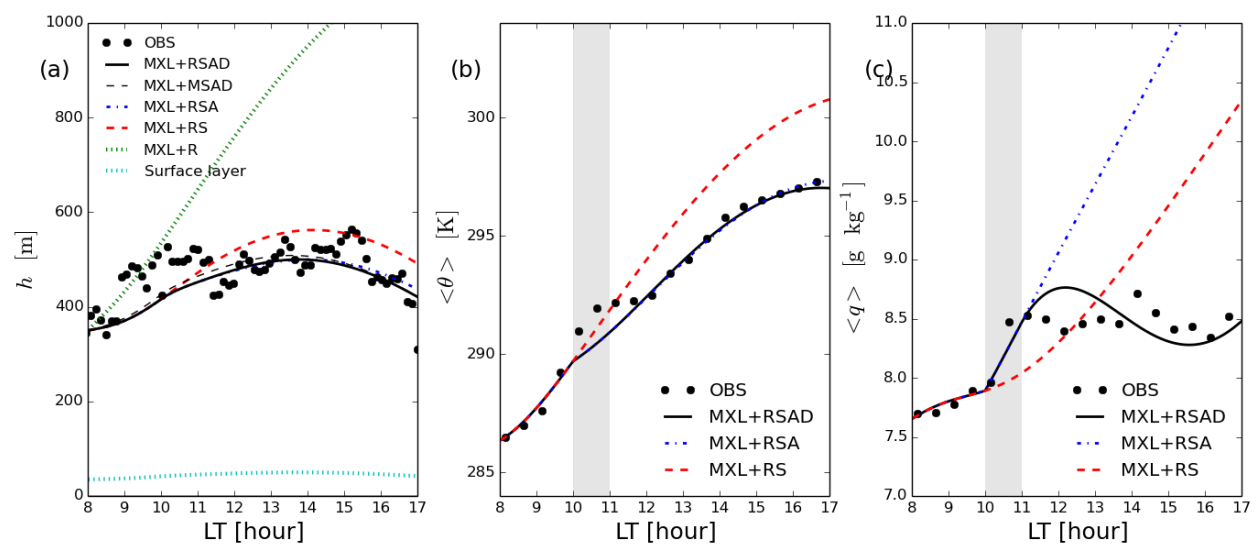

Figure 3.4: Temporal evolution of the observed versus modelled mixed-layer quantities on 27 May 2007: (a) boundary layer height $(h)$ (b) potential temperature, $\langle\theta\rangle$, and (c) specific humidity, $\langle q\rangle$. Observations are denoted by black symbols. $\langle\theta\rangle$, and $\langle q\rangle$ are measured at $29 \mathrm{~m}$ above the ground surface and $h$ is obtained from LIDAR data (Mayor et al. 2011; Patton et al. 2011). The numerical experiments are described in Table 3.1. Shaded areas in (b) and (c) indicate the cooling and moistening periods of the atmospheric boundary layer.

cooling in the model was arbitrarily chosen to provide the best representation of the observed mixed-layer quantities (Table 3A.1, Appendix 3A). As Fig. 3.4b shows, while taking only surface forcings, entrainment processes and subsidence into account does not suffice to represent this case (experiment MXL+RS), the potential temperature evolution is captured well if the advection is taken into account (experiment MXL+RSA) as well.

Similar behaviour of the diurnal evolution of the specific humidity at $29 \mathrm{~m}$ above the ground surface was observed (Fig. 3.4c). Here, the large-scale advective process is displayed by a significant jump in the magnitude of the specific humidity (from $7.9 \mathrm{~g} \mathrm{~kg}^{-1}$ to as much as $8.5 \mathrm{~g} \mathrm{~kg}^{-1}$ ) immediately after 10:00 LT. In the absence of observed specific-humidity profiles, we hypothesise that this increase in moisture content is due to an air mass transported by the sea-breeze front coming from the bay area (east and southeast). It is also possible that during the morning transition this sudden change is caused by the existence of a residual layer, which becomes connected to a growing shallow layer (Ouwersloot et al. 2012). However as mentioned before, since there are no data to explain the latter, but also because main focus of this study is the effects of the RSL on the CBL dynamics, we limited our analysis to the numerical experiments described above. After this increase, $q$ remains steady until the end of the day (17:00 LT). We related this behavior of $q$ after noon to the drying associated with the entrainment of free tropospheric (drier) air into the boundary layer, which can be driven by returned flow over the complex topography (Bianco et al. 2011). Based on the observed $q$ in the hours after 11:00 LT, the transport of dry air from 

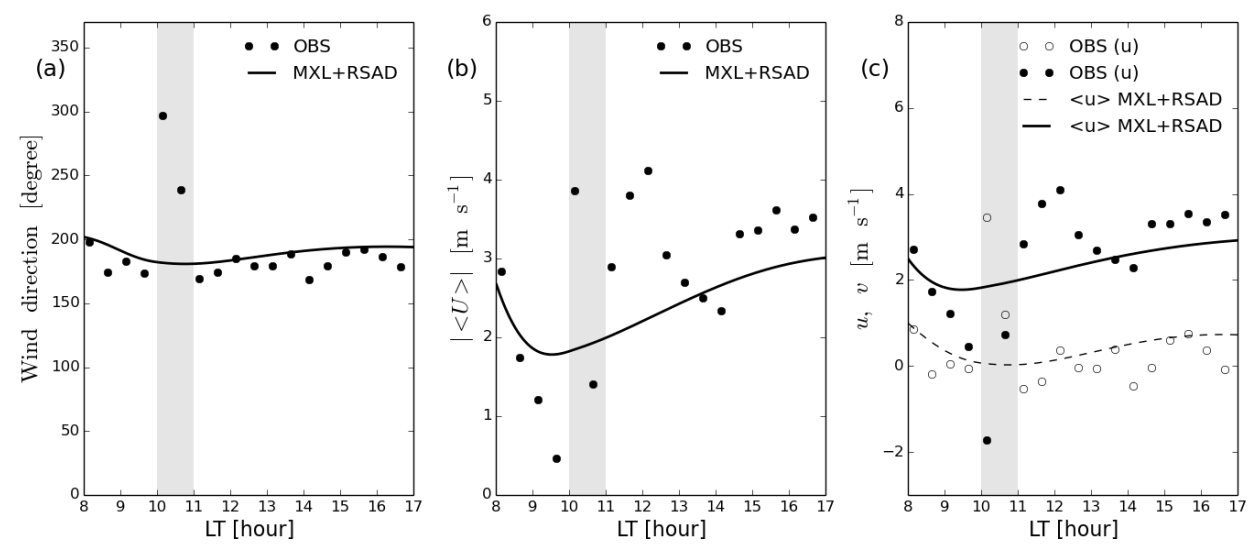

Figure 3.5: Temporal evolution of the observed versus modelled boundary-layer dynamics at 29 $m$ above the ground surface: (a) mixed-layer wind direction, $(\boldsymbol{b})$ calculated modulus of the mixedlayer wind speed, (c) mixed-layer wind speed components. Shaded area indicates the period when the wind change occurs.

the free troposphere is dominant, preventing the rise in the specific humidity, which results in a relatively constant value. The diurnal evolution of the specific humidity is well represented by the model run that takes the subsidence, advection and drying from the free troposphere into accounts (MXL+RSAD). On the other hand, the model runs which do not take the drying (MXL+RSA) and the advection and drying $(\mathrm{MXL}+\mathrm{RS})$ into account overestimate the specific humidity after 11:00 LT.

The analysis presented in Fig. 3.4 shows that the complex boundary-layer structure at the CHATS site is highly dependent on the large-scale effects, including subsidence, advective cooling and moistening, as well as entrainment of dry air from the free troposphere.

The observed diurnal variability of the wind enables us to further verify the role of the largescale forcing and the local canopy. Here, we compare the observed and modelled temporal evolution of the wind direction, individual wind speed components and absolute wind velocity (Fig. 3.5). The model is well able to represent the observed temporal evolution of wind, except for the period between 10:00 and 11:00 PLT, when outliers are present in the observed wind components (Fig. 3.5c) and, consequently, the wind direction (Fig. 3.5a). These outliers are associated with the sharp changes in the wind forcing (northerly winds present between 10:00 and 11:00 LT), a phenomenon observed daily before noon throughout whole campaign (based on observed time series) (see also Zaremba and Carroll 1999). Combining the individual wind components closely approximates the wind speed, which displays an almost constant acceleration during the day (Fig. 3.5b) and (after 11:00 LT) an almost constant friction velocity (see Figure 3.6c).

The results of the case study of 27 May 2007 are corroborated by those of the case study of 
Table 3.2: Calculated mean absolute error (MAE) of MXL+MSAD and MXL+RSAD numerical runs with respect to observations. The values of the MAE are presented in units of the corresponding quantities; the values in brackets show the model percentage of the MAE values relative to the daily means (between 08:00 and 17:00 LT) of the observed quantities respectively.

\begin{tabular}{|c|c|c|c|c|c|c|c|c|}
\hline & $\begin{array}{l}\left|U\left(z_{r}\right)\right| \\
{\left[\mathrm{m} \mathrm{s}^{-1}\right]}\end{array}$ & $\begin{array}{c}C_{M}\left(z_{r}\right) \\
{[-]}\end{array}$ & $\begin{array}{c}u_{*} \\
{\left[\mathrm{~m} \mathrm{~s}^{-1}\right]}\end{array}$ & $\begin{array}{c}\theta\left(z_{r}\right) \\
{[\mathrm{K}]}\end{array}$ & $\begin{array}{c}q\left(z_{r}\right) \\
{\left[\mathrm{g} \mathrm{kg}^{-1}\right]}\end{array}$ & $\begin{array}{c}\mathrm{SH} \\
{\left[\mathrm{W} \mathrm{m}^{-2}\right]}\end{array}$ & $\begin{array}{c}\mathrm{LE} \\
{\left[\mathrm{W} \mathrm{m}^{-2}\right]}\end{array}$ & $\begin{array}{c}h \\
{[\mathrm{~m}]}\end{array}$ \\
\hline $\begin{array}{l}\text { Mean observed } \\
\text { MXL+MSAD }\end{array}$ & 1.45 & 0.11 & 0.44 & 293.86 & 8.60 & 128.46 & 250.88 & 473.06 \\
\hline Mean model & 1.00 & 0.20 & 0.32 & 294.37 & 8.49 & 222.53 & 313.72 & 463.84 \\
\hline $\begin{array}{l}\text { MAE } \\
(\%)\end{array}$ & $\begin{array}{c}0.50 \\
(34.90)\end{array}$ & $\begin{array}{c}0.10 \\
(88.75)\end{array}$ & $\begin{array}{c}0.13 \\
(31.30)\end{array}$ & $\begin{array}{c}0.47 \\
(0.16)\end{array}$ & $\begin{array}{c}0.22 \\
(2.62)\end{array}$ & $\begin{array}{c}87.18 \\
(67.82)\end{array}$ & $\begin{array}{c}59.22 \\
(23.60)\end{array}$ & $\begin{array}{l}34.09 \\
(7.18)\end{array}$ \\
\hline MXL+RSAD & & & & & & & & \\
\hline Mean model & 1.64 & 0.06 & 0.03 & 294.01 & 8.81 & 217.38 & 307.26 & 457.80 \\
\hline $\begin{array}{l}\text { MAE } \\
(\%)\end{array}$ & $\begin{array}{c}0.34 \\
(24.06)\end{array}$ & $\begin{array}{c}0.06 \\
(41.53)\end{array}$ & $\begin{array}{c}0.15 \\
(34.20)\end{array}$ & $\begin{array}{c}0.41 \\
(0.24)\end{array}$ & $\begin{array}{c}0.37 \\
(4.41)\end{array}$ & $\begin{array}{c}81.81 \\
(63.68)\end{array}$ & $\begin{array}{l}52.86 \\
(21.07)\end{array}$ & $\begin{array}{l}35.97 \\
(7.60)\end{array}$ \\
\hline
\end{tabular}

31 May 2007 (not shown), showing similar patterns and structure of the CBL dynamics in both cases.

In summary, our modelling system is capable of reproducing the land-canopy-atmosphere characteristics of the case studies with satisfactory accuracy at a height well above the canopy. In the following section, we study the impact of the canopy on the boundary-layer state variables within the roughness sublayer near the canopy top.

\subsection{The wind in the RSL and effects on bulk momentum budget}

Figure 3.6 shows the observed and modelled temporal evolution of the mean wind speed, drag coefficient and friction velocity at the canopy top. The numerical experiment MXL+RSAD of the coupled modelling system satisfactorily represents the evolution of the wind at this level, while omitting the RSL effects (MXL+MSAD) results in underestimation of the wind speed (reaching a daily average of up to $50 \%$; Fig. 3.6a). This is in agreement with previous studies based on comparisons of observed and modelled wind profile (Physick and Garratt, 1995; Harman and Finnigan, 2007). The main effect of the canopy is a modification of the drag. Omitting the RSL effects (MXL+MSAD vs MXL+RSAD) results in significant overestimation of $C_{M}$ by a factor of up to four (Fig. 6b), in accordance with the analysis provided by De Ridder (2010).

Both the MXL+RSAD and MXL+MSAD model runs, i.e., with and without the effects of the RSL included, underestimate $u_{*}$ by about $20 \%$ (Fig. 3.6c). Like Physick and Garratt (1995), we found small RSL effects on the modelled friction velocity in the case studies $(6 \%)$. The similarity between the friction velocities is due to compensating effects of the drag coefficient and the wind speed modulus (Eqs. 3.2-3.6). Both $C_{M}$ and $|U|$ are altered in opposite directions when the RSL 
(a)
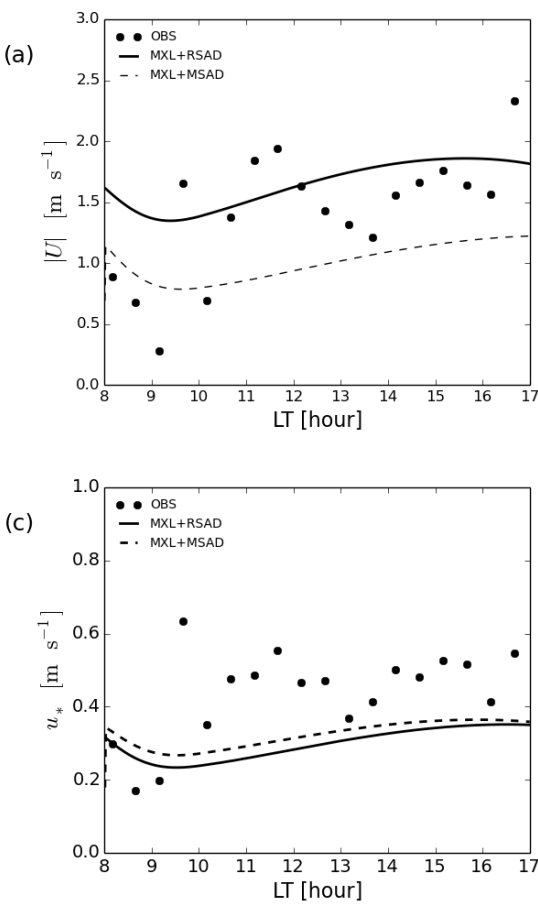

(b)
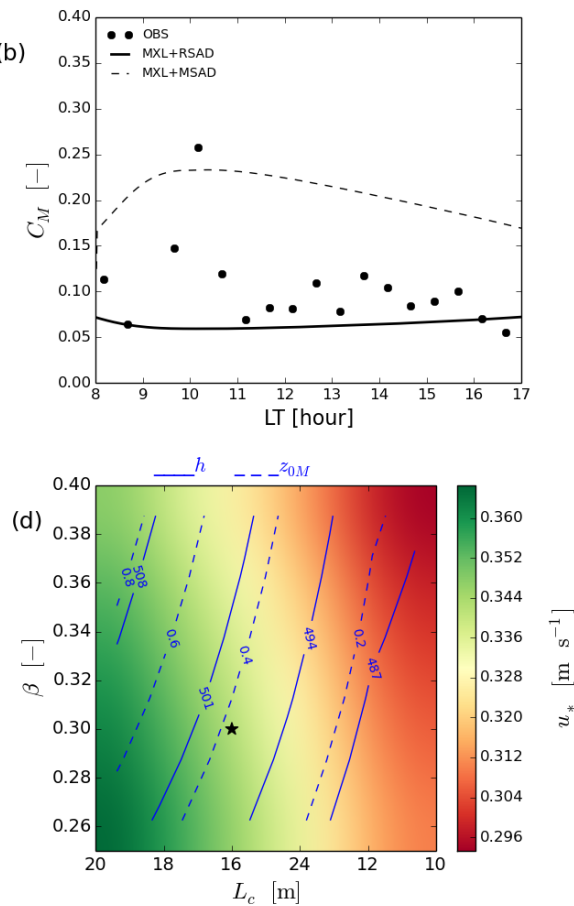

Figure 3.6: Observed versus modelled modulus of the wind speed (a), momentum drag coefficient (b), friction velocity (c) with and without the RSL effects (solid line and dashed lines, respectively) at $10 \mathrm{~m}$ above the ground surface (equal to average tree heights, $h_{c}=10 \mathrm{~m}$ ). (d) Sensitivity of the friction velocity (colour scale), roughness length for momentum $\left(z_{0 M}[\mathrm{~m}]\right.$, dashed line) and boundary-layer height ( $h[\mathrm{~m}]$, full line) at 13:00 LT to changes in the values of $\beta$ and $L_{c}$. The black asterisk indicates the conditions for the case study of 27 May 2007.

representation is introduced (Eq. 3.4 and 3.5), with magnitudes that fit the observation (Fig. 3.6a,b), thus leading to a relatively unchanged $u_{*}$ (see Eq. 3.6, and Table 3.2).

Table 3.2 shows the overview of the performance of the two numerical experiments with and without RSL representation (MXL+RSAD and MXL+MSAD, respectively) with respect to observations, as quantified by the mean absolute error (MAE). The numerical experiment with RSL representation performs better than the numerical experiment that omits the RSL when representing the wind speed and the drag at canopy height. Both numerical experiments (MXL+RSAD and MXL+MSAD) however underestimate the observed friction velocity. The small difference in magnitude of the friction velocity between the experiments is due to use of different roughness length and displacement height formulation: as stability dependent variables in MXL+RSAD, and as fixed parameters estimated under neutral condition in MXL+MSAD. MXL+RSAD also represents the potential temperature better than MXL+MSAD at the same level, but slightly over- 


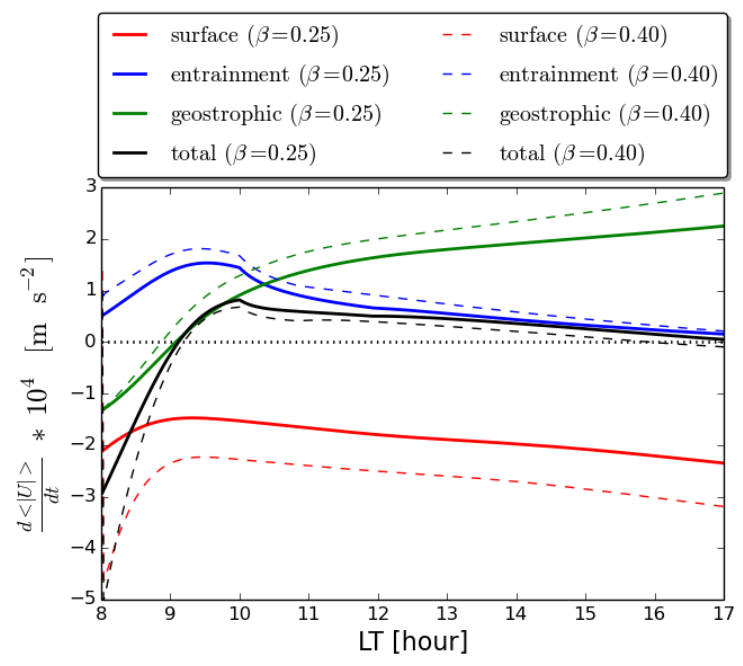

Figure 3.7: Budget of the mixed-layer wind speed components $\langle|U|>$ based on different canopyflow forcing.

estimate the specific humidity (see Sect. 3.5). As expected, the largest MAEs are found for the surface fluxes (e.g. 60\% MAE for SH with respect to the mean observed SH). Again, note that the observed SH and LE are not the 'true' surface fluxes since the energy balance is not closed (Fig. 3.3).

In order to extend and generalise our results, we performed a parameter-space sensitivity analysis on two stability-dependent scales in the RSL formulation: $L_{c}$ and $\beta$ (see also section 3.2.2). Figure $3.6 \mathrm{~d}$ summarises the results of the sensitivity analysis at 13:00 LT. The variations in $\beta(0.25 \leqslant \beta \leqslant 0.4)$ and $L_{c}\left(10 \leqslant L_{c} \leqslant 20 \mathrm{~m}\right)$ have a significant impact on $z_{0 M}$ and $u_{*}$, but a relatively small impact on $h$. We find that $u_{*}$ is sensitive to the changes in $\beta$ and $L_{c}$ with a maximum variation at 13:00 LT of up to $25 \%\left(0.29 \leqslant u_{*} \leqslant 0.37 \mathrm{~m} \mathrm{~s}^{-1}\right)$ with respect to the case study value $\left(u_{*}=0.32 \mathrm{~m} \mathrm{~s}^{-1}\right)$ for the range of conditions investigated here. In our analysis, varying these scales, dependent on stability (based on the CHATS data), results in $h$ variation of up to 6 $\%$ (Fig. 3.6d).

We further extend our analysis of the impact of the canopy-related parameters on the atmospheric flow by studying their relative contribution to the momentum budget, compared to other contributions, e.g. entrainment or geostrophic forcing (Appendix 3B). For this, we keep $L_{c}$ equal to $16 \mathrm{~m}$ and in the first experiment, we set $\beta=0.25$ (typical for more stratified conditions), while in the second experiment we set $\beta=0.40$ (typical for unstable conditions). Varying $L_{c}$ did not yield relevant differences in the wind budget (not shown). Figure 3.7 shows that on average the momentum tendency due to surface stress is approximately $25 \%$ larger for $\beta=0.40$ than when 
$\beta=0.25$. This enhanced tendency is partially compensated for by an increase in geostrophic forcing through the whole day and, to a lesser degree, entrainment. This results in a similar total momentum tendency in both cases.

Figure 3.7 also shows the tendencies of the three components of the total wind-speed budget (Appendix 3B): surface forcing, the momentum entrainment, and the geostrophic forcing. The surface forcing, combining the surface stress and canopy drag, always leads to a negative tendency in the momentum, while entrainment from free-tropospheric air results in a positive tendency. In the case under study, the tendencies of the ageostrophic components are also usually positive. The resulting total momentum tendency is positive after 09:00 LT.

In summary, although the variation of the RSL scale $\beta$ strongly affects the surface shear partitioning in the momentum budget, the total momentum tendency remains relatively unchanged due to compensation by the geostrophic and entrainment contribution. This means that the imposed pressure gradient force, integrated over the boundary-layer depth is balanced by the surface friction and momentum entrainment. Since the boundary-layer depth is similar between the both runs, then pressure gradient force and momentum entrainment are altered to balance the differences in the surface shear between the runs.

\subsection{Heat and moisture}

The impact of the RSL on the potential temperature and specific humidity at canopy-top level and their respective surface heat fluxes is presented in Fig. 3.8 and Fig. 3.9. Here, similar analyses were performed as for momentum in the previous section. The modelled potential temperature at this level is in good agreement with the observations. The suppressed increase in potential temperature before noon is caused by the large-scale advective cooling that sets in after 10:00 LT. The MXL+RSAD model run, including the RSL effects, performs better than the MXL+MSAD with differences of up to $1 \mathrm{~K}$. Furthermore, the sensitivity analysis performed by varying $L_{c}$ and $\beta$ (section 3.2.2) shows that $\theta$ differs by up to almost $1 \mathrm{~K}$ at 13:00 LT for the selected sensitivity ranges (Fig. 3.8b). For the same time, the sensible heat flux ranges between 302 and $306 \mathrm{~W} \mathrm{~m}^{-2}$ (or less than $2 \%$ with respect to the case study value at 13:00 LT).

We find a slightly larger disagreement in the results for observed and modelled specific humidity at canopy-top level (up to $0.5 \mathrm{~g} \mathrm{~kg}^{-1}$, or around $5 \%$ with respect to the observed values). An interesting feature of the observations is the small difference in the magnitude (no greater than $0.5 \mathrm{~g} \mathrm{~kg}^{-1}$ ) between $29 \mathrm{~m}$ above ground (Fig. 3.4c) and canopy top (Fig. 3.9a), but we were not able to explicitly explain this small difference in $q$ between these two levels. Like the potential temperature, $q$ is sensitive to $L_{c}$ and $\beta$ at 13:00 LT, with $q$ ranging from $10.3 \mathrm{~g} \mathrm{~kg}^{-1}$ under unstable conditions to $9.0 \mathrm{~g} \mathrm{~kg}-1$ under weakly stable conditions. The maximum variations in LE for different $L_{c}$ and $\beta$ is around $34 \mathrm{~W} \mathrm{~m}^{-2}$, or around $9 \%$ with respect to the case study value at 13:00 LT $\left(362 \mathrm{~W} \mathrm{~m}^{-2}\right)$. 

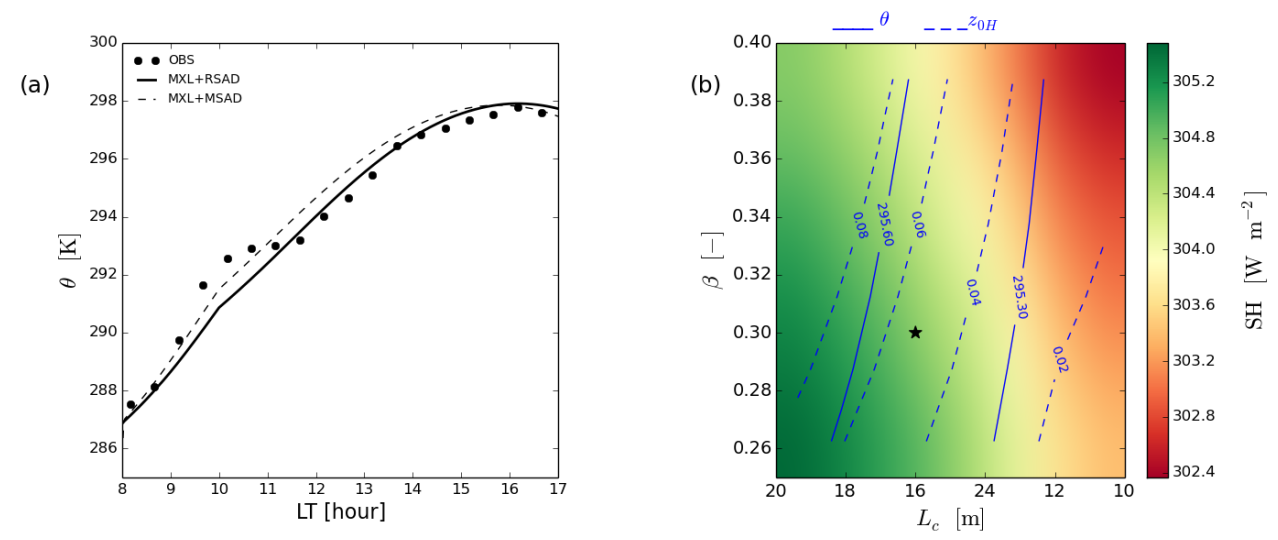

Figure 3.8: (a) Temporal evolution of the observed versus modelled potential temperature, $\theta$, with and without the RSL effects at canopy-top level. (b) Effects of $\beta$ and $L_{c}$ on sensible heat flux (SH), $\theta$ and roughness length for heat $\left(z_{0 H}\right)$ at 13:00 LT. The black asterisk indicates the conditions and the results of the case study of 27 May 2007.

Finally, in the range of $L_{c}$ and $\beta$ investigated, we found that the effective displacement height $\left(d_{t}\right)$ can range from less than $1 \mathrm{~m}$ to up to $3 \mathrm{~m}$ meters (Eq. 3.7, Fig. 3.9b). This significantly affects the roughness lengths for momentum and scalars, since $z_{0 M}$ and $z_{0 \varphi}$ are directrly dependent on $d_{t}$ and stability (Harman and Finnigan 2007; 2008; Zilitinkevich et al. 2008). These variations in the displacement height and the roughness lengths (Fig. 3.6d and Fig. 3.8b) are the cause of the variations in the surface fluxes (e.g. $2 \%$ variation in $\mathrm{SH}$ and $9 \%$ variation in LE).

\subsection{Discussion}

The interpretation of the CHATS height-dependent observations, employing a numerical model that integrates various spatial-temporal scales relevant within the CBL, reveals that the diurnal variability of the state variables above the orchard canopy is highly dependent on the contributions of local and non-local effects. Local effects are related to the land-canopy-atmosphere exchange of momentum and energy, while the non-local effects are either driven by boundarylayer dynamics, such as entrainment, or by mesoscale phenomena, such as subsidence and/or horizontal advection.

At meso-scales, as described by Hayes et al (1989), Zaremba and Carroll (1999), Bianco et al (Bianco2011) and Mayor (2011) the CHATS site is strongly influenced by various interacting mesoscale flows such as marine fronts and mountain-valley flows. Since this study focuses on convective conditions, and following the classification suggested by Zaremba and Carroll (1999, Table 3 and Fig. 4b,c), we studied two cases characterized by different mesoscale circulations: $i$ ) 

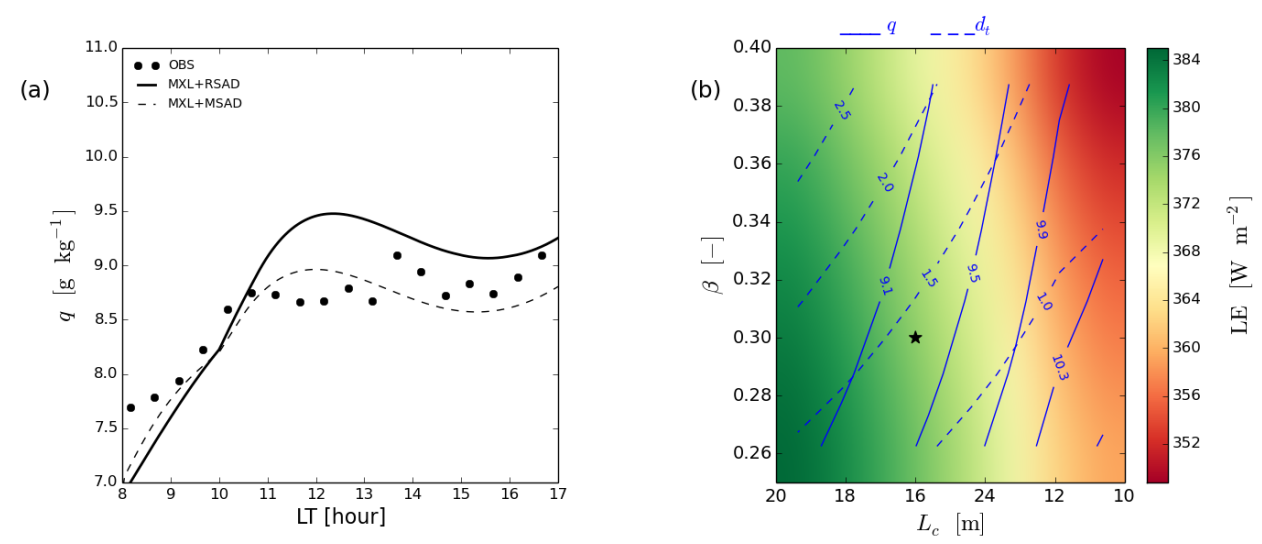

Figure 3.9: (a) Temporal evolution of the observed versus modelled specific humidity, $q$, with and without the RSL effects at canopy-top level. (b) Effects of stability dependent $\beta$ and $L_{c}$ at canopy top on sensible heat flux (LE), $q$ and the effective displacement height $\left(d_{t}\right)$ at 13:00 LT. The black asterisk indicates the conditions and the results of the case study (27 May 2007).

a case with southerly dominant winds and $i$ ) a day with northerly winds that veer south at around noon. In both cases, the impact of the marine mesoscale flow coming from the San Francisco Bay area (e.g. Zaremba and Carroll 1999, Fig. 7b,c) leads to a sudden decrease in the rate of growth of the boundary-layer height (Fig. 3.4a). This yielded an almost constant $h$ at around $500 \mathrm{~m}$ for the case of 27 May (Fig. 3.4a) and around $650 \mathrm{~m}$ on 31 May (see supplementary material).

In the absence of detailed observations of the temporal evolution at the entrainment zone, we are able to provide only first-order estimates of the large-scale effects relevant to our cases and discuss their impacts on the budgets of potential temperature and specific humidity (Fig. 3.10). The budgets of potential temperature (Fig. 3.10a) and specific humidly (Fig. 3.10b) enable us to quantify the relevance of non-local versus local processes. Overall, surface and entrainment are the main contributors to the variability of the potential temperature and specific humidity. Besides these, the advective cooling and moistening process has a relatively large impact on the corresponding budgets after 10:00 LT, when advection is employed to capture the observed diurnal evolution of $\theta$ and $q$ (Fig. 3.4b,c). The negative $\theta$-tendency and positive $q$-tendency due to advection in this analysis (the green solid lines in Fig. 3.10) corroborate the drop in air temperature and increase in moisture which were observed over the Sacramento Valley flow, characterised by southerly winds (Zaremba and Carroll 1999, Bianco et al. 2011).

Focusing now on the surface conditions, and on canopy scales, the representation of the RSL has a large impact on the drag coefficients and mean gradients of the thermodynamic variables within the RSL, and to a lesser extent to the surface fluxes. Our findings are in agreement with those of Physick and Garratt (1995) and Maurer et al. (2013), and raise a potential paradox. Even 

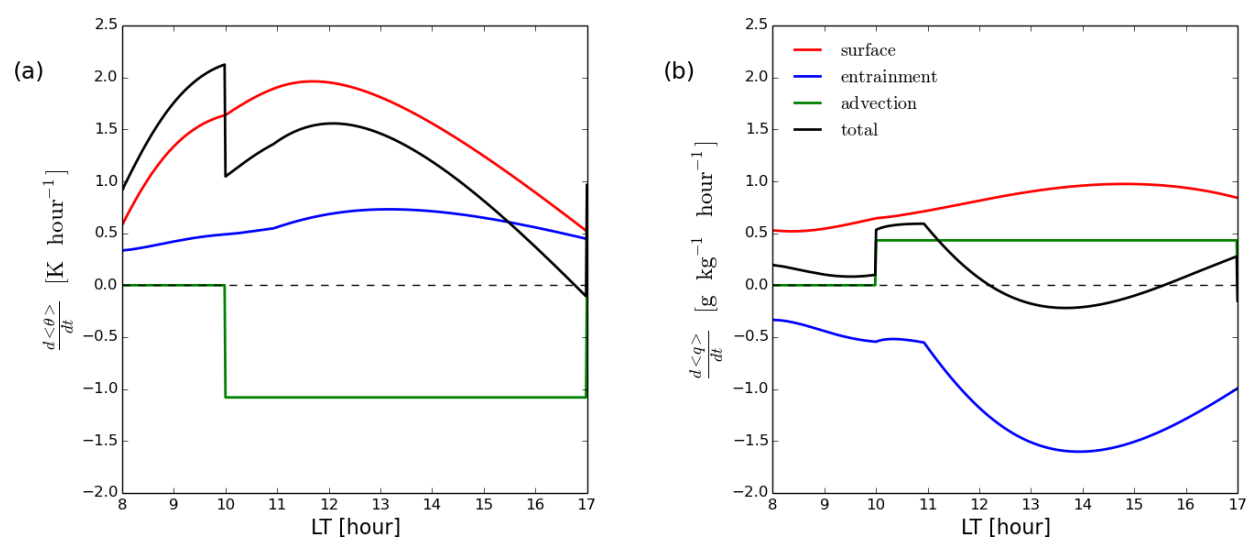

Figure 3.10: (a) Temporal evolution of the observed versus modelled specific humidity, $q$, with and without the RSL effects at canopy-top level. (b) Effects of stability dependent $\beta$ and $L_{c}$ at canopy top on sensible heat flux (LE), $q$ and the effective displacement height $\left(d_{t}\right)$ at 13:00 LT. The black asterisk indicates the conditions and the results of the case study (27 May 2007).

though surface fluxes inferred from gradient observations just above the canopy are affected by roughness sublayer effects (Mölder et al. 1999; De Ridder 2010), the actual (modelled) fluxes are only insignificantly different for the standard conditions ( $L_{c}=16 \mathrm{~m}$ and $\left.\beta=0.3\right)$. This is due to the parameterization of the surface fluxes depending on both the drag coefficient and the difference of the mean variable (Eqs. 3.2-3.3). As we showed (e.g. Fig. 3.6a,b), both are strongly affected by the effects of RSL correction, but they compensate each other. The momentum flux is more sensitive to the variations in $L_{c}$ and $\beta$ than the sensible and latent heat fluxes. This is due to the boundary condition that relates the surface value to the atmospheric value. While a Dirichlet boundary condition is applied to momentum (no wind at roughness height for standard MOST), a Neumann boundary condition is required for potential temperature and specific humidity. $T_{s}$ depends on the SEB (Sect. 3.2.2) and is determined as a function of the radiation, soil heat flux, $\theta\left(z_{r}\right), q\left(z_{r}\right), r_{a H}$ and $r_{s}$ (see e.g. van Heerwaarden et al. 2009). Since $\theta\left(z_{r}\right), q\left(z_{r}\right), r_{a H}$ and $r_{s}$ are altered by the RSL, $T_{s}$ and $q_{s}$ are affected as well, resulting in minor variations in the mean gradient (see also Harman 2012, Fig. 4a,b) and therefore smaller variation in the surface flux (Eq. 3.2). This is the reason why we found larger fluctuation in the friction velocity $(25 \%)$ for different RSL scales $\left(\beta\right.$ and $L_{c}$ ), compared to the much smaller variations in SH (2\%) and LE (9 $\%)$. 


\subsection{Conclusions}

By combining observations, collected at different heights above a walnut orchard canopy during the Canopy Horizontal Array Study (CHATS), with model experiments performed incorporating a land-vegetation-atmosphere model, we investigated the contributions of canopy and large-scale atmospheric forcings on the diurnal variability of boundary-layer height, the evolution of mixedlayer properties and of canopy-atmosphere exchange of momentum, potential temperature and specific humidity. We selected a representative day with southerly wind conditions for our study to maximize the effects of the canopy fetch and compared it with another day ( wind veering from northerly to southerly) characterized by less fetch influence. We pay particular attention to determine the sensitivity of the surface fluxes and the boundary-layer evolution to changes in the canopy adjustment length scale, $L_{c}$, and the ratio between the friction velocity and the wind speed at the canopy top, $\beta$, which are relevant scales within the roughness sublayer.

On the bases of our findings, we reach the following conclusions:

- The investigated CHATS convective boundary layers are strongly affected by large-scale processes such as advective cooling, subsidence and entrainment of dry and warm air from the free troposphere. Quantifying these large scaling forcings by using the observations, the coupled soil-vegetation-atmosphere modelling system satisfactorily represents the surface fluxes and convective boundary-layer dynamics at the CHATS site.

- In our modelling framework, and in general in the coupled land-atmosphere models, the representation of the surface fluxes is locked and controlled by the boundary conditions. The sensible and latent heat fluxes are bounded by the surface available energy, and the momentum flux is constrained by the pressure gradient and the entrainment of momentum, the latter dependent on the boundary-layer growth. In consequence, adding a roughnesssublayer representation in the surface scheme of the model alters the partitioning of the surface fluxes (e.g. sensible and latent heat) through the altered roughness length and displacement height. Specifically for our case studies, the canopy?s impact on convective boundary-layer dynamics is relatively minor, due to its small effect on modelled surface fluxes and the bulk boundary-layer properties well above the canopy $\left(z>2 h_{c}\right)$. The tall canopy however strongly affects the mean gradients and transfer coefficients within the roughness sublayer. Thus, considering the roughness sublayer parameterization is important when comparing observations and large-scale model outputs of the mean quantities near and just above the canopy.

- The sensitivity analysis on roughness sublayer scales, analysed through changes in $L_{c}$ and $\beta$, and their diabatic stability dependence, led to changes in the friction velocity (up to 25 $\%$ ) and smaller variations in the sensible and latent heat fluxes (2\% and $9 \%$ respectively), leading to changes in the boundary layer height of up to $6 \%$. 
- Changes in $\beta$ significantly impact the surface drag contribution to the mixed-layer momentum budget (up to $25 \%$ variation for the given range of $\beta$ ). The altered surface momentum due to changes in $\beta$ is compensated by changes in geostrophic forcing and entrainment resulting in a similar total momentum tendency.

- When interpreting the CHATS measurements above the canopy, the mesoscale advective processes or subsidence play an important role in determining the convective boundarylayer dynamics. Analysis of the bulk potential temperature and specific humidity budgets showed that the influence of the advection can be around one fourth of the total potential temperature budgets. 


\section{A Appendix: Mixed-layer model initial and boundary conditions}

Table 3A.1: Initial and boundary conditions for model runs of 27 May 2007 (147 DOY) for the CHATS experiment.

\begin{tabular}{|c|c|c|}
\hline Variable & Description and unit & Value \\
\hline & MXL model run & \\
\hline$t$ & time domain $[\mathrm{s}]$ & 32400 \\
\hline$d t$ & time step $[\mathrm{s}]$ & 10 \\
\hline lat & latitude $[\mathrm{deg}]$ & $38.45 \mathrm{~N}$ \\
\hline lon & longitude $[\mathrm{deg}]$ & $-121.8 \mathrm{E}$ \\
\hline DOY & day of the year & 147 \\
\hline \multirow[t]{2}{*}{ hour } & starting time of the model run [LT] & 147 \\
\hline & Boundary-layer dynamics & \\
\hline$P_{0}$ & surface pressure $[\mathrm{Pa}]$ & 102900 \\
\hline$h_{0}$ & boundary-layer height at 08:00LT [m] & 350 \\
\hline$w_{s}$ & large-scale vertical velocity $\left[\mathrm{m} \mathrm{s}^{-1}\right]$ & $5 \times 10^{-5}$ \\
\hline$<\theta_{0}>$ & initial mixed-layer potential temperature $[\mathrm{K}]$ & 286.5 \\
\hline$\Delta \theta_{0}$ & initial temperature jump at the entrainment zone $[\mathrm{K}]$ & 1.5 \\
\hline$\gamma_{\theta}$ & potential temperature lapse rate in free troposphere $\left[\mathrm{K} \mathrm{m}^{-1}\right]$ & 0.017 \\
\hline $\operatorname{Adv}_{\theta}$ & advection of heat $\left[\mathrm{K} \mathrm{s}^{-1}\right]$ (hour $\left.>10: 00 \mathrm{LT}\right)$ & $0\left(-3 \times 10^{-4}\right)$ \\
\hline$<q_{0}>$ & initial mixed-layer specific humidity $\left[\mathrm{kg} \mathrm{kg}^{-1}\right]$ & $7.6 \times 10^{-3}$ \\
\hline$\Delta q_{0}$ & $\begin{array}{l}\text { initial specific humidity jump at the entrainment zone }[\mathrm{kg} \\
\left.\mathrm{kg}^{-1}\right]\end{array}$ & $2 \times 10^{-4}$ \\
\hline$\gamma_{q}$ & $\begin{array}{l}\text { specific humidity lapse rate in free troposphere }\left[\mathrm{kg} \mathrm{kg}^{-1} \mathrm{~m}^{-1}\right] \\
(h>500 \mathrm{~m})\end{array}$ & $0\left(10^{-4}\right)$ \\
\hline $\operatorname{Adv}_{q}$ & advection of moisture $\left[\mathrm{kg} \mathrm{kg}^{-1} \mathrm{~s}^{-1}\right]$ (hour $>10: 00 \mathrm{LT}$ ) & $0\left(10^{-4}\right)$ \\
\hline$<u_{0}>$ & initial longitudinal mixed-layer wind speed $\left[\mathrm{m} \mathrm{s}^{-1}\right]$ & 0 \\
\hline$<v_{0}>$ & initial lateral mixed-layer wind speed $\left[\mathrm{m} \mathrm{s}^{-1}\right]$ & 1.5 \\
\hline$u_{g}$ & geostrophic longitudinal wind speed $\left[\mathrm{m} \mathrm{s}^{-1}\right]$ & 0 \\
\hline$v_{g}$ & geostrophic lateral wind speed $\left[\mathrm{m} \mathrm{s}^{-1}\right]$ & 4 \\
\hline$\gamma_{u}$ & free atmosphere wind speed (longitudinal) lapse rate $\left[\mathrm{s}^{-1}\right]$ & 0.03 \\
\hline \multirow[t]{2}{*}{$\gamma_{v}$} & free atmosphere wind speed (lateral) lapse rate $\left[\mathrm{s}^{-1}\right]$ & 0 \\
\hline & Roughness sublayer & \\
\hline$z_{0 M}$ & initial roughness length for momentum [m] & 0.7 \\
\hline
\end{tabular}


$\begin{array}{llr}z_{0 \varphi} & \text { initial roughness length for heat and moisture [m] } & 0.095 \\ L_{c} & \text { roughness-sublayer penetration depth [m] } & 16 \\ \beta & \text { roughness sublayer scaling parameter [-] } & 0.3\end{array}$

\section{Soil and vegetation}

$\begin{array}{lll}c c & \text { cloud cover [-] } & 0.07\end{array}$

$\begin{array}{lll}\alpha & \text { albedo [-] } & 0.15\end{array}$

$T_{s} \quad$ initial surface temperature $[\mathrm{K}] \quad 291$

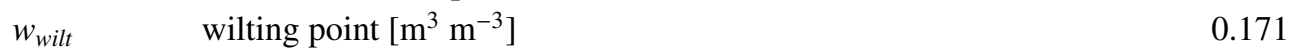

$w_{2} \quad$ volumetric water content deeper soil layer $\left[\mathrm{m}^{3} \mathrm{~m}^{-3}\right] \quad 0.26$

$w_{g} \quad$ volumetric water content top sloil layer $\left[\mathrm{m}^{3} \mathrm{~m}^{-3}\right] \quad 0.26$

$w_{f c} \quad$ volumetric water content field capacity $\left[\mathrm{m}^{3} \mathrm{~m}^{-3}\right] \quad 0.323$

$\begin{array}{lll}w_{\text {sat }} & \text { saturated volumetric water content }\left[\mathrm{m}^{3} \mathrm{~m}^{-3}\right] & 0.472\end{array}$

$\begin{array}{lll}C_{1 \text { sat }} & \text { coefficient force term moisture [-] } & 0.132\end{array}$

$\begin{array}{lll}C_{2 \text { ref }} & \text { coefficient restore term moisture [-] } & 1.8\end{array}$

$r s_{\text {min }} \quad$ minimum resistance of transpiration $\left[\mathrm{s} \mathrm{m}^{-1}\right] \quad 110$

$r s_{\text {soilmin }} \quad$ minimum resistance of soil transpiration $\left[\mathrm{s} \mathrm{m}^{-1}\right] \quad 50$

LAI leaf area index $\left[\mathrm{m}^{2} \mathrm{~m}^{-2}\right] \quad 2.5$

$\begin{array}{ll}c_{\text {veg }} \quad \text { vegetation fraction [-] } & 0.9\end{array}$

$T_{\text {soil }} \quad$ initial temperature top soil layer [K] 290

$\begin{array}{lll}T_{2} & \text { temperature deeper soil layer }[\mathrm{K}] & 289\end{array}$

$\Lambda \quad$ thermal conuctivity skin layer divided by depth [W $\left.\mathrm{m}^{-2} \mathrm{~K}^{-1}\right]$

$C G_{\text {sat }} \quad$ saturated soil conductivity for heat $\left[\mathrm{W} \mathrm{m}^{-2} \mathrm{~K}^{-1}\right] \quad 3.6 \times 10^{-6}$

Table 3A.2: Initial and boundary conditions for model runs of 31 May 2007 (151 DOY) for the CHATS experiment (similar to Table 3A.1; here, only the differences are presented).

\begin{tabular}{|c|c|c|}
\hline Variable & Description and unit & Value \\
\hline & Boundary-layer dynamics & \\
\hline$h_{0}$ & boundary-layer height at 08:00LT [m] & 250 \\
\hline$w_{s}$ & large-scale vertical velocity $\left[\mathrm{m} \mathrm{s}^{-1}\right]$ & $3.5 \times 10^{-5}$ \\
\hline$<\theta_{0}>$ & initial mixed-layer potential temperature $[\mathrm{K}]$ & 286.5 \\
\hline$\Delta \theta_{0}$ & initial temperature jump at the entrainment zone $[\mathrm{K}]$ & 1.0 \\
\hline$\gamma_{\theta}$ & potential temperature lapse rate in free troposphere $\left[\mathrm{K} \mathrm{m}^{-1}\right]$ & 0.017 \\
\hline $\operatorname{Adv}_{\theta}$ & advection of heat $\left[\mathrm{K} \mathrm{s}^{-1}\right]$ (hour $\left.>10: 00 \mathrm{LT}\right)$ & $0\left(-1.3 \times 10^{-4}\right)$ \\
\hline$<q_{0}>$ & initial mixed-layer specific humidity $\left[\mathrm{kg} \mathrm{kg}^{-1}\right]$ & $7.6 \times 10^{-3}$ \\
\hline
\end{tabular}




$\begin{array}{llr}\Delta q_{0} & \begin{array}{l}\text { initial specific humidity jump at the entrainment zone }[\mathrm{kg} \\ \left.\mathrm{kg}^{-1}\right]\end{array} & 2 \times 10^{-4} \\ \gamma_{q} & \text { specific humidity lapse rate in free troposphere }\left[\mathrm{kg} \mathrm{kg}^{-1} \mathrm{~m}^{-1}\right] & 0\left(8 \times 10^{-5}\right) \\ & (h>500 \mathrm{~m}) & 1 \\ <u_{0}> & \text { initial longitudinal mixed-layer wind speed }\left[\mathrm{m} \mathrm{s}^{-1}\right] & -2.5 \\ <v_{0}> & \text { initial lateral mixed-layer wind speed }\left[\mathrm{m} \mathrm{s}^{-1}\right] & 0 \\ u_{g} & \text { geostrophic longitudinal wind speed }\left[\mathrm{m} \mathrm{s}^{-1}\right] & -2(1.5) \\ v_{g} & \left.\text { geostrophic lateral wind speed [m s} \mathrm{m}^{-1}\right] & 0.08 \\ \gamma_{u} & \text { free atmosphere wind speed (longitudinal) lapse rate }\left[\mathrm{s}^{-1}\right] & 0 \\ \gamma_{v} & \text { free atmosphere wind speed (lateral) lapse rate }\left[\mathrm{s}^{-1}\right] & \end{array}$

Note that the values in the round brackets represent the prescribed changes in the model initialization depending on the boundary layer height (for $\gamma_{\theta} \theta$ and $\gamma_{y}$ ) (if $h>500 \mathrm{~m}$ ) and the time after 10:00 LT (for the advection).

\section{B Appendix: Momentum budget}

Assuming that in the free troposphere the wind is in balance (equilibrium) between the pressure gradients and Coriolis force, the budgets of the mixed-layer wind components are expressed by the following equations:

$$
\begin{aligned}
& \frac{d<u>}{d t}=\frac{\left(\overline{w^{\prime} u^{\prime}}\right)_{s}-\left(\overline{w^{\prime} u^{\prime}}\right)_{e}}{h}-f_{c}\left(<v>-v_{g}\right), \\
& \frac{d<v>}{d t}=\frac{\left(\overline{w^{\prime} v^{\prime}}\right)_{s}-\left(\overline{w^{\prime} v^{\prime}}\right)_{e}}{h}+f_{c}\left(<u>-u_{g}\right) .
\end{aligned}
$$

The modulus of the wind speed components then is:

$$
<|U|>=\sqrt{\left.<u\rangle^{2}+<v\right\rangle^{2}} .
$$

Combing the Eqs. (3B.1-3B.3), results in:

$$
\begin{aligned}
\frac{d<U>}{d t} & =\frac{1}{<|U|>}\left\{\left[\left(<u>\frac{\left(\overline{w^{\prime} u^{\prime}}\right)_{s}}{h}+<v>\frac{\left(\overline{w^{\prime} v^{\prime}}\right)_{s}}{h}\right)-\left(<u>\frac{\left(\overline{w^{\prime} u^{\prime}}\right)_{e}}{h}+<v>\frac{\left(\overline{w^{\prime} v^{\prime}}\right)_{e}}{h}\right)\right]+\right. \\
& \left.+f_{c}\left[<v>\left(<u>-u_{g}\right)-<u>\left(<v>-v_{g}\right)\right]\right\},
\end{aligned}
$$


where:

$\frac{d<U>}{d t}$ is the total wind speed tendency;

$\frac{1}{<|U|>}\left[\left(<u>\frac{\left(\overline{w^{\prime} u^{\prime}}\right)_{s}}{h}+<v>\frac{\left(\overline{w^{\prime} v^{\prime}}\right)_{s}}{h}\right)\right]$ is the surface forcing (due to surface stress and canopy drag);

$\frac{1}{<|U|>}\left[-\left(<u>\frac{\left(\overline{w^{\prime} u^{\prime}}\right)_{e}}{h}+<v>\frac{\left(\overline{w^{\prime} v^{\prime}}\right)_{e}}{h}\right)\right]$ is the entrainment forcing;

$\frac{1}{<|U|>} f_{c}\left[<v>\left(<u>-u_{g}\right)-<u>\left(<v>-v_{g}\right)\right]$ is the geostrophic forcing. 


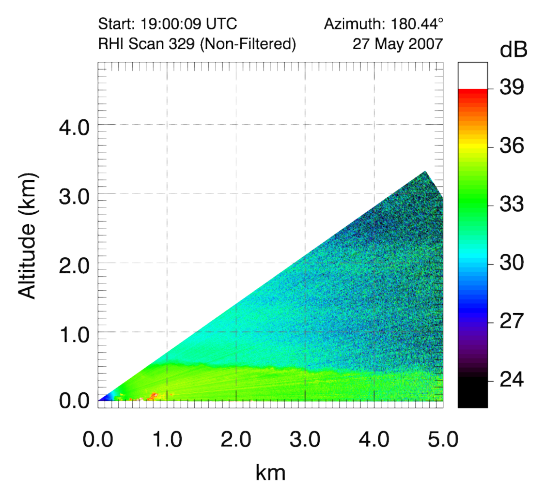

Figure 3C.1: REAL backscatter data (in dB) on 27 May 2007 at 19:00:09 UTC (12:00:09 LT), plotted in height (y-axis) and distance from the LIDAR (x-axis), and for several elevations.

\section{C Appendix: Estimating the boundary-layer height from LIDAR data}

To calculate the boundary-layer height observed during the CHATS experiment (Patton et al. 2011), we make use of the NCAR Raman-shifted Eye-Safe Aerosol Lidar (REAL) backscatter signal data. The application of REAL at CHATS experiment, its purpose and operational characteristics in the campaign are presented in Patton et al. (2011). Here we describe the algorithm employed to calculate the boundary-layer height using the above mentioned data.

The automatic method to calculate the boundary-layer height, which is presented in this document, is based on the method of the height of the maximal vertical gradient of the aerosol backscatter profiles (Endlich et al. 1979). The steps of the algorithm are presented below.

- Visualisation of the two-dimensional (elevation-height) backscatter data

The data is stored as two-dimensional arrays (distance from the LIDAR, elevation). Each of the 175 arrays (175 elevations) for certain time-period (around 11 seconds) has 4200 signal cells with equidistant spacing of 1.5 meter. To construct profiles of the signal in height and distance from the LIDAR, the data is transferred from polar coordinates to Cartesian coordinate system (Fig. 3C.1).

- Retrieving the backscatter vertical profiles

Two methods of retrieving the profiles from the raw backscatter data are used and compared: i) using a single profile at 50 per cent of the distance (i.e. at $2500 \mathrm{~m}$ ), or ii) calculating an average profile from the profiles within a certain range. We averaged all the profiles between the 1000th and 2000th cell (in total 1000 profiles). Figure 3C. 2 shows a typical example using both methods. 


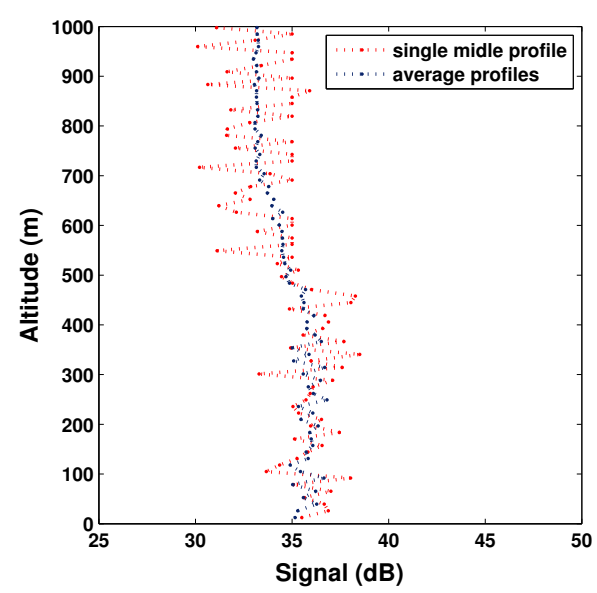

Figure 3C.2: Retrieved profiles from the raw data at the same date and time as presented in Fig. 3B. 1

The single-half-way profile retrieved from i) shows larger fluctuations than the averaged profile. However, in order to eliminate and filter out short-range fluctuations, both aerosol backscatter profiles are smoothed in the next step.

- Smoothening the vertical profile

A moving average window, using five data points (three data points under stable conditions), is used to filter the large fluctuations in the profiles (Fig. 3C.3).

- Calculating the derivative of the backscatter (vertical)

The smoothed profiles of the raw backscatter signal indicate the layer of the maximal gradient. In our example, the maximum gradient height is between 400 and $600 \mathrm{~m}$. To calculate more precisely the location of the maximal gradient, we calculate the vertical derivatives of the signal elements in the smoothed profile array. More specifically, we take the derivate of every fifth element in the array, with respect to the previous fifth element, rather than taking the derivative of every second element along the array. This gives us a profile of the derivatives (vertical) of the smoothed backscatter profile with peaks at half of the distance between five elements within the array (Fig. 3C.4a). We do this in order to prevent erroneous maximal gradients from the strong and sharp local differences (gradients) in the data.

- Finding the height of the maximal gradient (negative) in the smoothed profile

The height of the largest negative gradient is the height of the boundary layer for the corresponding profile (at that moment in time). Since we do not consider the positive gradients, we set them 

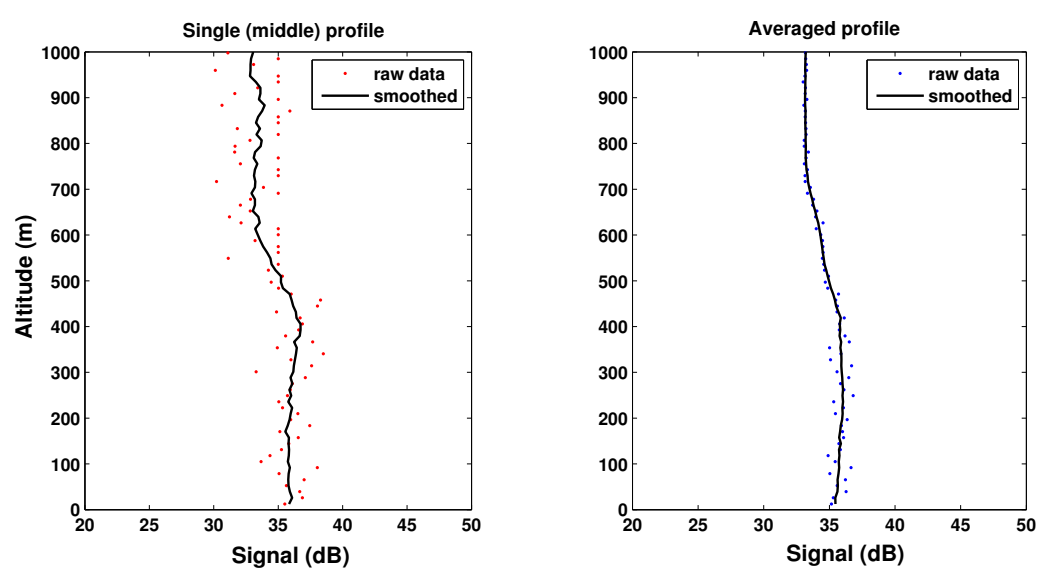

Figure 3C.3: Smoothing the profiles of the backscatter signal (dB) using the moving-average method taking eight data-points average in the smoothing, for both, single (left) and averaged (right) profiles. Note that the profiles are the same as presented in Fig. 3C.2, here divided into two panels.

all equal to zero (Fig. 3C.4b). We then find the negative maximum in the derivative profile (Fig. 3C.4b), and the height where this maximum occurs (BL-height). Finally, in order to check if the height of the maximal gradient is consistent with the negative slope in the smoothed backscatter profile, we plot the line of the BL-height and visualize where it crosses the profile (Fig. 3C.4c). The latter is visual confirmation (check) of the found BL-height.

- Plotting the heights of the maximal gradients (BL-height) in time (evolution)

In order to find the temporal evolution of the BL-height, we repeat the above procedure for all the lidar-scans in time. Here, we present the diurnal evolution of the BL-height for the 27th of May 2007 (147th Julian day) (Fig. 3C.5). We take this example because it was characterized by southerly winds, ensuring the largest fetch above the CHATS orchard.

Note that this method is valid for standard (typical) profiles of the backscatter. It is sensitive to atypical profiles (e.g. Fig. 3C.6), disrupted by advection effects that bring layers of aerosols with different characteristics, or for cases with a strong residual layer (e.g. morning-noon transition). Figure 3C.5 shows an example in which the method described above has limitations. Figure 3C.6 shows the aerosol concentration in distance and height. There are two heights at which the layers exhibit a sharp negative slope. The first is within the first $100 \mathrm{~m}$ and the second is between 300 and $400 \mathrm{~m}$. Since from this profile it is difficult to conclude where the BL-height should be, the method deals with this problem by choosing one of the two large maxima, which may not represent the true BL-height. This is illustrated in Fig. 3C.6; between 13:00 - 15:00 UTC (06:00 - 08:00 Local Time) we evaluated very high and very low values for the BL-height. 

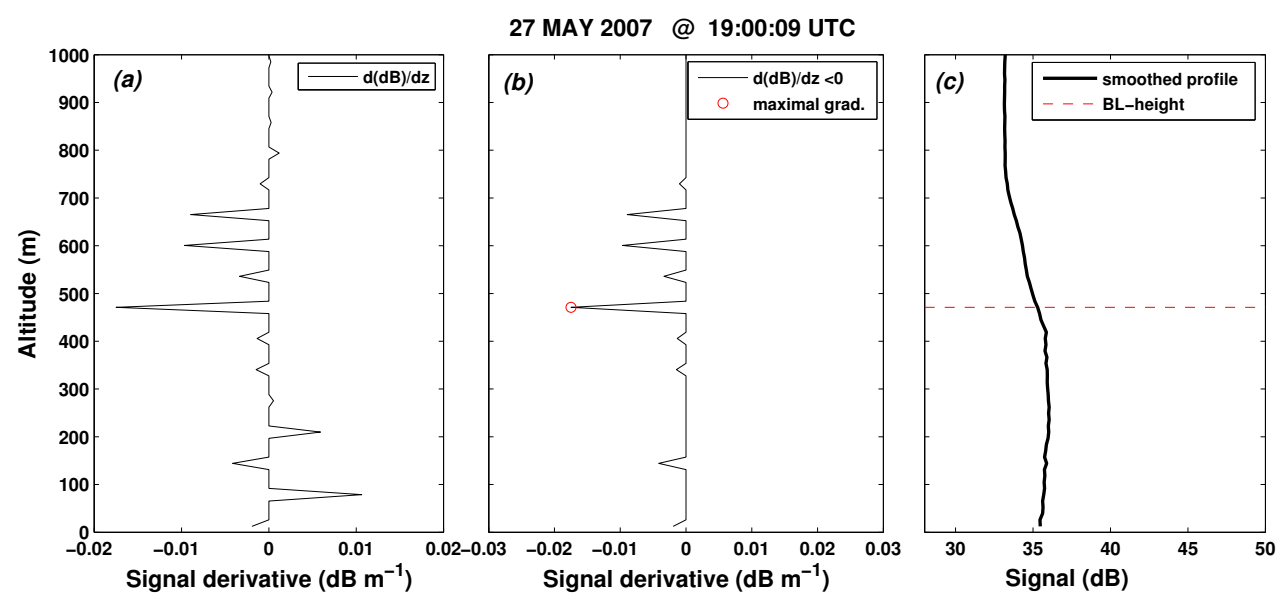

Figure 3C.4: (a) Profile of the vertical derivative of the smoothed backscatter data; (b) the maximal negative gradient; (c) the boundary-layer height based on the smoothed profile for 27 May (19:00:09 UTC or 12:00:09 LT).

In this case, the aerosol backscatter does not exhibit a regular profile, so the method to calculate the BL-height can lead to erroneous results. One possible solution would be to take the average height of both maxima, including the large fluctuation in the calculation as uncertainty (not shown). 


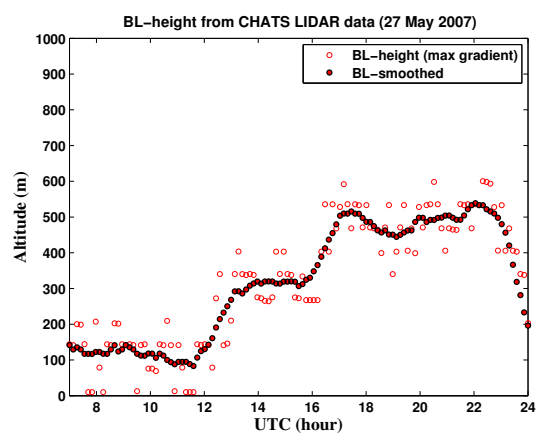

Figure 3C.5: Temporal evolution of the boundary-layer height calculated from the CHATS LIDAR backscatter data for 27 May 2007.

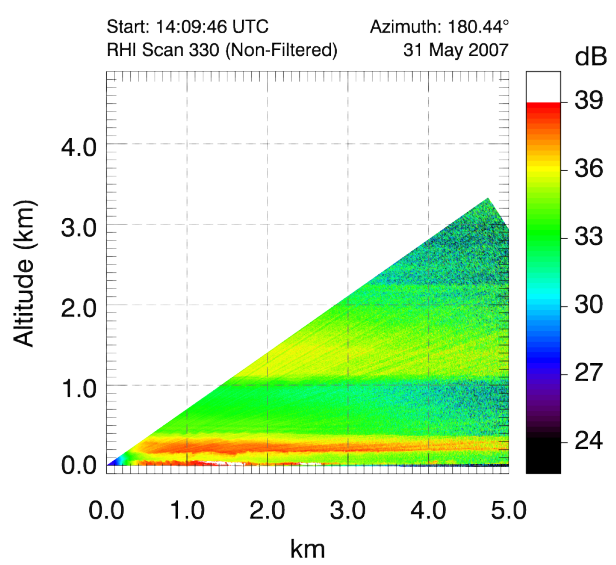

Figure 3C.6: REAL backscatter data (in dB) on 31 May 2007 at 14:00:46 UTC (07:09:46 LT), plotted in height (y-axis) and distance from the LIDAR (x-axis), and for several elevations. 


\title{
4
}

\section{Combined effects of deposition, entrainment and chemistry on the ozone budget over an orchard canopy}

\begin{abstract}
The interaction between a tall vegetative canopy and the atmosphere can influence the ozone $\left(\mathrm{O}_{3}\right)$ budget due to changes in the distribution of sources and sinks of $\mathrm{O}_{3}$ and the canopy's influence on boundary layer dynamics. To investigate these complex interconnected processes, first a comprehensive set of measurements is required. Here, we make intensive use of the observations collected at a mature walnut orchard during the Canopy Horizontal Array Turbulence Study (CHATS) near Dixon, California. The CHATS dataset consists of detailed micrometeorology and gas concentration profiles taken at different heights along a $30 \mathrm{~m}$ tower during spring, 2007. This dataset gives the possibility to analyze in detail the different behaviour of state variables and reactants in and above the canopy. More specifically, we calculate the diurnal variability of the vertical fluxes of ozone and the impact of dry deposition, entrainment, advection and chemistry on the $\mathrm{O}_{3}$-budget. As no fluxes of gases were directly observed during CHATS, dry deposition rates were calculated from vertical fluxes derived from the observed concentrations within and above the canopy. We calculated fluxes of $\mathrm{O}_{3}, \mathrm{NO}, \mathrm{NO}_{2}$ and their
\end{abstract}

This Chapter is a manuscript in preparation with (co)authors: Jessica Brown (Wageningen University), Maarten Krol (Wageningen University), Thomas Karl (University of Innsbruck), Arnold Moene (Wageningen University), Huug Ouwersloot (Max-Planck Institute for Chemistry) and Jordi Vilà-Guerau de Arellano (Wageningen University). 
deposition rates using four different methods above and below the canopy top. We apply a method which explicitly includes the effects of tall canopy on the flux-gradient relationships above the canopy, as well as a method considering non-local transport processes within the canopy. Following the calculation of deposition rates, a zero-dimensional mixed-layer model was used to study the diurnal variability of $\mathrm{O}_{3}$ in the mixed layer above the canopy at CHATS. This mixed layer model integrates aspects of the $\mathrm{O}_{3}$ budget: deposition, entrainment, advection and ozone production due to chemistry. We found that the omitting the roughness sublayer effects in the ozone flux retrieval may lead to $50 \%$ smaller diagnosed deposition velocities. This may further ledd to a $16 \%$ (diurnal average) overestimation in the mixed layer ozone budget and up to $9 \%$ variation in $\mathrm{NO}_{x}\left(=\mathrm{NO}+\mathrm{NO}_{2}\right)$ budget. Reproduction of the observed $\mathrm{NO}_{2} / \mathrm{NO}$ ratio and photostationary state, for the studied case, required high levels of VOCs combined with positive advection of $\mathrm{NO}_{x}$, and negative advection of ozone over CHATS. The ozone budget closure analysis implied a strong influence of chemistry and horizontal advection processes on the diurnal ozone variation at CHATS. Due to a lack of VOC measurements however, a more detailed quantification could not be provided. The analysis however showed that the effective exchange of reactants (e.g. ozone) in the roughness sublayer (quantified by the deposition velocity of ozone), is strongly affected by the presence of the high vegetation.

\subsection{Introduction}

The contribution of both chemical and dynamic processes to the total budget of these reactive compounds in the convective boundary layer can be often of similar order (e.g. van Stratum et al. 2012) and therefore should be studied in an integrated manner (Vilà-Guerau de Arellano 2003). Moreover, the emission or deposition of the chemically reactive compounds at the Earth's surface can be of a similar magnitude to the entrainment at the boundary-layer top (Ganzeveld et al. 2008; Vilà-Guerau de Arellano et al. 2011). The presence of tall vegetation complicates the picture further, because high vegetation can alter the surface energy balance (e.g. Foken 2008b), the emission of volatile organic compounds (e.g. Karl et al. 2004), and turbulence properties within the boundary layer (Harman 2012). In addition, the vertical distribution of certain species in the convective boundary layer can be altered by conditions such as surface heterogeneity (Ouwersloot et al. 2012). As such, the presence of a tall canopy and the changes in its conditions due to factors such as drought stress may play an important role in determining the budget of reactive species. In this study, we aim to investigate the combined effect of dynamics and chemistry on the diurnal evolution of ozone $\left(\mathrm{O}_{3}\right)$ within the boundary layer above a relatively tall and sparse vegetative canopy. More specifically, we seek to investigate the influence of high vegetation on vertical turbulent fluxes of reactants within and above the canopy and their effect on the $\mathrm{O}_{3}$ budget.

Ozone is a principal component of photochemical smog, and can cause damage to surfaces, plants and human health (Griffin et al. 2007). $\mathrm{O}_{3}$ is produced by photochemical reactions of $\mathrm{NO}_{x}$ and volatile organic compound (VOCs) (Fuentes et al. 2000; Jacobson 2005; Seinfeld and Pandis 2016). VOCs (e.g. isoprene, terpenes) are commonly emitted by plants as a response 


\subsection{INTRODUCTION}

to stress factors such as temperature or herbivory (Loreto and Schnitzler 2010). Apart from being an $\mathrm{O}_{3}$ precursor, rapid gas-phase reactions between VOCs and $\mathrm{O} 3$ within the canopy can quickly deplete ozone close to the surface, which leads to an apparent deposition flux (Goldstein et al. 2004). Moreover, $\mathrm{O}_{3}$ concentrations are influenced by the stomatal uptake of vegetation (Fares et al. 2012). The presence of a tall canopy not only influences the $\mathrm{O}_{3}$ budget through the emission of VOCs and deposition of $\mathrm{O}_{3}$ but also changes the turbulent mixing. This is due to the altered turbulent mixing at the canopy-atmosphere interface (Raupach et al. 1996), which is driven by air movements characterized by sweeps and ejections (Finnigan et al. 2009; Dupont and Patton 2012a). The layer in which the turbulence structure is strongly influenced by the individual roughness elements is called the roughness sublayer (RSL). The altered turbulent mixing in the RSL leads to an enhanced turbulent transport relative to transport predicted by standard eddy diffusivity surface-layer theory (Iwata et al. 2010; Shapkalijevski et al. 2016).

To investigate the relationship between $\mathrm{O}_{3}$, plants, dynamics, and chemistry we use the observations gathered during the Canopy Horizontal Array Turbulence Study (CHATS). The CHATS campaign was undertaken in spring 2007 in central California. The aim of this campaign was to obtain a dataset that helps in understanding vegetation, atmosphere, and land surface interactions (Patton et al. 2011). The CHATS dataset includes detailed vertical profiles of micrometeorological variables and trace gas mixing ratios. As no gas fluxes were directly observed at the CHATS site, and fluxes of $\mathrm{O}_{3}$ are required in the $\mathrm{O}_{3}$ budget, we use the several methods to calculate fluxes of species within and above the canopy relevant to the $\mathrm{O}_{3}$ budget. Thus, the first objective of this study is to investigate and quantify the effects of a tall canopy on calculated gas fluxes from observed concentration profiles in and above the canopy. We calculate the fluxes of $\mathrm{O}_{3}$, $\mathrm{NO}$ and $\mathrm{NO}_{2}$ using three variations of the flux-gradient method, including one method which specifically accounts for the effects of the high vegetation on the atmospheric flow. The flux-gradient method is fundamental for observations and modeling of turbulent surface fluxes (Iwata et al. 2010). It is essential for calculating fluxes of reactive gases for which the availability of fast-response analyzers limits the use of the eddy-covariance approach (Rannik et al. 2004). Since the turbulence within the canopy often has a non-diffusive character (driven by non-local effects, (e.g. Denmead and Bradley 1985; 1987)), contrary to the assumption in the flux-gradient method, we also use the Lagrangian framework to retrieve the gas fluxes from the in-canopy concentration profiles via the inverse localized near-field theory (Raupach 1989a). Moreover, since during the CHATS campaign the orchard was irrigated, we further discuss the effects of changes in canopy conditions due to water stress on the exchange of ozone within and above the canopy.

To integrate the effects of chemistry and dynamics on the $\mathrm{O}_{3}$ budget we use a soil-vegetationatmospheric mixed-layer model (van Heerwaarden et al. 2009) coupled to a range of chemistry schemes (Vilà-Guerau de Arellano et al. 2009; Ouwersloot et al. 2012). Our objective here is to reproduce the $\mathrm{O}_{3}$ budget over the CHATS site and to quantify the contributions of each of the main processes that drive the diurnal $\mathrm{O}_{3}$ budget (deposition, entrainment, chemistry, large (meso) scale 
processes). By using the CHATS observations, we reproduce the boundary layer development during daytime condition for one representative day (Shapkalijevski et al. 2017) and attempt to reconstruct the chemical production and loss using a relatively simple chemistry scheme.

This paper is structured as follows: section 4.2 describes the CHATS site, data treatment, and the methods and formulas to infer fluxes from observed mean gradients. Section 4.3 describes the mixed-layer model, the case study and the budget equations of the boundary-layer state variables and reactants (e.g. $\mathrm{O}_{3}$ ) within the mixed-layer model. In section 4.4, we present and discuss the general characteristics of the CHATS campaign, with emphasis on the differences in the chemistry pre- and post-irrigation. Section 4.4 also includes the results of the gas flux calculations. Next, we present the $\mathrm{O}_{3}$ budget for our case study in section 4.5 and discuss the contribution of deposition, entrainment and chemistry. In section 4.6 , we conclude the paper by summarizing the main findings.

\subsection{Methods}

\subsubsection{Observations and data treatment}

The CHATS experimental site was a mature walnut (Juglans regia) orchard near Dixon, California. The trees were located roughly $7 \mathrm{~m}$ apart with an average height of $10 \mathrm{~m}$ (Patton et al. 2011). The cumulative leaf area index (LAI) was continuously measured throughout the campaign and ranged from 0.7 prior to leaf-out to 2.5 following leaf-out $\left(\mathrm{m}^{2}\right.$ plant area per $\mathrm{m}^{2}$ ground area).

The campaign took place in spring 2007 over three 4-week phases (Patton et al. 2011). This study will focus on the 3rd phase, which follows leaf-out, and during which chemistry observations were taken. During this 3rd phase, the orchard was irrigated in a rotating fashion, with a complete irrigation cycle taking six days (Patton et al. 2011). The observations can then be grouped into pre irrigation (13 to 21 May) and post-irrigation (26 May to 5 June).

The instrumentation was distributed between two main locations: a 30 m vertical tower and a horizontal array of five $12 \mathrm{~m}$ towers (Patton et al. 2011). This study will focus on observations from the $30 \mathrm{~m}$ vertical tower, which was located near the northern boundary of the orchard. This tower had 13 measurement levels for observing micrometeorological and turbulence quantities; six above the canopy, six below the canopy top and one at the canopy top (at $10 \mathrm{~m}$ from the ground surface). At these 13 primary measurement levels wind components and temperature were observed at $60 \mathrm{~s}$ intervals and relative humidity at $2 \mathrm{~s}$ intervals (Patton et al. 2011). Mean vertical profiles of different gases were observed at six heights $(1.5 \mathrm{~m}, 4.5 \mathrm{~m}, 9 \mathrm{~m}, 11 \mathrm{~m}, 14 \mathrm{~m}$ and $23 \mathrm{~m}$ ) at 30 minute intervals. Mixing ratios of $\mathrm{O}_{3}, \mathrm{NO}, \mathrm{NO}_{x}, \mathrm{NO}_{y}, \mathrm{CO}_{2}$ and $\mathrm{H}_{2} \mathrm{O}$ were observed, as well as mixing ratios of five VOCs (Volatile organic compounds). The VOCs were Acetone, Benzene, Toluene, Monoterpenes and Methyl Salicylate (MeSA) (Karl et al. 2008).

In addition to observations from the $30 \mathrm{~m}$ tower, radiation and soil observations were also used. Above canopy broad spectrum upwelling and downwelling radiation was observed at 16 
$\mathrm{m}$, while sub-canopy radiation forcing was observed at $2 \mathrm{~m}$. Soil properties such as soil moisture, heat capacity, and heat flux were collected at $0.05 \mathrm{~m}$ depth. Detailed information about the campaign setup, site description and instruments used can be found in Patton et al. (2011).

30 minute averages of the mixing ratios were used to create daytime diurnal averages of each species, for both the pre and post irrigation period. These diurnal patterns were averaged between 10:00 to 17:00 LT. Micrometeorology observations were used as $30 \mathrm{~min}$. averages.

\subsubsection{Inferring gas fluxes}

In general, $K$-theory is used to calculate fluxes of compounds when fast (eddy covariance) measurements are not available (e.g. Cellier and Brunet 1992). It is a parameterization, which relates the vertical flux of a compound, $F_{c} \equiv \overline{w^{\prime} C^{\prime}}$, to its mean profile, $C(z)$, via the turbulent transfer coefficient for scalars, $K_{C}$ :

$$
\overline{w^{\prime} C^{\prime}}=-K_{C}(z) \frac{\partial \bar{C}(z)}{\partial z} .
$$

Since no vertical fluxes of reactive gases were observed during CHATS, they were calculated from the observed vertical mixing ratio profiles within and above the canopy by using and comparing different adoptions of the flux-gradient method. In this study, transfer coefficients for the vertical fluxes of $\mathrm{O}_{3}$, NO, and $\mathrm{NO}_{2}$ were calculated using four different methods: LOCAL (using observed heat fluxes and potential temperature gradients to calculate $K_{C}$ in Eq. 4.1, MOST (using MoninObukhov similarity theory (Högström 1988) above the canopy to parameterize $K_{C}$ in Eq. 4.1), MOST+RSL (using roughness sublayer parameterization (Harman and Finnigan 2007; 2008) to calculate $K_{C}$ above the canopy) and ILNF (Inverse Localized Near-Field Lagrangian method within the canopy (Raupach 1989a)). Below these four methods of flux calculation are described in more detail.

\section{LOCAL}

To calculate the vertical flux, the LOCAL method uses Eq. 4.1 together with observations of the vertical flux of the potential temperature, $\overline{w^{\prime} \theta^{\prime}}$, and the potential temperature gradients, $d \bar{\theta} / d z$, to calculate $K_{C}$, assuming that the exchange coefficients of gases (reactive gases in our case) are equal to the exchange coefficients for heat: $K_{C}=K_{H}=-\overline{w^{\prime} C^{\prime}} /[d C / d z]$. To calculate the gradient of scalars used within the LOCAL method, the profile of each scalar was fitted to the curve $y=a+b \ln (z)+c \ln (z)^{2}$, using fitting parameters $a, b$ and $c$. This also allowed the interpolation of mixing ratios and gradients at heights where no observations were taken. 


\section{MOST}

Using the Monin-Obukhov Similarity theory (Monin and Obukhov 1954), $K_{C}$ can be described as:

$$
K_{C}(z)=\frac{\kappa(z-d) u_{*}}{\phi_{H}(\zeta)}
$$

where $\kappa$ is the von Kármán constant, $u_{*}$ is the friction velocity, $\phi_{H}$ is dimensionless universal stability function of heat (Högström 1988), and stability $\zeta=(z-d) / L$, where $L$ is the Obuhkov length (e.g. Stull 2009). The stability function for heat is used as it is assumed that chemical species follow this function; $z$ is the height from the ground surface, and $d$ is the displacement height. The displacement height at the CHATS site following leafout is estimated to be $7.5 \mathrm{~m}$ (Shapkalijevski et al. 2016). The MOST method assumes horizontally homogeneous flow and far-field diffusion (Simpson et al. 1998). When considering Eq. 4.2 and integrating Eq. 4.1 between two heights (Moene and Van Dam 2014), the vertical flux becomes:

$$
F_{C}=\frac{\kappa u_{*}\left[C\left(z_{2}-d\right)-C\left(z_{1}-d\right)\right]}{\ln \left(\frac{z_{2}-d}{z_{1}-d}\right)+\psi_{H}\left(\zeta_{1}\right)-\psi_{H}\left(\zeta_{2}\right)},
$$

where $\psi_{H}$ is the integrated form of $\phi_{H}$. The forms of $\phi_{H}$ and $\psi_{H}$ used in this study are based on Högström (1988) and Paulson (1970).

While over a homogeneous and smooth surface gradients in the surface layer follow MOST and can be described by a universal function of atmospheric stability alone, these relationships break down above rough surfaces such as tall vegetative canopies (Thom et al. 1975; Raupach 1979; Garratt 1980; Chen and Schwerdtfeger 1989; Simpson et al. 1998; Shapkalijevski et al. 2016). This is due to the altered turbulence structure and mixing at the canopy vicinity of the roughness sublayer (RSL) (Raupach et al. 1996; Finnigan et al. 2009). This modification is discussed next.

\section{MOST+RSL}

To correct the standard similarity flux-gradient relationships for the presence of the RSL, we followed the method outlined in Harman and Finnigan (2007: 2008). The RSL corrections are based on the inclusion of additional function, $\hat{\phi}_{H}\left(\zeta, \delta_{\omega}\right)$, to Eq. 4.2 :

$$
K_{C}(z)=\frac{\kappa(z-d) u_{*}}{\phi_{H}(\zeta) \hat{\phi}_{H}\left(\zeta, \delta_{\omega}\right)},
$$

where $\delta_{\omega}$ is the vorticity thickness, a characteristic length scale in the RSL representing the length of the turbulent mixing eddies formed at the canopy-atmosphere interface due to the presence of an inflection point in the mean wind at canopy top (Finnigan et al. 2009). Mathematically, $\delta_{\omega}$ is defined as the ratio between the mean horizontal wind speed and its gradient at the canopy top 
$\left(\delta_{\omega}=\bar{u} /[d \bar{u} / d z]\right)$ (Raupach et al. 1996; Harman and Finnigan 2007), and is dependent on canopy density and atmospheric stability. For sparse canopy under near-neutral conditions, Raupach et al. (1996) found that $\delta_{\omega} \simeq 0.5 h_{c}$. This was corroborated by observations of the wind speed and its gradient during the CHATS experiment following leaf-out for the majority of stability classes (from near unstable to near stable) (see Fig. 5 in Shapkalijevski et al. 2016). By including Eq. 4.4, the integrated version of Eq. 4.1 then becomes:

$$
F_{C}=\frac{\kappa u_{*}\left[C\left(z_{2}-d\right)-C\left(z_{1}-d\right)\right]}{\ln \left(\frac{z_{2}-d}{z_{1}-d}\right)+\psi_{H}\left(\zeta_{1}\right)-\psi_{H}\left(\zeta_{2}\right)-\hat{\psi}_{H}\left(\zeta_{1}, \delta_{\omega}\right)+\hat{\psi}_{H}\left(\zeta_{2}, \delta_{\omega}\right)} .
$$

The function $\hat{\psi}_{H}\left(\zeta, \delta_{\omega}\right)$ is the highly non-liner integral function of $\hat{\phi}_{H}\left(\zeta, \delta_{\omega}\right)$ solved by numerical integration (see Harman and Finnigan 2007; 2008).

\section{Inversed Localized Near-Field (ILNF) method}

Within the canopy, it is well known that the turbulent transport of scalar quantities (e.g. heat, water, $\mathrm{CO}_{2}$, pollutant gases) within the canopy is a combination of both diffusive (local) and non-diffusive (non-local or remotely driven) processes due to the changes in turbulence structure near and within rough surfaces (Raupach 1989a; Finnigan et al. 2009). To include these effects, Raupach (1989a) proposed the inversed localized near-field (ILNF) theory to combine the influence of near-field and far-field turbulent transport due to both local and remote sources. This method differs from the previous ones in the sense that the flux-gradient methods above assume that turbulent transport is only diffusive (local). In the ILNF method, the source profile, $S(z)$, is related to the observed mixing ratio profiles $C(z)$ via a dispersion matrix $\mathbf{D}_{i j}$ :

$$
C_{i}-C_{r}=\sum_{j=1}^{m} \mathbf{D}_{i j} S_{j} \Delta z j
$$

where $C_{i}$ is the measured mixing ratio of a scalar quantity within and above the canopy at certain level $i(i=1,2, . ., n)$, and at certain moment in time, relative to the uppermost measured reference mixing ratio, $C_{r}$, above the canopy. The index $j$ refer to levels of the scalar source/sink distribution within the canopy $(j=1,2, . ., m)$ ), which are different than the levels of the concentration profiles (see Fig. 7 in Raupach 1989a). $n$ and $m$ represent the number of mixing ratio and flux levels respectively, with the condition that $m<n$. This dispersion matrix $\mathbf{D}_{i j}$ is a property of the vertical velocity variance, $\sigma_{w}$, and the Lagrangian integral time scale $T_{L}$. Both $\sigma_{w}$ and $T_{L}$ are parameterized by Raupach (1989a) using empirical functions. For the purpose of this study, we adjusted the coefficients of these functions by using the CHATS observed profiles of $\sigma_{w}$ and $T_{L}$. 
By solving Eq. 4.6 for $S_{i}$, the flux of a scalar can then be found via:

$$
F(z)=\int_{0}^{z} S(z) d z
$$

An important improvement of this method is that it can account for counter-gradient fluxes (Denmead and Bradley 1987; Raupach 1989a). Detailed information about the ILNF theory can be found in Raupach (1989a). The ILNF method of flux calculation was used to calculate fluxes at 2, 4, 6 and $8 \mathrm{~m}$ within the canopy.

The LOCAL method was used to calculate the gas fluxes within and above the canopy. The MOST and MOST+RSL were used to calculate the gas fluxes only above the canopy, while the ILNF was used only within the canopy. The fluxes of $\mathrm{O}_{3}, \mathrm{NO}$ and $\mathrm{NO}_{2}$ were inferred from the mixing ratio observations averaged between 10:00 to 17:00 LT for pre- and post-irrigation periods, as well as on 27 May 2007 (147th Julian day of 2007), our case study day. Only fluxes on 27 May 2007 were calculated using the ILNF method were inferred from the mixing ratio observations averaged between 10:00 to 17:00 LT.

\subsection{Modelling the ozone budget}

\subsubsection{Model and case study}

In this study the temporal evolution of $\mathrm{O}_{3}$ budget for a representative day at CHATS was calculated by combining modelling and observations. The atmospheric model used here was the zerodimensional mixed-layer model (Lilly 1968; Betts 1973; Tennekes and Driedonks 1981). The mixed-layer model was coupled to a surface soil-vegetation scheme to account for the surface momentum and energy forcing in the CBL system (van Heerwaarden et al. 2009). In addition, a relatively simple chemical scheme was introduced to the mixed-layer model to simulate chemistry within the boundary layer (Vilà-Guerau de Arellano et al. 2009; Ouwersloot et al. 2012; van Stratum et al. 2012). The model is called MiXed-Layer CHemistry model (MXLCH). Recently, Shapkalijevski et al. (2017) introduced the RSL effects in the surface scheme of the MXLCH model to study the effects of relatively tall and sparse orchard canopy on the CBL dynamics during CHATS. Here, by extending the case study on the mixed-layer thermo(dynamics), we use the MXLCH-model to study the combined effects of deposition, entrainment and chemistry on the ozone budget over the CHATS canopy.

The MXLCH-model solves the temporal evolution of the mixed-layer variables of the thermodynamic and chemical species together with an equation for the boundary-layer height and the jump of the mixed-layer quantity in the free troposphere (see Eq. 4.10). The governing prognostic 
equation of the mixed-layer ozone reads:

$$
\frac{\partial\left\langle C_{O_{3}}\right\rangle}{\partial t}=\frac{\left(\overline{w^{\prime} C_{O_{3}}^{\prime}}\right)_{s}-\left(\overline{w^{\prime} C_{O_{3}}^{\prime}}\right)_{e}}{h}+\left\langle S_{O_{3}}\right\rangle-\left(u \frac{\partial\left\langle C_{O_{3}}\right\rangle}{\partial x}+v \frac{\partial\left\langle C_{O_{3}}\right\rangle}{\partial y}\right)
$$

and describes how the mixing ratio of $\mathrm{O}_{3}$ changes over time in the well-mixed boundary layer (e.g. Vilà-Guerau de Arellano et al. 2015). The first two terms on the right hand side of the "=" are the surface flux and the entrainment ozone flux into the boundary layer at height $h$. The third term is the chemical production or loss of $\mathrm{O}_{3}$ due to chemical reactions, while the last term represents horizontal advection of $\mathrm{O}_{3}$ in the boundary layer.

The mixed-layer assumptions only hold during diurnal convective conditions in which the conserved boundary-layer state variables (Lilly 1968; Tennekes and Driedonks 1981) and the reactants (Ouwersloot et al. 2012) are characterized by vertical profiles that are constant with height. Therefore, we focus here on the $\mathrm{O}_{3}$ daytime variability. This case-study day (147 DOY) was chosen based on the dominant winds from the south, meaning best fetch over the orchard canopy, leading to a well-developed RSL (see Chapter 3). To complete the $\mathrm{O}_{3}$ budget on Day 147, simultaneously the (thermos)dynamics of mixed-layer potential temperature, specific humidity, wind speed and the boundary layer height were simulated (see Chapter 3 for details). Since the $\mathrm{h}$ included in Eq. 4.8 was initialized and well represented by the mixed-layer model for our case study in Chapter 3, we focus here on explaining the other terms in the ozone budget related to the boundary conditions (surface and entrainment fluxes), chemistry, and the advection. In the following sections $(4.3 .2-4.3 .5)$ each of the processes represented in Eq. 4.8 is explained, including a description how they are incorporated in the MXLCH model.

\subsubsection{Entrainment}

The entrainment of $\mathrm{O}_{3}$ from the free troposphere into the boundary layer is represented through the term $\left(\overline{w^{\prime} C_{O_{3}}^{\prime}}\right)_{e}$. Ozone entrainment in the MXLCH model is modelled as a product between the entrainment velocity, $w_{e}$, and the difference in the mixing ratio of $\mathrm{O}_{3}$ in the free troposphere and in the mixed-layer $\left(\Delta C_{O_{3}}=C_{O_{3_{F T}}}-\left\langle C_{O_{3}}\right\rangle\right)$ with a negative sign (e.g. Vilà-Guerau de Arellano et al. 2015):

$$
\left.\overline{\left(w^{\prime} C_{O_{3}}^{\prime}\right.}\right)_{e}=-w_{e} \Delta C_{O_{3}} .
$$

$w_{e}$ is linked to the growth in boundary-layer height (Vilà-Guerau de Arellano et al. 2015). At the top of the mixed layer,

$$
\frac{\partial \Delta C_{O_{3}}}{\partial t}=\frac{\partial C_{O_{3_{F T}}}}{\partial t}-\frac{\partial\left\langle C_{O_{3}}\right\rangle}{\partial t}=\gamma_{O_{3}}\left(\frac{d h}{d t}-w_{s}\right)-\frac{\partial\left\langle C_{O_{3}}\right\rangle}{\partial t},
$$

where $w_{s}$ represents the subsidence velocity (see Chapter 3 ) and $\gamma_{O_{3}}$ is the free tropospheric lapse rate for $\mathrm{O}_{3}$. Note that similar equations as Eqs. $4.8-4.10$ hold for the mixed-layer thermos- 
dynamical variables (potential temperature, specific humidity, wind velocity) and other 'passive' (e.g. carbon dioxide) or 'active' (e.g. $\mathrm{NO}, \mathrm{NO}_{2}$, VOCs) atmospheric constituents(Vilà-Guerau de Arellano et al. 2015).

\subsubsection{Dry deposition}

The second term in Eq. 4.8, $\left(\overline{w^{\prime} C_{O_{3}}^{\prime}}\right)_{s}$, defines the lower boundary in the MXLCH, and represents the surface exchange of ozone. Dry deposition refers to loss of $\mathrm{O}_{3}$ through physical contacts with surfaces. A major pathway of $\mathrm{O}_{3}$ deposition is deposition through the stomata, with other pathways being deposition onto surfaces such as leaf cuticles or the soil (Fares et al. 2012). In the MXLCH, and from theoretical perspective, the relation between the effective surface fluxes of $\mathrm{O}_{3}, F_{O_{3}}$ in the surface layer (assumed constant in height) and the ozone (reactants) mixing ratio, $\mathrm{C}_{O_{3}}$ at specific reference height $z_{r}$ is:

$$
F_{O_{3}}=\left(\overline{w^{\prime} C_{O_{3}}^{\prime}}\right)_{s}=v_{d}\left(z_{r}\right) C_{O_{3}}\left(z_{r}\right)
$$

Large-scale atmospheric models use a deposition velocity in combination with the concentration in the lowest grid box (typically with a height of $25 \mathrm{~m}$ ) to calculate the deposition flux of ozone. As such, $v_{d}$ represents the combined effects of aerodynamic resistance, diffusion, and uptake by soil and/or vegetation (Wesely and Hicks 1977). In our application, we evaluate the heightdependent deposition velocity using Eq. 4.11. The measured profiles of the ozone mixing ratios and the derived ozone flux profiles can be used to derive a height-dependent $v_{d}$ profile. This profile depends on the way fluxes are derived from the mixing ratios and thus differs for LOCAL, MOST, and MOST+RSL. To study the potential effects of the RSL parameterization on vd and hence on calculated deposition fluxes in large scale models, we evaluate $v_{d}$ at $2.5 \mathrm{~m}$ above the canopy and apply this value in the MXLCH model. The height of $2.5 \mathrm{~m}$ above the canopy is a typical altitude for conducting measurements and is the typical altitude of the center of a grid box in large-scale models (12.5 $\mathrm{m}$ above the surface).

Appendix 4B presents an example for the derived ozone deposition velocity. Using the available observations, we use diurnally averaged $v_{d}$ values evaluated at $2.5 \mathrm{~m}$ above the canopy for $\mathrm{O}_{3}, \mathrm{NO}$, and $\mathrm{NO}_{2}$.

\subsubsection{Chemistry}

Chemical production and loss within the boundary layer is represented in Eq. 4.8 by $S_{O_{3}}$. The chemistry scheme in MXLCH that we use in our experiment has moderate complexity and has been shown to capture well the majority of the relevant chemical pathways in the formation of ozone in rural and semi-rural regions (e.g. Ouwersloot et al. 2012; van Stratum et al. 2012). A complete list of the reactions used in the MXLCH-model can be found in Table 4.1. In the following, we will define some useful concepts that can help our analysis. 
Table 4.1: Chemical reaction scheme used in the numerical experiments with $T$ being the absolute temperature in Kelvins and $\chi$ the solar zenith angle. The reaction rates are taken from the International Union of Pure and Applied Chemistry (IUPAC) (http://iupac.pole.ether.fr) and can be found in Vilà-Guerau de Arellano (2015). All first-order rate constants are in $\left[\mathrm{s}^{-1}\right]$, secondorder in $\left[\mathrm{cm}^{3}\right.$ molecules $\left.{ }^{-1} \mathrm{~s}^{-1}\right]$ and all concentrations are calculated in units molecules $\mathrm{cm}^{-3}$. The species between brackets do not enter explicitly in the calculation of the chemical transformations, as their concentrations are very large compared to chemical production. (hv) denotes a photolytic conversion. 'REST' stays for products that are not further evaluated in the chemical scheme.

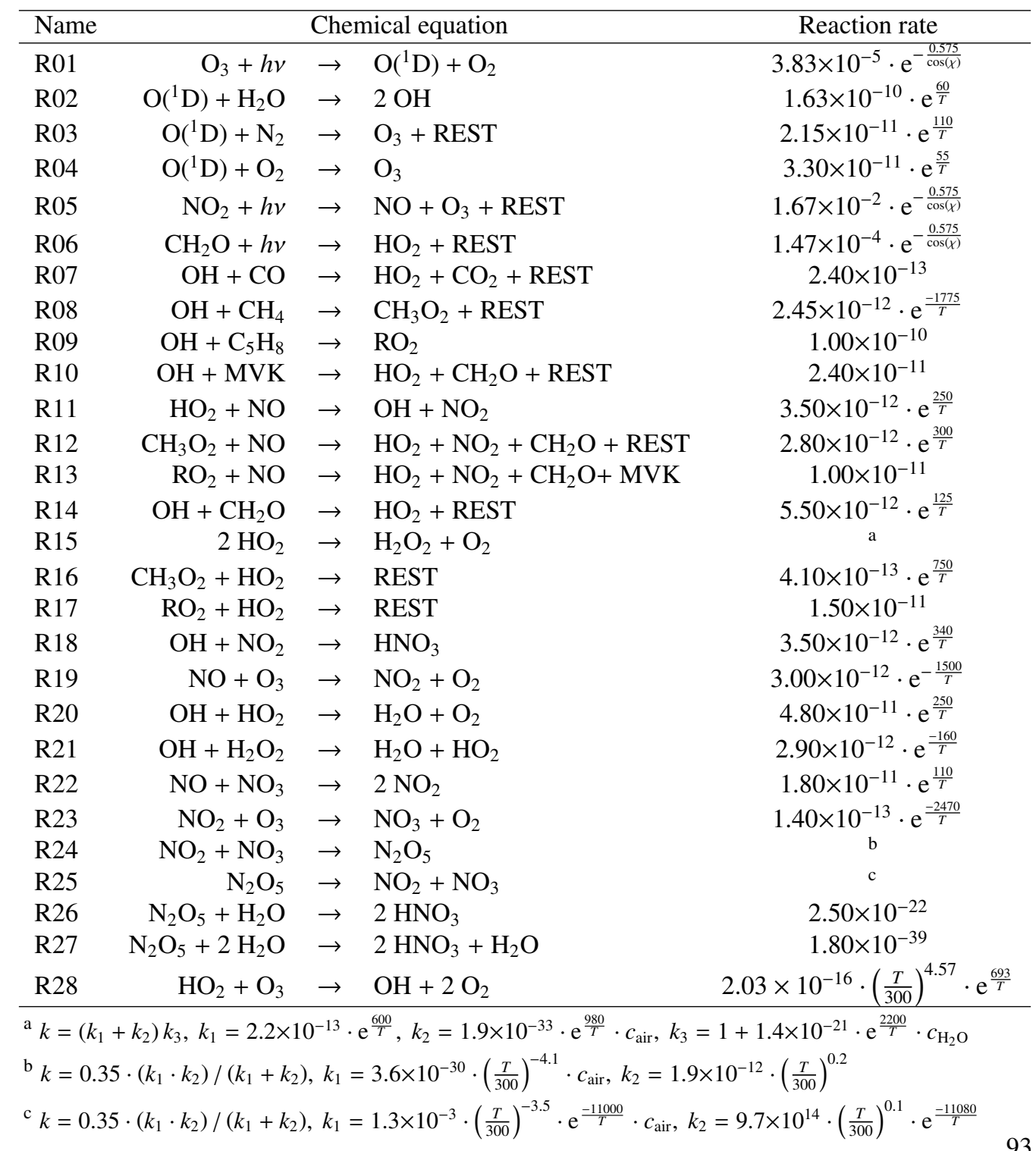


Ozone in the troposphere is primarily formed in the presence of sunlight through photochemical reactions of nitrogen oxides and hydrocarbon (Fares et al. 2012). Dissociation reaction R05 (Table 4.1) and subsequent regeneration of $\mathrm{NO}_{2}$ via reaction with $\mathrm{NO}$ and $\mathrm{O}_{3}$ is sufficiently fast in sunlit conditions and moderate $\mathrm{NO}_{x}\left(\mathrm{NO}_{\mathrm{x}}=\mathrm{NO}+\mathrm{NO}_{2}\right)$ mixing ratios $(<10 \mathrm{ppb})$ that these species are in dynamic equilibrium on a time scale of $\sim 100$ seconds (Carpenter et al. 1998; Mannschreck et al. 2004), which can be described by:

$$
C_{O_{3}}=\frac{j_{N O_{2}} C_{N_{2}}}{k_{19} C_{N O}}
$$

where $j_{\mathrm{NO}_{2}}$ is the photolysis frequency of $\mathrm{NO}_{2}(\mathrm{R} 05)$, and $k_{19}$ is the temperature dependent reaction rate for R19 (Griffin et al. 2007). This is referred to as the photostationary state (PSS). The PSS parameter, or Leighton ratio $(\varphi)$, is defined as:

$$
\varphi=\frac{j_{N_{2}} C_{N O_{2}}}{k_{19} C_{N O} C_{O_{3}}}
$$

This photostationary state mainly depends on the photolysis of $\mathrm{NO}_{2}$, and when other reactions converting $\mathrm{NO}$ to $\mathrm{NO}_{2}(\mathrm{R} 11, \mathrm{R} 12, \mathrm{R} 13)$ and local emissions of these species are negligible, the Leighton ratio is expected to be equal to unity (Mannschreck et al. 2004). A Leighton ratio near unity is mainly observed in polluted areas (Carpenter et al. 1998; Thornton et al. 2002; Yang et al. 2004), while in rural or isolated areas with high sunlight Leighton ratios of up to 3 have been observed (e.g. Volz-Thomas et al. 2003). The value of $\varphi$ deviates positively from unity when pathways, other than $\mathrm{R} 19$, convert $\mathrm{NO}$ to $\mathrm{NO}_{2}$ (Griffin et al. 2007). In conditions with low $\mathrm{NO}_{\mathrm{x}}$ levels, the reactions between $\mathrm{NO}$ and peroxy radicals $\mathrm{HO}_{2}, \mathrm{RO}_{2}$ and $\mathrm{CH}_{3} \mathrm{O}_{2}$ form the major pathway causing positive deviation from the photostationary state (Carpenter et al. 1998; Mannschreck et al. 2004)(e.g. R11, R12, R13). These reaction pathways additionally lead to the formation of $\mathrm{O}_{3}$ via the subsequent dissociation of $\mathrm{NO}_{2}(\mathrm{R} 05)$. As the reaction rates of R11 and R13 are similar (DeMore et al. 1997), the concentration of peroxy radicals $\mathrm{RO}_{2}$ $\left(\mathrm{HO}_{2}+\mathrm{RO}_{2}+\mathrm{CH}_{3} \mathrm{O}_{2}\right)$ is given by:

$$
C_{P_{2}}=(\varphi-1) \frac{k_{19} C_{O_{3}}}{k_{11}},
$$

where $\mathrm{k}_{11}$ is the reaction rate of $\mathrm{R} 11$.

\subsubsection{Advection}

The final term in Eq. 4.8 is advection of ozone. This refers to the horizontal transport of $\mathrm{O}_{3}$ mixing ratio into or out of the mixed layer at the CHATS site.

The CHATS site is located within the Sacramento Valley and as a result is subject to com- 
plex flow patterns. The Sacramento Valley has complex topography, being bordered by the Coast Ranges and the Sierra Nevada (Bianco et al. 2011). Differential heating due to altitude produces local, thermally-driven diurnal flows within the Sacramento valley (Bao et al. 2008). In addition, differential ocean-land heating together with weak synoptic flows leads to marine air being transported from the Sacramento Bay area through the Sacramento valley (Bao et al. 2008; Bianco et al. 2011). Important for our case study is the advective transport of cold and humid air from the San Francisco Bay area during the day, which affects the boundary-layer (thermo)dynamics (Chapter 3).

The proposed effects of these regular diurnal flows on pollution transport within the Sacramento Valley are complex and often contrary. The influx of marine air with the up-valley flow can ventilate existing pollution within the valley (i.e. advective outflow of pollution). However, pollution from the heavily populated San Francisco area can also be transported into the valley (Bao et al. 2008). Thermally driven upslope flows during the day induces subsidence, suppressing the development of the boundary layer and confining pollution to a thinner boundary layer (as observed during majority of the CHATS days, as well during our case study day), thus raising mixing ratios (Bao et al. 2008). These same upslope flows transport have also been proposed to transport polluted air up the Sierra Nevada foothills, cleaning the valley (Dillon et al. 2002). Bao et al. (2008) suggested that night-time, downslope winds can circulate pollutants back through the valley. Dillon et al. (2002) however, proposed that katabatic flows introduces clean, regional air into the Sacramento Air Basin, lowering pollutant levels. Due to the relatively low ozone and high $\mathrm{NO}_{\mathrm{x}}$ mixing ratios over the CHATS site (especially during the case study day), a negative advection of ozone and positive of $\mathrm{NO}_{\mathrm{x}}$ (last term in Eq. 4.8) will be taken into account in our study. The negative advection of ozone over the CHATS site would correspond to a situation with low ozone mixing ratios over the San Francisco Bay area (due to ozone titration by abundant NO via $\mathrm{O} 3$ + NO (R14) (Fujita et al. 2001; Fujita et al. 2005), advecting ozone-poor and $\mathrm{NO}_{\mathrm{x}}$-rich air masses. Additionally, positive advection of $\mathrm{NO}_{\mathrm{x}}$ during CHATS may be due to the highway located south of the orchard. This $\mathrm{NO}_{\mathrm{x}}$ then influences the ozone production regime over the CHATS. It is worth mentioning here the nonlinearity of ozone formation for a given variety of $\mathrm{NO}_{\mathrm{x}}$ conditions (e.g. Jacob 1999). This moderate-to-high $\mathrm{NO}_{\mathrm{x}}$ levels may either lead to ozone production (via VOCs $->\mathrm{RO}_{2}+\mathrm{NO}->\mathrm{NO}_{2}->\mathrm{O}_{3}$ or to ozone destruction (i.e. $\mathrm{NO}+\mathrm{O}_{3}->\mathrm{NO}_{2}$ $\left.(+\mathrm{OH})->\mathrm{HNO}_{3}\right)($ Rohrer and Berresheim 2006).

\subsection{Atmospheric chemistry during CHATS}

\subsubsection{General characterization}

We start our analysis with the general meteorological and chemical characteristics of the experimental site. The CHATS site shows meteorological conditions typical of a Mediterranean climate, with the average temperature during the campaign peaking at $27^{\circ} \mathrm{C}$ by $15: 00 \mathrm{LT}$ pre irrigation 

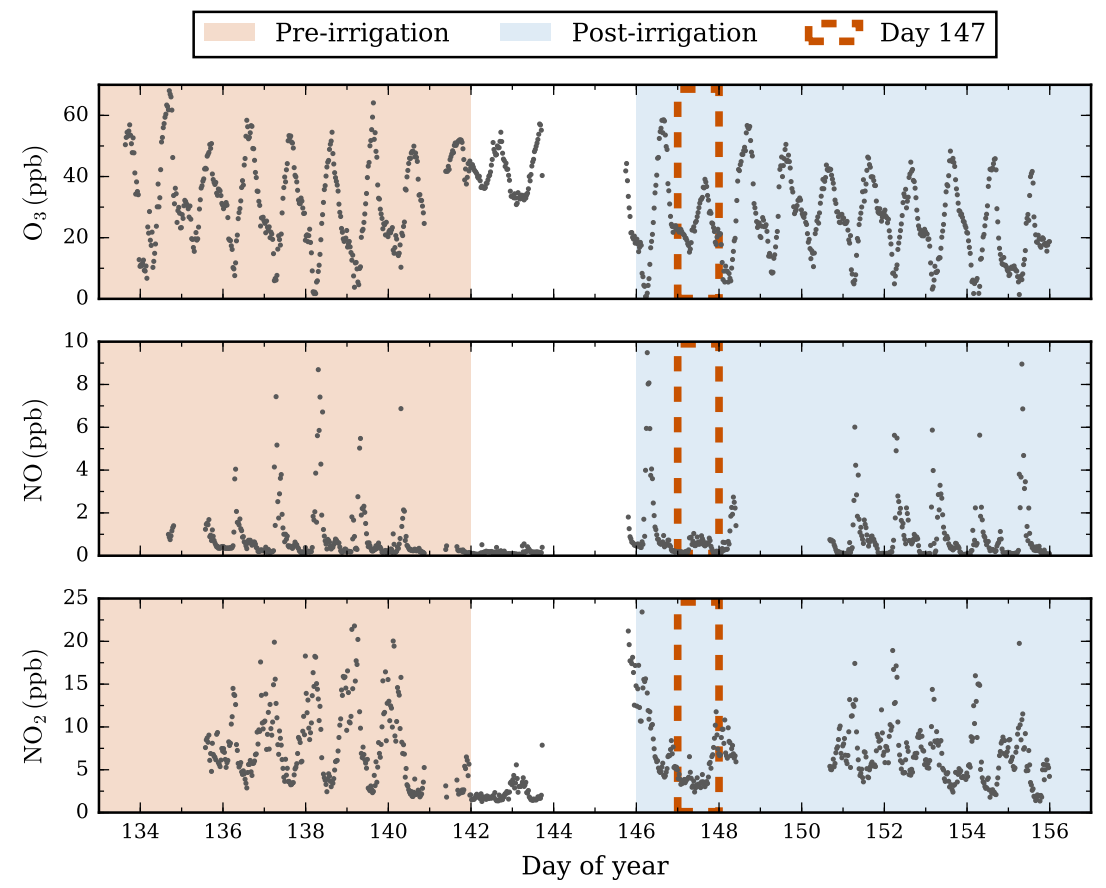

Figure 4.1: Time series of $\mathrm{O}_{3}, \mathrm{NO}$ and $\mathrm{NO}_{2}$ concentrations, taken at $11 \mathrm{~m}$ above the ground surface ( $1 \mathrm{~m}$ above the canopy top). The orange shaded area indicates the pre-irrigation period, while the blue shaded area shows the post-irrigation period. The dashed line boxes indicate the case study day (27 May 2007 or 147 DOY).

( $26^{\circ} \mathrm{C}$ post irrigation), and the specific humidity reaching a peak of $7.5 \mathrm{~g} \mathrm{~kg}^{-1}$ pre irrigation (and $8.5 \mathrm{~g} \mathrm{~kg}^{-1}$ post irrigation). Winds were generally from the south and, as typical in the central Californian region, the boundary layer was shallow at around $700 \mathrm{~m}$ (Bianco et al. 2011). During CHATS, the boundary layer height was deeper during the pre-irrigation period compared to the post-irrigation period (1200 $\mathrm{m}$ and $700 \mathrm{~m}$, respectively).

Figure 4.1 shows the observed mixing ratios of 3 chemical compounds observed during the CHATS campaign: $\mathrm{O}_{3}$, $\mathrm{NO}$ and $\mathrm{NO}_{2}$. Figure 4.1 also shows the timing of the pre- and postirrigation periods and the case study day. The diurnal pattern of $\mathrm{O}_{3}, \mathrm{NO}$ and $\mathrm{NO}_{2}$ is clearly visible, with mixing ratios being comparable pre and post irrigation. $\mathrm{NO}_{x}$ mixing ratios are generally higher at night and in the early morning due to accumulation of fresh emissions in the shallow night time boundary layer. Ozone mixing ratios peak in the afternoon, following daytime ozone production and possibly entrainment from the overlying free atmosphere. At night, $\mathrm{O}_{3}$ mixing ratios are much lower, due to deposition and titration by NO (i.e. reaction R19, producing 

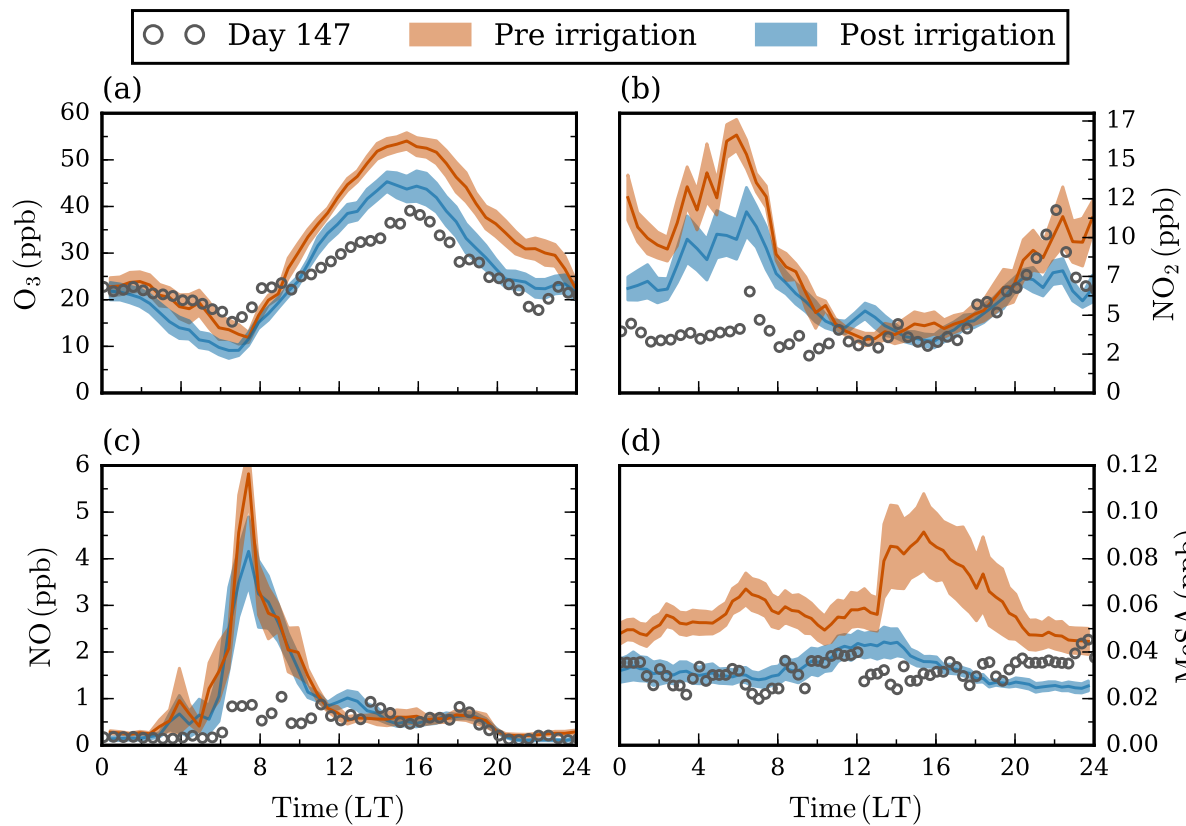

(d)

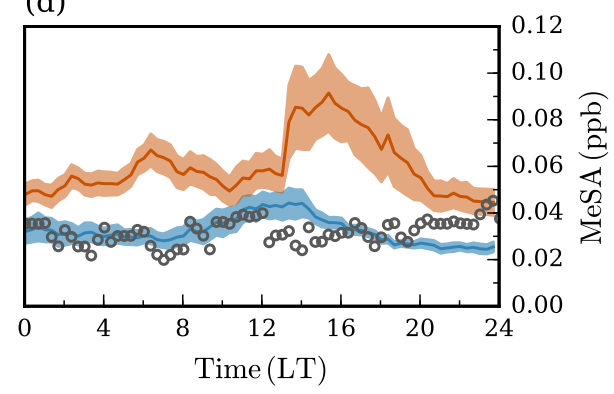

Figure 4.2: Diurnal evolution of (a) $\mathrm{O}_{3},(\boldsymbol{b}) \mathrm{NO}_{2}$, (c) $\mathrm{NO}$ and (d) MeSA (see text for full name) mixing ratios averaged above the canopy $(11,14$ and $23 \mathrm{~m}$ ) for Day 147 and for the pre-and post- irrigation periods. The shaded area represents the standard error of the mean.

$\mathrm{NO}_{2}$ ). These observations support the general diurnal behaviour of the boundary layer: convective during daytime, and stable during the night. Other observed chemical species, not explicitly treated in this study, were $\mathrm{CO}_{2}$ and additional VOCs. $\mathrm{CO}_{2}$ was around $390 \mathrm{ppm}$ (daily average) with no noticeable difference pre and post irrigation (not shown). All observed VOC mixing ratios were consistently higher pre irrigation compared to post-irrigation mixing ratios. Acetone peaked at $5 \mathrm{ppb}$, with monoterpenes reaching $0.25 \mathrm{ppb}$ pre irrigation. Benzene and toluene showed little diurnal variation and remained below $0.4 \mathrm{ppb}$. The average mixing ratios of MeSA, a VOC compound emitted by plants under drought stress (Karl et al. 2008), are shown in Fig. 4.2d and are discussed later on.

Figure 4.2 shows the diurnal averages of the mixing ratios presented in Fig. 4.2 and for MeSA, averaged over the pre and post-irrigation period, and for the case study day 147 . The $\mathrm{O}_{3}$ diurnal profiles observed at CHATS are comparable to other rural regions in Central California during spring, with the average peak mixing ratio reaching between 45 to $55 \mathrm{ppb}$. Fares et al. (2012) found $\mathrm{O}_{3}$ mixing ratio above a Californian orange orchard to be around $55 \mathrm{ppb}$ in spring 2009, with daytime peaks in the summer exceeding $100 \mathrm{ppb}$. During summer months $\mathrm{O}_{3}$ mixing ratio in the Central California regions often exceed the federal threshold (8-hour average exceeding 85 
ppb) (Murphy et al. 2007). The morning peak of $\mathrm{NO}_{2}$ reached up to $17 \mathrm{ppb}$ before dropping to $3 \mathrm{ppb}$ at midday, while the morning peak of NO reached up to $6 \mathrm{ppb}$, with mixing ratios during midday of around $0.5 \mathrm{ppb}$ (Fig. 4.2). At sunrise, $\mathrm{NO}_{2}$ is photolysised and starts cycling between $\mathrm{NO}$ and $\mathrm{NO}_{2}$ (see also Seok et al. 2013). This leads to the morning build-up of NO, before the boundary layer begins to grow and to dilute the $\mathrm{NO}_{x}$ mixing ratios. The NOx mixing ratios during CHATS are similar to findings by Murphy et al. (2007) who found Californian daytime mean mixing ratios of $\mathrm{NO}_{x}$ to be between 5 and $18 \mathrm{ppb}$. These high levels of $\mathrm{NO}_{x}$ are mostly due to traffic emissions (Murphy et al. 2007). Min et al. (2014) investigated fluxes of $\mathrm{NO}_{x}$ within the canopy of a pine forest on the Sierra Nevada mountain slopes. They found lower mixing ratios of $\mathrm{NO}$ and $\mathrm{NO}_{2}$ than observed at the CHATS site, due to an increased distance from urban areas compared to the CHATS site. NO mixing ratios above the canopy ranged from 10 to $100 \mathrm{ppt}$ while the mixing ratio of $\mathrm{NO}_{2}$ varied from 80 to $550 \mathrm{ppt}$ in the study by Min et al. (2014). It thus appears that advection of $\mathrm{NO}_{x}$ dominates over possible emissions from managed soils.

Significant mixing ratios of MeSa were observed (0.1 ppb) during CHATS, although typical ecosystem mixing ratios of MeSA remain uncertain (Karl et al. 2008). MeSA is a biologically active compound which is thought to be synthesized by plants as a response to temperature stress (Karl et al. 2008). The average MeSa mixing ratios are consistently higher pre irrigation compared to post irrigation, likely caused by drought stress which increases daytime leaf temperatures and therefore MeSA emission. In addition to MeSA levels being higher pre irrigation, $\mathrm{O}_{3}$ mixing ratios were around $5 \mathrm{ppb}$ higher prior to irrigation. The nighttime build-up in $\mathrm{NO}_{2}$ was also around $3 \mathrm{ppb}$ higher before irrigation. This difference pre and post irrigation is seen again in the vertical profile of these species (Fig. 4.3) and is discussed further in Sect. 4.4.3.

Another interesting characteristic in the diurnal trend of NO and NO2 mixing ratios is the much smaller early morning peak for the case study day (Fig. 4.2b and 4.3c). As the case study day is a Sunday, this lack of the morning peak is likely due to the 'weekend effect'. Day of the week differences in the chemical composition of the boundary layer are often observed, and the Sacramento valley has been the focus of many studies examining this 'weekend effect' on $\mathrm{O}_{3}$ concentrations (Altshuler et al. 1995; Murphy et al. 2007). This weekend effect is hypothesized to be due to the differentiated anthropogenic emissions of $\mathrm{O}_{3}$ precursors on weekdays and weekends, in particular NOx emissions from traffic. Reduced emission of $\mathrm{NO}_{x}$ in the weekend means that rural areas are likely to see lower $\mathrm{O}_{3}$ mixing ratios on weekends, as seen in Fig. 4.2a. As there is a major road to the south of the orchard, this weekend effect due to traffic emissions is expected to strongly influence chemistry of the CHATS site - particularly with southerly winds as on Day 147. As MeSA is biologically emitted and the mixing of MeSA on Day 147 shows no significant difference from the post irrigation mean mixing ratio, this is further evidence of the weekend effect on anthropogenic $\mathrm{NO}_{x}$ emissions.

Figure 4.3 shows the vertical profile of the mixing ratios averaged from 10:00 to 17:00 LT (i.e. during well-mixed conditions), for each irrigation period and for the case study day. Here 


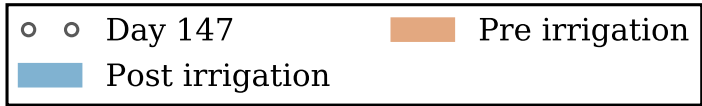

(a)

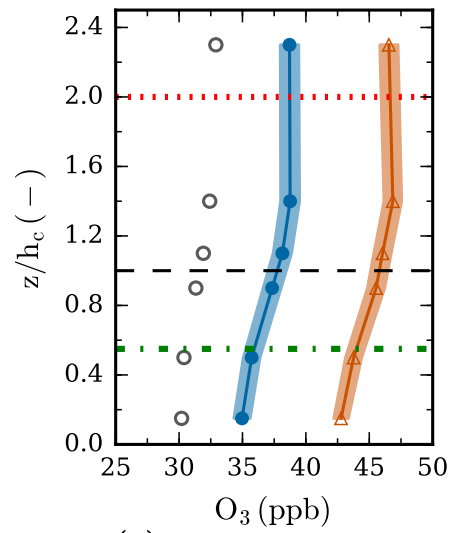

(c)
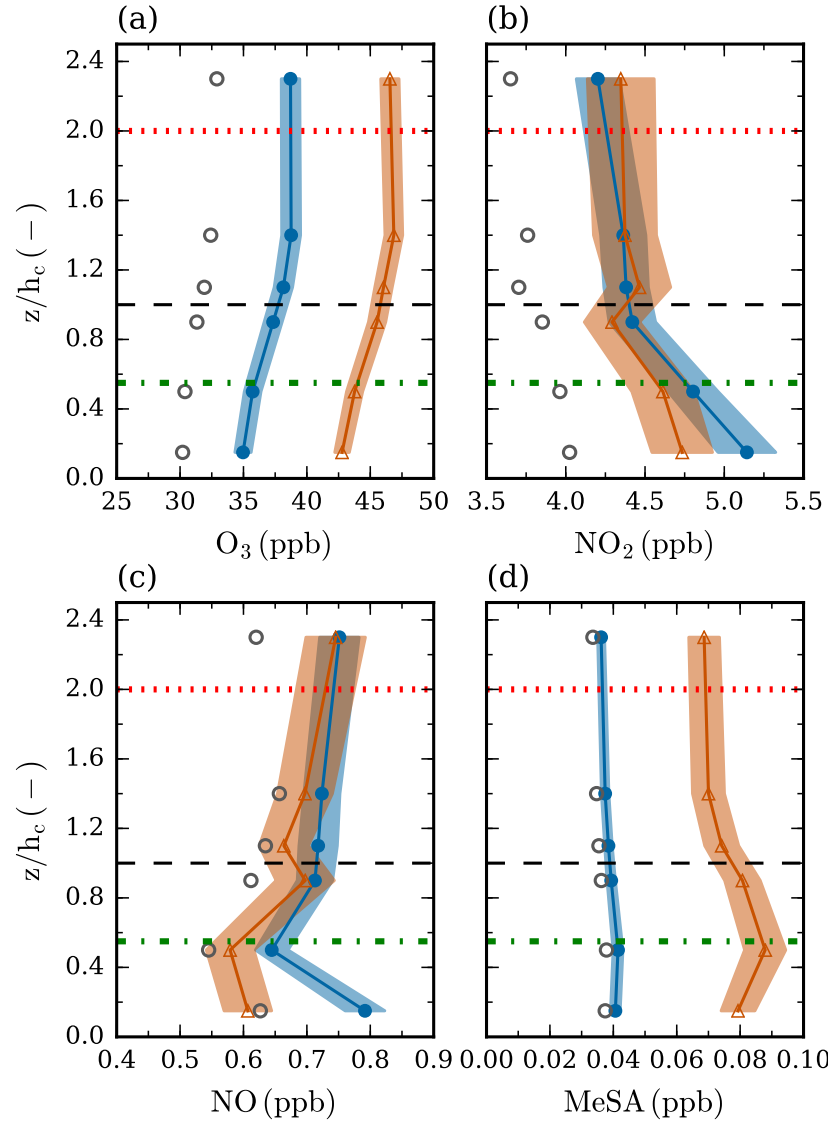

(d)

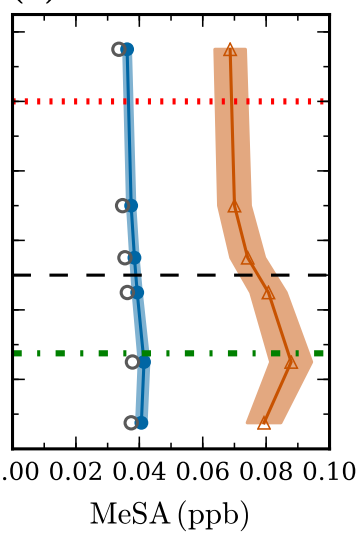

Figure 4.3: Vertical profiles of (a) $\mathrm{O}_{3}$, (b) $\mathrm{NO}_{2}$, (c) $\mathrm{NO}$ and (d) MeSA mixing ratios averaged between 10:00 and 17:00 LT on Day 147, and for the pre- and post-irrigation periods. The shaded area represents the standard error of the mean. The red dashed line indicates the height of the RSL, while the black dashed line and the green dashed-dotted line mark the canopy height and the bottom of the crown canopy, respectively. 
positive gradients indicate a negative (downward) flux. In general, the vertical gradients of all species are small, corresponding to a well-mixed roughness sub-layer and relatively small fluxes. This indicates that vertical mixing is maintained throughout the canopy, potentially due to its sparsity. The daytime $\mathrm{O}_{3}$ mixing ratio shows a small positive vertical profile indicative of ozone deposition, with mixing ratios increasing by 5 ppb over $21.5 \mathrm{~m}$. As shown in Fig. 4.3, the $\mathrm{O}_{3}$ mixing ratio decreases after irrigation on average by about $7 \mathrm{ppb}$. The gradient of NO is negative inside the canopy, while the gradient of $\mathrm{NO}_{2}$ is positive above the canopy. Both $\mathrm{NO}$ and $\mathrm{NO}_{2}$ show a disruption of this gradient at the top of the crown. This may indicate (i) the influence of a strong local source/sink for these species near the canopy top or within the canopy or (ii) horizontal transport from pollutants above the canopy. Below the canopy NO mixing ratios show a strong divergence pre and post irrigation. This may be due to increased emission of NO from the soil following increased soil moisture and is discussed further in the next section. It is also interesting to notice the higher MaSA mixing ratio pre irrigation, indicating increased (VOCs) emission, especially at canopy level.

\subsubsection{Inferring fluxes of radicals from observed mean mixing ratios}

In appendix 4A we show that the use of the flux-gradient methods MOST and MOST+RSL is generally valid above the canopy. The same holds for the ILNF method within the canopy to calculate fluxes from observed mean wind speed and potential temperature profiles. Important for our study is the improved calculation of scalar fluxes ( $\mathrm{H}$ in this case) when using MOST+RSL compared with MOST, since this method takes the RSL effects in the flux parameterization into account. In what follows, we apply these methods to infer the gas fluxes from their mixing ratio profiles.

Figure 4.4 shows the fluxes of $\mathrm{O}_{3}, \mathrm{NO}$ and $\mathrm{NO}_{2}$ on Day 147 calculated by all four methods, in and above the canopy. There is a relatively good correspondence between the methods, with the profile shapes of the fluxes being consistent across all methods. The largest fluxes are seen at the top of the canopy. $\mathrm{O} 3$ shows strong negative fluxes within and just above the canopy, indicating deposition, between -0.9 to $-0.5 \mathrm{ppb} \mathrm{m} \mathrm{s}^{-1}$. These fluxes are comparable to fluxes reported above other canopies. Fares et al. (2012) found spring $\mathrm{O}_{3}$ fluxes of $-0.94 \mathrm{ppb} \mathrm{m} \mathrm{s}^{-1}$ above an orange orchard in Central California. Above a pine forest in the Sierra Nevada Mountains fluxes of -1.04 ppb $\mathrm{m} \mathrm{s}^{-1}$ were recorded (Fares et al. 2010). Other observations of ozone fluxes above a range of canopy types show values around $-0.78 \mathrm{ppb} \mathrm{m} \mathrm{s}^{-1}$ (Mikkelsen et al. 2000; Fares et al. 2013; Fares et al. 2014). Above the RSL layer, we calculate positive NOx fluxes with values of 0.02 ppb m s${ }^{-1}$ and $0.04 \mathrm{ppb} \mathrm{m} \mathrm{s}^{-1}$ for $\mathrm{NO}$ and $\mathrm{NO}_{2}$, respectively. This is comparable to Farmer and Cohen (2008) who found summer-time noon fluxes of $\mathrm{NO}$ and $\mathrm{NO}_{2}$ above a pine canopy in the Sierra Nevada Mountains of 0.054 and $0.30 \mathrm{ppb} \mathrm{m} \mathrm{s}^{-1}$ respectively. Min et al. (2014) found NO and NO2 fluxes above the same canopy of 0.32 and $0.67 \mathrm{ppb} \mathrm{m} \mathrm{s}^{-1}$, smaller than calculated at the CHATS site. Unlike in Min et al. (2014), we found negative NO fluxes below the canopy on day 


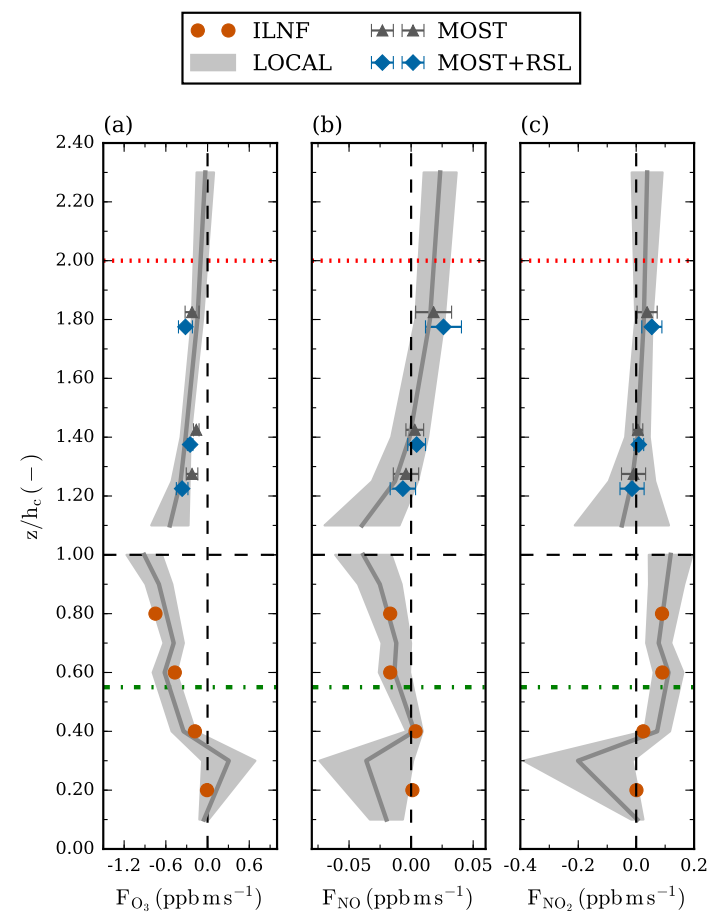

Figure 4.4: Vertical flux profiles of (a) $\mathrm{O}_{3},(\boldsymbol{b}) \mathrm{NO}$ and (c) $\mathrm{NO}_{2}$, calculated via the four different methods, averaged between 10:00 to 17:00 LT on Day 147. See Fig. 4.3 for a description of the shaded area and horizontal lines.

147, whereas they found soil emissions of NO between $0.05-0.08 \mathrm{ppb} \mathrm{m} \mathrm{s}^{-1}$. Furthermore, the observed flux divergence in Fig. 4.4, especially within the canopy can be related to: (i) differential advection (i.e. advection of $\mathrm{NO}_{x}$ in the $\mathrm{BL}$ ) and (2) chemistry. Considering the photostationary state $\mathrm{NO}+\mathrm{O}_{3} \leftrightarrow \mathrm{NO}_{2}$, one would expect that there will be less light in the canopy, shifting the equilibrium in the reaction to the right, with an "apparent" positive flux of $\mathrm{NO}_{2}$ (chemical production during transport) and negative fluxes for $\mathrm{NO}$ and $\mathrm{O}_{3}$. Qualitatively, this seems to correspond to the observations in the canopy. Indeed, a major uncertainty when determining gas fluxes by all methods used in this study is that these methods are based on the assumption of nonreactivity. As has been shown in a number of studies (e.g Kramm et al. 1991; Vilà-Guerau de Arellano and Duynkerke 1992), when chemical reactions have a similar timescale to turbulence, this assumption may lead to erroneous flux calculation. In these cases, the vertical flux becomes a function of height due to chemical production or loss and the flux-profile relationships require modification. The reactive timescale of the interconversion of $\mathrm{O}_{3}-\mathrm{NO}$ and $\mathrm{NO}_{2}$ is in the order of minutes and hence chemical processes are expected to influence the flux calculation (Vilà-Guerau 


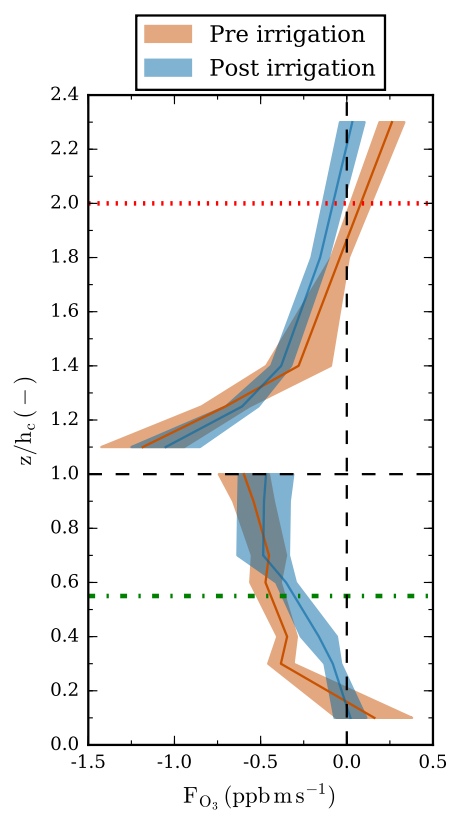

Figure 4.5: Vertical profile of fluxes of $\mathrm{O}_{3}$ calculated via the LOCAL method, and averaged between 10:00 to 17:00 LT for pre- and post-irrigation periods. See Fig. 4.3 for a description of the shaded area and horizontal lines.

de Arellano and Duynkerke 1992; Farmer and Cohen 2008). Kurpius and Goldstein (2003) found that within the canopy chemistry was the dominant $\mathrm{O}_{3}$ loss process during the daytime, with losses of up to $45-55 \%$. The authors suggest that short-living VOCs with a lifetime less than 10 minutes (i.e. monoterpenes) are responsible for the chemical losses of $\mathrm{O}_{3}$ within the canopy. The CHATS measurement set is unfortunately not complete enough to verify this in-canopy chemistry.

\subsubsection{Impact of irrigation on ozone exchange}

As seen in Figs. 4.2 and 4.3, the mixing ratios of $\mathrm{O}_{3}$ show a variation of more than 7 ppb between the pre and post irrigation periods. The overall effect of these processes on inferred $\mathrm{O}_{3}$ fluxes can be seen in Fig. 4.3. There is no significant change in the $\mathrm{O}_{3}$ fluxes pre and post irrigation, except for a slight decrease in fluxes above $20 \mathrm{~m}$ after irrigation.

As a next step, we will investigate reasons for higher pre-irrigation ozone mixing ratios in the boundary layer (Fig. 4.2). As both large-scale and local meteorological conditions vary between pre- and post-irrigation time periods, it is likely that boundary-layer dynamics and/or canopy conditions also contributed to the changes in $\mathrm{O}_{3}$ mixing ratios. We hypothesize the following links between boundary-layer dynamics, canopy conditions, and $\mathrm{O}_{3}$ mixing ratios and fluxes: 


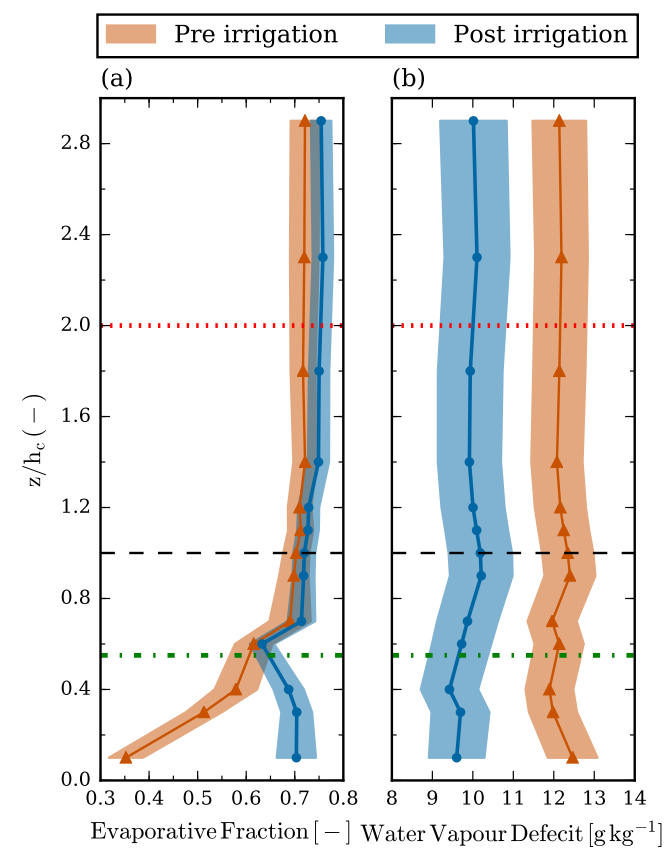

Figure 4.6: Vertical profile of (a) evaporative fraction (EF) and (b) water vapour defecit (WVD) averaged between 10:00 and 17:00 LT for the pre-and post-irrigation period.

i Dry conditions lead to plant stress and the additional production of VOCs, leading to increased $\mathrm{O}_{3}$ chemical production.

ii Dry conditions lead to stomatal closure and decreased deposition of $\mathrm{O}_{3}$, consequently increasing $\mathrm{O}_{3}$ mixing ratios.

iii The differences in $\mathrm{O}_{3}$ mixing ratios pre and post irrigation are mainly depending on the boundary layer thermodynamic conditions (entrainment of ozone from the free troposphere and altered volume of the mixed-layer).

In the following, we investigate these three hypothesis. Firstly, it is necessary to quantify whether drought stress was influencing the soil and vegetation enough to trigger an atmospheric response. Figure 4.6a shows the vertical profile of the evaporative fraction (EF), while Fig. 4.6b shows the water vapour deficit (WVD), both pre and post irrigation. The pre and post irrigation vertical profiles of the EF shows no significant difference within and above the crown. However, a sharp deviation within the canopy can be observed. This difference in EF with irrigation is primarily driven by a strong decrease in latent heat below the canopy $(<5 \mathrm{~m})$ pre irrigation. While the canopy maintains constant water content due to stomatal regulation by the trees and deep roots, 


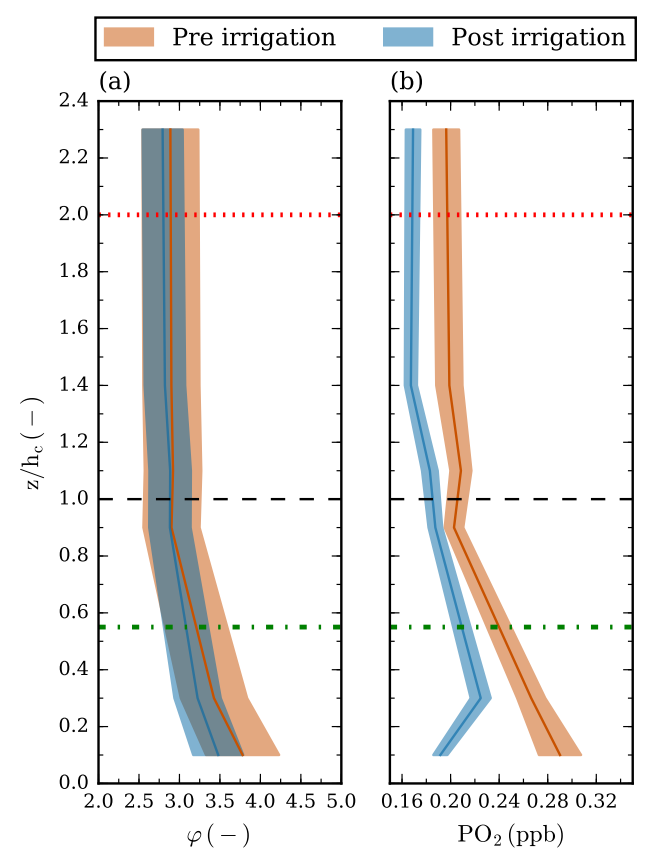

Figure 4.7: Vertical profile of (a) Leighton ratio (PSS) and (b) $\mathrm{PO}_{2}$ averaged between 10:00 and 17:00 LT for the pre-and post-irrigation period.

the soil dries out rapidly, leading to a lower EF within the canopy.

The WVD (Fig. 4.6b) lacks this divergence, likely due to strong mixing throughout the canopy due to canopy sparsity. The observed WVD is similar to values found above a Mediterranean forest (Fares et al. 2014). However, they concluded that stomatal closure due to the high WVD and low soil water content leads to low $\mathrm{O}_{3}$ deposition fluxes. In this study, we find limited evidence for stomatal closure at the CHATS orchard prior to irrigation, since in addition to the lack of difference in EF pre and post irrigation, there was also no significant difference in the average calculated $\mathrm{CO}_{2}$ fluxes (not shown). Due to these two factors, we presume that there are little to no changes in the stomatal deposition of $\mathrm{O}_{3}$ pre and post irrigation.

Dry conditions prior to irrigation may have potentially altered the chemistry of the CHATS site. The change in chemistry conditions was investigated by analysing calculated vertical profiles $\varphi$ and $\mathrm{PO}_{2}$ mixing ratios (Fig. 4.7, see section 4.3.4 for an explanation of $\varphi$ and $\mathrm{PO}_{2}$ ) within and above the canopy. The values of $\varphi$ shows no noteworthy difference pre and post irrigation, and have slightly negative gradients. Even at a height of $23 \mathrm{~m}$ large deviations from PSS are observed, with remaining high at around 3. These high values of $\varphi$ indicate a stronger influence of pathways other than R05 and R19 on the production of $\mathrm{O}_{3}$ pre irrigation. Drivers of these alternate pathways 
are peroxy radicals $\left(\mathrm{PO}_{2}\right)$ which generally result from the oxidation of VOCs or $\mathrm{CO}$ (Griffin et al. 2007). Increased VOC mixing ratios (e.g. Fig. 4.2d and 4.3d) pre irrigation likely led to this increased importance of peroxy radicals in $\mathrm{O}_{3}$ production. The average vertical mixing ratio of PO2, calculated by Eq. 4.14, in Fig. 4.7b is indeed enhanced. Thus the enhanced VOCs emissions and subsequent ozone formation in and above the canopy likely explain the enhanced $\mathrm{O}_{3}$ mixing ratios before irrigation. This would imply that (i) ozone deposition is not strongly influenced by irrigation, and (ii) the enhanced drought-related VOC emissions pre-irrigation do not severely influence $\mathrm{O}_{3}$ fluxes.

Other factors that could drive the difference in concentrations of $\mathrm{O}_{3}$ pre and post irrigation include the emission of NO from soils and surface wetness of soils or leaves (Lamaud et al. 2002). While the irrigation of the CHATS orchard could potentially produce increased fluxes of NO, the calculated NO fluxes did not exhibit enhanced positive fluxes within the canopy post irrigation. In addition, $\mathrm{O}_{3}$ deposition onto soil depends on soil water content and the relative humidity at soil-level, with deposition increasing as these factors increase (Fares et al. 2012). However, as Fig. 4.5 shows, no clear signals of enhanced post-irrigation ozone deposition are found.

Finally, the boundary-layer thermosdynamics can play an important role in the ozone mixing ratio evolution above the canopy. The observed larger differences in $h$ (based on REAL (lidar) data observations (http://lidar.csuchico.edu/lgd/nsf_results)) for pre- and post-irrigation periods (not necessary dependent on the irrigation) could be responsible for the differences in $\mathrm{O}_{3}$ mixing ratio for these periods. Deeper maximum $h$ pre irrigation (reaching roughly around 1200 $\mathrm{m})$ potentially corresponds to entraining more $\mathrm{O}_{3}$ from the free troposphere, leading to larger $\mathrm{O}_{3}$ mixing ratios compared to the $\mathrm{O}_{3}$ mixing ratios post irrigation, when the $h$ remained more shallow (around 700-800 m). Similar holds for the case study day, during which the maximum $h$ was only around $550 \mathrm{~m}$, but also the lowest concentrations of ozone were observed during that day, compared to the average pre and post irrigation $\mathrm{O}_{3}$ mixing ratio (see Figs. 4.2 and 4.4). A larger mixing volume (due to deeper h pre-irrigation) however dilutes the mixing ratios of the precursors for ozone formation, and hence the chemical term in the ozone budget. In short, we cannot a priory draw firm conclusions about the effects of the boundary-layer dynamics on the ozone budget. In section 4.5 we will therefore further quantify the different terms in the ozone budget.

\subsection{Analysis of the diurnal ozone budget}

\subsubsection{Initial and boundary conditions}

In this section, we continue the analysis by calculating the $\mathrm{O}_{3}$ budget (Eq. 4.8) for our case study, Day 147. Each term of the $\mathrm{O} 3$ budget will be dealt with sequentially, starting with the dynamics. As already mentioned in section 4.3.1, we use the MXLCH model (Vilà-Guerau de Arellano et 
al. 2009) to represent and quantify the ozone budget over the CHATS canopy for a selected case study. The reasons to select May 27, 2007 (Day 147) are twofold:

1) Day 147 is the only day in the CHATS data set with dominant winds from south, ensuring a well-developed RSL over the CHATS canopy (the measurement tower is placed at the northern most part of the site) (Patton et al. 2011).

2) The observational dataset is most complete in terms of dynamics and chemistry.

The dynamics of MXLCH were initiated by observations taken at $29 \mathrm{~m}$ on Day 147, with largescale forcing such as subsidence and advection being prescribed (Chapter 3). The model then calculates the temporal evolution of dynamics (temperature, humidity, wind speed and boundary layer height) within the boundary layer. Overall, the modelled state variables were in good agreement with the observations, with dynamics of the case study being well reproduced in MXLCH (Chapter 3).

Now we extend the MXLCH model with chemistry. In the absence of observed $\mathrm{O}_{3}$ profiles during $\mathrm{CHATS}$, the difference in $\mathrm{O}_{3}$ mixing ratio between the free troposphere and the boundary layer $\left(\Delta C_{O_{3}}\right)$ was assumed based on literature. The majority of the observations of ozone profiles over the California Valley were conducted for the purpose of the Central California Ozone Study (CCOS) (Fujita et al. 1999; Fujita et al. 2001; Fujita et al. 2005). Observation of the $\mathrm{O}_{3}$ profiles throughout the boundary layer and the free troposphere showed variable profile shapes, with ozone jumps ranging from around 20 to $40 \mathrm{ppb}$ in the early mornings, 10 to $20 \mathrm{ppb}$ in the late afternoon and even negative ozone jumps of -10 to $-30 \mathrm{ppb}$ in the afternoon and evening (e.g. Fujita et al. 2001). Since the mixed-layer model assumes well-mixed conditions, we selected an initial value of the ozone jump to be around $15 \mathrm{ppb}$ (i.e. $\Delta C_{O_{3}}=15 \mathrm{ppb}$ ). To account for the uncertainty, we performed numerical experiments with variations in $\Delta C_{O_{3}}$ (Table 4.2) and perturb the initial ozone jump by $+/-5 \mathrm{ppb}$. The sensitivity of the $\mathrm{O}_{3}$ budget to these values will be discussed in Sect. 4.5.4.

Concerning ozone deposition, figure 4.8 shows the average velocity deposition $\left(v_{d}\right)$ for $\mathrm{O}_{3}$ for Day 147 calculated by the four different methods within and above the canopy, together with the average for the pre and post irrigation periods. Above and near the canopy top the MOST $+\mathrm{RSL}$ method performs better than the standard MOST method (see appendix 4B). The derived $\mathrm{O}_{3}$ deposition velocity increased towards the top of the canopy, reaching a peak of around $2.8 \mathrm{~cm} \mathrm{~s}^{-1}$ at $10 \mathrm{~m}$ and declining to $0.10 \mathrm{~cm} \mathrm{~s}^{-1}$ at $23 \mathrm{~m}$. These deposition velocities are within the range of values found in other studies (Mikkelsen et al. 2000; Lamaud et al. 2002; Fares et al. 2010). Lamaud et al. (2002) provides a brief overview of $\mathrm{O}_{3}$ deposition velocities above a variety of forest canopies, with the daytime values ranging between 0.25 to $1.8 \mathrm{~cm} \mathrm{~s}^{-1}$. Mikkelsen et al. (2000) found $\mathrm{O}_{3}$ deposition velocity of $0.8 \mathrm{~cm} \mathrm{~s}^{-1}$ above an evergreen forest, while Fares et al. (2010) found $\mathrm{O}_{3}$ deposition velocity of around 0.4 to $0.6 \mathrm{~cm} \mathrm{~s}^{-1}$ above a pine forest. Here, we also note that there is no substantial difference between the pre and post deposition velocities of 

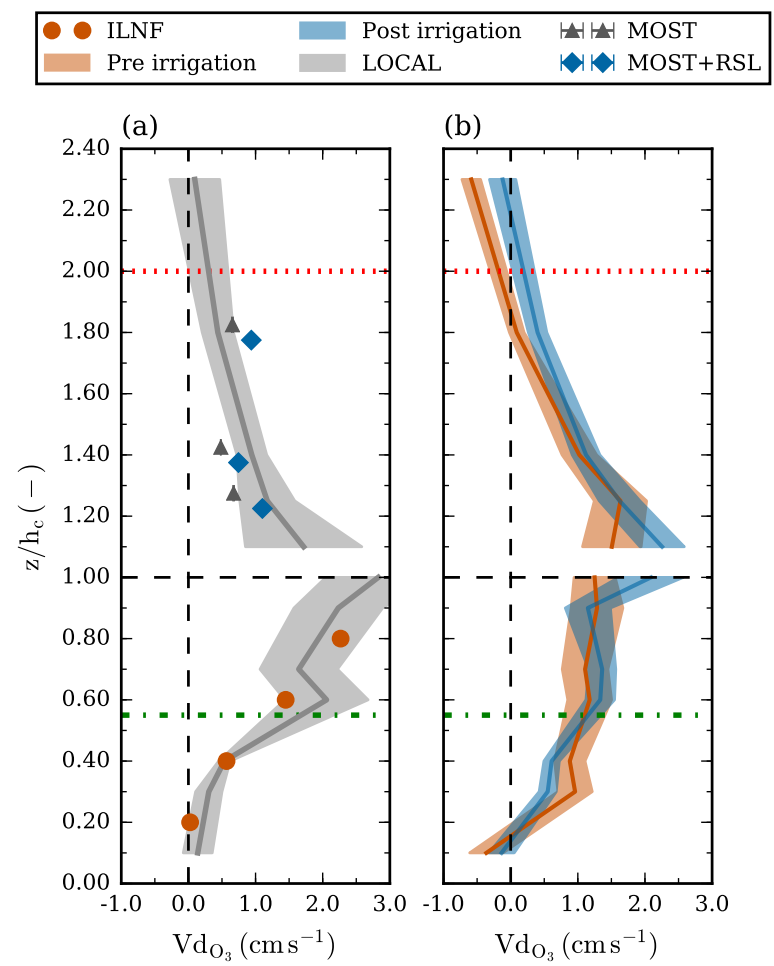

(b)

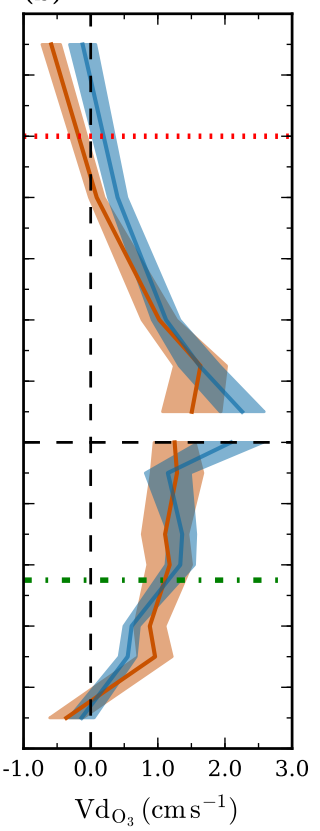

Figure 4.8: Vertical profiles of the deposition velocity of $\mathrm{O}_{3}$ for (a) Day 147 and for (b) the pre and post irrigation periods, calculated via the four different methods and averaged between 10:00 to 17:00 LT. See Fig. 4.3 for a description of the shaded area and horizontal lines.

$\mathrm{O}_{3}$, except for a slight increase at the canopy top and above $18 \mathrm{~m}$ post irrigation (due to difference in ozone mixing ratio). This lack of difference between $\mathrm{O}_{3}$ fluxes and $v_{d}$, between pre and post irrigation, is discussed in Sect. 4.4.3.

We used the $v_{d}$ value calculated by MOST+RSL method at $2.5 \mathrm{~m}$ above the canopy top $(1 \mathrm{~cm}$ $\mathrm{s}^{-1}$ ) (see section 4.3.3 for reasons to choose this height as a reference) and perform a sensitivity using the calculated by MOST $\left(0.5 \mathrm{~cm} \mathrm{~s}^{-1}\right.$, see Table 4.2). Concerning $\mathrm{NO}_{x}$, Fig. 4.4 shows that $\mathrm{NO}$ and $\mathrm{NO}_{2}$ fluxes shifted from negative above the canopy (12.5 m, indicative of deposition) to positive at $23 \mathrm{~m}$, possibly indicating advection of $\mathrm{NO}_{x}$ from the nearby highway in the boundary layer. The NO deposition velocity was calculated with MOST and MOST+RSL as 1 and 2 $\mathrm{cm} \mathrm{s}^{-1}$, respectively. Deposition velocities for $\mathrm{NO}_{2}$ were 0.22 and $0.28 \mathrm{~cm} \mathrm{~s}^{-1}$ for MOST and MOST+RSL, respectively (Table 4.2). To maintain the high $\mathrm{NO}_{x}$ mixing ratios observed during the day (Figs. 4.2 and 4.3), we assume advection of $\mathrm{NO}_{2}$ over the CHATS site (Table 4.2) (assuming that all $\mathrm{NO}$ is already converted to $\mathrm{NO}_{2}$ ). 
Table 4.2: Initial and boundary conditions used in the MXLCH numerical experiment ('Control') with the chemistry scheme listed and described in Table 4.1. The $t$ is the elapsed time since the start of the experiment and $t_{d}$ is the time (is seconds) duration of the experiment (from 08:00 17:00 LT). CO mixing ratios were taken from California Environmental Protection Agency Air Resources Board air quality data (http://www. arb.ca.gov/aqmis2/aqdselect.php).

\begin{tabular}{l|rrrrrr}
\hline \hline Experiment: Control & $\mathrm{O}_{3}$ & $\mathrm{NO}$ & $\mathrm{NO}_{2}$ & $\mathrm{ISO}$ & $\mathrm{CH}_{2} \mathrm{O}$ & $\mathrm{CO}$ \\
\hline$\left\langle C_{0}\right\rangle[\mathrm{ppm}]$ & 21 & 0.6 & 3.5 & 0 & 1 & 200 \\
$\Delta C=C_{F T}-\left\langle C_{0}\right\rangle[\mathrm{ppm}]$ & $15^{\mathrm{a}}$ & -0.6 & -2.5 & 0 & 0 & 0 \\
$F_{O_{3}}\left[\mathrm{ppb} \mathrm{m} \mathrm{s}{ }^{-1}\right]$ & $/$ & $/$ & $/$ & $\mathrm{b}$ & 0 & 0 \\
$v_{d}\left[\mathrm{~cm} \mathrm{~s}^{-1}\right]$ & MOST+RSL: 1 & 2 & 0.28 & $/$ & $/$ & $/$ \\
& MOST: 0.5 & 1 & 0.22 & $/$ & $/$ & $/$ \\
Advection $\left[\mathrm{ppt} \mathrm{s}^{-1}\right]$ & -0.7 & 0 & 0.5 & 0 & 0 & 0 \\
\hline
\end{tabular}

${ }^{\mathrm{a}}$ If not specified differently (see Sect. 4.5.3)

${ }^{\mathrm{b}} F_{I S I}=0.0005 \sin \left(\pi t / t_{t}\right)$, If not specified differently (see Sect. 4.5.3)

We model VOCs by including a simplified scheme for isoprene oxidation and assume a diurnal cycle in its emissions to simulate its radiation dependence (Table 4.2). Note again that observed isoprene mixing ratios were mainly low (Thomas Karl, personal communication) and that little information is available concerning the abundance of natural and anthropogenic VOC species. We assume initial mixing ratios of $200 \mathrm{ppb}$ and $1800 \mathrm{ppb}$ of $\mathrm{CO}$ and $\mathrm{CH}_{4}$, respectively, to provide hydrocarbon species that produce $\mathrm{RO} 2$ radicals that account for ozone formation through $\mathrm{RO}_{2}+$ $\mathrm{NO} \rightarrow \mathrm{NO}_{2}+\mathrm{RO}(\mathrm{R} 13)$ followed by $\mathrm{NO}_{2}$ photolysis (R05).

\subsubsection{The effects of the roughness sublayer}

Figure 4.9 shows the modelled temporal variation of the mixed-layer $\mathrm{O}_{3}$ and $\mathrm{NO}_{x}$ mixing ratio as compared to the observed $\mathrm{O}_{3}$ and $\mathrm{NO}_{x}$ mixing ratio at $23 \mathrm{~m}$ above the ground surface (at the highest measurement level). It appears that in this model configuration with limited VOCs, ozone formation is rather limited because of the high $\mathrm{NO}_{x}$ and low VOC regime, which means that the path $\mathrm{OH}+\mathrm{NO}_{2} \rightarrow \mathrm{HNO}_{3}$ is important relative to $\mathrm{OH}+$ hydrocarbon $\left(\mathrm{CO}, \mathrm{CH}_{4}\right.$, ISO, other hydrocarbons). Increasing the emissions or abundance of hydrocarbons under these conditions would boost $\mathrm{O}_{3}$ production, requiring an outflux of $\mathrm{O}_{3}$ to stay in line with the observations (see additional analysis in Section 4.5.3).

Figure 4.9 also shows the effect of the RSL on the $\mathrm{O}_{3}$ and the NOx mixing ratio temporal variation. Important and relevant for this study is that a $50 \%$ decrease in ozone deposition velocity (MOST) leads to on average $16 \%$ higher $\mathrm{O}_{3}$ mixing ratios. Due to feedbacks in the chemistry, $\mathrm{NO}_{x}$ mixing ratios decreases by $9 \%$ at the end of the simulation period (Fig. $4.9 \mathrm{~b}$ ). 

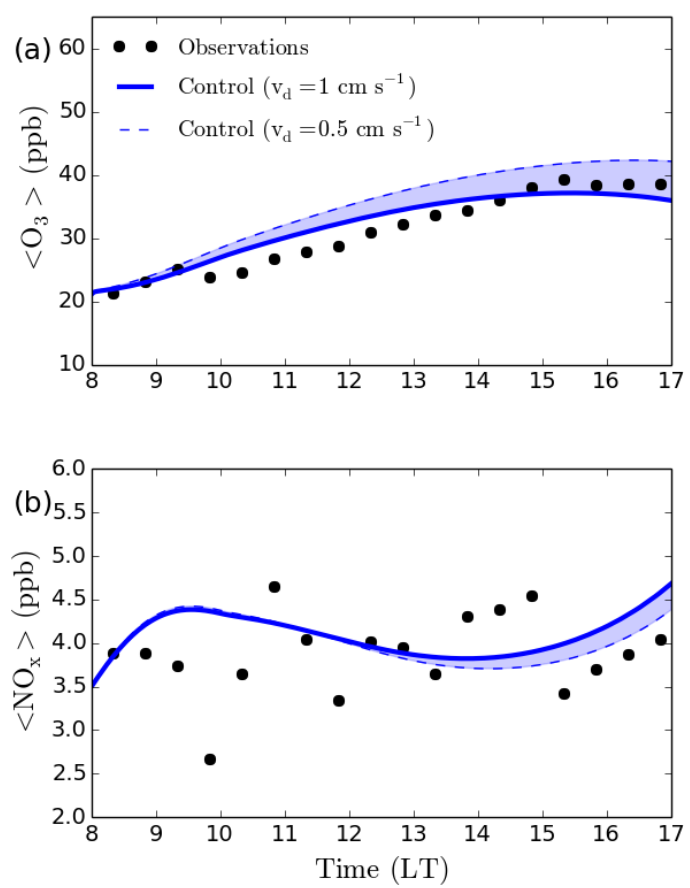

Figure 4.9: Temporal variation of the mixed-layer ozone (a) and $\mathrm{NO}_{x}\left(=\mathrm{NO}+\mathrm{NO}_{2}\right)(\boldsymbol{b})$ mixing ratios over the CHATS canopy. Black full circles represent the observed concentrations at the highest measurement level (23 $\mathrm{m}$ above the ground surface). The blue solid line represents the $M X L C H$ experiment (Control) with RSL effects accounted for in deposition velocity calculation $\left(M O S T+R S L: v_{d}=1 \mathrm{~cm} \mathrm{~s}^{-1}\right)$. The dashed line shows the mixed-layer model experiment (Control) with standard MOST deposition velocity calculation (MOST: $v_{d}=0.5 \mathrm{~cm} \mathrm{~s}^{-1}$ ). Initial and boundary conditions are presented in Table 4.2.

\subsubsection{Sensitivity of simulated $\mathrm{O}_{3}$ and $\mathrm{NO}_{x}$}

Here, we investigate the sensitivity of the model results to the assumptions we made in our numerical experiment for the parameterization of the ozone entrainment flux and the surface emission of hydrocarbons (in our case isoprene), as well as the role of ozone and $\mathrm{NO}_{x}$ advection.

Our standard run (Control) assumes a jump of the ozone-mixing ratio in the entrainment zone of $15 \mathrm{ppb}$ (Table 4.2). As already mentioned in section 4.5.1, a realistic initialization of the value of $\Delta C_{O_{3}}$ in the model experiment remains uncertain due to the lack of $\mathrm{O}_{3}$ profile observations. Therefore, and based on literature (Fujita et al. 1999; Fujita et al. 2001; Fujita et al. 2005), we performed additional numerical experiments with different ozone jumps (10 and $20 \mathrm{ppb}$ ). Figure 4.10a and 4.10c shows the sensitivity of the diurnal variation of $\mathrm{O}_{3}$ and $\mathrm{NO}_{x}$ for an initial 20 

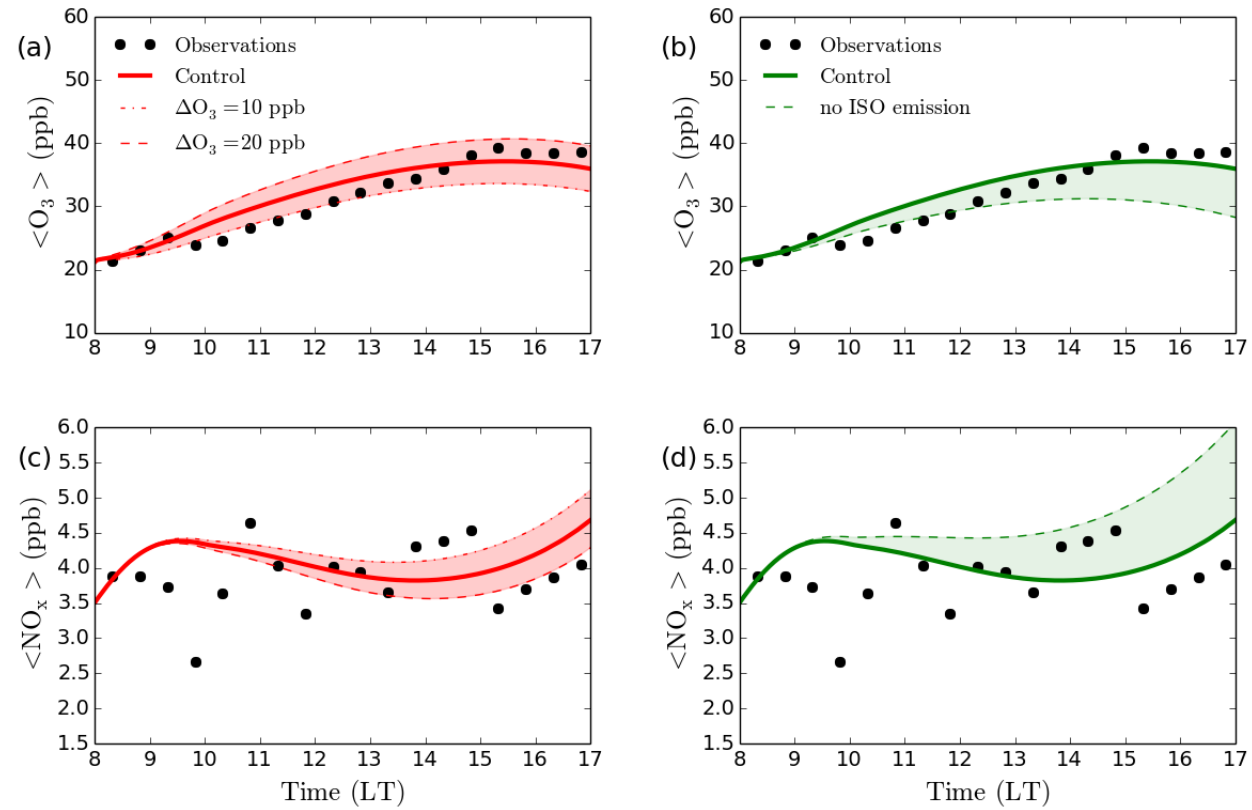

Figure 4.10: Temporal variation of the ozone ( $\boldsymbol{a}$ and $\boldsymbol{b})$ and $N O_{x}(\boldsymbol{c}$ and $\boldsymbol{d})$ mixing ratios over the CHATS canopy. Black full circles represent the observed mixing ratios at the highest measurement level (23 $m$ above the ground surface). The red and green solid lines represent the MXLCH experiment 'Control' with the settings for the entrainment and isoprene emission as in Table 4.2; the dashed and dash-dotted red lines show the sensitivity to varying the initial ozone jump and green dashed lines depict the results for no isoprene emission, respectively. All other initial and boundary conditions are similar as in 'Control' (Table 4.2).

ppb (dashed red line) and $10 \mathrm{ppb}$ (dash-dotted red line) ozone jump at the entrainment zone. As expected, the high ozone jump leads to an increased $\mathrm{O}_{3}$ mixing ratio in the boundary layer because ozone rich air entrains the mixed-layer from the overlying atmosphere. The opposite holds for the $10 \mathrm{ppb}$ ozone jump. Effects on $\mathrm{NO}_{x}$ are opposite, with higher ozone shortening the $\mathrm{NO}_{x}$ chemical lifetime.

The results presented until now included the surface emission of isoprene (Table 4.2). Figure $4.10 \mathrm{~b}$ shows the effects of not including any isoprene emission on the mixed-layer $\mathrm{O}_{3}$ and $\mathrm{NO}_{x}$ mixing ratios (Fig. 4.10d). As expected, decreasing the emission of isoprene in this VOC limited regime leads to higher $\mathrm{NO}_{x}$ and lower $\mathrm{O}_{3}$ mixing ratios (Fig. 4.10b), since VOC is precursor of ozone and depletes $\mathrm{NO}_{x}$ (see also Sillman 1999).

Although there is a satisfactory agreement in values and diurnal variations of the modelled and the observed $\mathrm{O}_{3}$ and $\mathrm{NO}_{x}$ mixing ratios (Fig. 4.9), the model results for the $\mathrm{NO}_{2} / \mathrm{NO}$ ratio, based 

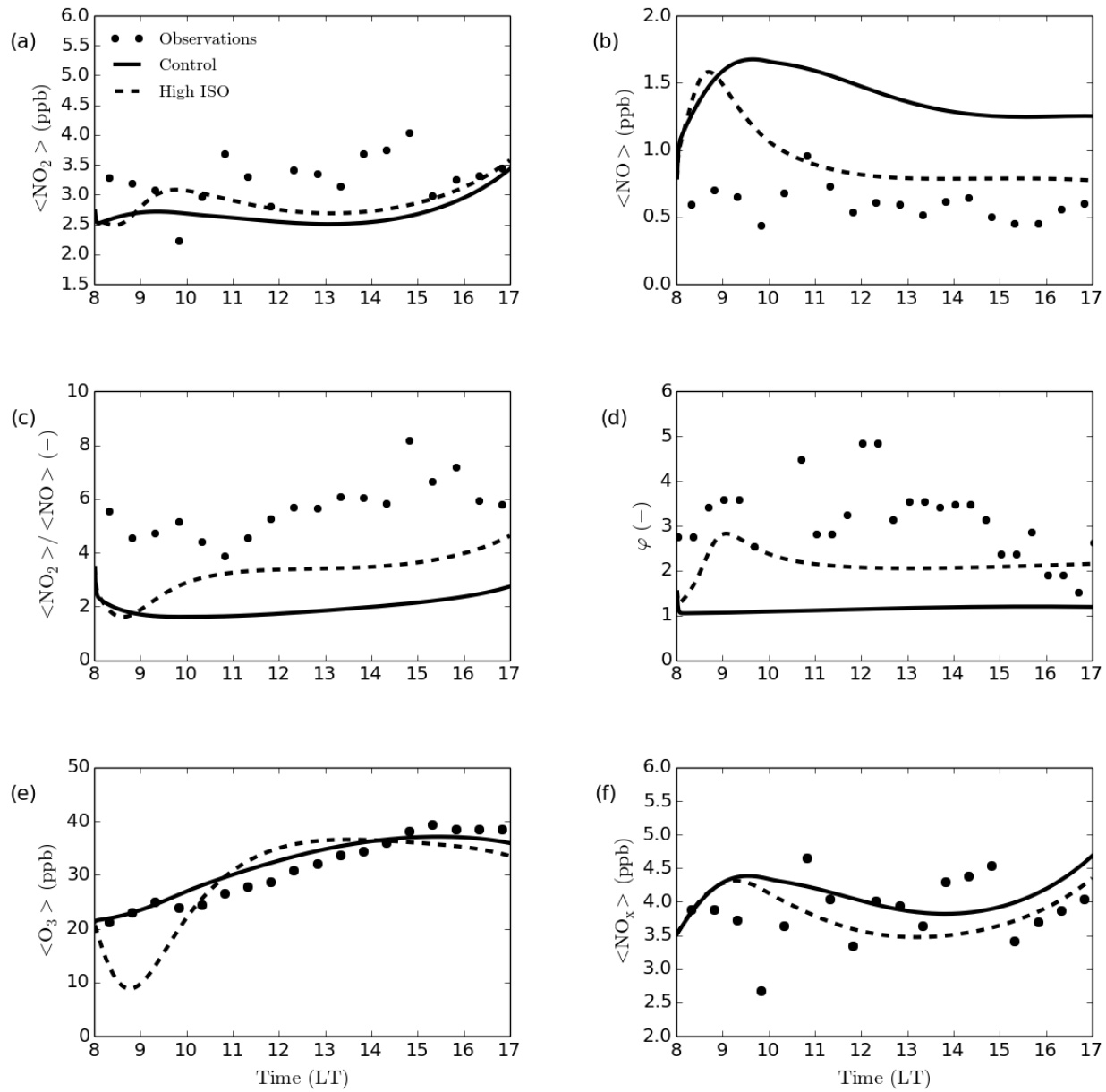

Figure 4.11: Temporal variation of the observed and modelled (a) $\mathrm{NO}_{2}$, (b) $\mathrm{NO}$, (c) $\mathrm{NO}_{2} / \mathrm{NO},(\boldsymbol{d})$ Leighton ratio (PSS), (e) $\mathrm{O}_{3}$, and (f) $\mathrm{NO}_{x}$ for the case study (147 DOY). Solid lines represent the model based on initial and boundary condition for numerical experiment 'Control' (Table 4.2), while dashed lines illustrate the results of the numerical experiment 'High ISO' (Table 4.3) with increased VOCs (represented by ISO) emissions and altered advection of ozone and $\mathrm{NO}_{2}$. 
Table 4.3: Similar as in Table 4.2, but here only modifications compared to the 'Control' experiment are presented.

\begin{tabular}{l|rrrrrr}
\hline \hline Experiment: High ISO & $\mathrm{O}_{3}$ & $\mathrm{NO}$ & $\mathrm{NO}_{2}$ & ISO & $\mathrm{CH}_{2} \mathrm{O}$ & $\mathrm{CO}$ \\
\hline$\left\langle C_{0}\right\rangle[\mathrm{ppm}]$ & & 0 & & & & 400 \\
$\Delta C=C_{F T}-\left\langle C_{0}\right\rangle[\mathrm{ppm}]$ & & & & & & 0 \\
$F_{O_{3}}\left[\mathrm{ppb} \mathrm{m} \mathrm{s}{ }^{-1}\right]$ & & & & & 1.5 & 0 \\
Advection [ppt s$\left.{ }^{-1}\right]$ & -9.5 & 0 & 0.8 & 0 & 0 & 0 \\
\hline
\end{tabular}

on the settings of the numerical experiment 'Control' (Table 4.2), show a severe underestimation compared to the observations (Fig. 4.11a,b,c). To account for this underestimation of the $\mathrm{NO}_{2} / \mathrm{NO}$ ratio, we set up a new numerical experiment ('High ISO', Table 4.3) in which we increase the natural (generic) VOCs emissions by increasing the isoprene emissions and the anthropogenic VOC abundance by increasing the initial CO mixing ratio. The aim here is to increase $\mathrm{PO}_{2}($ based on the PSS, Fig. 4.11d) and to trigger more efficient conversion of $\mathrm{NO}$ to $\mathrm{NO}_{2}$.

Since the increased $\mathrm{NO}_{2}$ leads to increased $\mathrm{O}_{3}$ (due to $\mathrm{NO}_{2}$ photolysis), we invoked an outflow flux of ozone by advection (Table 4.3) to reduce the ozone mixing ratio in the boundary layer to the level of the observed ozone mixing ratio (Fig. 4.11e). This negative ozone advection is therefore constrained by the observations. By doing so, we managed to improve the $\mathrm{NO}_{2} / \mathrm{NO}$ (Fig. 4.11c) representation and yet keeping the low ozone conditions as observed. The magnitudes of the added chemistry and advection to the system are discussed in section 4.5.4. Unfortunately, given the scarcity of especially VOC data, we cannot conclude whether our 'High ISO' simulation is realistic. However, we note that the production of $\mathrm{O}_{3}$ is much higher in 'High ISO' compared to 'Control', and that the simulated diurnal $\mathrm{O}_{3}$ variations reproduce the observations slightly worse. Note that the peak at the beginning of the 'High ISO' experiment for $\mathrm{NO}_{2}, \mathrm{NO}$ and $\mathrm{O}_{3}$ is due to the large increase of the isoprene mixing ratio after its zero initialization due to the 'high' emission. Further adjustments are possible to remove remaining discrepancies, but little observational evidence is available to infer variable advection and/or emissions of the chemical species. Simulations with a meso-scale chemistry transport model would be required to better quantify advection of ozone and its precursors over the CHATS site.

\subsubsection{Budgeting of ozone}

Based on the provided modelling results and to complete the study, we perform an analysis on the individual contributions from deposition, entrainment, chemistry and advection to the diurnal variation of $\mathrm{O}_{3}$, including a sensitivity analysis. The ozone budget equation reads: 


$$
\underbrace{\frac{\partial C_{O_{3}}}{\partial t}}_{\text {Total }}=\underbrace{\frac{\partial\left(\overline{w^{\prime} C_{O_{3}}^{\prime}}\right)_{s}}{h}}_{\text {Deposition }}-\underbrace{\frac{\partial\left(\overline{w^{\prime} C_{O_{3}}^{\prime}}\right)_{e}}{h}}_{\text {Entrainment }}+\underbrace{S_{O_{3}}}_{\text {Chemistry }}-\underbrace{\left(u \frac{\partial C_{O_{3}}}{\partial x}+v \frac{\partial C_{O_{3}}}{\partial y}\right)}_{\text {Advection }} .
$$

Figure 4.12 shows the contributions of deposition, entrainment, and chemistry to the total ozone tendencies as calculated with MXLCH (Eq. 4.15). We show the tendencies of the individual contributors to the total ozone budget for the standard numerical experiment 'Control', but also include results from the sensitivity experiment 'High ISO' described in section 4.5.4.

Since the deposition velocity of ozone is taken constant (assuming well-mixed conditions (see section 4.3.3)) during the day, the influence of deposition on the $\mathrm{O}_{3}$ tendency depends on the boundary layer evolution. In the early morning, when the boundary layer is still shallow, the impact of deposition is largest. Logically, the smaller deposition velocity (MOST) leads to a smaller negative contribution to the ozone tendency (Fig. 4.9a). The entrainment contribution to the total ozone budget is positive, mainly depending on the magnitude of the ozone jump as well as the entrainment velocity $\left(w_{e}\right)$ (see Eq. 4.9). Entrainment influences the $\mathrm{O}_{3}$ tendency mainly in the morning when the boundary layer grow fast. At the end of the day, the entrainment contribution becomes smaller, because the $\mathrm{O}_{3}$ mixing ratios in the boundary layer exceed the values in the overlying atmosphere. Note that these results of the entrainment contribution are very sensitive to the choice in the ozone jump (Fig. 10a). The presented spread in Fig. 10a is based on only a small variation of the ozone jump ( $\pm 5 \mathrm{ppb})$. Chemistry is diagnosed to be a source of ozone with a maximum magnitude in the afternoon due to its dependence on solar radiation and the $\mathrm{NO}_{x}$ and hydrocarbon precursor mixing ratios. Related to this, the effect of not including the isoprene emission reduces the contribution of the chemistry to the total budget of ozone by more than $50 \%$ around noon (not shown). The increased chemical activity, due to increased VOCs in the numerical experiment 'High ISO' to account for the underestimated $\mathrm{NO}_{2} / \mathrm{NO}$ mixing ratio in 'Control', leads to a large production of ozone of up to $40 \mathrm{ppb} \mathrm{h}^{-1}$ (Fig. 4.12b). In order to keep the modelled ozone in agreement with the observations (Fig. 4.11e), we invoked an advection of ozone of $-2.2 \mathrm{ppb} \mathrm{h}^{-1}$ in the experiment 'Control', which is similar in magnitude as deposition (Fig. 4.12a). This advection increases to up to $-33 \mathrm{ppb} \mathrm{h}^{-1}$ in experiment 'High ISO' to account for the large ozone production.

As a summary, for the 'Control' experiment each of the individual terms in Eq. 4.15 has a significant and important contribution to the total ozone budget. Our analysis implies that chemistry and advection play a dominant role in the ozone budget over CHATS if we reproduce the $\mathrm{NO}_{2} / \mathrm{NO}$ observations by invoking large $\mathrm{PO}_{2}$ mixing ratios. 

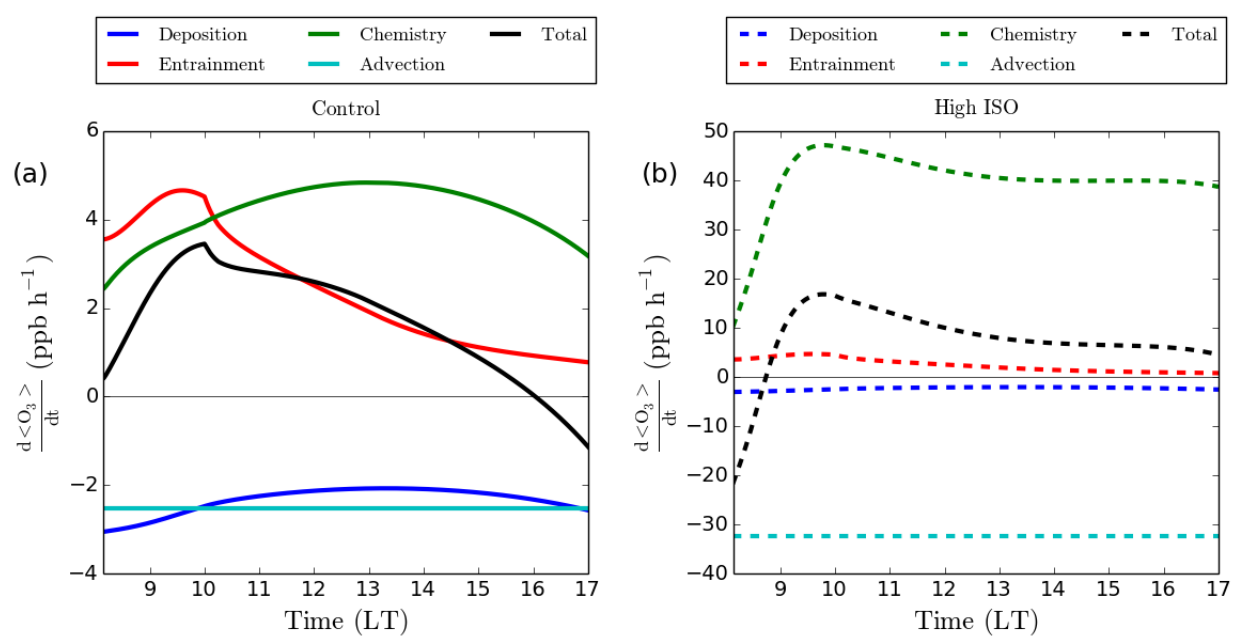

Figure 4.12: Tendencies of the individual terms in the ozone budget as defined in Eq. 4.15 for the two numerical experiment (a) 'Control', and (b) 'High ISO' as initialized in Table 4.2 and 4.3. The solid (Control) and dashed (High ISO) colored lines represent the contributions of the different terms (Eq. 4.15).

\subsection{Conclusions}

We investigated the ozone $\left(\mathrm{O}_{3}\right)$ diurnal variability above an orchard canopy, placing special emphasis on the canopy effects on deposition of ozone. The deposition contribution on ozone variability has been estimated from vertical profiles of ozone measurements, combined with measurements of the relevant micrometeorological and turbulent quantities. The study combined available observations from the Canopy Horizontal Array Turbulence Study (CHATS) with conceptual modelling using an atmospheric chemistry mixed-layer model (MXLCH), and aimed to obtain a balanced description of surface, dynamics and chemical contributions to the temporal variability of ozone.

A general characterization of key chemical species during CHATS is presented, in terms of temporal evolution and vertical profiles. Relatively low maximal values of ozone with respect to Central California Valley values are found (lower than 40 - $60 \mathrm{ppb}$ ) with small differences between pre- and post-irrigation periods. Observed differences in the mixing ratios of $\mathrm{O}_{3}$ pre and post irrigation were likely due to the combined effects of dynamics and emissions of biologically emitted VOCs. An indication for the latter is that chemical composition of the CHATS boundary layer showed very strong deviations from the photostationary state, with a Leighton ratio of up to 3. This likely indicates a strong influence of other chemical reactions converting $\mathrm{NO}$ to $\mathrm{NO}_{2}$ and leading to production of $\mathrm{O}_{3}$ during the day. The observed lower ozone-mixing ratio over CHATS 
for the selected case study therefore required a strong outflow of ozone.

Surface exchange of gases was calculated using four methods to retrieve vertical fluxes from observed mean mixing ratios within and above the canopy. All methods gave relatively similar results for $\mathrm{O}_{3}, \mathrm{NO}$ and $\mathrm{NO}_{2}$. Above and near the canopy top, however, the flux-gradient method accounting for the roughness sublayer (RSL) effects led to ozone deposition fluxes that were $50 \%$ larger compared to those calculated by the flux-gradient method based on the traditional similarity theory. Consequently, the derived daily average deposition velocity for ozone was $50 \%$ larger when the RSL effects in the flux parameterization was included. Applying this difference in the diurnal mixed-layer ozone calculation resulted in a $16 \%$ decrease in ozone mixing ratios (daily average).

By studying the individual contributions of deposition, entrainment, advection, and chemical production or loss to the ozone budget for our case study, we found that the chemistry has the largest contribution to the total ozone budget, but the deposition and the entrainment were also of relevant importance. The sensitivity of the $\mathrm{O}_{3}$ budget to the range of uncertainties related to the initialization of the ozone jump (upper model boundary), advection processes and the hydrocarbons emissions (e.g. isoprene) was relatively important for our case study. This highlights the need for future experiments to perform more detailed observations of vertical profiles of reactive species such as VOCs, $\mathrm{OH}, \mathrm{RO}_{2}$ and $\mathrm{HO}_{2}$ throughout the boundary layer, to better understand the interactions between dynamics and chemistry over tall canopies. Also, to better quantify the dynamical behavior of ozone and its chemical precursors, simulations and observations over a wider region are required. 


\section{$\Vdash=$ MOST $\leftrightarrow$ MOST + RSL $O$ O Observations}
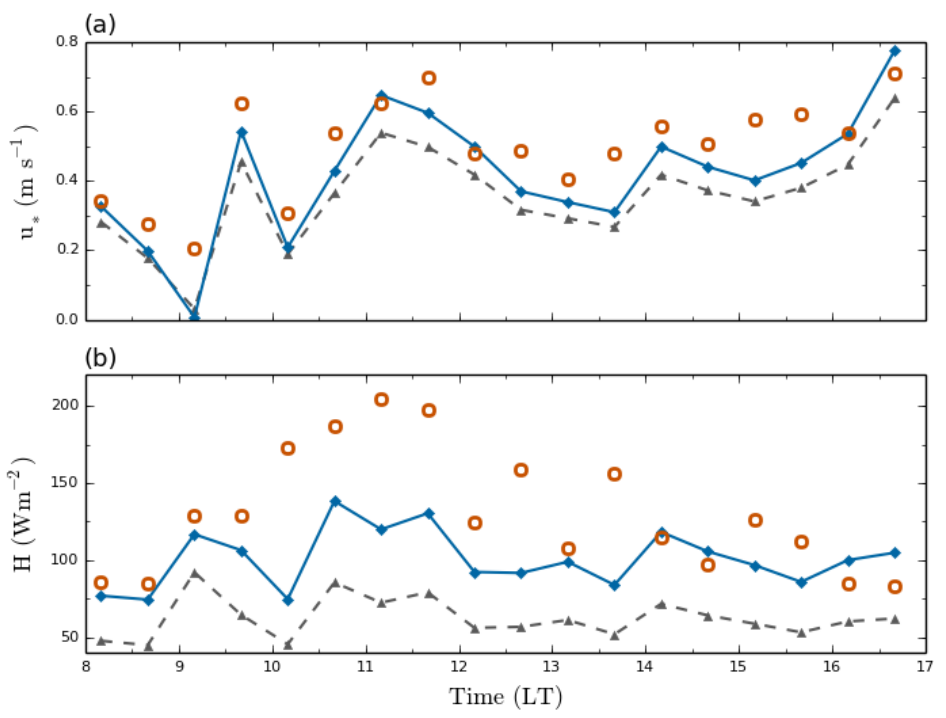

Figure 4A.1: Observed and calculated (by MOST and MOST+RSL methods (Section 4.2.2)) temporal evolution of $(\boldsymbol{a})$ friction velocity $\left(u_{*}\right)$ and $(\boldsymbol{b})$ sensible heat flux $(H)$ on Day 147 between 08:00 to 17:00 LT.

\section{A Appendix: Heat flux and friction velocity above and within the canopy}

To determine the accuracy of the flux calculation methods (discussed in section 4.2.2), we verify here the calculations against observations. We investigate the validity of both the MOST and the MOST + RSL methods above the canopy and the ILNF method within the canopy by comparing calculated and observed friction velocity and heat fluxes.

Above the canopy The temporal evolution of observed and calculated $u_{*}$ and $\mathrm{H}$ for Day 147, calculated by the MOST and MOST+RSL methods at $12.5 \mathrm{~m}$ above the ground surface is presented in Fig. 4A.1. $u_{*}$ and $\mathrm{H}$ are calculated from the observed mean wind and potential temperature at $11 \mathrm{~m}$ and $14 \mathrm{~m}$ above the ground surface by using the flux retrieval method from two levels by Moene and van Dam (2014) (see Section 4.2.2). As previously shown (Molder 1999; De Ridder 2010), within the roughness sublayer the fluxes calculated by the MOST+RSL method reproduce the observations better than MOST. For our case study, the calculated $u_{*}$ reproduces the observations well, while the agreement for $\mathrm{H}$ is less good. It is clear however, that for $\mathrm{H}$ the MOST+RSL method provides a more accurate parameterization of the flux than the MOST method when compared with the eddy covariance observations (Fig. 4A.1b). 

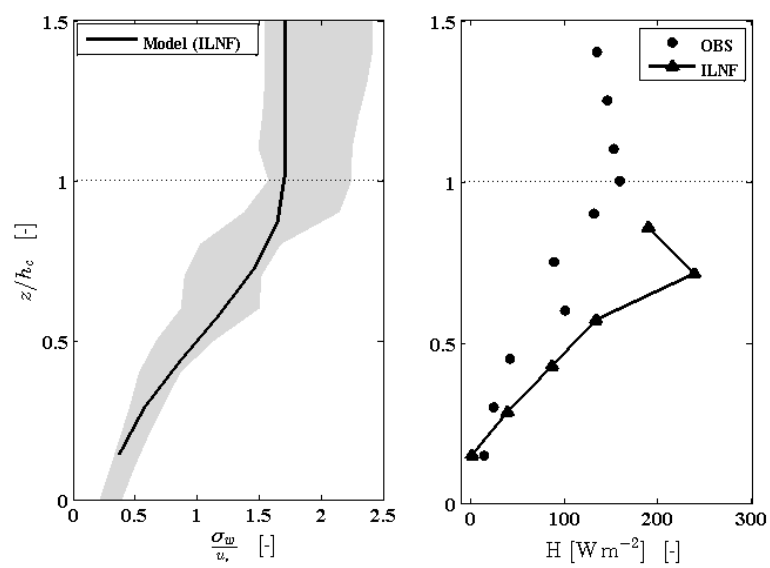

Figure 4A.2: Average profiles of (a) vertical velocity standard deviation $\left(\sigma_{w}\right)$, normalized with the average friction velocity $\left(u_{*}\right)$, and $(\boldsymbol{b})$ sensible heat flux $(H)$, calculated with the Inversed Localized Near-Field (ILNF) method (see Section 4.2.2) for Day 147. Profiles are averaged between 08:00 to 17:00 LT.

Within the canopy Figure 4A.2 shows the performance of the Inverse Localized Near-Field method (Raupach 1989a) in calculating the vertical heat fluxes (sensible heat in our case), with the vertical velocity variance and potential temperature profiles from the CHATS observations.

The model captures the trend of the sensible flux within the canopy, but overestimates the magnitude. The overestimation of $\mathrm{H}$ is related with the fact that the applied model did not consider advection of heat and moisture as shown in Chapter 3. In this chapter is also shown that the observed surface energy balance for Day 147 is not closed and the $30 \%$ lack of energy is related to the measured sensible and latent heat fluxes. Based on that, we can conclude that the model overestimation in Fig. 4A.2 is likely due to energy that is not measured, and that the model produces reasonable results based on the observed potential temperature profiles.

\section{B Appendix: Parameterizing ozone fluxes above CHATS canopy}

Figure 4B.1a shows the diurnal variation of the ozone fluxes calculated by LOCAL, MOST and MOST+RSL methods (see section 4.2.2) at $12.5 \mathrm{~m}$. The fluxes are calculated from the measured ozone mixing ratios at 11 and $14 \mathrm{~m}$ using the flux retrieval procedure explained in Moene and van Dam (2014). The diurnal evolution of the ozone flux for the given case study shows irregular patterns (changing sign), with and daily maximum deposition in the afternoon (between 13:00 and 15:00 LT).

These irregular patterns are probably related to non-stationary conditions that makes 30- 

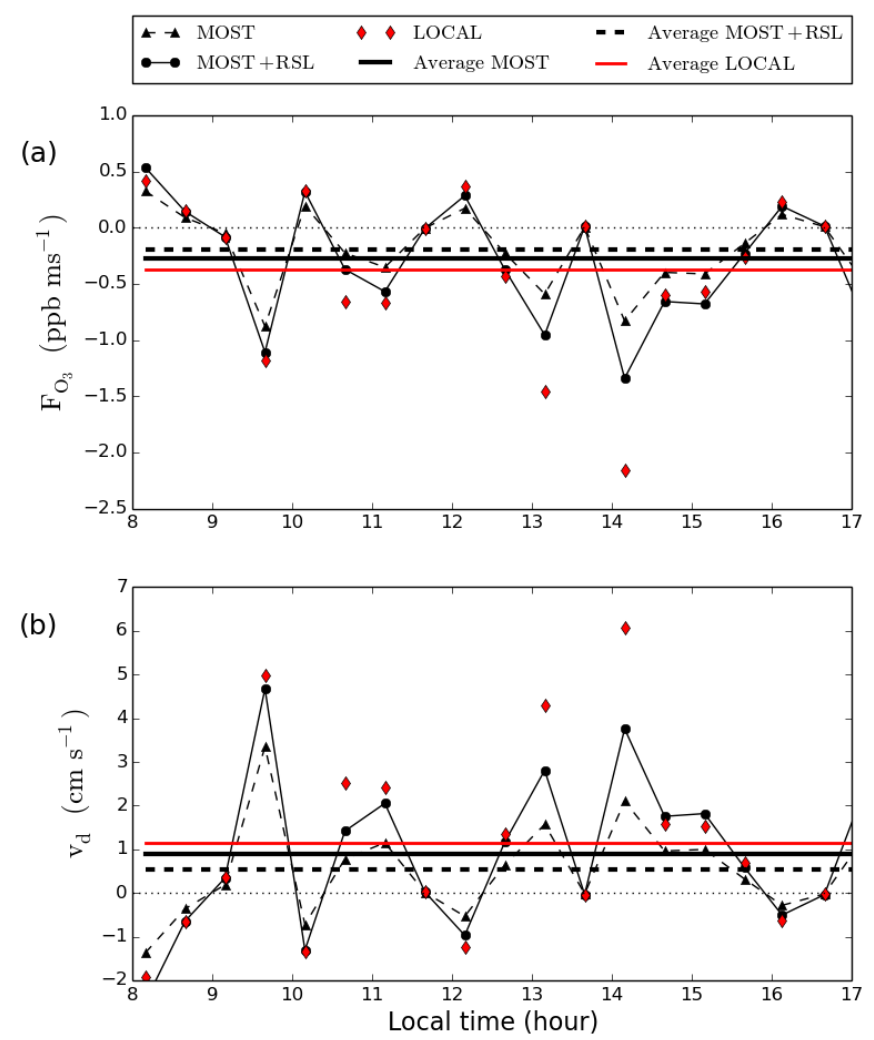

Figure 4B.1: Temporal evolution and daily average of (a) ozone fluxes and (b) deposition velocities at $2.5 \mathrm{~m}$ above the canopy (12.5 $\mathrm{m}$ above the ground surface) on Day 147 between 08:00 to 17:00 LT.

minute averages instable. Similar as for the sensible heat (Fig. 4A.1), the MOST+RSL is more consistent with the observations (LOCAL) than the standard MOST method. This is quantified by the daily averaged fluxes, which are $-0.42,-0.35$ and $-0.19 \mathrm{ppb} \mathrm{m} \mathrm{s}^{-1}$ for LOCAL, MOST, and MOST+RSL respectively (see horizontal lines in Fig. 4B.1).

Figure 4B.1b presents the diurnal variation in the ozone deposition velocity as calculated from Eq. 4.11 and using the fluxes derived with LOCAL, MOST and MOST+RSL and the observed evolution of the ozone-mixing at the highest level above the canopy. Although the diurnal evolution of the deposition velocity often shows positive and negative values (meaning deposition and emission of ozone), the daily average (and especially under well-mixed conditions) shows positive values for all methods (deposition of ozone). We note that the negative values in the deposition 
velocity are unphysical and result of the calculations using the applied method. As already mentioned, the irregular diurnal patterns are likely due to averaging (30 minutes) too short to capture the dynamic behavior of the ozone exchange between the canopy and the overlying atmosphere. Again, the MOST+RSL method is more consistent magnitude than the standard MOST when compared to the LOCAL method. 


\title{
General discussion and future recommendations
}

\begin{abstract}
In this chapter, we discuss our findings on the exchange of energy, momentum and reactive compounds between high vegetation and the atmosphere in a broader perspective by connecting and relating them to previous research in this field. Based on the findings and the gained experience during this thesis, we provide recommendations for future studies. Here, we mainly focus on how this current investigation can continue by adding conditions and mechanisms, which are potentially important for the subject. More specifically, and extending on our results presented at Chapters 2, 3 and 4, we focus on the role of canopy sparsity, the impact of chemical transformations for emitted or deposited reactive compounds near the canopy, as well as the influence of non-local atmospheric phenomena in interpreting measurements and modelling above the canopy.
\end{abstract}

Our discussion points are anchored by the conservation equation of a generic variable, which is applied above high vegetation (e.g. forests, orchards, vineyards, cornfields). The temporal evolution of boundary-layer (thermo)dynamic variables (e.g. $\varphi \equiv U, \theta, q$ ) and atmospheric reactive constituents (e.g. $\varphi \equiv\left[\mathrm{O}_{3}\right],[\mathrm{NO}],\left[\mathrm{NO}_{2}\right]$ ) above the Earth's surface reads (e.g., Jacobson 2005; Stull 2009; Seinfeld and Pandis 2016):

$$
\underbrace{\frac{\partial \varphi}{\partial t}}_{\mathrm{I}}=\underbrace{-\frac{\partial \overline{w^{\prime} \varphi^{\prime}}}{\partial z}}_{\mathrm{II}}+\underbrace{S_{\varphi}}_{\mathrm{III}}-\underbrace{\left(u \frac{\partial \varphi}{\partial x}+v \frac{\partial \varphi}{\partial y}\right)}_{\mathrm{IV}} .
$$


Equation 5.1 quantifies the variation of the variable $\varphi$ under consideration over time $\varphi(z, t)(\mathrm{I})$, which depends on the divergence of the turbulent flux (II), the source/sink vertical distribution of (III), and advective processes (IV). Equation (5.1) encapsulates all the processes treated in this thesis and helps us to frame the discussion.

As introduced in Chapters 1 and 2, the most common way to parameterize the turbulent fluxes (II) in atmospheric models (Eq. (5.1)) is to relate them to the corresponding mean gradients using the turbulent exchange coefficient of $\varphi, K_{\varphi}$ (known as $K$-theory) (e.g. Kaimal and Finnigan 1994):

$$
\overline{w^{\prime} \varphi^{\prime}}=-K_{\varphi} \frac{\partial \varphi}{\partial z}
$$

Based on the surface similarity theory (Monin and Obukhov 1954), $K_{\varphi}$ is then calculated as:

$$
K_{\varphi}=\frac{\kappa z u_{*}}{\phi_{\varphi_{M O S T}}} .
$$

where $\kappa$ is the von Kármán constant, represents the height above the displacement height, $u_{*}$ is the local friction velocity and $\phi_{\varphi_{M O S T}}$ is dimensionless stability function (Högström 1988). It is important to note that this is the most common way to parameterize (model) the surface-layer turbulence in meso- and large-scale models (e.g. WRF, IFS). One of the main drawbacks when applying the K-theory however is related to parameterization of physical and chemical processes describing the complex flow within and just above canopies and their oversimplification. In this thesis study, we investigated how high vegetation influences the exchange of energy, momentum and reactive compounds. Thus, following Harman and Finnigan $(2007 ; 2008)$, we used a modified MOST formulation for $K_{\varphi}$ (Eq. 5.3) by adding an additional function, $\hat{\phi}_{\varphi_{R S L}}$, to account for the canopy effects in the flux-gradient relationships (see Chapter 2 and 4 ):

$$
K_{\varphi}=\frac{\kappa z u_{*}}{\phi_{\varphi_{M O S T}} \hat{\phi}_{\varphi_{R S L}}} .
$$

Since we mainly focused our investigation on convective boundary layers (CBL), the use of the mixed-layer theory (e.g., Lilly 1968; Deardorff 1979) is applied to simplify Eq. (5.1) (see Chapters 3 and 4), while still retaining the main physical properties of the CBL.

Next to modelling, our method also used intensive and detailed measurements of micrometeorology and chemistry gathered during the Canopy Horizontal Array Turbulence Study (CHATS, Patton et al. 2011). The measurement campaign, and therefore the analysis, was done under leafless and leafy conditions. Our findings showed that the exchange of momentum, heat and moisture quantities within the roughness sublayer (quantified through $K_{\varphi}$ ) strongly depends on canopy sparsity/density and atmospheric stratification (Chapter 2). Since $K_{\varphi}$ is commonly used to parameterize the surface fluxes (for instance Eq. 5.2 with Eq. 5.3), and the latter are the lower boundary conditions in atmospheric models, it is important to investigate how the differences 
in $K_{\varphi}$, based on sparsity/density, affects the modelled surface fluxes and consequently the CBL (thermo)dynamics (Eq. 5.1). We further discuss these effects in Sections 5.1 and 5.2.

Furthermore, since the most relevant sources and sinks (III in Eq. 5.1) of chemical compounds are around the canopy, in Chapter 4 we focused our attention on quantifying the relevance of the chemistry in representing the budget of reactants (focusing on $\mathrm{O}_{3}, \mathrm{NO}, \mathrm{NO}_{2}$ ) over the CHATS canopy site. In Section 5.3, we further elaborate on how the chemical transformations influence the fluxes and source/sink distribution in Eq. (5.1). We extended the study by investigating the coupling between the surface fluxes, affected by high canopy, and the boundary-layer dynamics including large-scale effects, like mean vertical subsidence motions and/or the horizontal advection of heat and moisture (e.g. IV in Eq. 5.1). In doing so, we were able to obtain a complete interpretation of the diurnal variability of surface fluxes, boundary-layer height and the boundarylayer state variables in an integrated manner for the proposed case studies (e.g. Chapter 3). In the following, we focus on discussing these finding in relation to the concepts and processes that are still not well understood, and were not, or only partially treated in this study, but may have an impact on our findings. The main points treated here are related to:

1) the role of canopy sparsity and its impact on exchange fluxes and the boundary-layer dynamics;

2) the impact of atmospheric stability on exchange fluxes;

3) the influence of turbulence on mixing efficiency of chemically active species, and

4) the interaction between the surface processes, affected by canopy, and larger-scale processes such as advection and/or subsidence within the CBL system.

We close the chapter by providing an outlook on possible research paths to continue the current investigation.

\subsection{From dense to sparse canopies}

The atmospheric flows over the land surface in absence of canopy follow the rough-wall boundary layer. Under the presence of dense canopy (all the momentum absorbed by the upper part of the canopy), the flow within and just above the canopy is influenced by the roughness elements and behaves as a perturbed mixed layer (Raupach et al. 1996). Under these conditions the atmospheric flow is characterized by a strong inflection point of the mean wind velocity profile near the canopy top (Finnigan et al. 2009; Patton and Finnigan 2013) (see also Fig. 1.2 in Chapter 1). This perturbation however depends strongly on the density of the roughness elements. Dense canopies can be roughly seen as one large roughness element, whereas less dense or sparse canopies act as a porous media. In the following, we will elaborate how sparsity can be included in the roughnesssublayer parameterization used in Chapter 3 and 4 . 
By performing a flume experiment, Poggi et al. (2004) investigated the effects of intermediate (between rough-wall boundary layer and perturbed mixed layer) canopy density on the flow within the roughness sublayer. In their study, Poggi et al. (2004) provided a simple model formulation to account for the disturbed turbulent mixing in the vicinity of the canopy. Their model was based on the length scale related to the vortex size at the canopy-atmosphere interface $\left(L_{s}=U /[d U / d z]\right)$ (Raupach et al. 1996). This length scale however was never tested and evaluated on a real canopy study. Extending on the Poggi et al. (2004) study, Harman and Finnigan (2007; 2008) developed a model, based on their roughness-sublayer theory, to account for the canopy disturbed turbulence within and just above the canopy. The RSL theory developed by Harman and Finnigan $(2007 ; 2008)$ is here interpreted as a modification to the traditional similarity theory (Monin and Obukhov 1954) to account for the effects of the canopy on the flux-gradient relationships. Based on Harman and Finnigan's (2007; 2008) RSL theory, there are two additional scales that define the vortex size (thickness): the ratio between the friction velocity and the mean wind at canopy top, $\beta=u_{*} / U$, and the canopy adjustment length, $L_{c}$, which is a function of the drag coefficient and the one-sided plant area density $\left(L_{c}=\left[c_{d} a\right]^{-1}\right)$. These two scales define the displacement height and the roughness length for momentum and scalars (Harman and Finnigan 2007; 2008), which based on their model are stability dependent variables and not constants. The influence of canopy sparsity is introduced via the LAI, affecting the RSL scales $\beta$ and $L_{c}$ (see Table 5.1), which in turn affect the displacement height and the roughness length (Harman and Finnigan 2007; 2008).

Our approach and measurement data enable us to study the impact of canopy density/sparsity on turbulence. In Chapter 2, we consider a leafless canopy as a sparse canopy with a cumulative leaf area index LAI $=0.75$, whereas the canopy following the leaf-out represents a dense canopy with LAI = 2.5 (Table 5.1). By applying the RSL model (Harman and Finnigan 2007; 2008), and evaluating it against the CHATS dataset for the orchard canopy before and after leaf-out (Patton et al. 2011), we studied the changes of the turbulent mixing (via $K_{\varphi}$, see Eq. 5.3 in connection with Eq. 5.4) under leafless and leaf-out conditions for all measured atmospheric stability classes (see Chapter 2). To show the sensitivity of turbulence on canopy sparsity, we extend the study of Chapter 2 in this discussion chapter. The objective of this analysis is to investigate and show how high vegetation with different canopy density/sparsity affects the CBL dynamics. To answer this, we applied the MXL model with incorporated RSL parameterization when calculating the surface fluxes (see Chapter 3). We investigate the difference between leaf conditions by changing the LAI of the CHATS canopy before and after leaf-out. To represent the dense canopy, we use the already developed study case in Chapter 3 that focusing on canopy after leaf-out (LAI $=2.5$ ). To represent the sparse canopy, we use the information (measurements) for the LAI of the CHATS canopy before leaf-out (LAI=0.75) (see also Chapter 2). Other than this, the numerical run for the sparse canopy (or canopy before leaf-out) uses the same initial and boundary conditions as the numerical experiment for the dense canopy (or canopy after leaf-out). This kind of setting for the leafless canopy is of course an idealize case. Yet we use the idealized case to perform the 
Table 5.1: Differences in the numerical experiments set up for the canopy and roughness sublayer properties before and after leaf-out; $\beta$ and $L_{c}$ are calculated based on the CHATS measurements; the values for the vegetation fraction, $c_{\text {veg }}$, are taken in accordance to ECMWF IFS Documentation CY41RI (Part IV: Physical processes, Table 8.1).

\begin{tabular}{llll} 
& $\mathrm{LAI}\left[\mathrm{m}^{2} \mathrm{~m}^{-2}\right]$ & $\beta=u_{*} / U[-]$ & $L_{c}[\mathrm{~m}]$ \\
\hline Before leaf-out (sparse) & 0.75 & 0.2 & 52 \\
Aftar leaf-out (danse) & 2.5 & 0.3 & 16
\end{tabular}

numerical experiment to represent the sparse canopy due to the following two reasons:

i) because we are interested only in investigating the canopy density/sparsity effects on the CBL dynamics as represented through the RSL parameterization in the MXL model;

ii) because a new realistic case for canopy before leaf-out would bring additional uncertainty when comparing and discussing the results of the two case studies (leafless and canopy with leaves). This is due to the difference in the results between the two cases then being dependent on additional processes, such as radiative forcing, soil properties and large scale forcing.

Because of these reasons, we consider that the setup of the numerical experiment for leasless canopy based on an idealized case will suffice for our analysis. Table 5.1 summarizes the differences in the numerical set up for both experiments. The initial conditions of the numerical experiments are equal to the ones for the case study presented in Chapter 3 (see Appendix 3A).

Figure 5.1 shows the effect of canopy sparsity/density on the temporal variation of surface friction velocity and the mixed-layer mean wind velocity. In addition, the impact of canopy sparsity/density on the modelled temporal evolution of surface fluxes shown via the Bowen ratio, boundary-layer height, as well as the boundary state variables (e.g. potential temperature and specific humidity) is presented in Fig. 5.2 ( $a$ through $d$, respectively).

Figure 5.1a shows that the modelled friction velocity is lower for the case before leaf-out than after leaf-out. This is because the sparse canopy creates less shear compared to denser canopy (e.g. Raupach 1992). The larger differences between the values of the friction velocity in the beginning of the numerical runs, with tendency to equalise by the end of the run, can be explained with the fact that the friction velocity is calculated as a function of the mean wind and the drag coefficient for momentum. The lower friction velocity for the sparse canopy condition in the beginning of the experiment is due to a lower drag coefficient for momentum (see Chapter 3 ). The temporal variation in the mixed-layer mean wind speed between the sparse and the dense experiments (Fig. 5.1b) shows exactly the opposite trend: stronger wind speed over the sparse canopy increasing in time due to smaller drag). Consequently, the resulting friction velocity, calculated as a product of the wind speed and the square root of the drag coefficient of momentum (see Chapter 3), compensates the differences in the mean wind and the momentum drag. 

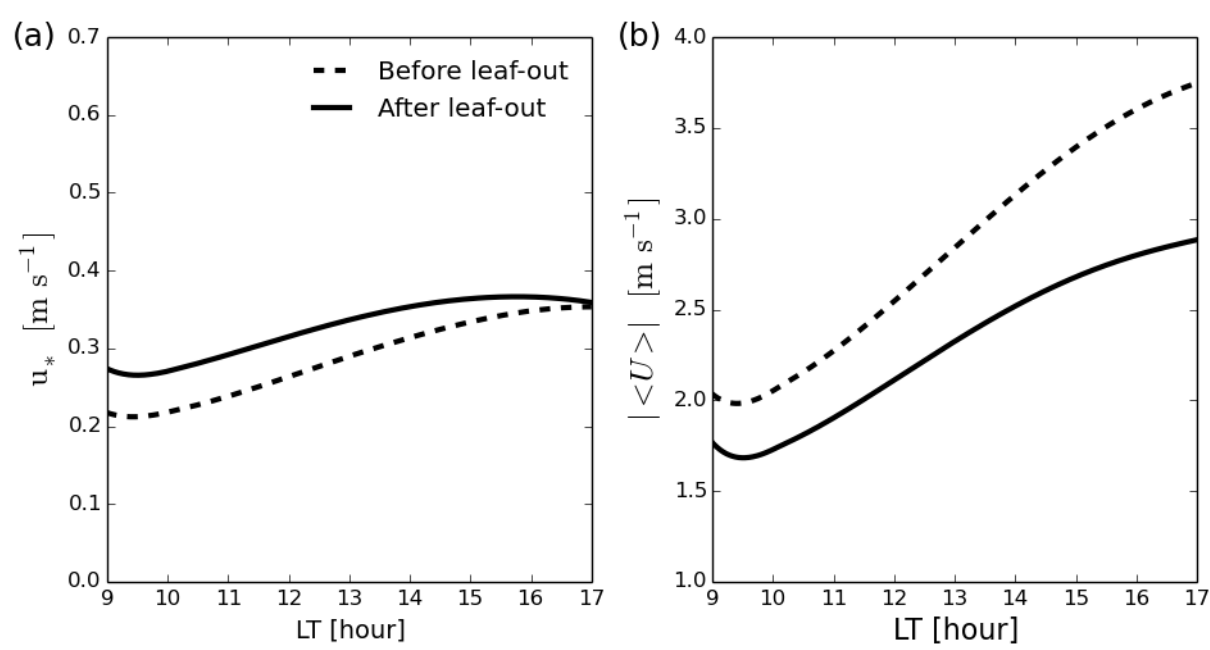

Figure 5.1: Diurnal evolution of (a) friction velocity and (b) the mixed-layer mean wind speed at $19 \mathrm{~m}$ above the canopy before $(L A I=0.7)$ and after leaf-out $(L A I=2.5)($ Table 5.1).

The changes from denser to sparser canopy significantly modifies the Bowen ratio, which in our case ranges from 0.25 to around unity by the end of the day (Fig. 5.2a). This is expected since the changes in vegetation fraction change the evapotranspirative exchange and strongly affect the latent heat flux. (e.g. Baldocchi et al. 2004; Kochendorfer and Ramírez 2008). The sparse canopy allows more solar insolation reaching the soil and thus generates stronger and faster sensible heat exchange (see Chapter 2) between the land surface and the atmosphere (Lawrence et al. 2007). This, of course, will depend on the soil properties and plant ecophysiology, which in our experiments remained equal. In this particular demonstration, the boundary layer grows deeper (Fig. 5.2b) due to changes in energy partitioning; the mixed-layer air is warmer and drier (Fig. 5.2c and 5.2d).

To summarize the results of the experiments, a sparser canopy yields increased mean wind, potential temperature, friction velocity and sensible heat partitioning, but decreases the specific humidity and the latent heat partitioning, thus increasing the boundary layer height. The analysis performed in this discussion, in which the canopy is treated as an integrated property of the CBL system, indicates that the vegetation density/sparsity has relevant impacts on the surface energy balance, and in consequence on the CBL dynamics. 

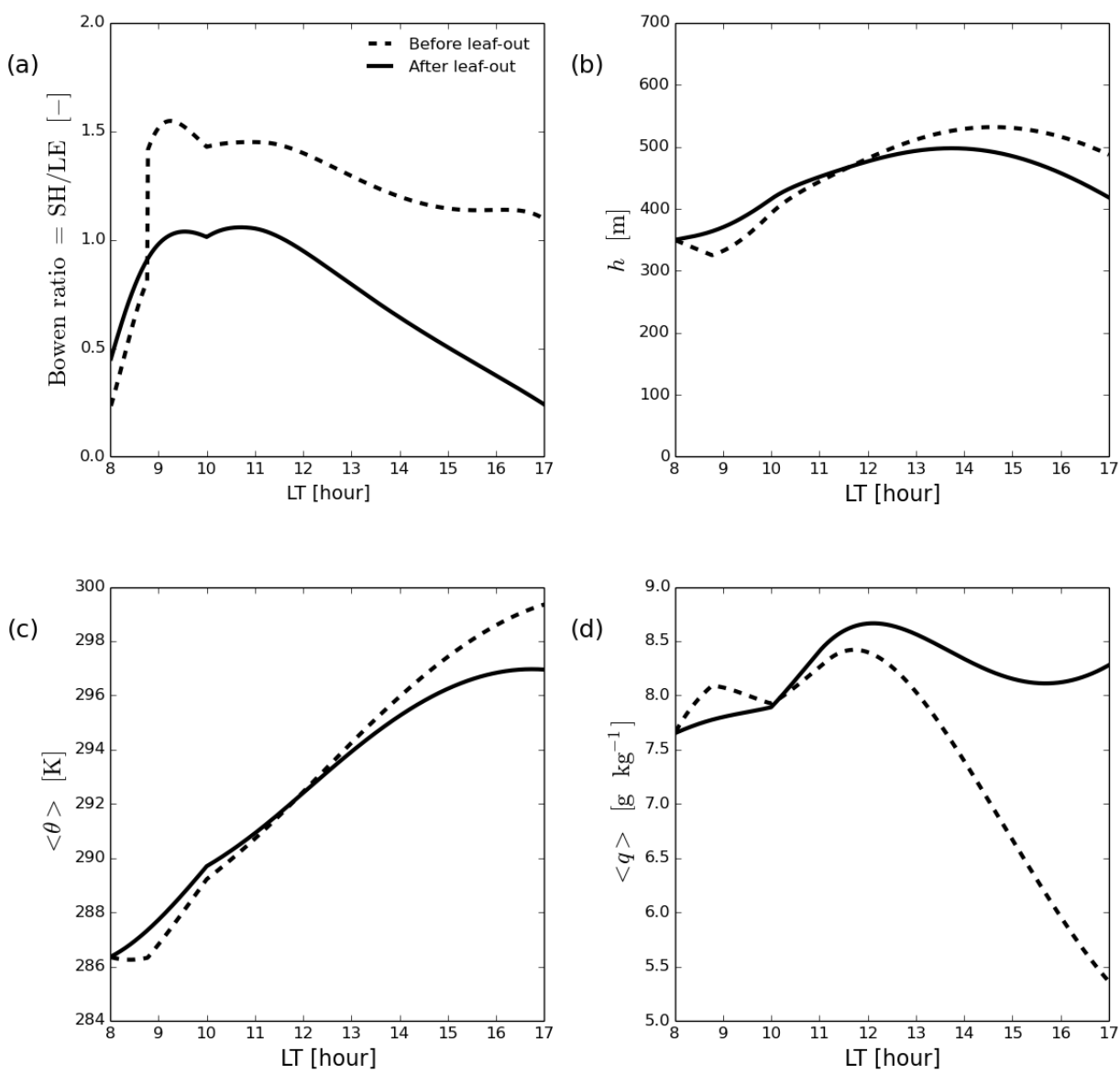

Figure 5.2: Diurnal evolution of (a) Bowen ratio, (b) boundary-layer height, (c) potential temperature and (d) specific humidity. Values in a,c, and $d$ are evaluated at $6 \mathrm{~m}$ above the canopy before $(L A I=0.75)$ and after leaf-out $(L A I=2.5)$ (Table 5.1). 


\subsection{From near-neutral conditions to increased atmospheric (in)stability}

Vertical fluxes within the roughness sublayer are dependent on the turbulent structure at the canopy-atmosphere interface (Gao et al. 1989; Raupach et al. 1996; Finnigan et al. 2009). As already mentioned, this shear driven turbulence is parameterized by using the thickness of the vortices cantered at the canopy top as a relevant scale within the RSL under near-neutral conditions (Harman and Finnigan 2007). In Chapter 2 by applying the RSL theory, we showed that it improves the turbulence parametrization for different canopy sparsity/density under near-neutral and weakly-unstable and weakly stable conditions. By using large eddy simulations, it was recently shown however that the turbulent structures within the RSL are strongly influenced by the larger atmospheric boundary-layer motions under free convection (Patton et al. 2016). This means that the effective exchange fluxes (II in Eq. 5.1) are influenced by these convective motions (Zilitinkevich et al. 2006), and have an effect on the entire boundary-layer system (Eq. 5.1). Therefore, there is a need of an additional turbulent scale in the RSL parameterization (next to $\beta$ and $L_{c}$ see Sect. 5.1) to account for the atmospheric boundary-layer motion (buoyancy driven) effects. It is important to note that while we mainly focus our thesis study on convective conditions, the turbulent structure within the RSL under stable conditions is also affected by the intermittent turbulence (e.g., Hollinger and Richardson 2005; Acevedo et al. 2006; Van Gorsel et al. 2011; Oliveira et al. 2013; Santos et al. 2016).

The existing RSL theory (parameterization) does not account for the boundary-layer scale motions (related to entrainment of warm and dry air) and intermittent transport that influences the turbulent exchange in the RSL, since these processes are still not well understood. A promising theoretical framework developed by Zilitinkevich et al. (2006) to account for the influence of large-scale convective motions on surface layer turbulence can be potentially extended to the roughness sublayer by incorporating and modifying the existing RSL theory (Harman and Finnigan 2007; 2008). In order to account for the intermittent transport under stable/nocturnal conditions, stochastic approaches are likely more appropriate (e.g. Monahan et al. 2015; Vercauteren and Klein 2015), since the nature of this intermittency is usually random and - more importantly - the mechanism not fully understood, and therefore difficult to capture with existing parameterizations.

\subsection{From passive to active chemical transformation in and above canopies}

The biosphere-atmosphere exchange of reactive compounds plays an important role in air quality and climate change by influencing global biogeochemical cycles, regulating the atmosphere's oxidation capacity, and moderating ozone and aerosol production (Fehsenfeld et al. 1992). The main mechanisms in this exchange include interactions between biogenic emissions, deposition, chemistry and turbulent transport (Seinfeld and Pandis 2016). Tall canopies are sources of reactive 


\subsection{FROM PASSIVE TO ACTIVE CHEMICAL TRANSFORMATION IN AND ABOVE CANOPIES}

carbon $\left(\mathrm{C}_{r}\right)$ and reactive nitrogen $\left(\mathrm{N}_{r}\right)$ compounds which control the oxidizing capacity, reflected by concentrations of the atmosphere's main 'detergent', the hydroxyl radical $(\mathrm{OH})$, and result in the production (or destruction) of ozone $\left(\mathrm{O}_{3}\right)$ and biogenic aerosols (Fehsenfeld et al. 1992). The effective emissions (i.e. the fraction of the primary emissions that leaves the canopy into the atmospheric boundary layer) of $\mathrm{C}_{r}$ and $\mathrm{N}_{r}$ compounds differ depending on their chemical lifetimes and the turbulent transport timescale (e.g. Foken et al. 2012).

Above vegetation, $\mathrm{C}_{r}$ enters the atmosphere in form of biogenic volatile organic compounds (BVOC) (Fuentes et al. 2000), such as isoprene and monoterpenes and higher terpenes as a function of plant species, sunlight and temperature (Guenther et al. 2000). These BVOCs are oxidized by $\mathrm{OH}$ but also by $\mathrm{O}_{3}$ and $\mathrm{NO}_{3}$. Consequently, increases in BVOC emissions, e.g., due to climate change, would generally result in a reduction of the oxidative capacity of the atmosphere, but this also depends on the pathway of the BVOCs oxidation (Lelieveld et al. 2008).

Soil microbial processes are major sources of natural $\mathrm{N}_{r}$, resulting in the emissions of nitric oxide (NO) (Guenther et al. 2000; Ganzeveld and Lelieveld 2004). The emitted NO reacts faster with $\mathrm{O}_{3}$ (characteristic time scale is minutes) resulting in the production of nitrogen dioxide $\left(\mathrm{NO}_{2}\right)$. The latter is removed by dry deposition resulting in an effective release of $\mathrm{NO}_{x}(\mathrm{NO}+$ $\mathrm{NO}_{2}$ ) into the atmosphere that can be less than $50 \%$ of the NO emitted by the soil (e.g. Ganzeveld et al. 2002). This is of particular relevance because NOx acts as a key catalyst in the formation of $\mathrm{O}_{3}$. In addition, $\mathrm{NO}_{x}$ is involved in the production of $\mathrm{OH}$, demonstrating the intrinsic links that exist between the atmosphere-biosphere exchanges of $\mathrm{N}_{r}, \mathrm{C}_{r}$, and $\mathrm{O}_{3}$.

Another important process related to canopy-atmosphere exchange of $\mathrm{N}_{r}, \mathrm{C}_{r}$, and $\mathrm{O}_{3}$ is the removal of trace gases and aerosols by the vegetation and soil surfaces through dry deposition. Trace gases are transported to the surfaces of leafs, trunks and soils by turbulence and are deposited onto these surfaces or taken up by leaf stomata (Seinfeld and Pandis 2016). The biological controls that influence the uptake of compounds are closely linked to environmental conditions and include internal $\mathrm{CO}_{2}$ concentration, and leaf temperature (Seinfeld and Pandis 2016).

An important feature of this combined $\mathrm{N}_{r}, \mathrm{C}_{r}$, and $\mathrm{O}_{3}$ canopy-atmosphere exchange is that a number of these chemical interactions and processes occur on timescales $\left(t_{c}\right)$ that are comparable to the turbulent transport timescale $\left(t_{t}\right)$ implying that the effective exchange, as well as canopy interaction strongly depend on turbulent transport (Patton et al. 2001).

The processes described above suggest the necessity to have an adequate description of the effective fluxes of reactive compounds within and above the canopy. Therefore, in Chapter 4 we discussed the importance of including the canopy effects when calculating the deposition/emission fluxes of reactive species from their measured concentration profiles within and above the canopy. To calculate the vertical fluxes of reactive compounds and to account for the canopy RSL effects, we use Eq. 5.2 and Eq. 5.4.

Although we show improvement in the calculation of the emission/deposition fluxes of reactants within the RSL by comparing with the CHATS data, we also found that the reactant flux 
calculated using the mean gradients of the reactive species strongly vary with height, i.e flux divergence departing from zero (see e.g. Fig. 4.12 in Chapter 4). The reason for this flux divergence is most likely related to the chemical transformation of reactants. In short, the formation and depletion of species due to reactions create an extra source or sink that modifies the flux between two points at different heights (between two levels). Several experimental (Duyzer et al. 1983; Delany et al. 1986; Wesely et al. 1989) and modelling studies (Fitzjarrald and Lenschow 1983; Gao et al. 1991; Vilà-Guerau de Arellano and Duynkerke 1992) showed that the flux-gradient relationships based on Eq. 5.2 with Eq. 5.3 are influenced by the chemical transformation of the reactants. Thus, when calculating vertical fluxes of reactants within the RSL the use of an additional function $\phi_{\text {chem }}$ is recommended to account for the local sources/sinks due to chemical transformation of reactants (Vilà-Guerau de Arellano et al. 1995):

$$
K_{\varphi}=\frac{\kappa z u_{*}}{\phi_{\varphi_{M O S T}} \hat{\phi}_{\varphi_{R S L}} \phi_{\text {chem }}} .
$$

where $\phi_{\text {chem }}$ is a function of the ratios of the turbulent and chemistry time scales of the investigated reactants, as well as the ratios of the vertical fluxes of those reactants (Vilà-Guerau de Arellano et al. 1995). Consequently, the improved definition of the exchange coefficient within the RSL should take not only the effects of the atmospheric stability and the tall canopy into account, but also the effects of the chemical transformation of species to more precisely connect the gradient of those reactants to the corresponding effective fluxes. The chemical transformation of reactants affect the surface effective fluxes of these reactants, needed as lower boundaries of an atmospheric chemistry model (e.g. the second terms in Eq. 5.1) (Vilà-Guerau de Arellano et al. 1995).

Chemistry can also have an effect on the total budget of these reactants in the convective boundary layer via the source/sink term in Eq. 5.1 due to incomplete mixing (e.g. Schumann 1989; Vilà-Guerau de Arellano and Duynkerke 1993; Gao and Wesely 1994; Sykes et al. 1994; Krol et al. 2000; Ouwersloot et al. 2011). The statistical variable that describes the effect of these incomplete mixing effects on chemistry is called intensity of segregation $\left(I_{s}\right)$ (Danckwerts 1952) defined as:

$$
I_{s}=\frac{\left[c_{A}^{\prime} c_{B}^{\prime}\right]}{\left[c_{A}\right]\left[c_{B}\right]},
$$

where $c_{A}$ and $c_{B}$ represent the mixing ratios of reactive compounds $\mathrm{A}$ and $\mathrm{B}$ respectively; the rectangular brackets correspond to an average and the prime corresponds to a deviation from the spatial average. The effects of the segregation is largest when the time scale of the turbulent mixing is comparable with the time scale of the chemical reactions (e.g. Damköhler 1957). Within the RSL the transport and chemistry are influenced by non-linear mixing processes which cause inhomogenities in scalar fields (e.g. Raupach et al. 1996; Katul et al. 1997; Patton et al. 2001), which potentially influence the segregation of reactants. A number of experimental and modelling studies investigated the segregation (e.g. Gao et al. 1993; Makar et al. 1999; Patton et al. 
2001; Karl et al. 2007; Butler et al. 2008; Dlugi et al. 2010) within and above high vegetation (forests). They all reported the importance of the intensity of segregation when studying and representing the atmospheric reactive constituents in the CBL over high vegetation. Next to the detailed turbulence observations, it is important for future measurement experiments of reactive compounds, to include observations of the mean profiles of reactant concentrations together with variances of their mixing ratios and their vertical fluxes. This allows the evaluation of the intensity of segregation (Eq. 5.6) and this term can be added to the total budget of the reactants under investigation.

\subsection{From isolated canopy to canopy coupled to larger-scale processes}

One of the most innovative aspects of this study is the approach aiming to integrate the tall vegetation within the atmospheric boundary-layer system to represent the CBL dynamics and the chemistry over an orchard canopy. In the previous two sections, we reported the influence of the canopy sparsity/density on the parameterization of surface fluxes, reactants, and CBL dynamics. Other processes, with non-local characteristics, that can influence the exchange of momentum, energy, and reactive compounds between the canopies and the atmosphere are related to large(meso) scale forcings: advection and/or subsidence. Advection affects the ABL dynamics and the boundary-layer state variables (see Chapter 3) directly as air masses with different properties replaces the air of the investigated domain (the last term in Eq. 5.1). Subsidence influences the CBL system (Eq. 5.1) and the boundary-layer growth as large-scale vertical motions, driven by the surface topography (return flow motions), can form a stable (high pressure) field above the mixed layer that usually suppresses the growth of the boundary layer. These large-scale advective and subsidence processes, driven by the complex topography of the California valley, influence the CHATS experimental site (Zaremba and Carroll 1999b; Bianco et al. 2011; Mayor 2011). These large-scale processes affect the diurnal variability of the state variables above the canopy (Chapter 3 and 4). By using the mixed-layer model, we account for these large-scale processes by imposing constant values in time for the advection and subsidence driven by the observation of the boundary-layer height and mixed-layer thermodynamic variables and atmospheric constituents. As a first guess, this approach was sufficient to study the effects of high vegetation on exchange processes between the canopy and the atmosphere. Other methods should be used, however, when a more detailed analysis of the combined effects of canopy and large-scale processes on the CBL dynamics is required. For example, analysis of the regional climatology from mesoscale models (e.g. WRF, WRF-CHEM) with a RSL parameterization incorporated (e.g. Harman and Finnigan 2007; 2008) can provide information about sea breeze and recirculation processes due to topography (e.g. Bianco et al. 2011) and their interaction with high vegetation. In addition, direct radio-sounding measurements throughout the entire boundary layer can also provide important information about these large-scale processes. These measurements can 
also help in more precise initialization and validation of numerical experiments that aim to constrain the budgets of atmospheric quantities. This is important since the main uncertainty in this study, when studying the budgets of boundary-state variables and reactive compounds, is related to the lack of knowledge on the initial and boundary conditions in the entrainment zone. For those reasons, additional measurement campaigns are needed in which detailed observations of the turbulence and the chemistry within the entire ABL system should be conducted. Next to that, enabled by increasing computation power, new numerical experiments using LES can support the measurements and further improve our understanding about the exchange processes of energy, momentum and passive and active chemical compounds and their role in the climate system.

In closing, it is important to stress that there are current initiatives on experiments and model studies that aim to investigate (i) the role of sparsity/density on intermittent transport, (ii) chemistry and turbulence interaction and (iii) large-scale processes on CBL dynamics and chemistry. In that respect it is worth to mention the ongoing GRAPEX project (Grape Remote sensing Atmosphere Profiling and Evapotranspiration eXchange) in which turbulent transport processes in vineyards (as sparse canopies) and their effect on evapotranspiration are studied (Kustas et al. 2016). Specific focus in this project is placed on studying the intermittent turbulent transport. The ATTO (Amazonian Tall Tower Observatory) campaign is another relevant project aiming at delivering ground-breaking findings which will be the basis for improved climate models. Furthermore, applying high resolution Large Eddy Simulations (LES) on canopy-atmosphere interaction is becoming more widespread. Recent LES studies include coupled chemical transformation schemes (Li et al. 2016) to study exchange of reactive compounds between canopies and atmosphere. Finally, studying the tall canopy effects in an ABL system and taking the large-scale processes into account (as presented in this study) is crucial to improve the understanding of exchange processes between the canopy and the atmosphere. In this line of thought, the RSL theory (Harman and Finnigan 2007; 2008) (e.g. Eq. 5.4) is currently being implemented in the Community Land Model (CLM) (e.g. Bonan and Levis 2006) (Dr. Edward (Ned) Patton personal communication), coordinated and developed by the National Center for Atmospheric Research (NCAR). 


\section{6}

\section{Summary}

This chapter summarizes the most relevant findings of this $P h D$ research project. As the results are presented and discussed in detail in Chapters 2 to 5, only the most important conclusions are presented here.

The systematic investigation in this thesis showed the relevance of the roughness sublayer (RSL) for the turbulent exchange processes between the atmosphere and the land surface characterized by high vegetation. More specifically, we showed and quantified the effects of the disrupted flow due to canopy on the parameterization of the vertical turbulent fluxes of momentum, heat, moisture and reactive gases within and above the canopy. The originality of the research lies in the using real-world case studies and the connection between atmospheric scales, in addition to the canopy scale. To this end, we elaborated how, and to what extent, the modelled surface fluxes, affected by the presence of high vegetation with different canopy phenology, impact the diurnal evolution of the convective boundary-layer (thermo-)dynamics and its reactive chemical composition. In what follows, we briefly summarized the main findings in this thesis, in connection with the research questions posed at the introduction:

- Question 1: What are the effects of canopy phenology and atmospheric stability on turbulent exchange of energy and momentum within the roughness sublayer?

By extensively analysing the complete data set above the orchard canopy (over a growing season) from the Canopy Horizontal Array Turbulence Study (CHATS) experiment, we explained 
and discussed in Chapter 2 how the turbulence parameterization within the roughness sublayer is strongly dependent on canopy-phenology (canopy leaf state) and atmospheric-stability changes, from unstable to stable conditions. More specifically, the turbulent exchange of momentum and energy in the roughness sublayer, as quantified by the turbulent exchange coefficient, is largely affected by the canopy leaf state. Our analysis showed that the turbulent mixing is more intense under leafless than under full-leaf canopy, especially during unstable and near-neutral conditions. This is due to the most efficient atmosphere-canopy coupling under leafless canopy conditions. This was further demonstrated by the analysis on the vorticity thickness (defined as the thickness of the turbulent eddies created at canopy vicinity) - a relevant turbulent length scale in the roughness sublayer (see Chapter 2). Our findings indicate that using appropriate turbulent (mixing) length scales in the roughness sublayer (vorticity thickness, displacement height), taking the canopy phenology and atmospheric stability changes into account, is crucial to accurately calculate fluxes from observed mean gradients when applying the flux-gradient relationships in the roughness sublayer. We further showed that applying such turbulent and canopy scales in the roughness sublayer parameterization for the canopy-induced modification (Harman and Finnigan 2007; 2008) improves the calculated flux-gradient relationships and thus the turbulent exchange coefficients, as corroborated with the CHATS observations.

- Question 2: What is the impact of the roughness sublayer in representing the dynamics of the convective boundary layer?

In Chapter 3, we studied the roughness sublayer in broader perspective by integrating it as a part of the land-convective boundary-layer system. We did so by integrating a state-of theart roughness sublayer parameterization (as already introduced to answer Question 1) within the surface scheme of a soil-vegetation-atmosphere mixed-layer model. We selected and represented two cases with characteristic wind forcing over the CHATS canopy (one case with southerly dominant winds and another case with changing wind direction from northerly to southerly) to ensure canopy fetch with well-developed roughness sublayer. The model initialization and boundary conditions were supported by the CHATS observations. Due to the complexity of the CHATS site, we also considered external large-scale processes such as advection of heat and moisture, as well as subsidence into the system. Our analysis showed that the CHATS boundary-layer dynamics are largely affected and controlled by the large-scale processes (advection and subsidence) related to the coastal see breeze and the regional topography. The effect of the canopy and the roughness sublayer on the CHATS boundary layer dynamics for the investigated case studies was relatively small (e.g. up to few percent roughness sublayer effect on the boundary layer growth). This is due to small roughness sublayer effects on surface fluxes. Since in our modelling framework (and normally in land-atmosphere models) all fluxes are forced by the boundary conditions (sensible and latent heat fluxes are bounded by the available energy, momentum is bounded by the pressure gradient and the boundary-layer growth), they do not change much if the way of 
modelling is changed, but rather their partitioning is changed. In that respect, and specifically for our case studies, we found that including or omitting the roughness-sublayer effects in the parameterization of the modelled surface fluxes alters the partitioning between the sensible, the latent and the ground heat fluxes with only few percent. These small variations in the surface fluxes are related to the way the roughness length and displacement height are used in the parameterization; the roughness sublayer parameterization determines the roughness length and displacement height as stability depended variables, contrary to the standard similarity theory based on which these are fixed parameters.

Near the canopy top however, the orchard canopy had a significant impact on the boundary layer state variables (wind speed, potential temperature and specific humidity) and the corresponding turbulent transfer coefficients (drag coefficients for momentum and scalars) near the canopy top. This finding was supported by the CHATS observations at the canopy height.

Moreover, the sensitivity analysis on canopy and roughness sublayer scales indicated that the roughness-sublayer effects on the convective boundary-layer dynamics might be significantly larger for taller and denser canopies compared to the $10 \mathrm{~m}$ tall orchard canopy in our study. In that respect, additional real case studies, including variety of canopy types with different characteristics (height and densities), need to be conducted to further support and generalize the conclusions of this study.

- Question 3: What is the contribution of physical and chemical processes to the ozone budget above the CHATS canopy, specifically focusing on ozone surface deposition?

The case study of the boundary-layer thermodynamics with southerly dominant winds, studied in detail in Chapter 3, served as a base to study the roughness-sublayer effects on the ozone budget over the CHATS canopy in Chapter 4. The roughness sublayer effects on ozone budget (next to the effects of entrainment, chemistry, advection) are studied via the ozone dry deposition since the latter is directly influenced by the presence of high vegetation. Thus, we first quantified the roughness-sublayer effects on retrieved deposition fluxes of ozone (and other reactants) within and above the canopy. We applied and compared different flux-retrieval methods on the observed mixing ratios above the CHATS canopy to account for or omit the roughness sublayer effects. We diagnosed twice-larger magnitude of the deposition fluxes when the roughness sublayer effects are taken into account in the flux-gradient relationship, compared to the method which neglects these effects. When applied in the mixed-layer model, this underestimation of the deposition fluxes, as parameterized via the ozone deposition velocity and the ozone mixing ratios, led to a relevant overestimation of the ozone diurnal mixing ratio (16\% in the daily average), as represented by the model.

The CHATS chemistry in this study is complex and covers low ozone and high $\mathrm{NO}_{x}$ regime. The altered photostationary state, as quantified by the high Leighton ratio (around 3), indicated that additional reactions than the triad $\mathrm{NO}_{2}-\mathrm{NO}-\mathrm{O}_{3}$ scheme convert $\mathrm{NO}_{x}$ to $\mathrm{O}_{3}$ via $\mathrm{PO}_{2}$. Most 
likely, these pathways involve natural hydrocarbons (volatile organic compounds-VOCs) emitted by the canopy. As indicated by the ozone budget analysis for the investigated case, in case of increased VOCs emissions, an outflux of ozone was likely present to ensure low ozone mixing ratio over CHATS. Although the mixed-layer model again showed to be powerful tool when studying convective boundary-layer dynamics and chemistry, for more detailed representation of the ozone- $\mathrm{NO}_{x}$-VOCs chemistry above the CHATS canopy more detailed observations would be needed. 


\section{References}

Acevedo, O., OL, M., Degrazia, G., and Medeiros, L.: Intermittency and the exchange of scalars in the nocturnal surface layer, Boundary-layer Meteorology, 119, 41-55, 2006.

Altshuler, S., Arcado, T., and Lawson, D.: Weekday vs. weekend ambient ozone concentrations: discussion and hypotheses with focus on northern California, Journal of the Air and Waste Management Association, 45, 967-972, 1995.

Anderson, M., Norman, J., Diak, G., Kustas, W., and Mecikalski, J.: A two-source time-integrated model for estimating surface fluxes using thermal infrared remote sensing, Remote Sensing of Environment, 60, 195-216, 1997.

Aubinet, M., Grelle, A., Ibrom, A., Rannik, U., Moncrieff, J., Foken, T., Kowalski, A., Martin, P., Berbigier, P., and Bernhofer, C.: Estimates of the annual net carbon and water exchange of forests: the EUROFLUX methodology, Advances in Ecological Research, 30, 113-175, 1999.

Baldocchi, D. and Harley, P.: Scaling carbon dioxide and water vapour exchange from leaf to canopy in a deciduous forest. II. Model testing and application, Plant, Cell and Environment, 18, 1157-1173, 1995.

Baldocchi, D., Falge, E., Gu, L., Olson, R., Hollinger, D., Running, S., Anthoni, P., Bernhofer, C., Davis, K., Evans, R., Fuentes, J., Goldstein, A., Katul, G., Law, B., Lee, X., Malhi, Y., Meyers, T., Munger, W., Oechel, W., Paw, K. T., Pilegaard, K., Schmid, H. P., Valentini, R., Verma, S., 
Vesala, T., Wilson, K., and Wofsy, S.: FLUXNET: A New Tool to Study the Temporal and Spatial Variability of EcosystemâĂŞScale Carbon Dioxide, Water Vapor, and Energy Flux Densities, Bulletin of the American Meteorological Society, 82, 2415-2434, doi:doi:10.1175/15200477(2001)082<2415:FANTTS>2.3.CO;2, 2001.

Baldocchi, D., Xu, L., and Kiang, N.: How plant functional-type, weather, seasonal drought, and soil physical properties alter water and energy fluxes of an oakâĂŞgrass savanna and an annual grassland, Agricultural and Forest Meteorology, 123, 13-39, doi:http://dx.doi.org/10.1016/j.agrformet.2003.11.006, 2004.

Bao, J., Michelson, S., Persson, P., Djalalova, I., and Wilczak, J.: Observed and WRF-simulated low-level winds in a high-ozone episode during the Central California Ozone Study, Journal of Applied Meteorology and Climatology, 47, 2372-2394, 2008.

Belcher, S., Jerram, N., and Hunt, J.: Adjustment of a turbulent boundary layer to a canopy of roughness elements, Journal of Fluid Mechanics, 488, 369-398, doi:doi:10.1017/S0022112003005019, 2003.

Betts, A.: A composite mesoscale cumulonimbus budget, Journal of the Atmospheric Sciences, 30, 597-610, 1973a.

Betts, A.: NonâĂŘprecipitating cumulus convection and its parameterization, Quarterly Journal of the Royal Meteorological Society, 99, 178-196, 1973b.

Betts, A., Ball, J., Beljaars, A., Miller, M., and Viterbo, P.: The land surface-atmosphere interaction: A review based on observational and global modeling perspectives, Journal of Geophysical Research: Atmospheres, 101, 7209-7225, 1996.

Bianco, L., Djalalova, I., King, C., and Wilczak, J.: Diurnal Evolution and Annual Variability of Boundary-Layer Height and Its Correlation to Other Meteorological Variables in CaliforniaâĂŹs Central Valley, Boundary-Layar Meteorology, 140, 491-511, doi:10.1007/s10546-0119622-4, 2011.

Bohrer, G., Katul, G. G., Walko, R. L., and Avissar, R.: Exploring the effects of microscale structural heterogeneity of forest canopies using large-eddy simulations, Boundary-Layer Meteorology, 132, 351-382, 2009.

Bonan, G.: Forests and climate change: forcings, feedbacks, and the climate benefits of forests, Science, 320, 1444-1449, 2008.

Bonan, G.: Ecological climatology: concepts and applications, Cambridge University Press, 2015. 
Bonan, G. and Levis, S.: Evaluating aspects of the community land and atmosphere models (CLM3 and CAM3) using a dynamic global vegetation model, Journal of Climate, 19, 22902301, 2006.

Boussetta, S., Balsamo, G., Beljaars, A., Panareda, A., Calvet, J., Jacobs, C., Hurk, B., Viterbo, P., Lafont, S., and Dutra, E.: Natural land carbon dioxide exchanges in the ECMWF Integrated Forecasting System: Implementation and offline validation, Journal of Geophysical Research: Atmospheres, 118, 5923-5946, 2013.

Boy, M., Sogachev, A., Lauros, J., Zhou, L., Guenther, A., and Smolander, S.: SOSA - A new model to simulate the concentrations of organic vapours and sulphuric acid inside the ABL-Part 1: Model description and initial evaluation, Atmospheric Chemistry and Physics, 11, 43-51, 2011 .

Brunet, Y., Finnigan, J., and Raupach, M.: A wind tunnel study of air flow in waving wheat: Single-point velocity statistics, Boundary-Layerer Meteorology, 70, 95-132, doi:10.1007/bf00712525, 1994.

Businger, J., Wyngaard, J., Izumi, Y., and Bradley, E.: Flux-Profile Relationships in the Atmospheric Surface Layer, Journal of the Atmospheric Sciences, 28, 181-189, doi:doi:10.1175/1520-0469(1971)028<0181:FPRITA>2.0.CO;2, 1971.

Butler, T., Taraborrelli, D., Brühl, C., Fischer, H., Harder, H., Martinez, M., Williams, J., Lawrence, M., and Lelieveld, J.: Improved simulation of isoprene oxidation chemistry with the ECHAM5/MESSy chemistry-climate model: lessons from the GABRIEL airborne field campaign, Atmospheric Chemistry and Physics, 8, 4529-4546, 2008.

Campbell, G. and Norman, J.: Introduction to environmental biophysics, Springer Verlag, 1998.

Carpenter, L., Clemitshaw, K., Burgess, R., Penkett, S., Cape, J., and McFadyen, G.: Investigation and evaluation of the NO x/O 3 photochemical steady state, Atmospheric Environment, 32, 3353-3365, 1998.

Carson, D.: The development of a dry inversionâĂ ̌̌capped convectively unstable boundary layer, Quarterly Journal of the Royal Meteorological Society, 99, 450-467, 1973.

Casso-Torralba, P., Vilà-Guerau de Arellano, J., Bosveld, F., Soler, M., Vermeulen, A., Werner, C., and Moors, E.: Diurnal and vertical variability of the sensible heat and carbon dioxide budgets in the atmospheric surface layer, Journal of Geophysical Research: Atmospheres, 113, D12 119, doi:10.1029/2007jd009583, URL http: //dx. doi .org/10 . 1029/2007 JD009583, 2008 . 
Cellier, P. and Brunet, Y.: Flux-gradient relationships above tall plant canopies, Agricultural and Forest Meteorology, 58, 93-117, 1992.

Chen, F. and Dudhia, J.: Coupling an advanced land surface-hydrology model with the Penn State-NCAR MM5 modeling system. Part I: Model implementation and sensitivity, Monthly Weather Review, 129, 569-585, 2001.

Chen, F. and Schwerdtfeger, P.: Flux-gradient relationships for momentum and heat over a rough natural surface, Quarterly Journal of the Royal Meteorological Society, 115, 335-352, 1989.

Claussen, M., Cox, P., Zeng, X., Viterbo, P., Beljaars, A., Betts, R., Bolle, H., Chase, T., and Koster, R.: Vegetation, Water, Humans and the Climate: A New Perspective on an Interactive System, Global Change - The IGBP Series, Springer-Verlag Berlin Heidelberg, 1 edn., doi:10.1007/978-3-642-18948-7, 2004.

Conzemius, R. and Fedorovich, E.: Dynamics of Sheared Convective Boundary Layer Entrainment. Part II: Evaluation of Bulk Model Predictions of Entrainment Flux, Journal of the Atmospheric Sciences, 63, 1179-1199, doi:10.1175/jas3696.1, 2006.

Conzemius, R. and Fedorovich, E.: Bulk Models of the Sheared Convective Boundary Layer: Evaluation through Large Eddy Simulations, Journal of the Atmospheric Sciences, 64, 786807, doi:doi:10.1175/JAS3870.1, 2007.

Danckwerts, P.: The definition and measurement of some characteristics of mixtures, Applied Scientific Research, Section A, 3, 279-296, 1952.

De Ridder, K.: Bulk transfer relations for the roughness sublayer, Boundary-Layer Meteorology, 134, 257-267, 2010.

Deardorff, J.: Prediction of convective mixed-layer entrainment for realistic capping inversion structure, Journal of the Atmospheric Sciences, 36, 424-436, 1979.

Delany, A., Fitzjarrald, D., Lenschow, D., Pearson Jr, R., Wendel, G., and Woodrufl, B.: Direct measurements of nitrogen oxides and ozone fluxes over grassland, Journal of Atmospheric Chemistry, 4, 429-444, 1986.

DeMore, W. B., Sander, S. P., Golden, D., Hampson, R., Kurylo, M. J., Howard, C. J., Ravishankara, A., Kolb, C., and Molina, M.: Chemical kinetics and photochemical data for use in stratospheric modeling. evaluation no. 12, 1997.

Denmead, O. and Bradley, E.: Flux-Gradient Relationships in a Forest Canopy, book section 27, pp. 421-442, Springer Netherlands, doi:10.1007/978-94-009-5305-5 27, 1985. 
Denmead, O. and Bradley, E. F.: On scalar transport in plant canopies, Irrigation Science, 8, 131-149, 1987.

Dickinson, R.: Land surface processes and climate-surface albedos and energy balance, Advances in Geophysics, 25, 305-353, 1983.

Dickinson, R. and Henderson-Sellers, A.: Modelling tropical deforestation: A study of GCM land-surface parametrizations, Quarterly Journal of the Royal Meteorological Society, 114, 439-462, doi:10.1002/qj.49711448009, 1988.

Dillon, M., Lamanna, M., Schade, G., Goldstein, A., and Cohen, R.: Chemical evolution of the Sacramento urban plume: Transport and oxidation, Journal of Geophysical Research: Atmospheres, 107, 2002.

Dlugi, R., Berger, M., Zelger, M., Hofzumahaus, A., Siese, M., Holland, F., Wisthaler, A., Grabmer, W., Hansel, A., and Koppmann, R.: Turbulent exchange and segregation of $\mathrm{HO} x$ radicals and volatile organic compounds above a deciduous forest, Atmospheric Chemistry and Physics, 10, 6215-6235, 2010.

Dupont, S. and Patton, E.: Momentum and scalar transport within a vegetation canopy following atmospheric stability and seasonal canopy changes: The CHATS experiment, Atmospheric Chemistry and Physics, 12, 5913-5935, 2012a.

Dupont, S. and Patton, E.: Influence of stability and seasonal canopy changes on micrometeorology within and above an orchard canopy: The CHATS experiment, Agricultural and Forest Meteorology, 157, 11-29, doi:http://dx.doi.org/10.1016/j.agrformet.2012.01.011, 2012b.

Duyzer, J., Meyer, G., and Van Aalst, R.: Preliminary communication Measurement of dry deposition velocities of NO, N and $\mathrm{O} 3$ and the influence of chemical reactions, Atmospheric Environment (1967), 17, 2117-2120, 1983.

Dyer, A.: A review of flux-profile relationships, Boundary-Layer Meteorology, 7, 363-372, 1974.

Dyer, A., Garratt, J., Francey, R., McIlroy, I., Bacon, N., Hyson, P., Bradley, E. F., Denmead, O., Tsvang, L., and Volkov, Y.: An international turbulence comparison experiment (ITCE 1976), Boundary-Layer Meteorology, 24, 181-209, 1982.

Eder, F., Serafimovich, A., and Foken, T.: Coherent Structures at a Forest Edge: Properties, Coupling and Impact of Secondary Circulations, Boundary-Layer Meteorology, pp. 1-24, 2013.

Endlich, R., Ludwig, F., and Uthe, E.: An automatic method for determining the mixing depth from lidar observations, Atmospheric Environment, 13, 1051-1056, 1979. 
Fares, S., McKay, M., Holzinger, R., and Goldstein, A. H.: Ozone fluxes in a Pinus ponderosa ecosystem are dominated by non-stomatal processes: evidence from long-term continuous measurements, Agricultural and Forest Meteorology, 150, 420-431, 2010.

Fares, S., Weber, R., Park, J., Gentner, D., Karlik, J., and Goldstein, A. H.: Ozone deposition to an orange orchard: Partitioning between stomatal and Âănon-stomatal sinks, Environmental Pollution, 169, 258-266, doi:http://dx.doi.org/10.1016/j.envpol.2012.01.030, 2012.

Fares, S., Matteucci, G., Mugnozza, G., Morani, A., Calfapietra, C., Salvatori, E., Fusaro, L., Manes, F., and Loreto, F.: Testing of models of stomatal ozone fluxes with field measurements in a mixed Mediterranean forest, Atmospheric Environment, 67, 242-251, 2013.

Fares, S., Savi, F., Muller, J., Matteucci, G., and Paoletti, E.: Simultaneous measurements of above and below canopy ozone fluxes help partitioning ozone deposition between its various sinks in a Mediterranean Oak Forest, Agricultural and Forest Meteorology, 198, 181-191, 2014.

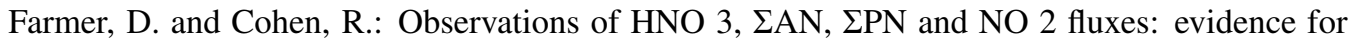
rapid HO x chemistry within a pine forest canopy, Atmospheric Chemistry and Physics, 8, 3899-3917, 2008.

Fedorovich, E.: Modeling the Atmospheric Convective Boundary Layer within a Zero-Order Jump Approach: An Extended Theoretical Framework, Journal of Applied Meteorology, 34, 1916-1928, doi:10.1175/1520-0450(1995)034<1916:mtacbl>2.0.co;2, 1995.

Fehsenfeld, F., Calvert, J., Fall, R., Goldan, P., Guenther, A., Hewitt, C., Lamb, B., Liu, S., Trainer, M., Westberg, H., and Zimmerman, P.: Emissions of volatile organic compounds from vegetation and the implications for atmospheric chemistry, Global Biogeochemical Cycles, 6, 389-430, doi:10.1029/92gb02125, 1992.

Finnigan, J.: Turbulence in plant canopies, Annual Review of Fluid Mechanics, 32, 519-571, 2000 .

Finnigan, J., Shaw, R., and Patton, E.: Turbulence structure above a vegetation canopy, J. Fluid Mech., 637, 387-424, 2009.

Fitzjarrald, D. and Lenschow, D.: Mean concentration and flux profiles for chemically reactive species in the atmospheric surface layer, Atmospheric Environment (1967), 17, 2505-2512, 1983.

Fitzjarrald, D., Stormwind, B., Fisch, G., and Cabral, O.: Turbulent transport observed just above the Amazon forest, Journal of Geophysical Research: Atmosphere, 93, 1551-1563, doi:10.1029/JD093iD02p01551, 1988. 
Fitzjarrald, D., Acevedo, O., and Moore, K.: Climatic consequences of leaf presence in the eastern United States, Journal of Climate, 14, 598-614, 2001.

Foken, T.: 50 Years of the Monin-Obukhov Similarity Theory, Boundary-Layer Meteorology, 119, 431-447, doi:10.1007/s10546-006-9048-6, 2006.

Foken, T.: The energy balance closure problem: an overwiew, Ecological Applications, 18, 13511367, doi:10.1890/06-0922.1, 2008a.

Foken, T.: Micrometeorology, 308 pp., Springer Science and Business Media, Berlin, Germany, 2008b.

Foken, T., Mauder, M., Liebethal, C., Wimmer, F., Beyrich, F., Raasch, S., DeBruin, H. A. R., Meijninger, W. M. L., and Bange, J.: Attempt to close the energy balance for the LITFASS2003 experiment, in: 17th Symposium on Boundary Layers and Turbulence, 27th Conference on Agricultural and Forest Meteorology, 17th Conference on Biometeorology and Aerobiology, 2006.

Foken, T., Meixner, F., Falge, E., Zetzsch, C., Serafimovich, A., Bargsten, A., Behrendt, T., Biermann, T., Breuninger, C., and Dix, S.: Coupling processes and exchange of energy and reactive and non-reactive trace gases at a forest siteâĂŞresults of the EGER experiment, Atmospheric Chemistry and Physics, 12, 1923-1950, 2012.

Fuentes, J., Lerdau, M., Atkinson, R., Baldocchi, D., Bottenheim, J., Ciccioli, P., Lamb, B., Geron, C., Gu, L., and Guenther, A.: Biogenic hydrocarbons in the atmospheric boundary layer: a review, Bulletin of the American Meteorological Society, 81, 2000.

Fujita, E., Keislar, R., Stockwell, W., Tanrikulu, S., Ranzieri, A., Moosmuller, H., DuBois, D., Koracin, D., and Zielinska, B.: Central California Ozone Study (CCOS), Volume I: Conceptual Program Plan, Version 2.1, available at www.arb.ca.gov/ccaqs/ccos/ccos.htm., Report, 1999.

Fujita, E., Campbell, D., Keisler, R., Brown, J., Tanrikulu, S., and Ranzieri, A.: Central California Ozone Study (CCOS)-Final report, Volume III: Summary of field operations, technical report, California Air Resource Board, Sacramento, available at http://www.arb.ca.gov/airways/ccos/ccos.htm, Report, technical report, Calif. Air Resour. Board, Sacramento, available at http://www.arb.ca.gov/airways/ccos/ccos.htm, 2001.

Fujita, E., Campbell, D., and Snorradottir, T.: Central California Ozone Study (CCOS) Data Validation, Final Report, California Air Resources Board, Sacramento, CA, available at http://www.arb.ca.gov/airways/ccos/ccos.htm, Report, California Air Resources Board, Sacramento, CA, available at http://www.arb.ca.gov/airways/ccos/ccos.htm, 2005. 
Ganzeveld, L. and Lelieveld, J.: Impact of Amazonian deforestation on atmospheric chemistry, Geophysical Research Letters, 31, L06 105 1-5, 2004.

Ganzeveld, L., Lelieveld, J., Dentener, F., Krol, M., and Roelofs, G.: Atmosphere-biosphere trace gas exchanges simulated with a single-column model, Journal of Geophysical Research D: Atmospheres, 107, XLXV-XLXVI, 2002.

Ganzeveld, L., Klemm, O., RappenglÃijck, B., and Valverde-Canossa, J.: Evaluation of meteorological parameters over a coniferous forest in a single-column chemistry-climate model, Atmospheric Environment, 40, 21-27, 2006.

Ganzeveld, L., Eerdekens, G., Feig, G., Fischer, H., Harder, H., Konigstedt, R., Kubistin, D., Martinez, M., Meixner, F., Scheeren, H., Sinha, V., Taraborrelli, D., Williams, J., Vilà-Guerau De Arellano, J., and Lelieveld, J.: Surface and boundary layer exchanges of volatile organic compounds, nitrogen oxides and ozone during the GABRIEL campaign, Atmospheric Chemistry and Physics, 8, 6223-6243, 2008.

Ganzeveld, L., Bouwman, L., Stehfest, E., Van Vuuren, D., Eickhout, B., and Lelieveld, J.: Impact of future land use and land cover changes on atmospheric chemistry-climate interactions, Journal of Geophysical Research: Atmospheres, 115, 2010.

Gao, W. and Wesely, M.: Numerical modeling of the turbulent fluxes of chemically reactive trace gases in the atmospheric boundary layer, Journal of Applied Meteorology, 33, 835-847, 1994.

Gao, W., Shaw, R., and Paw, K.: Observation of Organized Structure in Turbulent Flow within and above a Forest Canopy, pp. 349-377, Springer Netherlands, doi:10.1007/978-94-009-0975-5 22, 1989.

Gao, W., Wesely, M., and Lee, I.: A numerical study of the effects of air chemistry on fluxes of NO, NO2, and O3 near the surface, Journal of Geophysical Research: Atmospheres, 96, $18761-18769,1991$.

Gao, W., Wesely, M., and Doskey, P.: Numerical modeling of the turbulent diffusion and chemistry of $\mathrm{NO} x, \mathrm{O} 3$, isoprene, and other reactive trace gases in and above a forest canopy, Journal of Geophysical Research: Atmospheres, 98, 18 339-18 353, 1993.

Garratt, J.: Transfer characteristics for a heterogeneous surface of large aerodynamic roughness, Quarterly Journal of the Royal Meteorological Society, 104, 491-502, 1978.

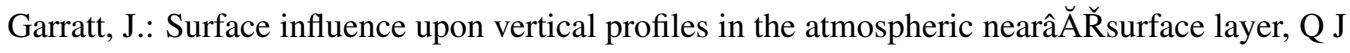
Roy Meteor Soc, 106, 803-819, 1980.

Garratt, J.: The atmospheric boundary layer, Cambridge university press, UK, 1992. 
Gillies, R. and Carlson, T.: Thermal remote sensing of surface soil water content with partial vegetation cover for incorporation into climate models, Journal of Applied Meteorology, 34, 745-756, 1995.

Glaser, K., Vogt, U., Baumbach, G., Volz-Thomas, A., and Geiss, H.: Vertical profiles of O3, NO2, NOx, VOC, and meteorological parameters during the Berlin Ozone Experiment (BERLIOZ) campaign, Journal of Geophysical Research: Atmospheres, 108, 2003.

Goldstein, A., McKay, M., Kurpius, M., Schade, G., Lee, A., Holzinger, R., and Rasmussen, R.: Forest thinning experiment confirms ozone deposition to forest canopy is dominated by reaction with biogenic VOCs, Geophysical Research Letters, 31, 2004.

Goudriaan, J.: Crop micrometeorology : a simulation study, Thesis, URL http: //edepot . wur . nl/166537C1-NN08200, 683, 1977.

Griffin, R., Beckman, P., Talbot, R., Sive, B., and Varner, R.: Deviations from ozone photostationary state during the International Consortium for Atmospheric Research on Transport and Transformation 2004 campaign: Use of measurements and photochemical modeling to assess potential causes, Journal of Geophysical Research: Atmospheres, 112, 2007.

Guenther, A., Geron, C., Pierce, T., Lamb, B., Harley, P., and Fall, R.: Natural emissions of nonmethane volatile organic compounds, carbon monoxide, and oxides of nitrogen from North America, Atmospheric Environment, 34, 2205-2230, 2000.

Gutman, G. and Ignatov, A.: The derivation of the green vegetation fraction from NOAA/AVHRR data for use in numerical weather prediction models, International Journal of remote sensing, 19, 1533-1543, 1998.

Harman, I.: The role of roughness sublayer dynamics within surface exchange schemes, Boundary-Layer Meteorology, 142, 1-20, 2012.

Harman, I. and Finnigan, J.: A simple unified theory for flow in the canopy and roughness sublayer, Boundary-Layer Meteorology, 123, 339-363, 2007.

Harman, I. and Finnigan, J.: Scalar concentration profiles in the canopy and roughness sublayer, Boundary-Layer Meteorology, 129, 323-351, 2008.

Hartogensis, O. and De Bruin, H.: MoninâĂŞObukhov Similarity Functions of the Structure Parameter of Temperature and Turbulent Kinetic Energy Dissipation Rate in the Stable Boundary Layer, Boundary-Layer Meteorology, 116, 253-276, doi:10.1007/s10546-004-2817-1, 2005.

Hayes, TP, K. J. and Wheeler, N.: California surface wind climatology, California Air Resources Board, Aerometric Data Division, Aerometric Projects and Laboratory Branch, Meteorology Section, 1989. 
Högström, U.: Non-dimensional wind and temperature profiles in the atmospheric surface layer: A re-evaluation, pp. 55-78, Springer, 1988.

Högström, U.: Analysis of turbulence structure in the surface layer with a modified similarity formulation for near neutral conditions, Journal of Atmosspheric Science, 47, 1949-1972, 1990.

Högström, U.: Review of Some Basic Characteristics of the Atmospheric Surface Layer, book section 11, pp. 215-246, Springer Netherlands, doi:10.1007/978-94-017-0944-6-11, 1996.

Högström, U., Bergström, H., Smedman, A., Halldin, S., , and Lindroth, A.: Turbulent exchange above a pine forest, I: Fluxes and gradients, Boundary-Layer Meteorology, 49, 197217, doi:10.1007/bf00116411, 1989.

Hollinger, D. and Richardson, A.: Uncertainty in eddy covariance measurements and its application to physiological models, Tree physiology, 25, 873-885, 2005.

Holzinger, R., Lee, A., Paw, K., and Goldstein, U.: Observations of oxidation products above a forest imply biogenic emissions of very reactive compounds, Atmospheric Chemistry and Physics, 5, 67-75, doi:10.5194/acp-5-67-2005, 2005.

Hu, X., Doughty, D., Sanchez, K., Joseph, E., and Fuentes, J.: Ozone variability in the atmospheric boundary layer in Maryland and its implications for vertical transport model, Atmospheric Environment, 46, 354-364, 2012.

Idso, S., Jackson, R., Reginato, R., Kimball, B., and Nakayama, F.: The dependence of bare soil albedo on soil water content, Journal of Applied Meteorology, 14, 109-113, 1975.

Iwata, H., Harazono, Y., and Ueyama, M.: Influence of source/sink distributions on flux-gradient relationships in the roughness sublayer over an open forest canopy under unstable conditions, Boundary-Layer Meteorology, 136, 391-405, 2010.

Jackson, P.: On the displacement height in the logarithmic velocity profile, Journal of Fluid Mechanics, 111, 15-25, 1981.

Jacobs, A., Heusinkveld, B. G., and Holtslag, A. A.: Towards Closing the Surface Energy Budget of a Mid-latitude Grassland, Bound-Lay Meteorol, 126, 125-136, doi:10.1007/s10546-0079209-2, 2007.

Jacobson, M.: Fundamentals of atmospheric modeling, Cambridge University Press, 2005.

Jager, J.: Airborne VOC measurements on board the Zeppelin NT during the PEGASOS campaigns in 2012 deploying the improved Fast-GC-MSD System, Forschungszentrum Jülich, 2014. 
Janssen, R., Vilà-Guerau de Arellano, J., Ganzeveld, L., Kabat, P., Jimenez, J., Farmer, D., van Heerwaarden, C., and Mammarella, I.: Combined effects of surface conditions, boundary layer dynamics and chemistry on diurnal SOA evolution, Atmospheric Chemistry and Physics, 12, 6827-6843, 2012.

Janssen, R., Vilà-Guerau de Arellano, J., Jimenez, J., Ganzeveld, L., Robinson, N., Allan, J., Coe, H., and Pugh, T.: Influence of boundary layer dynamics and isoprene chemistry on the organic aerosol budget in a tropical forest, Journal of Geophysical Research: Atmospheres, 118, 9351-9366, doi:10.1002/jgrd.50672, 2013.

Kaimal, J. and Finnigan, J.: Atmospheric boundary layer flows: their structure and measurement, Oxford University Press Inc, New York, 1994.

Karl, T. and Trenberth, K.: Modern Global Climate Change, Science, 302, 1719-1723, doi:10.1126/science.1090228, 2003.

Karl, T., Potosnak, M., Guenther, A., Clark, D., Walker, J., Herrick, J., and Geron, C.: Exchange processes of volatile organic compounds above a tropical rain forest: Implications for modeling tropospheric chemistry above dense vegetation, Journal of Geophysical Research: Atmospheres, 109, doi:10.1029/2004jd004738, 2004.

Karl, T., Guenther, A., Yokelson, R., Greenberg, J., Potosnak, M., Blake, D., and Artaxo, P.: The tropical forest and fire emissions experiment: Emission, chemistry, and transport of biogenic volatile organic compounds in the lower atmosphere over Amazonia, Journal of Geophysical Research: Atmospheres, 112, 2007.

Karl, T., Guenther, A., Turnipseed, A., Patton, E., and Jardine, K.: Chemical sensing of plant stress at the ecosystem scale, Biogeosciences, 5, 1287-1294, 2008.

Katul, G. and Albertson, J.: An investigation of higher-order closure models for a forested canopy, Boundary-Layer Meteorology, 89, 47-74, 1998.

Katul, G., Hsieh, C., Kuhn, G., Ellsworth, D., and Nie, D.: Turbulent eddy motion at the forestâĂŘatmosphere interface, Journal of Geophysical Research: Atmospheres, 102, 13 409_ $13421,1997$.

Katul, G., Mahrt, L., Poggi, D., and Sanz, C.: One- and two-equation models for canopy turbulence, Boundary-Layer Meteorology, 113, 81-109, 2004.

Kaufman, YJ, T. D. and Boucher, O.: A satellite view of aerosols in the climate system, Nature, 419, 215-223, 2002. 
Klink, K. and Willmott, C.: Influence of soil moisture and surface roughness heterogeneity on modeled climate, Climate Research, 4, 105-18, 1994.

Kochendorfer, J. and RamÃyrez, J.: Ecohydrologic controls on vegetation density and evapotranspiration partitioning across the climatic gradients of the central United States, Hydrology and Earth System Sciences Discussions, 5, 649-700, 2008.

Koster, R., Dirmeyer, P., Guo, Z., Bonan, G., Chan, E., Cox, P., Gordon, C., Kanae, S., Kowalczyk, E., and Lawrence, D.: Regions of strong coupling between soil moisture and precipitation, Science, 305, 1138-1140, 2004.

Kramm, G., Muller, H., Fowler, D., Hofken, K., Meixner, F., and Schaller, E.: A modified profile method for determining the vertical fluxes of NO, NO2, ozone, and HNO3 in the atmospheric surface layer, Journal of atmospheric Chemistry, 13, 265-288, 1991.

Krol, M., Molemaker, M., and VilÃă-Guerau De Arellano, J.: Effects of turbulence and heterogeneous emissions on photochemically active species in the convective boundary layer, Journal of Geophysical Research, 105, 6871-6884, 2000.

Kurpius, M. and Goldstein, A.: Gas-phase chemistry dominates O3 loss to a forest, implying a source of aerosols and hydroxyl radicals to the atmosphere, Geophysical research letters, 30, 2003.

Kustas, W., Anderson, M., Alfieri, J., Prueger, J., Geli, H. M., and Neale, C. M.: Mapping evapotranspiration with high-resolution aircraft imagery over vineyards using one-and twosource modeling schemes, Hydrology and Earth System Sciences, 20, 1523, 2016.

Lamaud, E., Carrara, A., Brunet, Y., Lopez, A., and Druilhet, A.: Ozone fluxes above and within a pine forest canopy in dry and wet conditions, Atmospheric Environment, 36, 77-88, 2002.

Lawrence, D., Thornton, P., Oleson, K., and Bonan, G.: The partitioning of evapotranspiration into transpiration, soil evaporation, and canopy evaporation in a GCM: Impacts on landatmosphere interaction, Journal of Hydrometeorology, 8, 862-880, 2007.

Lean, J. and Rowntree, P.: A GCM simulation of the impact of Amazonian deforestation on climate using an improved canopy representation, Quarterly Journal of the Royal Meteorological Society, 119, 509-530, 1993.

Leclerc, M., Shaw, R., Hartog, G. d., and Neumann, H.: The influence of atmospheric stability on the budgets of the Reynolds stress and turbulent kinetic energy within and above a deciduous forest, Journal of Applied Meteorology, 29, 916-933, 1990. 
Lee, X. and Massman, W.: A perspective on thirty years of the Webb, Pearman and Leuning density corrections, Boundary-Layer Meteorology, 139, 37-59, 2011.

Lefsky, M.: A global forest canopy height map from the Moderate Resolution Imaging Spectroradiometer and the Geoscience Laser Altimeter System, Geophysical Research Letters, 37, doi:10.1029/2010g1043622, 2010.

Lelieveld, J., Butler, T., Crowley, J., Dillon, T., Fischer, H., Ganzeveld, L., Harder, H., Lawrence, M., Martinez, M., Taraborrelli, D., and Williams, J.: Atmospheric oxidation capacity sustained by a tropical forest, Nature, 452, 737-740, 2008.

Lettau, H.: Note on aerodynamic roughness-parameter estimation on the basis of roughnesselement description, Journal of Applied Meteorology, 8, 828-832, 1969.

Li, Y., Barth, M., Chen, G., Patton, E., Kim, S.-W., Wisthaler, A., Mikoviny, T., Fried, A., Clark, R., and Steiner, A.: Large-eddy simulation of biogenic VOC chemistry during the DISCOVERâĂ̌̌AQ 2011 campaign, Journal of Geophysical Research: Atmospheres, 121, 8083-8105, 2016 .

Lilly, D.: Models of cloud-topped mixed layers under a strong inversion, Quarterly Journal of the Royal Meteorological Society, 94, 292-309, 1968.

Logan, J., Staehelin, J., Megretskaia, I., Cammas, J., Thouret, V., Claude, H., De Backer, H., Steinbacher, M., Scheel, H., and StÃijbi, R.: Changes in ozone over Europe: Analysis of ozone measurements from sondes, regular aircraft (MOZAIC) and alpine surface sites, Journal of Geophysical Research: Atmospheres, 117, 2012.

Loreto, F. and Schnitzler, J.-P.: Abiotic stresses and induced BVOCs, Trends in plant science, 15, 154-166, 2010.

Makar, P., Fuentes, J., Wang, D., Staebler, R., and Wiebe, H.: Chemical processing of biogenic hydrocarbons within and above a temperate deciduous forest, Journal of Geophysical Research: Atmospheres, 104, 3581-3603, 1999.

Mannschreck, K., Gilge, S., Plass-Duelmer, C., Fricke, W., and Berresheim, H.: Assessment of the applicability of NO-NO2-O3 photostationary state to long-term measurements at the Hohenpeissenberg GAW Station, Germany, Atmospheric Chemistry and Physics, 4, 12651277, 2004.

Maurer, K., Hardiman, B., Vogel, C., and Bohrer, G.: Canopy-structure effects on surface roughness parameters: Observations in a Great Lakes mixed-deciduous forest, Agricultural and Forest Meteorology, 177, 24-34, 2013. 
Maurer, K., Bohrer, G., Kenny, W., and Ivanov, V.: Large-eddy simulations of surface roughness parameter sensitivity to canopy-structure characteristics, Biogeosciences, 12, 2533-2548, 2015.

Mayor, S.: Observations of Seven Atmospheric Density Current Fronts in Dixon, California, Monthly Weather Review, 139, 1338-1351, 2011.

McCaughey, J. and Saxton, W.: Energy balance storage terms in a mixed forest, Agricultural and Forest Meteorology, 44, 1-18, doi:http://dx.doi.org/10.1016/0168-1923(88)90029-9, 1988.

Meyers, T. and Baldocchi, D.: The budgets of turbulent kinetic energy and Reynolds stress within and above a deciduous forest, Agricultural and Forest Meteorology, 53, 207-222, 1991.

Meyers, T. and Paw, T.: Testing of a higher-order closure model for modeling airflow within and above plant canopies, Boundary-Layer Meteorology, 37, 297-311, 1986.

Meyers, T. and UKT, P.: Modelling the plant canopy micrometeorology with higher-order closure principles, Agricultural and Forest Meteorology, 41, 143-163, 1987.

Mikkelsen, T., Ro-Poulsen, H., Pilegaard, K., Hovmand, M., Jensen, N., Christensen, C., and Hummelshoej, P.: Ozone uptake by an evergreen forest canopy: temporal variation and possible mechanisms, Environmental Pollution, 109, 423-429, 2000.

Min, K.-E., Pusede, S., Browne, E., LaFranchi, B., and Cohen, R.: Eddy covariance fluxes and vertical concentration gradient measurements of NO and NO2 over a ponderosa pine ecosystem: observational evidence for within-canopy chemical removal of NOx, Atmospheric Chemistry and Physics, 14, 5495-5512, 2014.

Moene, F. and Van Dam, C.: Transport in the Atmosphere-Vegetation-Soil Continuum, Cambridge University Press, 32 Avenue of the Americas, New York NY 10013-2473, USA, 2014.

Mölder, M., Grelle, A., Lindroth, A., and Halldin, S.: Flux-profile relationships over a boreal forestâǍTroughness sublayer corrections, Agricultural and Forest Meteorology, 98, 645-658, 1999.

Monahan, A., Rees, T., He, Y., and McFarlane, N.: Multiple regimes of wind, stratification, and turbulence in the stable boundary layer, Journal of the Atmospheric Sciences, 72, 3178-3198, 2015.

Moncrieff, J., Clement, R., Finnigan, J., and Meyers, T.: Averaging, detrending, and filtering of eddy covariance time series, pp. 7-31, Springer, 2005.

Monin, A. and Obukhov, A.: Basic laws of turbulent mixing in the surface layer of the atmosphere, Contrib. Geophys. Inst. Acad. Sci. USSR, 151, 163-187, 1954. 
Monson, R. and Baldocchi, D.: Terrestrial biosphere-atmosphere fluxes, Cambridge University Press, 2014.

Moore, K., Fitzjarrald, D., Sakai, R., Goulden, M., Munger, J. W., and Wofsy, S.: Seasonal variation in radiative and turbulent exchange at a deciduous forest in central Massachusetts, Journal of Applied Meteorology, 35, 122-134, 1996.

Morris, G., Ford, B., RappenglÃijck, B., Thompson, A., Mefferd, A., Ngan, F., and Lefer, B.: An evaluation of the interaction of morning residual layer and afternoon mixed layer ozone in Houston using ozonesonde data, Atmospheric Environment, 44, 4024-4034, 2010.

Munn, R., Gao, W., Shaw, R., and UKT, P.: Observation of Organized Structure in Turbulent Flow within and above a Forest Canopy, pp. 349-377, Springer Netherlands, doi:10.1007/97894-009-0975-5-22, 1989.

Murphy, J., Day, D., Cleary, P., Wooldridge, P., Millet, D., Goldstein, A., and Cohen, R.: The weekend effect within and downwind of Sacramento: Part 2. Observational evidence for chemical and dynamical contributions, Atmospheric Chemistry and Physics Discussions, 6, 11971$12019,2006$.

Murphy, J., Day, D., Cleary, P., Wooldridge, P., Millet, D., Goldstein, A., and Cohen, R.: The weekend effect within and downwind of Sacramento-Part 1: Observations of ozone, nitrogen oxides, and VOC reactivity, Atmospheric Chemistry and Physics, 7, 5327-5339, 2007.

Nicholson, S.: Land surface atmosphere interaction, Prog. Phys. Geogr, 12, 36-65, 1988.

Ohta, T., Suzuki, K., Kodama, Y., Kubota, J., Kominami, Y., and Nakai, Y.: Characteristics of the heat balance above the canopies of evergreen and deciduous forests during the snowy season, Hydrological Processes, 13, 2383-2394, doi:10.1002/(sici)10991085(199910)13:14/15<2383::aid-hyp872>3.0.co;2-s, 1999.

Oliphant, A., Grimmond, C., Zutter, H., Schmid, H., Su, H., Scott, S., Offerle, B., Randolph, J., and Ehman, J.: Heat storage and energy balance fluxes for a temperate deciduous forest, Agricultural and Forest Meteorology, 126, 185-201, 2004.

Oliveira, P., Acevedo, O., Moraes, O., Zimermann, H., and Teichrieb, C.: Nocturnal intermittent coupling between the interior of a pine forest and the air above it, Boundary-Layer Meteorology, 146, 45-64, 2013.

Ouwersloot, H. and Vilà-Guerau de Arellano, J.: Analytical solution for the convectively-mixed atmospheric boundary layer, Boundary-Layer Meteorology, 148, 557-583, 2013. 
Ouwersloot, H., Vilà-Guerau de Arellano, J., van Heerwaarden, C., Ganzeveld, L., Krol, M., and Lelieveld, J.: On the segregation of chemical species in a clear boundary layer over heterogeneous land surfaces, Atmospheric chemistry and physics, 11, 10 681-10 704, 2011.

Ouwersloot, H., Vilà-Guerau de Arellano, J., N“̈olscher, A., Krol, M., Ganzeveld, L., Breitenberger, C., Mammarella, I., Williams, J., and Lelieveld, J.: Characterization of a boreal convective boundary layer and its impact on atmospheric chemistry during HUMPPA-COPEC-2010, Atmos Chem Phys, 12, 9335-9353, doi:10.5194/acp-12-9335-2012, 2012.

Ouwersloot, H., Moene, A., Attema, J., and Vilà-Guerau de Arellano, J.: Large-Eddy Simulation Comparison of Neutral Flow Over a Canopy: Sensitivities to Physical and Numerical Conditions, and Similarity to Other Representations, Boundary-Layer Meteorology, pp. 1-19, 2016.

Panofsky, H., Tennekes, H., Lenschow, D. H., and Wyngaard, J.: The characteristics of turbulent velocity components in the surface layer under convective conditions, Boundary-Layer Meteorology, 11, 355-361, 1977.

Patton, E. and Finnigan, J.: Canopy Turbulence, vol. Overview and Fundamentals, pp. 301 - 327 , CRC Press, Taylor and Francis Group, The United States of America, 2013.

Patton, E., Sullivan, P., and Davis, K.: The influence of a forest canopy on top-down and bottomup diffusion in the planetary boundary layer, Quart. J. Roy. Meteor. Soc., 129, 1415-1434, doi:10.1256/qj.01.175, 2003.

Patton, E., Sullivan, P., and Moeng, C.-H.: The Influence of Idealized Heterogeneity on Wet and Dry Planetary Boundary Layers Coupled to the Land Surface, Journal of the Atmospheric Sciences, 62, 2078-2097, doi:doi:10.1175/JAS3465.1, 2005.

Patton, E., Horst, T., Sullivan, P., Lenschow, D., Oncley, S., Brown, W., Burns, S., Guenther, A., Held, A., Karl, T., Mayor, S., Rizzo, L., Spuler, S., Sun, J., Turnipseed, A., Allwine, E., Edburg, S., Lamb, B., Avissar, R., Calhoun, R., Kleissl, J., Massman, W., Paw, U., and Weil, J.: The Canopy Horizontal Array Turbulence Study, Bulletin of the American Meteorological Society, 92, 593-611, doi:10.1175/2010bams2614.1, 2010.

Patton, E., Sullivan, P., Shaw, R., Finnigan, J., and Weil, J.: Atmospheric Stability Influences on Coupled Boundary Layer and Canopy Turbulence, Journal of the Atmospheric Sciences, 73, 1621-1647, 2016.

Patton, E. G., Davis, K. J., Barth, M. C., and Sullivan, P. P.: Decaying scalars emitted by a forest canopy: a numerical study, Boundary-Layer Meteorology, 100, 91-129, 2001. 
Physick, W. and Garratt, J.: Incorporation of a high-roughness lower boundary into a mesoscale model for studies of dry deposition over complex terrain, Bound-Lay Meteorol, 74, 55-71, 1995.

Pielke, R., Cotton, W., Walko, R. e. a., Tremback, C. J., Lyons, W. A., Grasso, L., Nicholls, M., Moran, M., Wesley, D., and Lee, T.: A comprehensive meteorological modeling systemâĂŤRAMS, Meteorology and Atmospheric Physics, 49, 69-91, 1992.

Pielke, R., Avissar, R., Raupach, M., Dolman, A., Zeng, X., and Denning, A.: Interactions between the atmosphere and terrestrial ecosystems: influence on weather and climate, Global Change Biology, 4, 461-475, 1998.

Pietersen, H., Vilà-Guerau de Arellano, J., Augustin, P., van de Boer, A., de Coster, O., Delbarre, H., Durand, P., Fourmentin, M., Gioli, B., Hartogensis, O., Lohou, F., Lothon, M., Ouwersloot, H., Pino, D., and Reuder, J.: Study of a prototypical convective boundary layer observed during BLLAST: contributions by large-scale forcings, Atmospheric Chemistry and Physics, 15, 4241-4257, doi:10.5194/acp-15-4241-2015, aCP, 2015.

Pilegaard, K., HummelshÃÿj, P., and Jensen, N.: Nitric oxide emission from a Norway spruce forest floor, Journal of Geophysical Research: Atmospheres, 104, 3433-3445, 1999.

Pino, D., Vilà-Guerau de Arellano, J., and Duynkerke, P.: The Contribution of Shear to the Evolution of a Convective Boundary Layer, Journal of the Atmospheric Sciences, 60, 1913-1926, doi:10.1175/1520-0469(2003)060<1913:tcostt>2.0.co;2, 2003.

Pinto, D., Tiiva, P., Miettinen, P., Joutsensaari, J., Kokkola, H., Nerg, A.-M., Laaksonen, A., and Holopainen, J.: The effects of increasing atmospheric ozone on biogenic monoterpene profiles and the formation of secondary aerosols, Atmospheric Environment, 41, 4877-4887, 2007.

Poggi, D., Porporato, A., Ridolfi, L., Albertson, J., and Katul, G.: The effect of vegetation density on canopy sub-layer turbulence, Boundary-Layer Meteorology, 111, 565-587, 2004.

Pope, S.: Turbulent flows, Cambridge University Press, 2000.

Rannik, U., Keronen, P., Hari, P., and Vesala, T.: Estimation of forest-atmosphere CO2 exchange by eddy covariance and profile techniques, Agricultural and Forest Meteorology, 126, 141-155, 2004.

Raupach, M.: Anomalies in Flux-Gradient Relationships Over Forest, Boundary-Layer Meteorology, 16, 467-486, doi:10.1007/bf03335385, 1979.

Raupach, M.: Biometeorology Applying Lagrangian fluid mechanics to infer scalar source distributions from concentration profiles in plant canopies, Agricultural and Forest Meteorology, 47, 85-108, doi:http://dx.doi.org/10.1016/0168-1923(89)90089-0, 1989a. 
Raupach, M.: A practical Lagrangian method for relating scalar concentrations to source distributions in vegetation canopies, Quarterly Journal of the Royal Meteorological Society, 115, 609-632, doi:10.1002/qj.49711548710, 1989 b.

Raupach, M.: Drag and drag partition on rough surfaces, Boundary-Layer Meteorol, 60, 375-395, doi:10.1007/bf00155203, boundary-Layer Meteorol, 1992.

Raupach, M.: Simplified expressions for vegetation roughness length and zero-plane displacement as functions of canopy height and area index, Boundary-Layer Meteorology, 71, 211-216, 1994.

Raupach, M. and Thom, A.: Turbulence in and above plant canopies, Annual Review of Fluid Mechanics, volume 13, pp. 97-129, 1981.

Raupach, M., Thom, A., and Edwards, I.: A wind-tunnel study of turbulent flow close to regularly arrayed rough surfaces, Boundary-Layer Meteorology, 18, 373-397, 1980.

Raupach, M., Coppin, P., and Legg, B.: Experiments on scalar dispersion within a model plant canopy part I: The turbulence structure, Boundary-Layer Meteorology, 35, 21-52, 1986.

Raupach, M., Finnigan, J., and Brunet, Y.: Coherent eddies and turbulence in vegetation canopies: the mixing-layer analogy, Boundary-Layer Meteorology, 78, 351-382, 1996.

Rogers, M.: The structure of a passive scalar field with a uniform mean gradient in rapidly sheared homogeneous turbulent flow, Physics of Fluids A: Fluid Dynamics (1989-1993), 3, 144-154, 1991.

Rohrer, F. and Berresheim, H.: Strong correlation between levels of tropospheric hydroxyl radicals and solar ultraviolet radiation, Nature, 442, 184-187, 2006.

Running, S., Nemani, R., Heinsch, F., Zhao, M., Reeves, M., and Hashimoto, H.: A continuous satellite-derived measure of global terrestrial primary production, Bioscience, 54, 547-560, 2004.

Santos, D., Acevedo, O., Chamecki, M., Fuentes, J., Gerken, T., and Stoy, P.: Temporal Scales of the Nocturnal Flow Within and Above a Forest Canopy in Amazonia, Boundary-Layer Meteorology, pp. 1-26, 2016.

Schmid, H.: Footprint modeling for vegetation atmosphere exchange studies: a review and perspective, Agricultural and Forest Meteorology, 113, 159-183, 2002.

Schowengerdt, R.: Remote sensing: models and methods for image processing, Academic press, San Diefo, California, USA, third edition edn., 2006. 
Schumann, U.: Large-eddy simulation of turbulent diffusion with chemical reactions in the convective boundary layer, Atmospheric Environment (1967), 23, 1713-1727, 1989.

Seinfeld, J. and Pandis, S.: Atmospheric chemistry and physics: from air pollution to climate change, John Wiley and Sons, 2016.

Seok, B., Helmig, D., Ganzeveld, L., Williams, M., and Vogel, C.: Dynamics of nitrogen oxides and ozone above and within a mixed hardwood forest in northern Michigan, Atmospheric Chemistry and Physics, 13, 7301, 2013.

Shapkalijevski, M., Moene, A., Ouwersloot, H., Patton, E., and Vilà-Guerau de Arellano, J.: Influence of Canopy Seasonal Changes on Turbulence Parameterization within the Roughness Sublayer over an Orchard Canopy, Journal of Applied Meteorology and Climatology, 55, 13911407, doi:doi:10.1175/JAMC-D-15-0205.1, 2016.

Shapkalijevski, M., Ouwersloot, G., Moene, F., and Arellano, Vilà-Guerau de Arellano, J.: Integrating canopy and large-scale atmospheric effects in convective boundary-layer dynamics during CHATS experiment, Atmospheric Chemistry and Physics, 3, 1623-1640, doi:10.5194/acp17-1623-2017, 2017.

Shaw, R. and Schumann, U.: Large-eddy simulation of turbulent flow above and within a forest, Boundary-Layer Meteorology, 61, 47-64, doi:10.1007/bf02033994, 1992.

Shaw, R., Finnigan, J., Patton, E., and Fitzmaurice, L.: Eddy structure near plant canopy interface, in: 17th Symposium on Boundary Layers and Turbulence, Conference Proceeding. San Diego, Canada. p. J, vol. 2, 2006.

Sillman, S.: The relation between ozone, NOx and hydrocarbons in urban and polluted rural environments, Atmospheric Environment, 33, 1821-1845, doi:http://dx.doi.org/10.1016/S13522310(98)00345-8, 1999.

Simard, M., Pinto, N., Fisher, J., and Baccini, A. C. G.: Mapping forest canopy height globally with spaceborne lidar, Journal of Geophysical Research: Biogeosciences, 116, doi:10.1029/2011jg001708, 2011.

Simpson, I., Thurtell, G., Neumann, H., Den Hartog, G., and Edwards, G.: The validity of similarity theory in the roughness sublayer above forests, Boundary-Layer Meteorology, 87, 69-99, 1998.

Skamarock, W., Klemp, J., Dudhia, J., Gill, D., Barker, D., Wang, W., and Powers, J.: A description of the advanced research WRF version 2, Report, DTIC Document, 2005. 
Sogachev, A., Menzhulin, G., Heimann, M., and Lloyd, J.: A simple three-dimensional canopyPlanetary boundary layer simulation model for scalar concentrations and fluxes, Tellus, Series B: Chemical and Physical Meteorology, 54, 784-819, 2002.

Sogachev, A., Kelly, M., and Leclerc, M.: Consistent Two-Equation Closure Modelling for Atmospheric Research: Buoyancy and Vegetation Implementations, Boundary-Layer Meteorology, pp. 1-21, 2012.

Stockwell, W. and Goliff, W.: Measurement of actinic flux and the calculation of photolysis rate parameters for the Central California Ozone Study, Atmospheric Environment, 38, 5169-5177, 2004.

Stull, R.: An introduction to boundary layer meteorology, vol. 13, Springer Science and Business Media, doi:10.1007/978-94-009-3027-8, 1988.

Sullivan, P., Moeng, C.-H., Stevens, B., Lenschow, D. H., and Mayor, S.: Structure of the entrainment zone capping the convective atmospheric boundary layer, Journal of the Atmospheric Sciences, 55, 3042-3064, 1998.

Sun, J. and Massman, W.: Ozone transport during the California ozone deposition experiment, Journal of Geophysical Research, 104, 11 939-11 948, 1999.

Sykes, R., Parker, S., Henn, D., and Lewellen, W.: Turbulent mixing with chemical reaction in the planetary boundary layer, Journal of Applied Meteorology, 33, 825-834, 1994.

Tennekes, H.: Similarity relations, scaling laws and spectral dynamics, pp. 37-68, Springer, 1984.

Tennekes, H. and Driedonks, A.: Basic entrainment equations for the atmospheric boundary layer, Boundary-Layer Meteorology, 20, 515-531, 1981.

Tennekes, H. and Lumley, J.: A first course in turbulence, MIT press, 1972.

Thom, A.: Momentum absorption by vegetation, Quarterly Journal of the Royal Meteorological Society, 97, 414-428, doi:10.1002/qj.49709741404, 1971.

Thom, A., Stewart, J., Oliver, H., and Gash, J.: Comparison of aerodynamic and energy budget estimates of fluxes over a pine forest, Quarterly Journal of the Royal Meteorological Society, 101, 93-105, 1975.

Thomas, C. and Foken, T.: Flux contribution of coherent structures and its implications for the exchange of energy and matter in a tall spruce canopy, Boundary-Layer Meteorology, 123, 317-337, doi:10.1007/s10546-006-9144-7, 2007. 
Thornton, J., Wooldridge, P., Cohen, R., Martinez, M., Harder, H., Brune, W., Williams, E., Roberts, J., Fehsenfeld, F., and Hall, S.: Ozone production rates as a function of NOx abundances and HOx production rates in the Nashville urban plume, Journal of Geophysical Research: Atmospheres, 107, 2002.

Van Gorsel, E., Harman, I., Finnigan, J., and Leuning, R.: Decoupling of air flow above and in plant canopies and gravity waves affect micrometeorological estimates of net scalar exchange, Agricultural and Forest Meteorology, 151, 927-933, 2011.

van Heerwaarden, C., Vilà-Guerau de Arellano, J., Moene, A., and Holtslag, A.: Interactions between dry-air entrainment, surface evaporation and convective boundary-layer development, Quarterly Journal of the Royal Meteorological Society, 135, 1277-1291, doi:10.1002/qj.431, 2009.

van Heerwaarden, C., Vilà-Guerau de Arellano, J., Gounou, A., Guichard, F., and Couvreux, F.: Understanding the Daily Cycle of Evapotranspiration: A Method to Quantify the Influence of Forcings and Feedbacks, Journal of Hydrometeorology, 11, 1405-1422, 2010.

van Stratum, B., Vilà-Guerau de Arellano, J., Ouwersloot, H., van den Dries, K., van Laar, T., Martinez, M., Lelieveld, J., Diesch, J., Drewnick, F., Fischer, H., Hosaynali Beygi, Z., Harder, H., Regelin, E., Sinha, V., Adame, J., Sörgel, M., Sander, R., Bozem, H., Song, W., Williams, J., and Yassaa, N.: Case study of the diurnal variability of chemically active species with respect to boundary layer dynamics during DOMINO, Atmospheric Chemistry and Physics, 12, 5329-5341, doi:10.5194/acp-12-5329-2012, aCP, 2012.

Vercauteren, N. and Klein, R.: A clustering method to characterize intermittent bursts of turbulence and interaction with submesomotions in the stable boundary layer, Journal of the Atmospheric Sciences, 72, 1504-1517, 2015.

Vilà-Guerau Arellano, J., Duynkerke, P., and Zeller, K.: Atmospheric surface layer similarity theory applied to chemically reactive species, Journal of Geophysical Reseasrch: Atmosphere, 100, 1397-1408, 1995.

Vilà-Guerau de Arellano, J.: Bridging the Gap Between Atmospheric Physics and Chemistry in Studies of Small-Scale Turbulence, Bulletin of the American Meteorological Society, 84, 51-56, doi:10.1175/bams-84-1-51, 2003.

Vilà-Guerau de Arellano, J. and Casso-torralba, P.: The radiation and energy budget in mesoscale models: an observational study case, Fisica de la Tierra, 19, 117-132, 2008.

Vilà-Guerau de Arellano, J. and Duynkerke, P.: Influence of chemistry on the flux-gradient relationships for the NO-O3-NO2 system, Boundary-Layer Meteorology, 61, 375-387, 1992. 
Vilà-Guerau de Arellano, J. and Duynkerke, P.: Second-order closure study of the covariance between chemically reactive species in the surface layer, Journal of Atmospheric Chemistry, 16, 145-155, 1993.

Vilà-Guerau de Arellano, J., Dries, K., and Pino, D.: On inferring isoprene emission surface flux from atmospheric boundary layer concentration measurements, Atmospheric Chemistry and Physics, 9, 3629-3640, 2009.

Vilà-Guerau de Arellano, J., Patton, E., Karl, T., van den Dries, K., Barth, M., and Orlando, J.: The role of boundary layer dynamics on the diurnal evolution of isoprene and the hydroxyl radical over tropical forests, Journal of Geophysical Research: Atmospheres, 116, 2011.

Vilà-Guerau de Arellano, J., van Heerwaarden, C., and Lelieveld, J.: Modelled suppression of boundary-layer clouds by plants in a CO2-rich atmosphere, Nature Geoscience, 5, 701-704, 2012.

Vilà-Guerau de Arellano, J., van Heerwaarden, C., van Stratum, B., and van den Dries, K.: Atmospheric boundary layer: Integrating air chemistry and land interactions, Cambridge University Press, New York, USA, 2015.

Viterbo, P. and Beljaars, A.: An improved land surface parameterization scheme in the ECMWF model and its validation, Journal of Climate, 8, 2716-2748, 1995.

Volz-Thomas, A., Pätz, H., Houben, N., Konrad, S., Mihelcic, D., Klüpfel, T., and Perner, D.: Inorganic trace gases and peroxy radicals during BERLIOZ at Pabstthum: An investigation of the photostationary state of NOx and O3, Journal of Geophysical Research: Atmospheres, 108, 2003.

Wallace, J. and Hobbs, P.: Atmospheric science: an introductory survey, vol. 92, Academic press, New York, 2006.

Webb, E., Pearman, G., and Leuning, R.: Correction of flux measurements for density effects due to heat and water vapour transfer, Quarterly Journal of the Royal Meteorological Society, 106, 85-100, 1980.

Weligepolage, K., Gieske, A. S. M., van der Tol, C., Timmermans, J., and Su, Z.: Effect of sub-layer corrections on the roughness parameterization of a Douglas fir forest, Agricultural and Forest Meteorology, 162â̆̆Ş163, 115-126, doi:http://dx.doi.org/10.1016/j.agrformet.2012.04.017, 2012.

Wesely, M. and Hicks, B.: Some factors that affect the deposition rates of sulfur dioxide and similar gases on vegetation, Journal of the Air Pollution Control Association, 27, 1110-1116, 1977. 
Wesely, M., Sisterson, D., Hart, R., Drapcho, D., and Lee, I.: Observations of nitric oxide fluxes over grass, Journal of Atmospheric Chemistry, 9, 447-463, 1989.

Wilcox, D.: Turbulence modeling for CFD, vol. 2, DCW industries La Canada, CA, 1998.

Wilczak, J., Oncley, S., and Stage, S.: Sonic anemometer tilt correction algorithms, BoundaryLayer Meteorology, 99, 127-150, 2001.

Willmott, C. and Matsuura, K.: Advantages of the mean absolute error (MAE) over the root mean square error (RMSE) in assessing average model performance, Climate research, 30, 79-82, 2005.

Wilson, J.: A second-order closure model for flow through vegetation, Boundary-Layer Meteorology, 42, 371-392, 1988.

Wilson, J.: Turbulent transport within the plant canopy, Estimation of Areal Evapotranspiration, 177, 43-80, 1989.

Wilson, M., Henderson-Sellers, A., Dickinson, R., and Kennedy, P.: Sensitivity of the Biosphere-Atmosphere Transfer Scheme (BATS) to the Inclusion of Variable Soil Characteristics, Journal of Climate and Applied Meteorology, 26, 341-362, doi:doi:10.1175/15200450(1987)026<0341:SOTBTS>2.0.CO;2, 1987.

Wilson, N. and Shaw, R.: A higher order closure model for canopy flow, Journal of Applied Meteorology, 16, 1197-1205, 1977.

Wyngaard, J. and Coté, O.: The Budgets of Turbulent Kinetic Energy and Temperature Variance in the Atmospheric Surface Layer, Journal of Atmospheric Science, 28, 190-201, doi:10.1175/1520-0469(1971)028<0190:tbotke>2.0.co;2, 1971.

Wyngaard, J., Bach Jr, W., Burk, S., Cotton, W., and Ferziger, J.: Large-Eddy Simulation. Guidelines for Its Application to Planetary Boundary Layer Research, Report, DTIC Document, 1984.

Wyngaard, J. C.: Turbulence in the Atmosphere, Cambridge University Press, 2010.

Yamada, N.: Model for the pressure terms in the equations for second-order turbulence moment, and its application to the atmospheric surface layer, Journal of the Meteorological Society Japan, 63, 695-701, 1985.

Yang, J., Honrath, R., Peterson, M., Parrish, D., and Warshawsky, M.: Photostationary state deviationâĂŞestimated peroxy radicals and their implications for HOx and ozone photochemistry at a remote northern Atlantic coastal site, Journal of Geophysical Research: Atmospheres, 109, 2004. 
Zaremba, L. and Carroll, J.: Summer Wind Flow Regimes over the Sacramento Valley, Journal of Applied Meteorology, 38, 1463-1473, doi:doi:10.1175/15200450(1999)038<1463:SWFROT>2.0.CO;2, 1999.

Zeller, K.: Eddy Diffusivities for Sensible Heat, Ozone and Momentum from Eddy Correlation and Gradient Measurements, 1990.

Zeman, O.: Progress in the modeling of planetary boundary layers, Annual Review of Fluid Mechanics, 13, 253-272, 1981.

Zhong, S., Whiteman, C., and Bian, X.: Diurnal Evolution of Three-Dimensional Wind and Temperature Structure in California's Central Valley, Journal of Applied Meteorology, 43, 16791699, doi:doi:10.1175/JAM2154.1, 2004.

Zilitinkevich, S.: Dynamics of the Atmospheric Boundary Layer (in Russian), Gidrometeoizdat., Leningrad, USSR, 1970.

Zilitinkevich, S., Hunt, J., Esau, I. N., Grachev, A., Lalas, D., Akylas, E., Tombrou, M., Fairall, C., Fernando, H., and Baklanov, A.: The influence of large convective eddies on the surfaceâĂ ̌llayer turbulence, Quarterly Journal of the Royal Meteorological Society, 132, 14261456, 2006.

Zilitinkevich, S., Mammarella, I., Baklanov, A., and Joffre, S.: The effect of stratification on the aerodynamic roughness length and displacement height, Boundary-Layer Meteorology, 129, 179-190, 2008.

Zilitinkevich, S., Elperin, T., Kleeorin, N., Rogachevskii, I., and Esau, I.: A Hierarchy of Energyand Flux-Budget (EFB) Turbulence Closure Models for Stably-Stratified Geophysical Flows, Boundary-Layer Meteorology, 146, 341-373, 2013. 


\section{Acknowledgments}

The last paragraphes in this $\mathrm{PhD}$ thesis I am going to spend to thank the people that were supporting me during this process.

First of all, I would like to express my gratitude to my supervisory team. The amazing supervisory team behind this successfully finished $\mathrm{PhD}$ work includes: Jordi, Maarten, Arnold and Huug. Thank you all for the pleasant working environment that each of you created in this team and for all your individual support. Jordi, thank you for being the 'selector' of the team, a great supervisor, teacher, and most importantly, a great person. I have heard you were saying that I never complain about the hard work. How can I complain when whenever I am sending you a document to be checked on Saturday evening, I am receiving comments on Sunday morning (the same weekend)? It has been my pleasure to work with you, and I am looking forward to continue this collaboration in the future. Maarten, thank you for being my first promoter. Especially I would like to thank you for having the willingness, the knowledge and the temper to help me, and support me, in the most critical and difficult moments during my $\mathrm{PhD}$. Arnold, thank you for your contribution and help when dealing with the data access, processing and analyzing the data, but also for all the scientific, in depth, discussions. I truly enjoyed them. Huug, I highly appreciate your contribution in this $\mathrm{PhD}$ project. Thank you for helping me with the codes of the models, but also for the very useful comments on my scientific and English writing.

Next, I would like to thank to the people that were partially involved or were indirectly supporting this PhD project. Ivone and Laurens, thank you for giving me the opportunity to start this $\mathrm{PhD}$, and thank you for all scientific discussions in the first part of the project. Special thanks goes to Nad Patton for the his great contribution in providing the observational (CHATS) data set, but also for often being a non-official, but extremely useful, referee of our manuscripts. I highly appreciate the support and the patience of Nikki and Prof. Klein (from FU-Berlin) during the last phase of my $\mathrm{PhD}$, and therefore I am thankful to them.

As a part of three scientific groups (MAQ, WSG (ESS), and MPIC-Reactive chemistry), I met and collaborated with a number of colleagues. Here I would like to thank all of them. Although not sharing the same working environment, I would like to thank my colleagues and friends from the Meteorology and Air Quality group for always being welcoming and friendly. Then, I would like to thank to my colleagues from the Water Systems and Global Change (previously Earth 
System Science) group for making my stay in the office pleasent, and the working environment enjoyable. Especially, I would like to acknowledge the beautiful $\mathrm{PhD}$ family in this group (ex and current $\mathrm{PhD}$ colleagues and friends) and the great time and moments spent together in- and outside the office. Finally, many thanks to the colleagues from the reactive chemistry group at the Max-Plank Institute for Chemistry in Mainz, with whom I collaborated during the first phase of this project.

It is my honor to have Francesco and Chiel next to me as my paranymphs on the defence day. Thank you to both of you for being my friends in many nice moments, and supporting me during the difficult periods.

At this point, I would like to thank all my friends that I met while living in Wageningen. Ele and Franci, thank you for being my second family. Jan, Stefano, Vuk, Pippo, Samba, Ire, Simo, Ama, Max, Natalia, Oleg, Natasa, Bastjen, Milos, Maja, Kostas and Maria, Dimitri, Edo, Eva, Tom, Vale, Aleksandra, Jana, Berent, Jelena and Florian, Rui, Andrijana, Bram (with the family), Jura, Anika, Veronika, Marjut, it has been a pleasure meeting you, and sharing our free time together on nice dinners, concerts, birthday and wedding celebrations, soccer and ice-hockey games, or simply going out for a coffee.

Finally, I would like to thank my family. Mother, father and sister, thank you for all the support you are providing all these years; you are always making me proud. I dedicate this work to you my dearest Mila and Luka. 



\section{Certificate}

\section{Metodija M. Shapkalijevski}

born May $6^{\text {st }}, 1981$ in Skopje (Macedonia, SFR Yugoslavia)

has successfully attended the programme of the International Max Planck Research School for Atmospheric Chemistry and Physics

\section{Overall grading: Very Good}

\section{Subjects:}

Credits:

\begin{tabular}{lr} 
IMPRS Days 2012 & 1 \\
Physical Modeling & 6 \\
Introduction into Multi-Layer Canopy Chemistry Exchange Model & 1 \\
Boundary-layer processes & 1 \\
COST-ECLAIRE Workshop & 2 \\
Various Research Schools, Oral and Poster Presentations as listed & 15 \\
\cline { 2 - 2 } & $26^{*}$
\end{tabular}

* minimum 12 credit points 


\section{Additional activities of Dr. Metodija M. Shapkalijevski within the framework of the International Max Planck Research School included:}

\section{Research Schools}

- Bridging the Gap-Between Atmospheric Scales, Wageningen University and Research Centre, Wageningen (The Netherlands), October $8^{\text {th }}-12^{\text {th }}$, 2012

- IMPRS Autumn School on Scales and Patterns in the Earth System, Max Planck Institute for the Physics of Complex Systems, Dresden (Germany), November $5^{\text {th }}-9^{\text {th }}, 2012$

\section{Oral presentations}

- Turbulent exchange of reactive compounds in vegetation canopies, IMPRS Days 2012, Heidelberg/Mainz (Germany), June $17^{\mathrm{th}}-21^{\mathrm{st}}, 2012$

- Influence of canopy phenology changes on turbulence above tall deciduous canopy, European Geosciences Union (EGU) Conference (General Assembly), Vienna (Austria), April 12 $2^{\text {th }}-16^{\text {th }}, 2015$

- The role of small canopy-scale turbulence and large meso-scale forcing on convective boundary-layer dynamics, Buys-Ballot Research School for Fundamental Processes in the Climate System (BBOS) Symposium, Renesse (The Netherlands), October $28^{\text {th }}-30^{\text {th }}, 2015$

- Integrating canopy and large-scale atmospheric effects in the convective boundary-layer dynamics and chemistry during CHATS experiment, $32^{\text {nd }}$ Conference on Agricultural and Forest Meteorology, $22^{\text {nd }}$ Symposium on Boundary Layers and Turbulence, $3^{\text {rd }}$ Conference on Biogeosciences, Salt-Lake City, Utah (USA), June $20^{\text {th }}-24^{\text {th }}, 2016$

\section{Poster presentations}

- Turbulent exchange of reactive compounds in vegetation canopy, IMPRS Autumn School on Scales and Patterns in the Earth System, Dresden (Germany), November $5^{\text {th }}-9^{\text {th }}, 2012$ 


\section{Soft Skills}

- Workshop on Writing your own PhD proposal within 6 months after the start of your PhD study, Wageningen University and Research Centre, Wageningen (The Netherlands), 2013

\section{Teaching Assistance}

- Co-Supervisor of MSc student/thesis (6 months), Wageningen University and Research Centre, Wageningen (The Netherlands), 2015/2016

- Organising monthly PhD meetings (scientific, intervision), Wageningen University and Research Centre, Wageningen (The Netherlands), 2015/2016

- Workshop on Introduction into Multi-Layer Canopy Chemistry Exchange Model: MLC CHEM, Teaching Assistant, Max Planck Institute for Chemistry, Mainz (Germany), January $15^{\text {th }}-16^{\text {th }}, 2013$

\section{Publications}

- Shapkalijevski, M., A. F. Moene, H. G. Ouwersloot, E. Patton, and J. Vilà-Guerau de Arellano, 2016: Influence of canopy seasonal changes on turbulence parameterization within the roughness sublayer over an orchard canopy. Journal of Applied Meteorology and Climatology, 55, 1391-1407, doi:10.1175/JAMC-D-15-0205.1.

- Shapkalijevski, M. M., Ouwersloot, H. G., Moene, A. F., and de Arrellano, J. V.-G.: Integrating canopy and large-scale effects in the convective boundary-layer dynamics during the CHATS experiment, Atmos. Chem. Phys., 17, 1623-1640, doi:10.5194/acp-17-1623-2017, 2017. 


\title{
SENSE
}

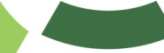

Netherlands Research School for the

Socio-Economic and Natural Sciences of the Environment

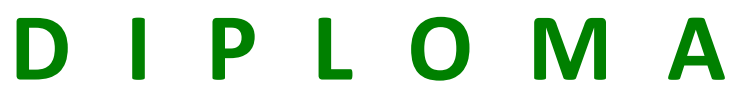

For specialised PhD training

The Netherlands Research School for the Socio-Economic and Natural Sciences of the Environment (SENSE) declares that

\section{Metodija M. Shapkalijevski}

born on 6 May 1981 in Skopje, Macedonia (SFR Yugoslavia)

\author{
has successfully fulfilled all requirements of the \\ Educational Programme of SENSE.
}

Wageningen, 1 May 2017

the Chairman of the SENSE board

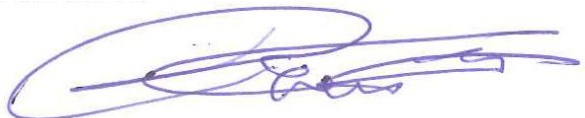

Prof. dr. Huub Rijnaarts the SENSE Director of Education

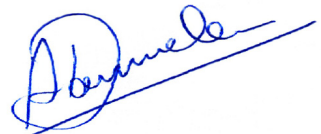

Dr. Ad van Dommelen 


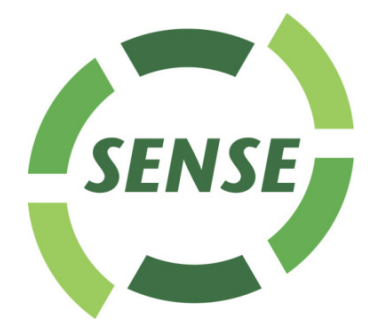

The SENSE Research School declares that Mr Metodija M. Shapkalijevski has successfully fulfilled all requirements of the Educational PhD Programme of SENSE with a work load of $40.0 \mathrm{EC}$, including the following activities:

\section{$\underline{\text { SENSE PhD Courses }}$}

- Environmental research in context (2012)

- Research in context activity: 'Organising monthly scientific and intervision PhD meetings for the department of Water Systems and Global Change'(2015-2016)

\section{Other PhD and Advanced MSc Courses}

- Physical Modelling, Wageningen University (2012)

- Bridging the scale gap between atmospheric scales, Wageningen University (2013)

- Boundary-layer processes, Wageningen University (2013)

\section{External training at a foreign research institute}

- COST ECLAIRE Workshop, French National Institute for Agricultural Research (2012)

- Autumn school Scales and patterns in the earth system, Max-Planck Institute for the Physics of Complex Systems (2012)

\section{Didactic Skills Training}

- Co-supervising MSc student with thesis entitled 'Combining chemistry and dynamics to reconstruct the ozone budget above tall vegetation' (2015-2016)

\section{Oral Presentations}

- Influence of canopy phenology changes on turbulence above tall deciduous canopy. European Geoscience Union (EGU) Conference, 12-16 April 2015, Vienna, Austria

- The role of small canopy-scale turbulence and large meso-scale forcing on convective boundary-layer dynamics. Buys-Ballot Research School for Fundamental Processes in the Climate System (BBOS) Symposium, 28-30 October 2015, Renesse, The Netherlands

- Integrating canopy and large-scale atmospheric effects in the convective boundary-layer dynamics and chemistry during CHATS experiment. 32nd Conference on Agricultural and Forest Meteorology, 22nd Symposium on Boundary Layers and Turbulence, and Third Conference on Biogeosciences, 20-24 June 2016, Salt-Lake City, United States of America

SENSE Coordinator PhD Education

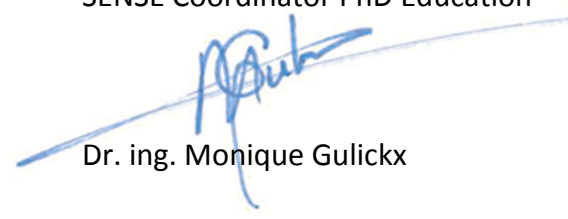


This project was financially supported by the Max Planck Institute for Chemistry, Mainz, Germany

This study was supported by the CHATS community by providing excess to the high quality observational data set.

Financial support from Wageningen University for printing this thesis is gratefully acknowledged.

Printed by Digiforce 